\title{
Pd-Catalyzed Double Decarbonylative Aryl Sulfide Synthesis through Aryl Exchange between Amides and Thioesters
}

\author{
Fusheng Bie,${ }^{\dagger}$ Xuejing Liu, ${ }^{\dagger}$ Han Cao, ${ }^{\dagger}$ Yijun Shi ${ }^{\dagger}$ Tongliang Zhou, ${ }^{\ddagger}$ Michal Szostak, ${ }^{*}{ }^{\dagger}$ and \\ Chengwei Liu*,§ \\ ${ }^{\dagger}$ Shandong Lunan Coal Chemical Research Institute of Engineering and Technology, Zaozhuang \\ University, 1 Bei'an Road, Zaozhuang, Shandong 277160, China \\ ${ }^{\ddagger}$ Department of Chemistry, Rutgers University, 73 Warren Street, Newark, New Jersey 07102, \\ United States \\ ${ }^{\S}$ School of Chemistry and Materials Science, Nanjing University of Information Science and \\ Technology, 219 Ningliu Road, Nanjing, Jiangsu 210044, China
}

michal.szostak@rutgers.edu; chengwei.liu@nuist.edu.cn

\section{Supporting Information}

\section{Table of Contents}

General Information

Experimental Procedures

Characterization Data for Amides

Characterization Data for Thioesters

Aryl Sulfide Synthesis between Amides and Thioesters

Mechanistic Studies

References

${ }^{1} \mathrm{H},{ }^{13} \mathrm{C}$ and ${ }^{19} \mathrm{~F}$ NMR Spectra of Amides

${ }^{1} \mathrm{H},{ }^{13} \mathrm{C}$ and ${ }^{19} \mathrm{~F}$ NMR Spectra of Thioesters

70

${ }^{1} \mathrm{H},{ }^{13} \mathrm{C}$ and ${ }^{19} \mathrm{~F}$ NMR Spectra of Thioethers

\section{Corresponding Author:}

Prof. Dr. M. Szostak

Rutgers University michal.szostak@rutgers.edu
Prof. Dr. C. Liu

Nanjing University of Information Science and Technology chengwei.liu@nuist.edu.cn 


\section{General Information}

All starting materials reported in the manuscript have been prepared according to the method

reported previously. ${ }^{1-10}$ Spectroscopic data matched literature values. All experiments involving palladium were performed using standard Schlenk techniques under argon atmosphere unless stated otherwise. All solvents were purchased at the highest commercial grade and used as received or after purification by passing through activated alumina columns or distillation from sodium/benzophenone under nitrogen. All solvents were deoxygenated prior to use. All the other chemicals were purchased at the highest commercial grade and used as received. Reaction glassware was oven-dried at $140{ }^{\circ} \mathrm{C}$ for at least $24 \mathrm{~h}$ or flame-dried prior to use, allowed to cool under vacuum and purged with argon (three cycles). All products were identified using ${ }^{1} \mathrm{H}$ NMR analysis and comparison with authentic samples. GC and/or GC/MS analysis was used for volatile products. All yields refer to yields determined by ${ }^{1} \mathrm{H}$ NMR and/or GC or GC/MS using an internal standard (optimization) and isolated yields (preparative runs) unless stated otherwise. ${ }^{1} \mathrm{H} N M R,{ }^{13} \mathrm{C}$ NMR and ${ }^{19} \mathrm{~F}$ NMR spectra were recorded in $\mathrm{CDCl}_{3}$ on Bruker spectrometers at $400 \mathrm{MHz}\left({ }^{1} \mathrm{H}\right.$ NMR), $100 \mathrm{MHz}\left({ }^{13} \mathrm{C} \mathrm{NMR}\right)$ and $376 \mathrm{MHz}\left({ }^{19} \mathrm{~F} \mathrm{NMR}\right)$. All shifts are reported in parts per million (ppm) relative to residual $\mathrm{CHCl}_{3}$ peak (7.26 and $77.16 \mathrm{ppm},{ }^{1} \mathrm{H}$ NMR and ${ }^{13} \mathrm{C}$ NMR, respectively). All coupling constants $(J)$ are reported in hertz $(\mathrm{Hz})$. Abbreviations are: s, singlet; d, doublet; t, triplet; q, quartet; brs, broad singlet. GC-MS chromatography was performed using Agilent 7890A GC System and Agilent 7000B inert XL EI MSD using helium as the carrier gas at a flow rate of 1 $\mathrm{mL} / \mathrm{min}$ and an initial oven temperature of $50{ }^{\circ} \mathrm{C}$. The injector temperature was $250{ }^{\circ} \mathrm{C}$. The detector temperature was $250{ }^{\circ} \mathrm{C}$. For runs with the initial oven temperature of $50^{\circ} \mathrm{C}$, temperature was increased with a $10{ }^{\circ} \mathrm{C} / \mathrm{min}$ ramp after $50^{\circ} \mathrm{C}$ hold for $3 \mathrm{~min}$ to a final temperature of $220^{\circ} \mathrm{C}$, then hold at $220^{\circ} \mathrm{C}$ for $15 \mathrm{~min}$ (splitless mode of injection, total run time of $22.0 \mathrm{~min}$ ). HRMS data were recorded on Agilent 6530 Accurate-Mass Q-TOF LCMS spectrometer by ESI in positive mode. Melting point was recorded on INESA (SGWX-4B) equipment without corrected. All flash chromatography was performed using silica gel, 300 mesh. TLC analysis was carried out on glass plates coated with silica gel 60 F254, $0.2 \mathrm{~mm}$ thickness. The plates were visualized using a $254 \mathrm{~nm}$ ultraviolet lamp or aqueous potassium permanganate solutions. ${ }^{1} \mathrm{H}$ NMR and ${ }^{13} \mathrm{C}$ NMR data are given for all compounds in the Supporting Information. ${ }^{1} \mathrm{H}$ NMR, ${ }^{13} \mathrm{C}$ NMR and HRMS data are reported for all new compounds. 


\section{Experimental Procedures}

General Procedure for Activated Tertiary Amide Synthesis. A previously published procedure was followed. ${ }^{1-7}$ An oven-dried vial $(20 \mathrm{~mL})$ equipped with a stir bar was charged with amine (5.0 mmol, 1.0 equiv), dimethylaminopyridine (typically, 0.005 equiv), triethylamine (typically, 1.2 equiv), and dichloromethane (typically, $10 \mathrm{~mL}$ ), placed under a positive pressure of argon, and subjected to three evacuation/backfilling cycles under high vacuum. Acyl chloride (typically, 1.0 equiv) was added dropwise to the reaction mixture with vigorous stirring at $0{ }^{\circ} \mathrm{C}$, and the reaction mixture was stirred $12 \mathrm{~h}$ at room temperature. After the indicated time, the reaction mixture was diluted with ethyl acetate $(30 \mathrm{~mL})$, washed with $1 \mathrm{M} \mathrm{HCl}(20 \mathrm{~mL}), \mathrm{H}_{2} \mathrm{O}$ $(20 \mathrm{~mL})$, brine $(20 \mathrm{~mL})$. Then the organic layer was dried by $\mathrm{Na}_{2} \mathrm{SO}_{4}$, filtrated and concentrated. Unless stated otherwise, the crude product was purified by recrystallization (toluene) to give analytically pure product.

General Procedure for Activated Secondary Amide Synthesis. An oven-dried flask (25 mL) equipped with a stir bar was charged with aniline (typically, $5.0 \mathrm{mmol}, 1.0$ equiv), pyridine (typically, 2.5 equiv) and dichloromethane (typically, $0.50 \mathrm{M}$ ), placed under a positive pressure of argon, and subjected to three evacuation/backfilling cycles under high vacuum. $p$ Methylbenzenesulfonyl chloride (typically, 1.0 equiv) was added portions to the reaction mixture with vigorous stirring at $0{ }^{\circ} \mathrm{C}$, and the reaction mixture was stirred for $2 \mathrm{~h}$ at room temperature. After the indicated time, the reaction mixture was diluted with ethyl acetate $(30 \mathrm{~mL})$. The reaction mixture was washed with $\mathrm{HCl}(10 \mathrm{~mL}), \mathrm{H}_{2} \mathrm{O}(10 \mathrm{~mL})$, brine $(10 \mathrm{~mL})$, dried, and concentrated to get crude first-step product. Then an oven-dried flask $(25 \mathrm{~mL})$ equipped with a stir bar was charged with crude first-step product (typically, 5.0 mmol, 1.0 equiv), 4(dimethylamino)pyridine (typically, 0.005 equiv), triethylamine (typically, 1.2 equiv) and dichloromethane (typically, $0.50 \mathrm{M}$ ), placed under a positive pressure of argon, and subjected to three evacuation/backfilling cycles under high vacuum. Acyl chloride (typically, 1.0 equiv) was added dropwise to the reaction mixture with vigorous stirring at $0{ }^{\circ} \mathrm{C}$, and the reaction mixture was stirred for $12 \mathrm{~h}$ at room temperature. After the indicated time, the reaction mixture was diluted with ethyl acetate $(30 \mathrm{~mL})$. The reaction mixture was washed with $\mathrm{HCl}(10 \mathrm{~mL})$, brine $(10 \mathrm{~mL}), \mathrm{H}_{2} \mathrm{O}(10 \mathrm{~mL})$, dried, and concentrated. The crude product was purified by recrystallization (toluene) to give analytically pure product. 
General Procedure for Activated Primary Amide Synthesis. A previously published procedure was followed. An oven-dried vial $(20 \mathrm{~mL})$ equipped with a stir bar was charged with benzamide (5.0 mmol, 1.0 equiv), dimethylaminopyridine (typically, 0.1 equiv), and dichloromethane (typically, $10 \mathrm{~mL}$ ), placed under a positive pressure of argon, and subjected to three evacuation/backfilling cycles under high vacuum. Di-tert-butyl dicarbonate (typically, 2.0 equiv) was added portions to the reaction mixture with vigorous stirring at $0{ }^{\circ} \mathrm{C}$, and the reaction mixture was stirred $12 \mathrm{~h}$ at room temperature. After the indicated time, the reaction mixture was diluted with ethyl acetate $(30 \mathrm{~mL})$, washed with $1 \mathrm{M} \mathrm{HCl}(20 \mathrm{~mL}), \mathrm{H}_{2} \mathrm{O}(20 \mathrm{~mL})$, brine $(20 \mathrm{~mL})$. Then the organic layer was dried, filtrated and concentrated. The crude product was purified by column chromatography (ethyl acetate/hexane) to give pure product.

General Procedure for Thioester Synthesis. An oven-dried flask (25 mL) equipped with a stir bar was charged with thiophenol (typically, $5.0 \mathrm{mmol}, 1.0$ equiv), acyl chloride (typically, 1.0 equiv), and dichloromethane (typically, $0.50 \mathrm{M}$ ), placed under a positive pressure of argon, and subjected to three evacuation/backfilling cycles under high vacuum. Triethylamine (typically, 2.0 equiv) was added dropwise to the reaction mixture with vigorous stirring at $0{ }^{\circ} \mathrm{C}$, and the reaction mixture was stirred for $12 \mathrm{~h}$ at room temperature. After the indicated time, the reaction mixture was diluted with ethyl acetate $(30 \mathrm{~mL})$. The reaction mixture was washed with $\mathrm{HCl}(1 \mathrm{x}$ $10 \mathrm{~mL})$, brine $(1 \times 10 \mathrm{~mL}), \mathrm{H}_{2} \mathrm{O}(1 \times 10 \mathrm{~mL})$, dried, and concentrated. The crude product was washed with hexane to give analytically pure product.

General Procedure for Aryl Sulfide Synthesis between Amides and Thioesters. An ovendried vial equipped with a stir bar was charged with amide substrate (neat, 1.0 equiv) and thioester substrate (neat, 1.0 equiv), $\mathrm{Pd}(\mathrm{OAc})_{2}$ (typically, $3 \mathrm{~mol} \%$ ) and 4,5bis(diphenylphosphino)-9,9-dimethylxanthene (XantPhos, typically, 6 mol\%), placed under a positive pressure of argon, and subjected to three evacuation/backfilling cycles under high vacuum. Toluene (typically, $0.20 \mathrm{M}$ ) was added with vigorous stirring at room temperature, the reaction mixture was placed in a preheated oil bath at $160{ }^{\circ} \mathrm{C}$, and stirred for $15 \mathrm{~h}$ at $160{ }^{\circ} \mathrm{C}$. After the indicated time, the reaction mixture was cooled down to room temperature. A sample was analyzed by ${ }^{1} \mathrm{H} \mathrm{NMR}\left(\mathrm{CDCl}_{3}, 400 \mathrm{MHz}\right)$ and gas chromatography-mass spectrometry (GC-MS) to obtain yield using internal standard and comparison with authentic samples. Purification by 
chromatography on silica gel (hexane/dichloromethane) afforded the title products. Note: selectivity for aryl sulfide synthesis is $>90: 10$ in all cases examined. The selectivity refers to the ratio of aryl exchange product vs. direct decarbonylation product.

\section{Representative Procedure for Aryl Sulfide Synthesis between Amides and Thioesters. An} oven-dried vial equipped with a stir bar was charged with 1-(4(trifluoromethyl)benzoyl)piperidine-2,6-dione $(28.6 \mathrm{mg}, 0.10 \mathrm{mmol}), S$-phenyl benzothioate (21.5 mg, $0.1 \mathrm{mmol}, 1.0$ equiv), $\mathrm{Pd}(\mathrm{OAc})_{2}(0.7 \mathrm{mg}, 0.003 \mathrm{mmol}, 0.03$ equiv) and XantPhos (3.5 $\mathrm{mg}, 0.006 \mathrm{mmol}, 0.06$ equiv), placed under a positive pressure of argon, and subjected to three evacuation/backfilling cycles under high vacuum. Toluene $(0.5 \mathrm{~mL}, 0.20 \mathrm{M})$ was added with vigorous stirring at room temperature, the reaction mixture was placed in a preheated oil bath at $160^{\circ} \mathrm{C}$, and stirred for $15 \mathrm{~h}$ at $160^{\circ} \mathrm{C}$. After the indicated time, the reaction mixture was cooled down to room temperature. Purification by chromatography on silica gel (hexane/DCM $=25 / 1$ ) afforded (phenyl(4-(trifluoromethyl)phenyl)sulfane (3a). Yield 92\% (23.4 mg, $0.092 \mathrm{mmol})$. White solid. Characterization data are included in the section below.

\section{Representative Example for Aryl Sulfide Synthesis between Amides and Thioesters (1.0 mmol scale)}

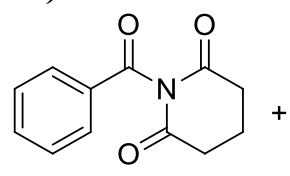

$1 \mathrm{c}$

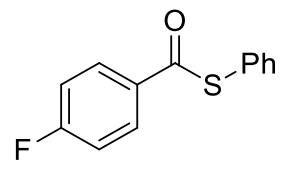

2c

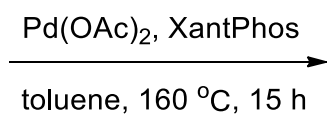

toluene, $160{ }^{\circ} \mathrm{C}, 15 \mathrm{~h}$

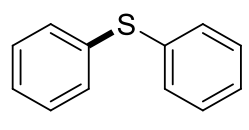

$3 \mathbf{i}$

An oven-dried vial equipped with a stir bar was charged with 1-benzoylpiperidine-2,6-dione (217.2 $\mathrm{mg}, 1.0 \mathrm{mmol}), S$-phenyl 4-fluorobenzothioate $(232.3 \mathrm{mg}, 1.0 \mathrm{mmol}, 1.0$ equiv), $\mathrm{Pd}(\mathrm{OAc})_{2}(6.8 \mathrm{mg}, 0.03 \mathrm{mmol}, 0.03$ equiv) and XantPhos (34.7 mg, $0.06 \mathrm{mmol}, 0.06$ equiv), placed under a positive pressure of argon, and subjected to three evacuation/backfilling cycles under high vacuum. Toluene $(5 \mathrm{~mL}, 0.20 \mathrm{M})$ was added with vigorous stirring at room temperature, the reaction mixture was placed in a preheated oil bath at $160^{\circ} \mathrm{C}$, and stirred for 15 $\mathrm{h}$ at $160^{\circ} \mathrm{C}$. After the indicated time, the reaction mixture was cooled down to room temperature. Purification by chromatography on silica gel (hexane) afforded diphenylsulfane (3i). Yield 90\% $(167.5 \mathrm{mg}, 0.90 \mathrm{mmol})$. White solid. Characterization data are included in the section below. 


\section{Characterization Data for Amides}

Note: All starting materials have been prepared according to the previously published procedure. ${ }^{1-7}$ The yields have not been optimized.

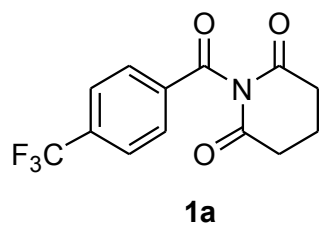

1-(4-(Trifluoromethyl)benzoyl)piperidine-2,6-dione (1a). ${ }^{1}$ Yield: $72 \%$ (1.026 g). White solid. ${ }^{1}$ H NMR (400 MHz, $\left.\mathbf{C D C l}_{3}\right) \delta$ 7.98-7.96 (d, $\left.J=8.0 \mathrm{~Hz}, 2 \mathrm{H}\right), 7.76-7.74(\mathrm{~d}, J=8.0 \mathrm{~Hz}, 2 \mathrm{H})$, 2.81-2.78 (t, $J=8.0 \mathrm{~Hz}, 4 \mathrm{H}), 2.20-2.13(\mathrm{~m}, 2 \mathrm{H}) .{ }^{13} \mathbf{C}$ NMR (100 MHz, $\left.\mathbf{C D C l}_{3}\right) \delta 172.1,170.2$, $136.3\left(\mathrm{q}, J^{2}=33 \mathrm{~Hz}\right), 134.9,130.5,126.4\left(\mathrm{q}, J^{3}=3.0 \mathrm{~Hz}\right), 124.7\left(\mathrm{~d}, J^{l}=271 \mathrm{~Hz}\right), 32.5$, 17.6. ${ }^{19} \mathbf{F}$ $\left(376 \mathbf{M H z}, \mathbf{C D C l}_{3}\right) \delta-63.38$.

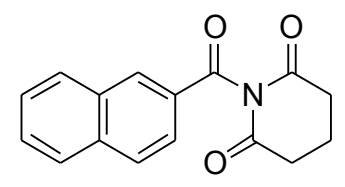

$1 \mathrm{~b}$

1-(2-Naphthoyl)piperidine-2,6-dione (1b). ${ }^{2}$ Yield: 92\% (1.240 g). White solid. ${ }^{1}$ H NMR (400 $\underline{\left.\mathbf{M H z}, \mathbf{C D C l}_{3}\right)} \delta 8.34(\mathrm{~s}, 1 \mathrm{H}), 7.95-7.87(\mathrm{~m}, 3 \mathrm{H}), 7.89-7.87(\mathrm{~d}, J=8.3 \mathrm{~Hz}, 2 \mathrm{H}), 7.66-7.62(\mathrm{~m}, 1$ H), 7.58-7.54 (m, $1 \mathrm{H}), 2.84-2.81(\mathrm{t}, J=6.5 \mathrm{~Hz}, 4 \mathrm{H}), 2.23-2.16(\mathrm{~m}, 2 \mathrm{H}) .{ }^{13} \mathbf{C} \mathbf{N M R}(\mathbf{1 0 0} \mathbf{~ M H z}$, $\left.\mathbf{C D C l}_{3}\right) \delta 172.2,171.0,136.6,132.8,132.6,130.0,129.6,129.3,129.3,128.0,127.3,124.9,32.6$, 17.7.

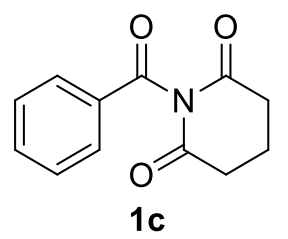

1-Benzoylpiperidine-2,6-dione (1b). ${ }^{1}$ Yield: $80 \%$ (0.867 g). White solid. ${ }^{1}$ H NMR (400 MHz, $\left.\mathbf{C D C l}_{\underline{3}}\right) \delta 7.88-7.86(\mathrm{~d}, J=8.0 \mathrm{~Hz}, 2 \mathrm{H}), 7.66-7.62(\mathrm{t}, J=8.0 \mathrm{~Hz}, 1 \mathrm{H}), 7.51-7.47$ (t, $J=8.0 \mathrm{~Hz}$, $2 \mathrm{H}), 2.80-2.76(\mathrm{t}, J=8.0 \mathrm{~Hz}, 4 \mathrm{H}), 2.19-2.12(\mathrm{~m}, 2 \mathrm{H}) .{ }^{13} \mathbf{C} \mathbf{N M R}\left(\mathbf{1 0 0} \mathbf{M H z}, \mathbf{C D C l}_{3}\right) \delta 172.0$, $170.9,135.1,131.9,130.3,129.3,32.5,17.6$. 


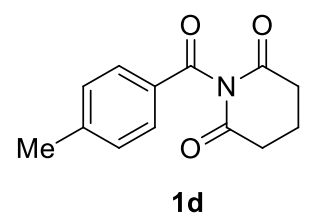

1-(4-Methylbenzoyl)piperidine-2,6-dione (1d). ${ }^{1}$ Yield: 70\% (0.813 g). Light brown solid. ${ }^{1} \mathbf{H}$ NMR (400 MHz, $\left.\mathbf{C D C l}_{3}\right) \delta$ 7.76-7.74 (d, $\left.J=8.0 \mathrm{~Hz}, 2 \mathrm{H}\right), 7.29-7.27(\mathrm{~d}, J=8.0 \mathrm{~Hz}, 2 \mathrm{H}), 2.78$ $2.75(\mathrm{t}, J=8.0 \mathrm{~Hz}, 4 \mathrm{H}), 2.42(\mathrm{~s}, 3 \mathrm{H}), 2.17-2.10$ (m, $2 \mathrm{H}) .{ }^{13} \mathbf{C}$ NMR (100 MHz, $\left.\mathbf{C D C l}_{3}\right) \delta 172.0$, $170.5,146.5,130.4,130.0,129.3,32.5,22.0,17.6$.

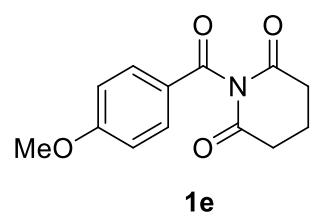

1-(4-Methoxybenzoyl)piperidine-2,6-dione (1e). ${ }^{1}$ Yield: 92\% (1.131 g). Brown solid. ${ }^{1}$ H NMR $\left(400 \mathrm{MHz}, \mathbf{C D C l}_{3}\right) \delta$ 7.83-7.81 (d, $\left.J=9.0 \mathrm{~Hz}, 2 \mathrm{H}\right), 6.95-6.93(\mathrm{~d}, J=9.0 \mathrm{~Hz}, 2 \mathrm{H}), 3.86(\mathrm{~s}, 3 \mathrm{H})$, 2.76-2.73 (t, $J=6.5 \mathrm{~Hz}, 4 \mathrm{H}), 2.14-2.08(\mathrm{~m}, 2 \mathrm{H}) .{ }^{13} \mathbf{C}$ NMR (100 MHz, CDCl 3 ) $\delta 172.1169 .7$, $165.2,132.9,124.5,114.6,55.8,32.5,17.6$.

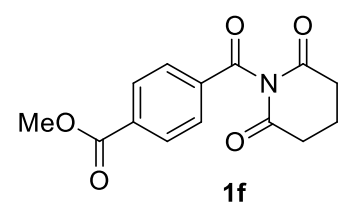

Methyl 4-(2,6-dioxopiperidine-1-carbonyl)benzoate (1f). ${ }^{1}$ Yield: $83 \%$ (1.140 g). White solid. ${ }^{1}$ H NMR (400 MHz, CDCl $\left._{3}\right) \delta 8.15-8.12(\mathrm{~m}, 2 \mathrm{H}), 7.92-7.90(\mathrm{~m}, 2 \mathrm{H}), 3.95(\mathrm{~s}, 3 \mathrm{H}), 2.81-2.76$ $(\mathrm{t}, J=8.0 \mathrm{~Hz}, 4 \mathrm{H}), 2.20-2.13$ (m, $2 \mathrm{H}) .{ }^{13} \mathbf{C}$ NMR (100 $\left.\mathbf{~ M H z} \mathbf{C D C l}_{3}\right) \delta 172.1,170.5,165.9$, $135.5,135.3,130.4,130.1,52.8,32.5,17.6$.

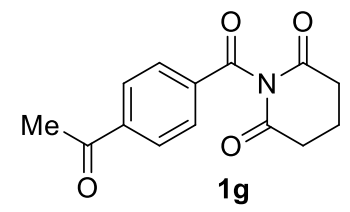

1-(4-Acetylbenzoyl)piperidine-2,6-dione (1g). ${ }^{1}$ Yield: $53 \%$ (0.693 g). Brown solid. ${ }^{1}$ H NMR (400 MHz, $\left.\mathbf{C D C l}_{3}\right) \delta$ 8.05-8.02 (m, $\left.2 \mathrm{H}\right), 7.95-7.93$ (m, $\left.2 \mathrm{H}\right), 2.80-2.77$ (t, $\left.J=4.0 \mathrm{~Hz}, 4 \mathrm{H}\right), 2.64$ 
(s, $3 \mathrm{H}), 2.19-2.13$ (m, $2 \mathrm{H}) \cdot{ }^{13} \mathbf{C}$ NMR (100 MHz, $\left.\mathbf{C D C l}_{3}\right) \delta 197.2,172.1,170.5,141.5,135.2$, 130.4, 129.0, 32.5, 27.1, 17.6.

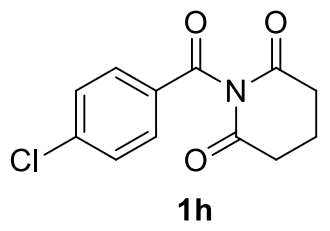

1-(4-Chlorobenzoyl)piperidine-2,6-dione (1h). ${ }^{1}$ Yield: 72\% (0.906 g). White solid. ${ }^{1}$ H NMR $\left(400 \mathrm{MHz}, \mathbf{C D C l}_{3}\right) \delta$ 7.80-7.78 (d, $\left.J=8.6 \mathrm{~Hz}, 2 \mathrm{H}\right), 7.47-7.45(\mathrm{~d}, J=8.6 \mathrm{~Hz}, 2 \mathrm{H}), 2.79-2.77(\mathrm{t}$, $J=6.5 \mathrm{~Hz}, 4 \mathrm{H}), 2.18-2.11$ (m, $2 \mathrm{H}) .{ }^{13} \mathbf{C}$ NMR (100 MHz, $\left.\mathbf{C D C l}_{3}\right) \delta 172.0,170.0,141.8,131.6$, $130.4,129.7,32.5,17.6$.

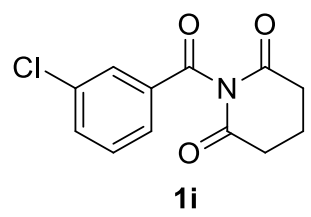

1-(3-Chlorobenzoyl)piperidine-2,6-dione (1i). ${ }^{1}$ Yield: 77\% (0.966 g). White solid. ${ }^{1}$ H NMR $\left(400 \mathrm{MHz}, \mathbf{C D C l}_{3}\right) \delta 7.81(\mathrm{~s}, 1 \mathrm{H}), 7.75-7.72(\mathrm{~m}, 1 \mathrm{H}), 7.62-7.59(\mathrm{~m}, 1 \mathrm{H}), 7.45-7.42(\mathrm{t}, J=8.2$ $\mathrm{Hz}, 1 \mathrm{H}), 2.80-2.76(\mathrm{t}, J=6.5 \mathrm{~Hz}, 4 \mathrm{H}), 2.18-2.12(\mathrm{~m}, 2 \mathrm{H}) .{ }^{13} \mathbf{C}$ NMR $\left(\mathbf{1 0 0} \mathbf{M H z} \mathbf{C D C l}_{3}\right) \delta$ 172.0, 170.0, 135.5, 135.0, 133.6, 130.6, 130.1, 128.9, 32.5, 17.6.

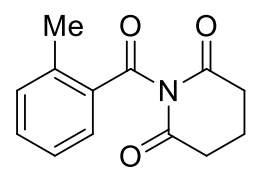

$1 \mathrm{j}$

1-(2-Methylbenzoyl)piperidine-2,6-dione (1j). ${ }^{1}$ Yield: 68\% (0.788 g). Light brown solid. ${ }^{1} \mathbf{H}$ NMR (400 MHz, CDCl $\underline{3}) \delta$ 7.50-7.48 (d, $J=7.6 \mathrm{~Hz}, 1 \mathrm{H}), 7.47-7.45(\mathrm{t}, J=7.6 \mathrm{~Hz}, 1 \mathrm{H}), 7.33-$ $7.31(\mathrm{~d}, J=7.6 \mathrm{~Hz}, 1 \mathrm{H}), 7.27-7.23(\mathrm{t}, J=7.6 \mathrm{~Hz}, 1 \mathrm{H}), 2.77-2.74$ (t, $J=6.6 \mathrm{~Hz}, 4 \mathrm{H}), 2.68$ (s, 3 H), 2.16-2.09 (m, $2 \mathrm{H}) .{ }^{13} \mathbf{C}$ NMR (100 MHz, $\left.\mathbf{C D C l}_{3}\right) \delta 172.2,170.8,142.6,133.9,132.5,131.2$, $130.8,126.3,32.5,22.0,17.5$. 


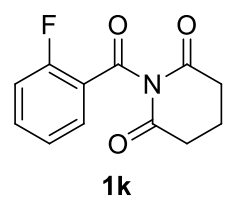

1-(2-Fluorobenzoyl)piperidine-2,6-dione (1k). ${ }^{1}$ Yield: 83\% (0.979 g). White solid. ${ }^{1} \mathbf{H}$ NMR $\left(400 \mathrm{MHz}, \mathbf{C D C l}_{3}\right) \delta 8.10-8.06(\mathrm{~m}, 1 \mathrm{H}), 7.64-7.58(\mathrm{~m}, 1 \mathrm{H}), 7.32-7.28(\mathrm{~m}, 1 \mathrm{H}), 7.13-7.08(\mathrm{~m}$, $1 \mathrm{H}), 2.75-2.72(\mathrm{t}, J=6.5 \mathrm{~Hz}, 4 \mathrm{H}), 2.14-2.07(\mathrm{~m}, 2 \mathrm{H}) .{ }^{13} \mathbf{C}$ NMR $\left(\mathbf{1 0 0} \mathbf{~ M H z}, \mathbf{C D C l}_{3}\right) \delta 171.8$, $167.0\left(\mathrm{~d}, J^{5}=1.5 \mathrm{~Hz}\right), 163.3\left(\mathrm{~d}, J^{l}=256.1 \mathrm{~Hz}\right), 137.0\left(\mathrm{~d}, J^{3}=10.0 \mathrm{~Hz}\right), 133.1,125.3\left(\mathrm{~d}, J^{F}=3.5\right.$ $\mathrm{Hz}), 120.5\left(\mathrm{~d}, J^{4}=8.0 \mathrm{~Hz}\right), 117.3\left(\mathrm{~d}, J^{2}=23.6 \mathrm{~Hz}\right), 32.5,17.4 .{ }^{19} \mathbf{F} \mathbf{N M R}\left(376 \mathbf{~ M H z}, \mathbf{C D C l}_{3}\right) \delta$ 113.52 .

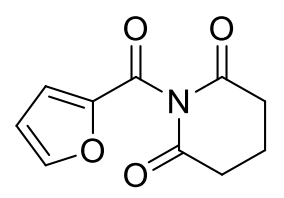

11

1-(Furan-2-carbonyl)piperidine-2,6-dione (11). ${ }^{3}$ Yield: 75\% (0.783 g). Light brown solid. ${ }^{1} \mathbf{H}$ NMR (400 MHz, $\left.\mathbf{C D C l}_{3}\right) \delta 7.60(\mathrm{~s}, 1 \mathrm{H}), 7.40-7.39(\mathrm{~d}, J=3.7 \mathrm{~Hz}, 1 \mathrm{H}), 6.61-6.60(\mathrm{dd}, J=3.7$, $1.7 \mathrm{~Hz}, 1 \mathrm{H}), 2.77-2.74(\mathrm{t}, J=6.5 \mathrm{~Hz}, 4 \mathrm{H}), 2.15-2.08(\mathrm{~m}, 2 \mathrm{H}) .{ }^{13} \mathbf{C ~ N M R}\left(\mathbf{1 0 0} \mathbf{M H z}, \mathbf{C D C l}_{3}\right) \delta$ 171.7, 159.4, 148.3, 147.6, 122.1, 113.7, 32.5, 17.5.

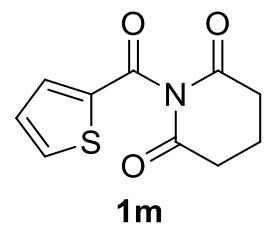

1-(Thiophene-2-carbonyl)piperidine-2,6-dione (1m). ${ }^{1}$ Yield: $74 \%(0.827 \mathrm{~g})$. White solid. ${ }^{\mathbf{1}} \mathbf{H}$ NMR (400 MHz, $\left.\mathbf{C D C l}_{3}\right) \delta$ 7.77-7.76 (dd, $\left.J=5.0,1.2,1 \mathrm{H}\right), 7.69-7.68(\mathrm{dd}, J=3.9,1.2,1 \mathrm{H})$, 7.16-7.14 (dd, $J=5.0,3.9,1 \mathrm{H}), 2.78-2.74(\mathrm{t}, J=6.5,4 \mathrm{H}), 2.15-2.08(\mathrm{~m}, 2 \mathrm{H}) .{ }^{13} \mathbf{C}$ NMR (100

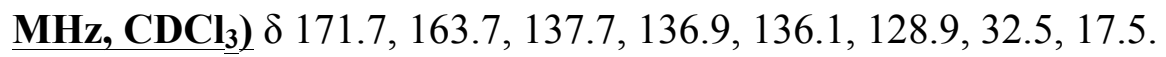




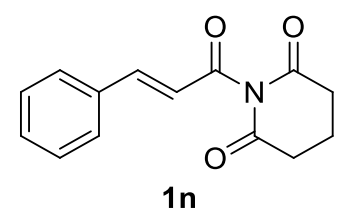

1-Cinnamoylpiperidine-2,6-dione (1n). ${ }^{4}$ Yield: $71 \%\left(0.863\right.$ g). White solid. ${ }^{1} \mathbf{H}$ NMR (400 $\left.\mathbf{M H z}, \mathbf{C D C l}_{3}\right) \delta 7.67(\mathrm{~d}, J=15.9 \mathrm{~Hz}, 1 \mathrm{H}), 7.59-7.51(\mathrm{~m}, 2 \mathrm{H}), 7.48-7.33(\mathrm{~m}, 3 \mathrm{H}), 7.30(\mathrm{~s}, 0 \mathrm{H})$, $6.68(\mathrm{~d}, J=15.9 \mathrm{~Hz}, 1 \mathrm{H}), 2.73(\mathrm{t}, J=6.5 \mathrm{~Hz}, 4 \mathrm{H}), 2.13-2.02(\mathrm{~m}, 2 \mathrm{H}) .{ }^{13} \mathbf{C}$ NMR (100 MHz, $\left.\mathbf{C D C l}_{3}\right) \delta 171.9,169.4,148.7,133.7,131.7,129.1,129.1,121.4,32.6,17.5$.

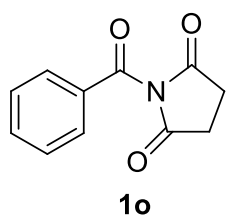

1-Benzoylpyrrolidine-2,5-dione (10). ${ }^{3}$ Yield: 89\% (0.908 g). White solid. ${ }^{1}$ H NMR (400 MHz, $\left.\mathbf{C D C l}_{3}\right) \delta 7.86-7.84(\mathrm{~d}, J=8.2 \mathrm{~Hz}, 2 \mathrm{H}), 7.69-7.65(\mathrm{t}, J=7.5 \mathrm{~Hz}, 1 \mathrm{H}), 7.52-7.48(\mathrm{t}, J=7.8 \mathrm{~Hz}$, 2H), 2.93 (s, 4H). ${ }^{13} \mathbf{C}$ NMR (100 MHz, $\left.\mathbf{C D C l}_{3}\right) \delta 174.7,167.8,135.3,131.5,130.7,129.1,29.2$.

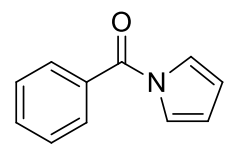

$1 p$

Phenyl(1H-pyrrol-1-yl)methanone (1p). ${ }^{5}$ Yield: 93\% (0.794 g). Brown oil. ${ }^{1}$ H NMR (400

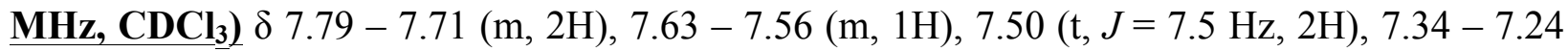
(m, 2H), $6.40-6.31(\mathrm{~m}, 2 \mathrm{H}) .{ }^{13} \mathbf{C}$ NMR (100 MHz, $\left.\mathbf{C D C l}_{3}\right) \delta 167.8,133.3,132.3,129.5,128.5$, 121.3, 113.2.

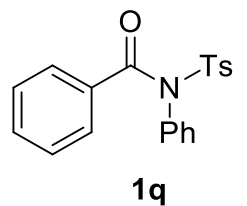

$N$-Phenyl- $N$-tosylbenzamide (1q). ${ }^{1}$ Yield: 76\% (1.344 g). White solid. ${ }^{1}$ H NMR (400 MHz, $\left.\mathbf{C D C l}_{3}\right) \delta 7.84-7.82(\mathrm{~d}, J=8.4 \mathrm{~Hz}, 2 \mathrm{H}), 7.45-7.43(\mathrm{~d}, J=7.0 \mathrm{~Hz}, 2 \mathrm{H}), 7.32-7.30(\mathrm{~d}, J=8.2 \mathrm{~Hz}$, 2H), 7.28-7.26 (m, 4H), 7.18-7.14 (m, 4H), 2.45 (s, 3H). $\left.{ }^{13} \mathbf{C ~ N M R ~ ( 1 0 0 ~ M H z , ~} \mathbf{C D C l}_{3}\right) \delta$ 170.0, $145.0,137.5,135.3,133.8,131.9,130.5,129.6,129.4,129.2,129.2,128.1,21.8$. 


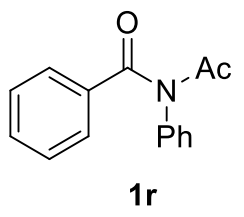

$\mathrm{N}$-Acetyl- $\mathrm{N}$-phenylbenzamide (1r). ${ }^{1}$ Yield: $86 \%$ (1.028 g). Light yellow solid. ${ }^{\mathbf{1} H}$ NMR (400 $\underline{\left.\mathbf{M H z}, \mathbf{C D C l}_{3}\right)} \delta 7.65-7.57(\mathrm{~m}, 2 \mathrm{H}), 7.44-7.37(\mathrm{~m}, 1 \mathrm{H}), 7.37-7.22(\mathrm{~m}, 5 \mathrm{H}), 7.19-7.12(\mathrm{~m}$, 2H), 2.43 (s, 3H). ${ }^{13} \mathbf{C}$ NMR (100 MHz, $\left.\mathbf{C D C l}_{3}\right) \delta$ 173.7, 173.0, 139.2, 134.9, 132.2, 129.5, 129.3, 128.7, 128.4, 128.2, 25.8 .

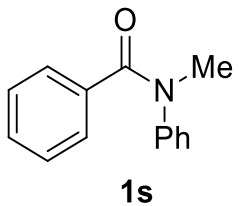

$\boldsymbol{N}$-Methyl- $\boldsymbol{N}$-phenylbenzamide (1s). ${ }^{3}$ Yield $89 \%(0.944 \mathrm{~g})$. Yellow oil. ${ }^{1} \mathrm{H}$ NMR (400 MHz, $\left.\mathbf{C D C l}_{3}\right) \delta$ 7.30-7.28 (d, $\left.J=7.0 \mathrm{~Hz}, 2 \mathrm{H}\right), 7.23-7.19(\mathrm{~m}, 3 \mathrm{H}), 7.16-7.10(\mathrm{~m}, 3 \mathrm{H}), 7.03-7.02(\mathrm{~d}, J$ $=7.8 \mathrm{~Hz}, 2 \mathrm{H}), 3.49$ (s, $3 \mathrm{H}) \cdot{ }^{13} \mathbf{C}$ NMR (100 MHz, $\left.\mathbf{C D C l}_{3}\right) \delta$ 170.7, 145.0, 136.0, 129.7, 129.2, $128.8,127.8,127.0,126.6,38.5$.

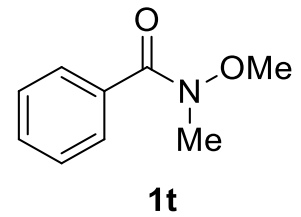

$N$-Methoxy- $N$-methylbenzamide (1t). ${ }^{6}$ Yield 85\% (0.706 g). Colorless oil. ${ }^{1}$ H NMR (400 MHz, $\left.\mathbf{C D C l}_{3}\right) \delta 7.65-7.63(\mathrm{~d}, J=6.6 \mathrm{~Hz}, 2 \mathrm{H}), 7.44-7.35(\mathrm{~m}, 3 \mathrm{H}), 3.52(\mathrm{~s}, 3 \mathrm{H}), 3.33(\mathrm{~s}, 3 \mathrm{H}) .{ }^{13} \mathbf{C}$ NMR (100 MHz, $\left.\mathbf{C D C l}_{3}\right) \delta 169.9,134.1,130.6,128.1,128.0,61.0,33.8$.

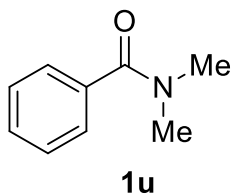

$\mathrm{N}, \mathrm{N}$-Dimethylbenzamide (1u). ${ }^{5}$ Yield 78\% (0.582 g). Colorless oil. ${ }^{1} \mathrm{H}$ NMR (400 MHz, $\left.\underline{\mathbf{C D C l}}_{3}\right) \delta$ 7.42-7.37 (m, $\left.5 \mathrm{H}\right), 3.11$ (s, $\left.3 \mathrm{H}\right), 2.97$ (s, $\left.3 \mathrm{H}\right) .{ }^{13} \mathbf{C}$ NMR (100 MHz, $\left.\mathbf{C D C l}_{3}\right) \delta 171.5$, $136.3,129.4,128.3,127.0,39.5,35.2$. 


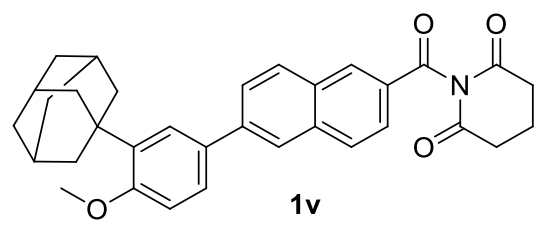

1-(6-(3-(Adamantan-1-yl)-4-methoxyphenyl)-2-naphthoyl)piperidine-2,6-dione (1v). Yield: $54 \%$ (1.37 g). New compound. Yellow solid. MP $=129-131{ }^{\circ} \mathrm{C} .{ }^{1} \mathbf{H}$ NMR (400 MHz, CDCl $\left.\underline{3}\right) \delta$ $8.34(\mathrm{~s}, 1 \mathrm{H}), 8.01(\mathrm{~s}, 1 \mathrm{H}), 7.99-7.89(\mathrm{~m}, 3 \mathrm{H}), 7.82(\mathrm{dd}, J=8.6,1.8 \mathrm{~Hz}, 1 \mathrm{H}), 7.61(\mathrm{~d}, J=2.4 \mathrm{~Hz}$, 1H), $7.55(\mathrm{dd}, J=8.4,2.3 \mathrm{~Hz}, 1 \mathrm{H}), 7.00$ (d, $J=8.4 \mathrm{~Hz}, 1 \mathrm{H}), 3.91$ (s, 3H), 2.84 (t, $J=6.5 \mathrm{~Hz}$, 4H), $2.22(\mathrm{~m}, 2 \mathrm{H}), 2.19(\mathrm{~d}, J=2.8 \mathrm{~Hz}, 6 \mathrm{H}), 2.11(\mathrm{~s}, 3 \mathrm{H}), 1.81(\mathrm{~s}, 6 \mathrm{H}) .{ }^{13} \mathbf{C}$ NMR (100 MHz, $\left.\mathbf{C D C l}_{3}\right) \delta 172.2,170.9,159.3,142.8,139.2,137.0,132.6,132.2,131.3,130.3,129.3,128.7$, $127.0,126.1,126.0,125.3,124.9,112.2,55.3,40.7,37.3,37.2,32.6,29.2,17.7$. HRMS (ESI) $\mathrm{m} / \mathrm{z}$ calcd for $\mathrm{C}_{33} \mathrm{H}_{33} \mathrm{NO}_{4} \mathrm{Na}[\mathrm{M}+\mathrm{Na}]^{+}$530.2302, found 530.2300.

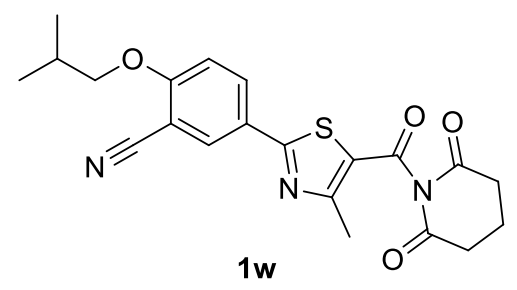

5-(5-(2,6-Dioxopiperidine-1-carbonyl)-4-methylthiazol-2-yl)-2-isobutoxybenzonitrile (1w). ${ }^{7}$ Yield: 63\% (1.296 g). White solid. ${ }^{1}$ H NMR (400 MHz, CDCl $\left.\mathbf{M}_{3}\right) \delta 8.14(\mathrm{~d}, J=2.3 \mathrm{~Hz}, 1 \mathrm{H}), 8.11$ $(\mathrm{m}, 1 \mathrm{H}), 7.01(\mathrm{~d}, J=8.8 \mathrm{~Hz}, 1 \mathrm{H}), 3.91(\mathrm{~d}, J=6.5 \mathrm{~Hz}, 2 \mathrm{H}), 2.81(\mathrm{~s}, 3 \mathrm{H}), 2.77(\mathrm{t}, J=6.5 \mathrm{~Hz}, 4 \mathrm{H})$, $2.26-2.18(\mathrm{~m}, 1 \mathrm{H}), 2.12(\mathrm{~m}, 2 \mathrm{H}), 1.09(\mathrm{~d}, J=6.7 \mathrm{~Hz}, 6 \mathrm{H}) .{ }^{13} \mathbf{C}$ NMR (100 MHz, CDCl $\left.{ }_{3}\right) \delta$ 171.6, 169.3, 165.1, 163.1, 162.8, 133.0, 132.6, 126.4, 125.4, 115.3, 112.8, 103.2, 75.9, 32.5, $28.2,19.1,18.4,17.4$.

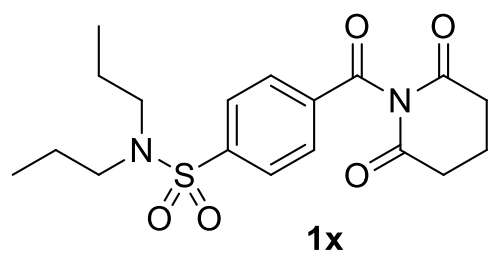

4-(2,6-Dioxopiperidine-1-carbonyl)- $N, \boldsymbol{N}$-dipropylbenzenesulfonamide $\quad(\mathbf{1 x}){ }^{4} \quad$ Yield $\quad 94 \%$ (1.782 g). White solid. ${ }^{1}$ H NMR (400 MHz, $\left.\mathbf{C D C l}_{3}\right) \delta$ 7.97-7.95 (d, $\left.J=8.6 \mathrm{~Hz}, 2 \mathrm{H}\right), 7.91-7.89$ $(\mathrm{d}, J=8.6 \mathrm{~Hz}, 2 \mathrm{H}), 3.19-3.05(\mathrm{t}, J=7.6 \mathrm{~Hz}, 4 \mathrm{H}), 2.81-2.77(\mathrm{t}, J=6.5 \mathrm{~Hz}, 4 \mathrm{H}), 2.19-2.13(\mathrm{~m}, 2$ 
H), 1.60-1.51 (m, $4 \mathrm{H}), 0.89-0.85$ (t, $J=7.4 \mathrm{~Hz}, 6 \mathrm{H}) .{ }^{13} \mathbf{C}$ NMR (100 MHz, CDCl $\left.\underline{3}\right) \delta 172.1$, $170.2,145.9,134.8,130.8,50.3,32.5,22.2,17.5,11.3$.

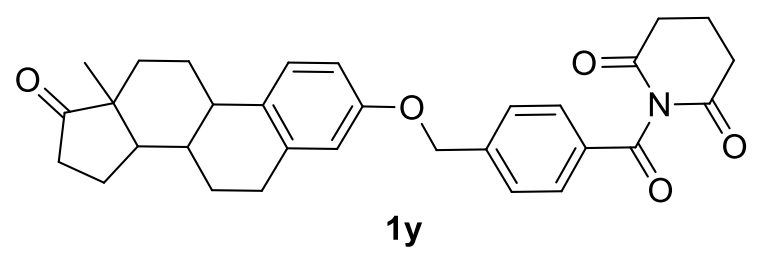

1-(4-(((13-Methyl-17-oxo-7,8,9,11,12,13,14,15,16,17-decahydro-6H-

cyclopenta[a]phenanthren-3-yl)oxy)methyl)benzoyl)piperidine-2,6-dione (1y). Yield 62\%

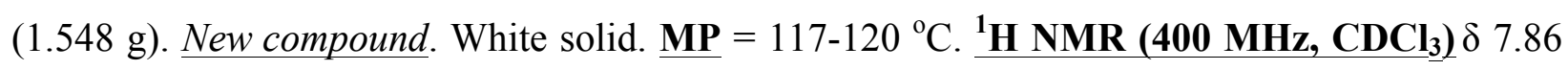
(d, $J=8.4 \mathrm{~Hz}, 2 \mathrm{H}), 7.54$ (d, $J=8.4 \mathrm{~Hz}, 2 \mathrm{H}), 7.20$ (d, $J=8.6 \mathrm{~Hz}, 1 \mathrm{H}), 6.75(\mathrm{~d}, J=8.6 \mathrm{~Hz}, 1 \mathrm{H})$, $6.68(\mathrm{~s}, 1 \mathrm{H}), 5.12(\mathrm{~s}, 2 \mathrm{H}), 2.89-2.86(\mathrm{~m}, 2 \mathrm{H}), 2.78(\mathrm{t}, J=6.5 \mathrm{~Hz}, 4 \mathrm{H}), 2.50\left(\mathrm{dd}, J_{l}=19 \mathrm{~Hz}, J_{2}\right.$ $=8.7 \mathrm{~Hz}, 1 \mathrm{H}), 2.41-2.37(\mathrm{~m}, 1 \mathrm{H}), 2.27-2.22(\mathrm{~m}, 1 \mathrm{H}), 2.18-2.12(\mathrm{~m}, 2 \mathrm{H}), 2.09-1.93(\mathrm{~m}, 3 \mathrm{H})$, $1.62-1.47$ (m, $6 \mathrm{H}), 0.90$ (s, $3 \mathrm{H}) .{ }^{13} \mathbf{C}$ NMR (100 MHz, $\left.\mathbf{C D C l}_{3}\right) \delta 172.0,170.5,156.4,145.2$, 138.1, 132.9, 131.2, 130.6, 127.5, 126.6, 115.0, 112.5, 69.1, 50.5, 48.1, 44.1, 38.4, 36.0, 32.5, 31.7, 29.8, 26.6, 26.0, 21.7, 17.6, 14.0. HRMS (ESI) $\mathrm{m} / \mathrm{z}$ calcd for $\mathrm{C}_{31} \mathrm{H}_{34} \mathrm{NO}_{5}[\mathrm{M}+\mathrm{H}]^{+}$ 500.2432 , found 500.2433 . 


\section{Characterization Data for Thioesters}

Note: All starting materials have been prepared according to the previously published procedure. ${ }^{10-14}$ The yields have not been optimized.

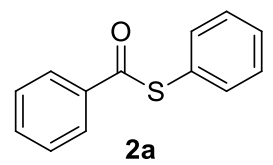

$\boldsymbol{S}$-Phenyl benzothioate (2a). ${ }^{8}$ Yield 96\% (1.029 g). White solid. ${ }^{1} \mathrm{H}$ NMR (400 MHz, $\mathbf{C D C l}_{3} \underline{)} \delta$ $8.04(\mathrm{~d}, J=7.2 \mathrm{~Hz}, 2 \mathrm{H}), 7.62(\mathrm{t}, J=7.4 \mathrm{~Hz}, 1 \mathrm{H}), 7.54-7.46(\mathrm{~m}, 7 \mathrm{H}) .{ }^{13} \mathbf{C}$ NMR (100 MHz, $\left.\mathbf{C D C l}_{3}\right) \delta 190.3,136.8,135.2,133.8,129.7,129.4,128.9,127.6,127.5$.

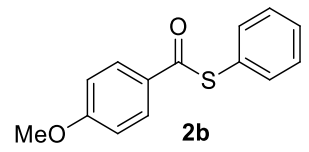

S-Phenyl 4-methoxybenzothioate (2b). ${ }^{8}$ Yield 95\% (1.158 g). White solid. ${ }^{1}$ H NMR (400 MHz, $\left.\mathbf{C D C l}_{3}\right) \delta 8.03-7.99(\mathrm{~m}, 2 \mathrm{H}), 7.53-7.50(\mathrm{~m}, 2 \mathrm{H}), 7.47-7.44(\mathrm{~m}, 3 \mathrm{H}), 6.98-6.95(\mathrm{~m}, 2 \mathrm{H})$, 3.89 (s, 3H). ${ }^{13} \mathbf{C}$ NMR (100 MHz, $\left.\mathbf{C D C l}_{3}\right) \delta 188.8,164.1,135.3,129.9,129.5,129.3,127.8$, 114.1, 55.7 .

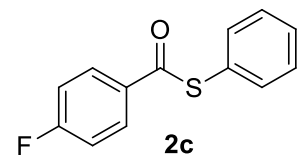

$\boldsymbol{S}$-Phenyl 4-fluorobenzothioate (2c) ${ }^{8}$ Yield 96\% (1.113 g). White solid. ${ }^{1} \mathbf{H}$ NMR (400 MHz, $\left.\mathbf{C D C l}_{3}\right) \delta 8.08$ - $8.04(\mathrm{~m}, 2 \mathrm{H}), 7.53$ - $7.46(\mathrm{~m}, 5 \mathrm{H}), 7.20$ - $7.14(\mathrm{~m}, 2 \mathrm{H}) .{ }^{\mathbf{1 3}} \mathbf{C}$ NMR (100 MHz, $\left.\mathbf{C D C l}_{3}\right) \delta 188.8,166.2\left(\mathrm{~d}, J^{l}=255.7 \mathrm{~Hz}\right), 135.2,133.1\left(\mathrm{~d}, J^{4}=2.9 \mathrm{~Hz}\right), 130.2\left(\mathrm{~d}, J^{3}=9.4 \mathrm{~Hz}\right)$, 129.8, 129.4, 127.2, $116.1\left(\mathrm{~d}, J^{2}=21.9 \mathrm{~Hz}\right) .{ }^{\mathbf{1 9}} \mathbf{F} \mathbf{N M R}\left(\mathbf{3 7 6} \mathbf{~ M H z}, \mathbf{C D C l}_{3}\right) \delta-104.13$.

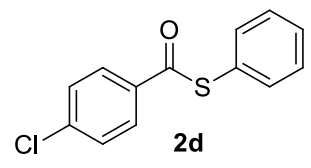

S-Phenyl 4-chlorobenzothioate (2d). ${ }^{8}$ Yield 80\% (0.990 g). White solid. ${ }^{1}$ H NMR (400 MHz, $\left.\mathbf{C D C l}_{3}\right) \delta 7.99-7.95(\mathrm{~m}, 2 \mathrm{H}), 7.53-7.49(\mathrm{~m}, 2 \mathrm{H}), 7.48-7.46(\mathrm{~m}, 5 \mathrm{H}) .{ }^{13} \mathbf{C}$ NMR (100 MHz, $\left.\mathbf{C D C l}_{3}\right) \delta 189.2,140.2,135.2,135.1,129.9,129.5,129.2,129.0,127.0$. 


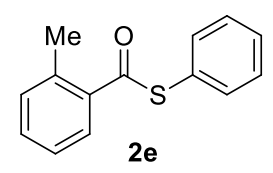

S-Phenyl 2-methylbenzothioate (2e) ${ }^{8}$ Yield 88\% (1.009 g). White solid. ${ }^{1}$ H NMR (400 MHz, $\left.\mathbf{C D C l}_{3}\right) \delta 7.96(\mathrm{~d}, J=7.6 \mathrm{~Hz}, 1 \mathrm{H}), 7.55-7.52(\mathrm{~m}, 2 \mathrm{H}), 7.50-7.42(\mathrm{~m}, 4 \mathrm{H}), 7.33-7.27(\mathrm{~m}, 2 \mathrm{H})$, 2.50 (s, 3H). ${ }^{13} \mathbf{C}$ NMR (100 MHz, $\left.\mathbf{C D C l}_{3}\right) \delta 192.3,137.6,136.9,135.0,132.2,131.9,129.6$, $129.4,128.8,128.3,126.09,20.9$.

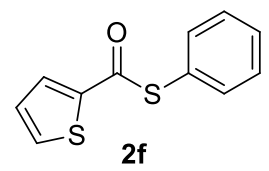

S-Phenyl thiophene-2-carbothioate (2f). ${ }^{8}$ Yield 76\% (0.835 g). Pale yellow solid. ${ }^{1} \mathbf{H}$ NMR (400 MHz, CDCl $\left.{ }_{3}\right) \delta 7.92(\mathrm{dd}, J=3.8,1.2 \mathrm{~Hz}, 1 \mathrm{H}), 7.67(\mathrm{~d}, J=5.0 \mathrm{~Hz}, 1 \mathrm{H}), 7.55-7.52(\mathrm{~m}$, 2H), $7.46-7.45(\mathrm{~m}, 3 \mathrm{H}), 7.17-7.15(\mathrm{~m}, 1 \mathrm{H}) .{ }^{13} \mathbf{C}$ NMR (100 MHz, CDCl $\left.{ }_{3}\right) \delta 182.2,141.5$, $135.2,133.4,131.7,129.8,129.4,128.1,127.0$.

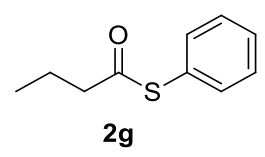

S-Phenyl butanethioate (2g). ${ }^{9}$ Yield 91\% (0.820 g). Colorless oil. ${ }^{1}$ H NMR (400 MHz, CDCl $\left.{ }_{3}\right)$ $\delta 7.41(\mathrm{~s}, 5 \mathrm{H}), 2.65(\mathrm{t}, J=7.3 \mathrm{~Hz}, 2 \mathrm{H}), 1.77(\mathrm{~m}, 2 \mathrm{H}), 1.00(\mathrm{t}, J=7.4 \mathrm{~Hz}, 3 \mathrm{H}) .{ }^{13} \mathbf{C}$ NMR (100 $\left.\underline{\mathbf{M H z}, \mathbf{C D C l}_{3}}\right) \delta 197.6,134.6,129.4,129.3,128.0,45.7,19.3,13.6$.

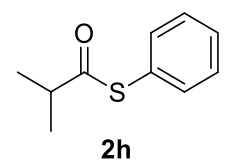

S-Phenyl 2-methylpropanethioate (2h). ${ }^{10}$ Yield 94\% (0.847 g). Light yellow oil. ${ }^{\mathbf{1}}$ H NMR (400 $\underline{\left.\text { MHz, } \mathbf{C D C l}_{\underline{3}}\right)} \delta 7.41(\mathrm{~s}, 5 \mathrm{H}), 2.93-2.82(\mathrm{~m}, 1 \mathrm{H}), 1.28(\mathrm{~d}, J=6.9 \mathrm{~Hz}, 6 \mathrm{H}) .{ }^{{ }^{13} \mathbf{C}} \mathbf{N M R}(\mathbf{1 0 0}$

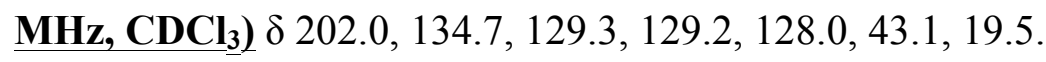




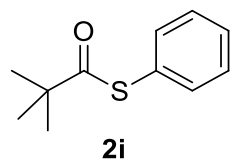

S-Phenyl 2,2-dimethylpropanethioate (2i). ${ }^{11}$ Yield 88\% (0.855 g). Colorless oil. ${ }^{1}$ H NMR (400

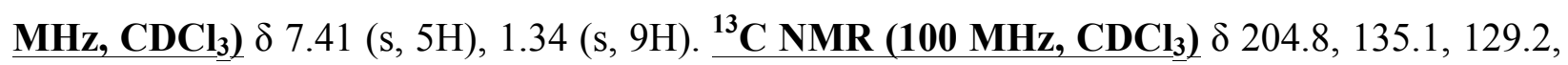
$129.2,128.2,47.0,27.5$.

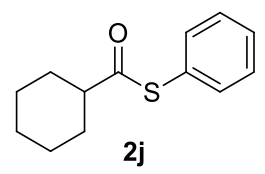

$\boldsymbol{S}$-Phenyl cyclohexanecarbothioate (2j). ${ }^{10}$ Yield 82\% (0.903 g). Colorless oil. ${ }^{\mathbf{1} H}$ NMR (400 $\underline{\left.\mathbf{M H z}, \mathbf{C D C l}_{3}\right)} \delta 7.39(\mathrm{~s}, 5 \mathrm{H}), 2.64-2.57(\mathrm{~m}, 1 \mathrm{H}), 2.02-1.98(\mathrm{~m}, 2 \mathrm{H}), 1.84-1.80(\mathrm{~m}, 2 \mathrm{H})$, $1.69-1.65(\mathrm{~m}, 1 \mathrm{H}), 1.57-1.47(\mathrm{~m}, 2 \mathrm{H}), 1.37-1.20(\mathrm{~m}, 3 \mathrm{H}) .{ }^{13} \mathbf{C} \mathbf{N M R}\left(\mathbf{1 0 0} \mathbf{~ M H z}, \mathbf{C D C l}_{3}\right) \delta$ 200.9, 134.7, 129.3, 129.2, 128.1, 52.6, 29.7, 25.7, 25.6.

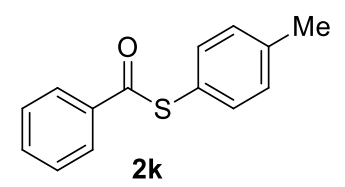

$\boldsymbol{S}$-(p-Tolyl) benzothioate (2k). ${ }^{8}$ Yield 77\% (0.881 g). White solid. ${ }^{1} \mathbf{H}$ NMR (400 MHz, $\left.\mathbf{C D C l}_{3}\right)$ $\delta 8.04(\mathrm{~d}, J=7.0 \mathrm{~Hz}, 2 \mathrm{H}), 7.61(\mathrm{t}, J=7.4 \mathrm{~Hz}, 1 \mathrm{H}), 7.49$ (t, $J=7.7 \mathrm{~Hz}, 2 \mathrm{H}), 7.41(\mathrm{~d}, J=7.9 \mathrm{~Hz}$, 2H), 7.28 (d, $J=7.6 \mathrm{~Hz}, 2 \mathrm{H}), 2.42$ (s, 3H). ${ }^{13} \mathbf{C}$ NMR (100 MHz, $\left.\mathbf{C D C l}_{3}\right) \delta 190.7,139.9,136.8$, $135.2,133.7,130.2,128.8,127.6,123.9,21.5$.

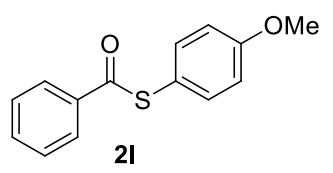

$\boldsymbol{S}$-(4-Methoxyphenyl) benzothioate (2I). ${ }^{8}$ Yield $87 \%$ (1.062 g). White solid. ${ }^{1}$ H NMR (400 MHz, $\left.\mathbf{C D C l}_{3}\right) \delta 8.03(\mathrm{~d}, J=7.3 \mathrm{~Hz}, 2 \mathrm{H}), 7.61(\mathrm{t}, J=7.4 \mathrm{~Hz}, 1 \mathrm{H}), 7.48(\mathrm{t}, J=7.7 \mathrm{~Hz}, 2 \mathrm{H}), 7.44-$ 7.40 (m, 2H), $7.01-6.97$ (m, 2H), 3.85 (s, 3H). $\left.{ }^{13} \mathbf{C ~ N M R ~ ( 1 0 0 ~ M H z , ~ C D C l ~} \underline{3}_{3}\right) \delta 191.2,160.9$, $136.8,133.7,128.9,127.6,118.0,115.1,55.5$. 


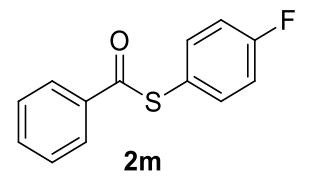

$\boldsymbol{S}$-(4-Fluorophenyl) benzothioate (2m) ${ }^{8}$ Yield $96 \%$ (1.115 g). White solid. ${ }^{1}$ H NMR (400

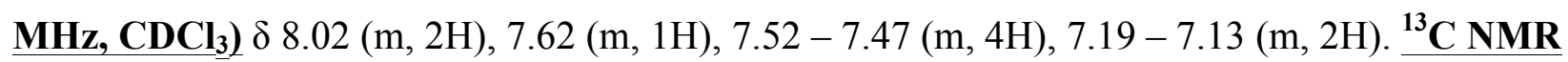
$\left(\mathbf{1 0 0} \mathbf{~ M H z}, \mathbf{C D C l}_{3}\right) \delta 190.3,163.8\left(\mathrm{~d}, J^{l}=250.0 \mathrm{~Hz}\right), 137.3\left(\mathrm{~d}, J^{3}=8.6 \mathrm{~Hz}\right), 136.5,134.0,128.9$, 127.6, $122.7\left(\mathrm{~d}, J^{4}=3.6 \mathrm{~Hz}\right), 116.7\left(\mathrm{~d}, J^{2}=22.3 \mathrm{~Hz}\right) .{ }^{19} \mathbf{F}$ NMR $\left(376 \mathbf{~ M H z}, \mathbf{C D C l}_{3}\right) \delta-111.02$.

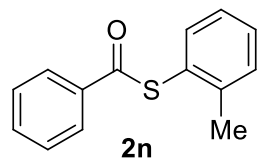

$\boldsymbol{S}$-(o-Tolyl) benzothioate (2n). ${ }^{8}$ Yield 93\% (1.065 g). Colorless oil. ${ }^{1} \mathbf{H}$ NMR (400 MHz, $\left.\mathbf{C D C l}_{3}\right)$ $\delta 8.07(\mathrm{~d}, J=7.5 \mathrm{~Hz}, 2 \mathrm{H}), 7.62(\mathrm{~m}, 1 \mathrm{H}), 7.52-7.48(\mathrm{~m}, 3 \mathrm{H}), 7.41-7.36(\mathrm{~m}, 2 \mathrm{H}), 7.30-7.27$ (m, 1H), 2.41 (s, 3H). ${ }^{13} \mathbf{C}$ NMR (100 MHz, $\left.\mathbf{C D C l}_{3}\right) \delta$ 189.8, 142.8, 136.9, 136.5, 133.7, 131.0, $130.4,128.9,127.7,126.9,126.8,21.0$.

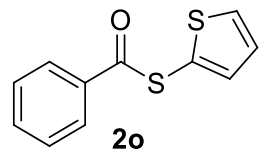

$\boldsymbol{S}$-(Thiophen-2-yl) benzothioate (2o). ${ }^{12}$ Yield 73\% (0.804 g). Brown solid. ${ }^{1}$ H NMR (400 MHz, $\left.\mathbf{C D C l}_{3}\right) \delta 8.02(\mathrm{dd}, J=8.4,1.0 \mathrm{~Hz}, 2 \mathrm{H}), 7.65-7.61(\mathrm{~m}, 2 \mathrm{H}), 7.50(\mathrm{t}, J=7.8 \mathrm{~Hz}, 2 \mathrm{H}), 7.27(\mathrm{dd}, J$ $=3.6,1.2 \mathrm{~Hz}, 1 \mathrm{H}), 7.17(\mathrm{dd}, J=5.3,3.6 \mathrm{~Hz}, 1 \mathrm{H}) .{ }^{13} \mathbf{C} \mathbf{~ N M R}\left(\mathbf{1 0 0} \mathbf{~ M H z}, \mathbf{C D C l}_{3}\right) \delta 190.0,136.5$, $136.1,134.1,132.3,129.0,128.1,127.7,124.3$.

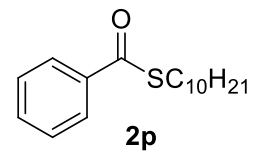

$\boldsymbol{S}$-Decyl benzothioate (2p) ${ }^{8}$ Yield 90\% (1.259 g). Yellow oil. ${ }^{1} \mathbf{H}$ NMR (400 $\left.\mathbf{M H z}, \mathbf{C D C l}_{3}\right) \delta$ $7.97(\mathrm{dd}, J=8.4,1.3 \mathrm{~Hz}, 2 \mathrm{H}), 7.56$ (t, $J=7.4 \mathrm{~Hz}, 1 \mathrm{H}), 7.44$ (t, $J=7.7 \mathrm{~Hz}, 2 \mathrm{H}), 3.07$ (t, $J=8.0$ $\mathrm{Hz}, 2 \mathrm{H}), 1.67$ (p, $J=7.3 \mathrm{~Hz}, 2 \mathrm{H}), 1.42$ (p, $J=6.7 \mathrm{~Hz}, 2 \mathrm{H}), 1.32-1.26$ (m, 12H), 0.88 (t, $J=6.9$ $\mathrm{Hz}, 3 \mathrm{H}) .{ }^{13} \mathbf{C}$ NMR (100 MHz, $\left.\mathbf{C D C l}_{3}\right) \delta 192.3,137.4,133.3,128.7,127.3,32.0,29.7,29.6$, 29.4, 29.3, 29.2, 29.1, 22.8, 14.3. 


\section{Aryl Sulfide Synthesis through Aryl Exchange between Amides and Thioesters}

\section{S-Phenyl benzothioate (2a, Figure 3A)}<smiles>CCC(=O)N(C(C)=O)C(=O)c1ccc(C(F)(F)F)cc1</smiles>

$1 \mathrm{a}$<smiles>O=C(Sc1ccccc1)c1ccccc1</smiles>

2a

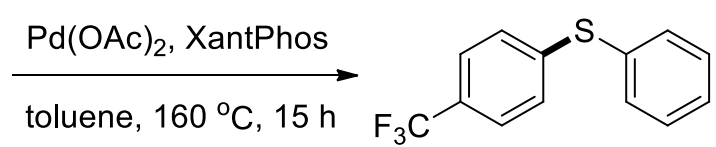

$3 a$

According to the general procedure, the reaction of 1-(4-(trifluoromethyl)benzoyl)piperidine-2,6dione $(0.1 \mathrm{mmol}), S$-phenyl benzothioate $(0.1 \mathrm{mmol}), \mathrm{Pd}(\mathrm{OAc})_{2}(3 \mathrm{~mol} \%)$ and XantPhos $(6$ mol\%) in toluene $(0.20 \mathrm{M})$ for $15 \mathrm{~h}$ at $160{ }^{\circ} \mathrm{C}$, afforded after work-up and chromatography ( silica gel, hexane/DCM = 25/1) the compound (phenyl(4-(trifluoromethyl)phenyl)sulfane (3a) in 92\% yield (23.3 mg). White solid. ${ }^{1} \mathbf{H}$ NMR (400 $\left.\mathbf{~ M H z , ~} \mathbf{C D C l}_{3}\right) \delta 7.49-7.47(\mathrm{~m}, 5 \mathrm{H}), 7.40$ 7.38 (m, 3H), 7.28 (s, 1H). ${ }^{13} \mathbf{C}$ NMR (100 MHz, $\left.\mathbf{C D C l}_{3}\right) \delta 143.0,133.7,132.6,129.8,128.8$, 128.4, $128.2\left(\mathrm{~d}, J^{2}=32.7 \mathrm{~Hz}\right), 126.0\left(\mathrm{q}, J^{3}=3.9 \mathrm{~Hz}\right), 124.2\left(\mathrm{~d}, J^{1}=271.9 \mathrm{~Hz}\right) .{ }^{19} \mathbf{F} \mathbf{N M R}(\mathbf{3 7 6}$ MHz, $\left.\mathbf{C D C l}_{3}\right) \delta-62.47$. The spectral data matched those reported in the literature. ${ }^{8}$

\section{S-Phenyl 4-methoxybenzothioate (2b, Figure 3A)}<smiles>O=C1CCCC(=O)N1C(=O)c1ccc(C(F)(F)F)cc1</smiles>

1a<smiles>COc1ccc(C(=O)Sc2ccccc2)cc1</smiles>

2b

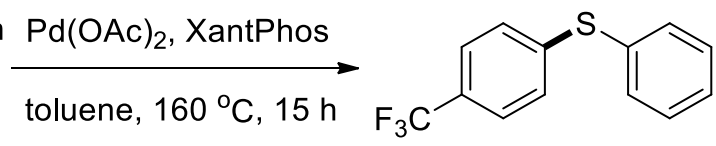

$3 a$

According to the general procedure, the reaction of 1-(4-(trifluoromethyl)benzoyl)piperidine-2,6dione $(0.1 \mathrm{mmol}), S$-phenyl 4-methoxybenzothioate $(0.1 \mathrm{mmol}), \mathrm{Pd}(\mathrm{OAc})_{2}(3 \mathrm{~mol} \%)$ and XantPhos $(6 \mathrm{~mol} \%)$ in toluene $(0.20 \mathrm{M})$ for $15 \mathrm{~h}$ at $160^{\circ} \mathrm{C}$, afforded after work-up and chromatography (silica gel, hexane/DCM = 25/1) the compound (phenyl(4(trifluoromethyl)phenyl)sulfane (3a) in 45\% yield (11.4 mg). White solid. ${ }^{1}$ H NMR (400 MHz, $\left.\left.\underline{\mathbf{C D C l}}_{\mathbf{3}}\right) \delta 7.49-7.47(\mathrm{~m}, 5 \mathrm{H}), 7.40-7.38(\mathrm{~m}, 3 \mathrm{H}), 7.28(\mathrm{~s}, 1 \mathrm{H}) \cdot{ }^{13} \mathbf{C ~ N M R ~ ( 1 0 0 ~ M H z , ~ C D C l} \underline{3}\right) \delta$ 143.0, 133.7, 132.6, 129.8, 128.8, 128.4, 128.2 (d, $\left.J^{2}=32.7 \mathrm{~Hz}\right), 126.0$ (q, $\left.J^{3}=3.9 \mathrm{~Hz}\right), 124.2(\mathrm{~d}$, $\left.J^{l}=271.9 \mathrm{~Hz}\right) .{ }^{19} \mathbf{F}$ NMR (376 $\left.\mathbf{~ M H z}, \mathbf{C D C l}_{3}\right) \delta-62.47$. The spectral data matched those reported in the literature. ${ }^{8}$ 


\section{S-Phenyl 4-fluorobenzothioate (2c, Figure 3A)}<smiles>O=C1CCCC(=O)N1C(=O)c1ccc(C(F)(F)F)cc1</smiles>

1a<smiles>O=C(Sc1ccccc1)c1ccc(F)cc1</smiles>

2c

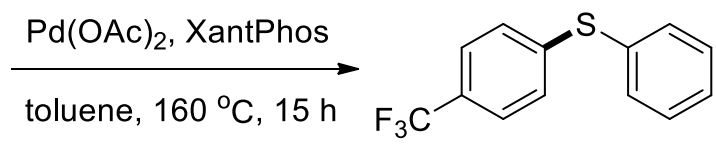

$3 a$

According to the general procedure, the reaction of 1-(4-(trifluoromethyl)benzoyl)piperidine-2,6dione (0.1 mmol), $S$-phenyl 4-fluorobenzothioate (0.1 mmol), $\mathrm{Pd}(\mathrm{OAc})_{2}(3 \mathrm{~mol} \%)$ and XantPhos $(6 \mathrm{~mol} \%)$ in toluene $(0.20 \mathrm{M})$ for $15 \mathrm{~h}$ at $160{ }^{\circ} \mathrm{C}$, afforded after work-up and chromatography (silica gel, hexane/DCM = 25/1) the compound (phenyl(4-(trifluoromethyl)phenyl)sulfane (3a) in 95\% yield (24.1 mg). White solid. ${ }^{1}$ H NMR (400 MHz, CDCl $\left.{ }_{3}\right) \delta 7.49-7.47$ (m, 5H), 7.40 $7.38(\mathrm{~m}, 3 \mathrm{H}), 7.28(\mathrm{~s}, 1 \mathrm{H}) .{ }^{13} \mathbf{C}$ NMR (100 MHz, $\left.\mathbf{C D C l}_{3}\right) \delta 143.0,133.7,132.6,129.8,128.8$, 128.4, $128.2\left(\mathrm{~d}, J^{2}=32.7 \mathrm{~Hz}\right), 126.0\left(\mathrm{q}, J^{3}=3.9 \mathrm{~Hz}\right), 124.2\left(\mathrm{~d}, J^{1}=271.9 \mathrm{~Hz}\right) .{ }^{19} \mathbf{F}$ NMR (376 MHz, $\left.\mathbf{C D C l}_{\underline{3}}\right) \delta-62.47$. The spectral data matched those reported in the literature. ${ }^{8}$

\section{S-Phenyl 4-chlorobenzothioate (2d, Figure 3A)}<smiles>O=C(SPc1ccccc1)c1ccc(Cl)cc1</smiles>

According to the general procedure, the reaction of 1-(4-(trifluoromethyl)benzoyl)piperidine-2,6dione (0.1 mmol), S-phenyl 4-chlorobenzothioate (0.1 mmol), $\mathrm{Pd}(\mathrm{OAc})_{2}(3 \mathrm{~mol} \%)$ and XantPhos $(6 \mathrm{~mol} \%)$ in toluene $(0.20 \mathrm{M})$ for $15 \mathrm{~h}$ at $160{ }^{\circ} \mathrm{C}$, afforded after work-up and chromatography ( ilica gel, hexane/DCM = 25/1) the compound (phenyl(4-(trifluoromethyl)phenyl)sulfane (3a) in 92\% yield (23.3 mg). White solid. ${ }^{1}$ H NMR (400 MHz, $\left.\mathbf{C D C l}_{3}\right) \delta 7.49-7.47$ (m, 5H), 7.40 7.38 (m, 3H), 7.28 (s, 1H). ${ }^{13} \mathbf{C}$ NMR (100 MHz, $\left.\mathbf{C D C l}_{3}\right) \delta 143.0,133.7,132.6,129.8,128.8$, 128.4, $128.2\left(\mathrm{~d}, J^{2}=32.7 \mathrm{~Hz}\right), 126.0\left(\mathrm{q}, J^{3}=3.9 \mathrm{~Hz}\right), 124.2\left(\mathrm{~d}, J^{1}=271.9 \mathrm{~Hz}\right) .{ }^{19} \mathbf{F}$ NMR (376 MHz, $\left.\mathbf{C D C l}_{3}\right) \delta-62.47$. The spectral data matched those reported in the literature. ${ }^{8}$ 


\section{S-Phenyl 2-methylbenzothioate (2e, Figure 3A)}<smiles>O=C1CCCC(=O)N1C(=O)c1ccc(C(F)(F)F)cc1</smiles>

$1 \mathrm{a}$<smiles>Cc1ccccc1C(=O)Sc1ccccc1</smiles>

$2 e$

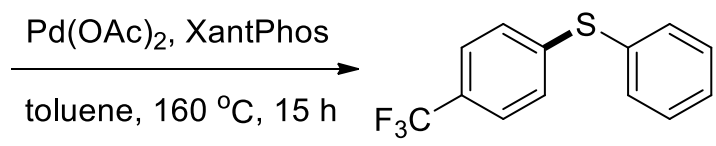

$3 a$

According to the general procedure, the reaction of 1-(4-(trifluoromethyl)benzoyl)piperidine-2,6dione $(0.1 \mathrm{mmol}), S$-phenyl 2-methylbenzothioate $(0.1 \mathrm{mmol}), \mathrm{Pd}(\mathrm{OAc})_{2}(3 \mathrm{~mol} \%)$ and XantPhos $(6 \mathrm{~mol} \%)$ in toluene $(0.20 \mathrm{M})$ for $15 \mathrm{~h}$ at $160{ }^{\circ} \mathrm{C}$, afforded after work-up and chromatography (silica gel, hexane/DCM = 25/1) the compound (phenyl(4(trifluoromethyl)phenyl)sulfane (3a) in 48\% yield (12.2 mg). White solid. ${ }^{1} \mathbf{H}$ NMR (400 MHz, $\left.\underline{\mathbf{C D C l}}_{\mathbf{3}}\right) \delta 7.49-7.47(\mathrm{~m}, 5 \mathrm{H}), 7.40-7.38(\mathrm{~m}, 3 \mathrm{H}), 7.28(\mathrm{~s}, 1 \mathrm{H}) \cdot{ }^{13} \mathbf{C} \mathbf{N M R}(\mathbf{1 0 0} \mathbf{M H z}, \mathbf{C D C l} \underline{3}) \delta$ 143.0, 133.7, 132.6, 129.8, 128.8, 128.4, 128.2 (d, $\left.J^{2}=32.7 \mathrm{~Hz}\right), 126.0$ (q, $\left.J^{3}=3.9 \mathrm{~Hz}\right), 124.2(\mathrm{~d}$, $\left.J^{l}=271.9 \mathrm{~Hz}\right) .{ }^{19} \mathbf{F}$ NMR (376 $\left.\mathbf{~ M H z}, \mathbf{C D C l}_{3}\right) \delta-62.47$. The spectral data matched those reported in the literature. ${ }^{8}$

\section{S-Phenyl thiophene-2-carbothioate (2f, Figure 3A)}<smiles>O=C1CCCC(=O)N1C(=O)c1ccc(C(F)(F)F)cc1</smiles>

$1 \mathrm{a}$<smiles>O=C(SPc1ccccc1)c1cccs1</smiles>

$2 f$

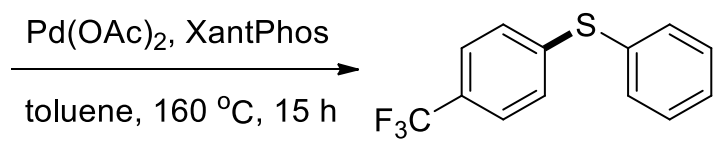

$3 a$

According to the general procedure, the reaction of 1-(4-(trifluoromethyl)benzoyl)piperidine-2,6dione $(0.1 \mathrm{mmol}), S$-phenyl thiophene-2-carbothioate $(0.1 \mathrm{mmol}), \mathrm{Pd}(\mathrm{OAc})_{2}(3 \mathrm{~mol} \%)$ and XantPhos $(6 \mathrm{~mol} \%)$ in toluene $(0.20 \mathrm{M})$ for $15 \mathrm{~h}$ at $160{ }^{\circ} \mathrm{C}$, afforded after work-up and chromatography (silica gel, hexane/DCM = 25/1) the compound (phenyl(4(trifluoromethyl)phenyl)sulfane (3a) in $67 \%$ yield $(17.0 \mathrm{mg})$. White solid. ${ }^{1}$ H NMR (400 MHz, $\left.\underline{\mathbf{C D C l}}_{\mathbf{3}}\right) \delta 7.49-7.47(\mathrm{~m}, 5 \mathrm{H}), 7.40-7.38(\mathrm{~m}, 3 \mathrm{H}), 7.28(\mathrm{~s}, 1 \mathrm{H}) \cdot{ }^{13} \mathbf{C} \mathbf{N M R}(\mathbf{1 0 0 ~ M H z}, \mathbf{C D C l} \underline{3}) \delta$ 143.0, 133.7, 132.6, 129.8, 128.8, 128.4, 128.2 (d, $\left.J^{2}=32.7 \mathrm{~Hz}\right), 126.0$ (q, $\left.J^{3}=3.9 \mathrm{~Hz}\right), 124.2(\mathrm{~d}$, $\left.J^{l}=271.9 \mathrm{~Hz}\right) .{ }^{19} \mathbf{F}$ NMR (376 MHz, $\left.\mathbf{C D C l} 3\right) \delta-62.47$. The spectral data matched those reported in the literature. ${ }^{8}$ 


\section{S-Phenyl butanethioate (2g, Figure 3A)}

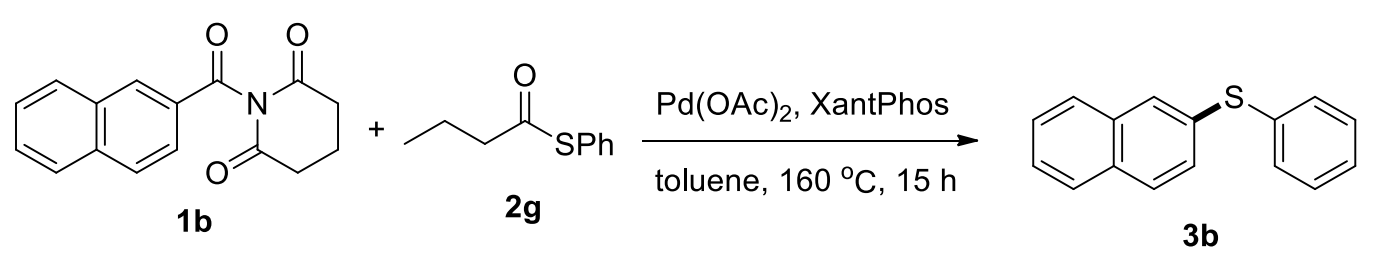

According to the general procedure, the reaction of 1-(2-naphthoyl)piperidine-2,6-dione $(0.1$ mmol), S-phenyl butanethioate $(0.15 \mathrm{mmol}), \mathrm{Pd}(\mathrm{OAc})_{2}(3 \mathrm{~mol} \%)$ and XantPhos $(6 \mathrm{~mol} \%)$ in toluene $(0.20 \mathrm{M})$ for $15 \mathrm{~h}$ at $160{ }^{\circ} \mathrm{C}$, afforded after work-up and chromatography (silica gel, hexane/DCM = 30/1) the compound (naphthalen-2-yl(phenyl)sulfane (3b) in 98\% yield (23.1 mg). White solid. ${ }^{1} \mathbf{H}$ NMR (400 MHz, $\left.\mathbf{C D C l}_{\underline{3}}\right) \delta 7.84$ (s, 1H), 7.81 - 7.72 (m, 3H), 7.49 - 7.44 $(\mathrm{m}, 2 \mathrm{H}), 7.42-7.36(\mathrm{~m}, 3 \mathrm{H}), 7.31(\mathrm{t}, J=7.2 \mathrm{~Hz}, 2 \mathrm{H}), 7.27-7.24(\mathrm{~m}, 1 \mathrm{H}) .{ }^{13} \mathbf{C}$ NMR (100 MHz, $\left.\underline{\mathbf{C D C l}}_{\underline{3}}\right) \delta 136.0,133.9,133.1,132.4,131.1,130.0,129.4,129.0,128.9,127.9,127.6,127.2$, $126.7,126.3$. The spectral data matched those reported in the literature. ${ }^{8}$

\section{$S$-Phenyl 2-methylpropanethioate (2h, Figure 3A)}<smiles>O=C1C[CH+]CCC(=O)N1C(=O)c1ccc2ccccc2c1</smiles>

1b

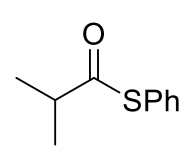

$2 \mathrm{~h}$

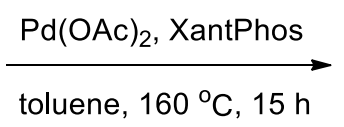

toluene, $160{ }^{\circ} \mathrm{C}, 15 \mathrm{~h}$

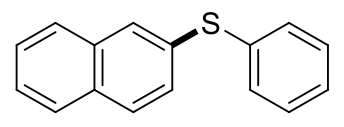

$3 b$

According to the general procedure, the reaction of 1-(2-naphthoyl)piperidine-2,6-dione $(0.1$ mmol), S-phenyl 2-methylpropanethioate $(0.15 \mathrm{mmol}), \mathrm{Pd}(\mathrm{OAc})_{2}(3 \mathrm{~mol} \%)$ and XantPhos (6 $\mathrm{mol} \%$ ) in toluene $(0.20 \mathrm{M})$ for $15 \mathrm{~h}$ at $160{ }^{\circ} \mathrm{C}$, afforded after work-up and chromatography ( silica gel, hexane/DCM = 30/1) the compound (naphthalen-2-yl(phenyl)sulfane (3b) in 90\% yield (21.2 mg). White solid. ${ }^{1}$ H NMR (400 MHz, $\left.\mathbf{C D C l}_{\underline{3}}\right) \delta 7.84$ (s, 1H), $7.81-7.72$ (m, 3H), 7.49 - $7.44(\mathrm{~m}, 2 \mathrm{H}), 7.42$ - $7.36(\mathrm{~m}, 3 \mathrm{H}), 7.31(\mathrm{t}, J=7.2 \mathrm{~Hz}, 2 \mathrm{H}), 7.27$ - $7.24(\mathrm{~m}, 1 \mathrm{H}) .{ }^{13} \mathbf{C ~ N M R}$ $\left(\mathbf{1 0 0 ~ M H z , ~} \mathbf{C D C l}_{3}\right) \delta 136.0,133.9,133.1,132.4,131.1,130.0,129.4,129.0,128.9,127.9,127.6$, $127.2,126.7,126.3$. The spectral data matched those reported in the literature. ${ }^{8}$ 


\section{S-Phenyl 2,2-dimethylpropanethioate (2i, Figure 3A)}<smiles>O=C1C[CH+]CCC(=O)N1C(=O)c1ccc2ccccc2c1</smiles>

$1 b$

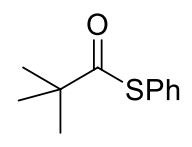

2i

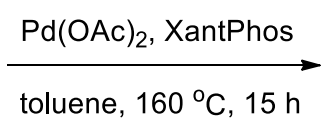

toluene, $160^{\circ} \mathrm{C}, 15 \mathrm{~h}$

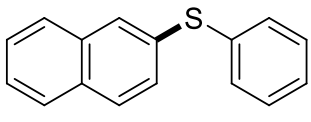

$3 b$

According to the general procedure, the reaction of 1-(2-naphthoyl)piperidine-2,6-dione $(0.1$ mmol), S-phenyl 2,2-dimethylpropanethioate (0.15 mmol), $\mathrm{Pd}(\mathrm{OAc})_{2}(3 \mathrm{~mol} \%)$ and XantPhos (6 $\mathrm{mol} \%$ ) in toluene $(0.20 \mathrm{M})$ for $15 \mathrm{~h}$ at $160{ }^{\circ} \mathrm{C}$, afforded after work-up and chromatography (silica gel, hexane/DCM = 30/1) the compound (naphthalen-2-yl(phenyl)sulfane (3b) in 77\% yield (18.2 mg). White solid. ${ }^{1} \mathbf{H}$ NMR (400 MHz, $\left.\mathbf{C D C l}_{3}\right) \delta 7.84(\mathrm{~s}, 1 \mathrm{H}), 7.81-7.72(\mathrm{~m}, 3 \mathrm{H})$, 7.49 - $7.44(\mathrm{~m}, 2 \mathrm{H}), 7.42-7.36(\mathrm{~m}, 3 \mathrm{H}), 7.31(\mathrm{t}, J=7.2 \mathrm{~Hz}, 2 \mathrm{H}), 7.27$ - $7.24(\mathrm{~m}, 1 \mathrm{H}) .{ }^{13} \mathbf{C ~ N M R}$ $\left(\mathbf{1 0 0} \mathbf{M H z}, \mathbf{C D C l}_{3}\right) \delta 136.0,133.9,133.1,132.4,131.1,130.0,129.4,129.0,128.9,127.9,127.6$, $127.2,126.7,126.3$. The spectral data matched those reported in the literature. ${ }^{8}$

\section{$S$-Phenyl cyclohexanecarbothioate ( $2 \mathrm{j}$, Figure $3 \mathrm{~A})$}<smiles>O=C1C[CH]CCC1=O</smiles>

$1 \mathrm{~b}$<smiles>O=C(c1ccccc1)C1CCCCC1</smiles>

2j

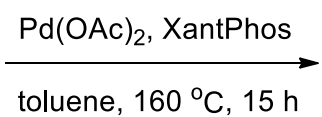

toluene, $160^{\circ} \mathrm{C}, 15 \mathrm{~h}$

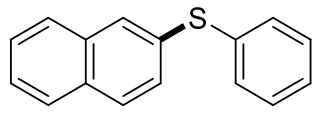

3b

According to the general procedure, the reaction of 1-(2-naphthoyl)piperidine-2,6-dione (0.1 mmol), $S$-phenyl cyclohexanecarbothioate $(0.15 \mathrm{mmol}), \mathrm{Pd}(\mathrm{OAc})_{2}(3 \mathrm{~mol} \%)$ and XantPhos (6 mol\%) in toluene $(0.20 \mathrm{M})$ for $15 \mathrm{~h}$ at $160{ }^{\circ} \mathrm{C}$, afforded after work-up and chromatography ( yield (16.5 mg). White solid. ${ }^{1}$ H NMR (400 MHz, CDCl $\left.{ }_{3}\right) \delta 7.84$ (s, 1H), $7.81-7.72$ (m, 3H), 7.49 - $7.44(\mathrm{~m}, 2 \mathrm{H}), 7.42-7.36(\mathrm{~m}, 3 \mathrm{H}), 7.31(\mathrm{t}, J=7.2 \mathrm{~Hz}, 2 \mathrm{H}), 7.27$ - $7.24(\mathrm{~m}, 1 \mathrm{H}) .{ }^{13} \mathbf{C ~ N M R}$ $\left(\mathbf{1 0 0 ~ M H z , ~} \mathbf{C D C l}_{3}\right) \delta 136.0,133.9,133.1,132.4,131.1,130.0,129.4,129.0,128.9,127.9,127.6$, $127.2,126.7,126.3$. The spectral data matched those reported in the literature. ${ }^{8}$ 


\section{$S$-(p-Tolyl) benzothioate (2k, Figure 3B)}<smiles>O=C1CCCC(=O)N1C(=O)c1ccc(C(F)(F)F)cc1</smiles>

1a<smiles>Cc1ccc(SC(=O)c2ccccc2)cc1</smiles>

2k

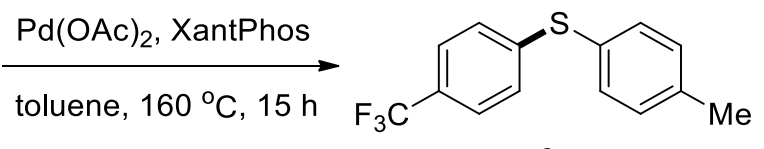

$3 c$

According to the general procedure, the reaction of 1-(4-(trifluoromethyl)benzoyl)piperidine-2,6dione $(0.1 \mathrm{mmol}), S$ - $\left(p\right.$-tolyl) benzothioate $(0.1 \mathrm{mmol}), \mathrm{Pd}(\mathrm{OAc})_{2}(3 \mathrm{~mol} \%)$ and XantPhos (6 $\mathrm{mol} \%)$ in toluene $(0.20 \mathrm{M})$ for $15 \mathrm{~h}$ at $160{ }^{\circ} \mathrm{C}$, afforded after work-up and chromatography ( ilica gel, hexane/DCM = 25/1) the compound ( $p$-tolyl(4-(trifluoromethyl)phenyl)sulfane (3c) in 97\% yield (26.0 mg). White solid. ${ }^{1} \mathbf{H}$ NMR (400 $\left.\mathbf{M H z}^{\mathbf{C}} \mathbf{C D C l}_{3}\right) \delta 7.45$ (d, J = 8.3 Hz, 2H), 7.40 $(\mathrm{d}, \mathrm{J}=8.1 \mathrm{~Hz}, 2 \mathrm{H}), 7.25-7.16(\mathrm{~m}, 4 \mathrm{H}), 2.39$ (s, 3H). ${ }^{13} \mathbf{C}$ NMR (100 MHz, CDCl 3 ) $\delta$ 144.0, 139.4, 134.4, 130.7, 128.4, $127.7\left(\mathrm{~d}, J^{2}=32.6 \mathrm{~Hz}\right), 125.8\left(\mathrm{q}, J^{3}=3.8 \mathrm{~Hz}\right), 124.3\left(\mathrm{~d}, J^{1}=270.1\right.$ $\mathrm{Hz}), 21.4 .{ }^{19} \mathbf{F}$ NMR (376 $\left.\mathbf{M H z}, \mathbf{C D C l}_{3}\right) \delta-62.40$. The spectral data matched those reported in the literature. ${ }^{13}$

\section{S-(4-Methoxyphenyl) benzothioate (2l, Figure 3B)}<smiles>O=C1C[CH+]CCC(=O)N1C(=O)c1ccc(C(F)(F)F)cc1</smiles>

$1 a$

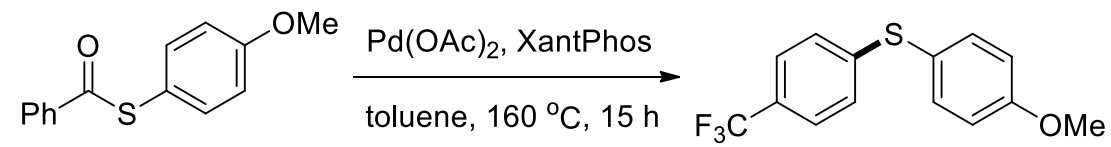

2I 3d

According to the general procedure, the reaction of 1-(4-(trifluoromethyl)benzoyl)piperidine-2,6dione (0.1 mmol), S-(4-methoxyphenyl) benzothioate $(0.1 \mathrm{mmol}), \mathrm{Pd}(\mathrm{OAc})_{2}(3 \mathrm{~mol} \%)$ and XantPhos $(6 \mathrm{~mol} \%)$ in toluene $(0.20 \mathrm{M})$ for $15 \mathrm{~h}$ at $160{ }^{\circ} \mathrm{C}$, afforded after work-up and chromatography (silica gel, hexane/DCM = 25/1) the compound ((4-methoxyphenyl)(4(trifluoromethyl)phenyl)sulfane (3d) in $86 \%$ yield $(24.4 \mathrm{mg})$. White solid. ${ }^{1}$ H NMR (400 MHz, $\left.\underline{\mathbf{C D C l}}_{\mathbf{3}}\right) \delta 7.52-7.38(\mathrm{~m}, 4 \mathrm{H}), 7.14(\mathrm{~d}, J=8.1 \mathrm{~Hz}, 2 \mathrm{H}), 7.00-6.90(\mathrm{~m}, 2 \mathrm{H}), 3.85(\mathrm{~s}, 3 \mathrm{H}) .{ }^{13} \mathbf{C}$ NMR (100 MHz, $\left.\mathbf{C D C l}_{3}\right) \delta 160.8,145.0,136.9,129.8,127.3\left(\mathrm{q}, J^{2}=32.4 \mathrm{~Hz}\right), 126.5,125.8$ (q, $\left.J^{3}=3.9 \mathrm{~Hz}\right), 124.3\left(\mathrm{~d}, J^{l}=270.1 \mathrm{~Hz}\right), 121.7,115.5,55.5 .{ }^{19} \mathbf{F}$ NMR (376 MHz, CDCl 3 ) $\delta-62.34$. The spectral data matched those reported in the literature. ${ }^{14}$ 


\section{$S$-(4-Fluorophenyl) benzothioate (2m, Figure 3B)}<smiles>O=C1CCCC(=O)N1C(=O)c1ccc(C(F)(F)F)cc1</smiles>

1a<smiles>O=C(Sc1ccc(F)cc1)c1ccccc1</smiles>

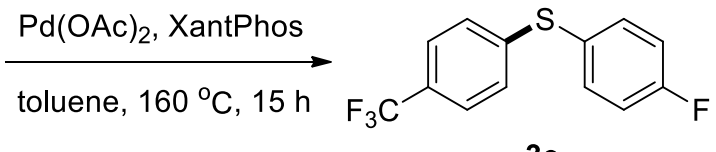

According to the general procedure, the reaction of 1-(4-(trifluoromethyl)benzoyl)piperidine-2,6dione (0.1 mmol), $S$-(4-fluorophenyl) benzothioate $(0.1 \mathrm{mmol}), \mathrm{Pd}(\mathrm{OAc})_{2}(3 \mathrm{~mol} \%)$ and XantPhos $(6 \mathrm{~mol} \%)$ in toluene $(0.20 \mathrm{M})$ for $15 \mathrm{~h}$ at $160{ }^{\circ} \mathrm{C}$, afforded after work-up and chromatography (silica gel, hexane/DCM = 30/1) the compound ((4-fluorophenyl)(4(trifluoromethyl)phenyl)sulfane (3e) in 94\% yield (25.5 mg). White solid. ${ }^{1} \mathbf{H}$ NMR (400 MHz, $\left.\left.\underline{\mathbf{C D C l}}_{\mathbf{3}}\right) \delta 7.52-7.44(\mathrm{~m}, 4 \mathrm{H}), 7.21(\mathrm{~d}, J=7.8 \mathrm{~Hz}, 2 \mathrm{H})\right), 7.15-7.07(\mathrm{~m}, 2 \mathrm{H}) .{ }^{13} \mathbf{C ~ N M R}(\mathbf{1 0 0}$ $\underline{\left.\text { MHz, } \mathbf{C D C l}_{3}\right)} \delta 163.4\left(\mathrm{~d}, J^{2}=248.4 \mathrm{~Hz}\right), 143.4,136.4\left(\mathrm{~d}, J^{5}=8.5 \mathrm{~Hz}\right), 128.1\left(\mathrm{~d}, J^{3}=32.6 \mathrm{~Hz}\right)$, $127.7,137.3\left(\mathrm{~d}, J^{7}=3.4 \mathrm{~Hz}\right), 126.0\left(\mathrm{q}, J^{6}=3.9 \mathrm{~Hz}\right), 124.2\left(\mathrm{~d}, J^{1}=270.1 \mathrm{~Hz}\right), 117.1\left(\mathrm{~d}, J^{4}=21.8\right.$ Hz). ${ }^{19}$ F NMR (376 MHz, $\mathbf{C D C l}_{3}$ ) $\delta-62.47,-111.56$. The spectral data matched those reported in the literature. ${ }^{15}$

\section{$S$-(o-Tolyl) benzothioate (2n, Figure 3B)}<smiles>O=C1C[C+]CCC(=O)N1C(=O)c1ccc(C(F)(F)F)cc1</smiles>

1a<smiles>Cc1ccccc1SC(=O)c1ccccc1</smiles>

$2 \mathrm{n}$

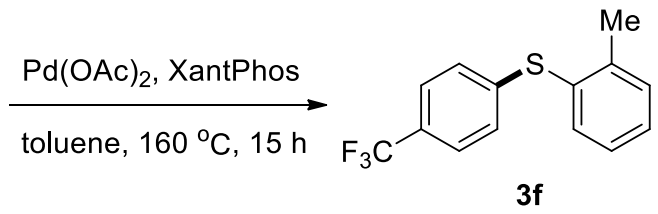

$3 f$

According to the general procedure, the reaction of 1-(4-(trifluoromethyl)benzoyl)piperidine-2,6dione $(0.1 \mathrm{mmol}), S$-(o-tolyl) benzothioate $(0.1 \mathrm{mmol}), \mathrm{Pd}(\mathrm{OAc})_{2}(3 \mathrm{~mol} \%)$ and XantPhos $(6$ $\mathrm{mol} \%)$ in toluene $(0.20 \mathrm{M})$ for $15 \mathrm{~h}$ at $160{ }^{\circ} \mathrm{C}$, afforded after work-up and chromatography ( silica gel, hexane/DCM =20/1) the compound (o-tolyl(4-(trifluoromethyl)phenyl)sulfane (3f) in 98\% yield (26.2 mg). New compound. Colorless oil. ${ }^{1}$ H NMR (400 MHz, CDCl 3 ) $\delta 7.48(\mathrm{~d}, J=$ $7.6 \mathrm{~Hz}, 1 \mathrm{H}), 7.44(\mathrm{~d}, J=8.3 \mathrm{~Hz}, 2 \mathrm{H}), 7.33(\mathrm{~m}, 2 \mathrm{H}), 7.26-7.19(\mathrm{~m}, 1 \mathrm{H}), 7.12(\mathrm{~d}, J=8.2 \mathrm{~Hz}, 2 \mathrm{H})$, 2.37 (s, 3H). ${ }^{13} \mathbf{C}$ NMR (100 MHz, $\left.\mathbf{C D C l}_{3}\right) \delta 143.1,142.1,135.8,131.2,130.7,129.8,127.6(\mathrm{~d}$, $\left.J^{2}=32.4 \mathrm{~Hz}\right), 127.3,127.1,125.9\left(\mathrm{q}, J^{3}=3.7 \mathrm{~Hz}\right), 124.3\left(\mathrm{~d}, J^{l}=270.1 \mathrm{~Hz}\right), 20.8 .{ }^{19} \mathbf{F}$ NMR (376 $\underline{\text { MHz, CDCl}} \underline{3}) \delta-62.35$. HRMS (ESI) $\mathrm{m} / \mathrm{z}$ calcd for $\mathrm{C}_{14} \mathrm{H}_{12} \mathrm{~F}_{3} \mathrm{~S}(\mathrm{M}+\mathrm{H})^{+} 269.0606$, found 269.0610 . 


\section{S-(Thiophen-2-yl) benzothioate (2o, Figure 3B)}<smiles>O=C1C[CH+]CC(=O)N1C(=O)c1ccc(C(F)(F)F)cc1</smiles>

1a<smiles>O=C(Sc1cccs1)c1cccs1</smiles>

20

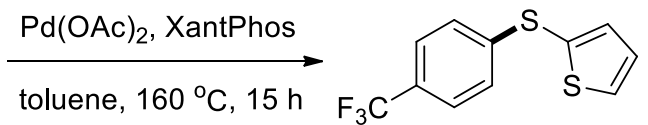

$3 \mathrm{~g}$

According to the general procedure, the reaction of 1-(4-(trifluoromethyl)benzoyl)piperidine-2,6dione (0.1 mmol), $S$-(thiophen-2-yl) benzothioate $(0.1 \mathrm{mmol}), \mathrm{Pd}(\mathrm{OAc})_{2}(3 \mathrm{~mol} \%)$ and XantPhos $(6 \mathrm{~mol} \%)$ in toluene $(0.20 \mathrm{M})$ for $15 \mathrm{~h}$ at $160{ }^{\circ} \mathrm{C}$, afforded after work-up and chromatography ( silica gel, hexane/DCM = 25/1) the compound (2-((4-(trifluoromethyl)phenyl)thio)thiophene (3g) in 80\% yield (20.8 mg). New compound. Light yellow oil. ${ }^{\mathbf{1}}$ H NMR (400 MHz, CDCl 3 ) $\delta$ $7.57(\mathrm{dd}, J=5.4,1.3 \mathrm{~Hz}, 1 \mathrm{H}), 7.47$ (d, $J=8.3 \mathrm{~Hz}, 2 \mathrm{H}), 7.35$ (dd, J = 3.6, $1.3 \mathrm{~Hz}, 1 \mathrm{H}), 7.19$ (d, $J$ $=8.3 \mathrm{~Hz}, 2 \mathrm{H}), 7.14(\mathrm{dd}, J=5.4,3.6 \mathrm{~Hz}, 1 \mathrm{H}) .{ }^{13} \mathbf{C}$ NMR (100 MHz, CDCl$\left.\underline{3}\right) \delta 144.4,137.5$, 132.6, 128.7, 128.5, $127.9\left(\mathrm{~d}, J^{2}=32.5 \mathrm{~Hz}\right), 126.0,125.9\left(\mathrm{q}, J^{3}=3.7 \mathrm{~Hz}\right), 124.2\left(\mathrm{~d}, J^{1}=270.1\right.$ $\mathrm{Hz}) .{ }^{19}$ F NMR (376 MHz, CDCl $\left.\mathbf{l}_{3}\right) \delta-62.40$. HRMS (ESI) $\mathrm{m} / \mathrm{z}$ calcd for $\mathrm{C}_{11} \mathrm{H}_{8} \mathrm{~F}_{3} \mathrm{~S}_{2}(\mathrm{M}+\mathrm{H})^{+}$ 261.0014 , found 261.0012 .

\section{$S$-Decyl benzothioate (2p, Figure 3B)}<smiles>O=C1C[C+]CCC(=O)N1C(=O)c1ccc(C(F)(F)F)cc1</smiles>

1a<smiles>CCCCCCCCCSC(=O)c1ccccc1</smiles>

$2 p$

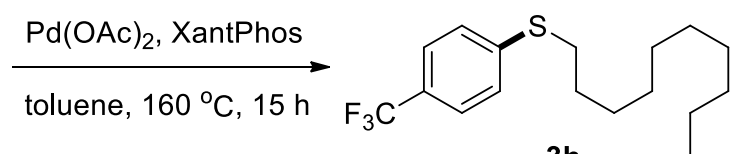

$3 \mathrm{~h}$

According to the general procedure, the reaction of 1-(4-(trifluoromethyl)benzoyl)piperidine-2,6dione (0.1 mmol), $S$-decyl benzothioate $(0.1 \mathrm{mmol}), \mathrm{Pd}(\mathrm{OAc})_{2}(3 \mathrm{~mol} \%)$ and XantPhos $(6 \mathrm{~mol} \%)$ in toluene $(0.20 \mathrm{M})$ for $15 \mathrm{~h}$ at $160{ }^{\circ} \mathrm{C}$, afforded after work-up and chromatography (silica gel, hexane/DCM = 30/1) the (decyl(4-(trifluoromethyl)phenyl)sulfane (3h) compound in 72\% yield $(22.9 \mathrm{mg})$. Colorless oil. ${ }^{1} \mathbf{H}$ NMR (400 MHz, $\left.\mathbf{C D C l}_{3}\right) \delta 7.51(\mathrm{~d}, J=8.2 \mathrm{~Hz}, 2 \mathrm{H}), 7.35(\mathrm{~d}, J=8.3$ $\mathrm{Hz}, 2 \mathrm{H}), 2.97$ (t, $J=7.4 \mathrm{~Hz}, 2 \mathrm{H}), 1.72-1.65$ (m, 2H), $1.48-1.41$ (m, 2H), 1.28 (m, 12H), 0.89 $(\mathrm{t}, J=6.8 \mathrm{~Hz}, 3 \mathrm{H}) .{ }^{13} \mathbf{C} \mathbf{N M R}\left(\mathbf{1 0 0} \mathbf{M H z}, \mathbf{C D C l}_{3}\right) \delta 143.0,127.2(\mathrm{q}, J=32.4 \mathrm{~Hz}), 127.2,127.7$ $\left(\mathrm{q}, J^{3}=3.7 \mathrm{~Hz}\right), 124.4\left(\mathrm{~d}, J^{l}=270.2 \mathrm{~Hz}\right), 32.5,32.0,29.7,29.6,29.5,29.3,29.0,28.9,22.8,14.3$. ${ }^{19}$ F NMR (376 $\left.\mathbf{M H z}, \mathbf{C D C l}_{3}\right) \delta-62.36$. The spectral data matched those reported in the literature. $^{16}$ 


\section{Diphenylsulfane (3i, Figure 4A)}<smiles>O=C1C[CH+]CCC(=O)N1C(=O)c1ccccc1</smiles>

$1 \mathrm{c}$<smiles>O=C(Sc1ccccc1)c1ccc(F)cc1</smiles>

2c

$$
\underset{\text { toluene, } 160^{\circ} \mathrm{C}, 15 \mathrm{~h}}{\stackrel{\mathrm{Pd}(\mathrm{OAc})_{2}, \text { XantPhos }}{\longrightarrow}}
$$<smiles>c1ccc(Sc2ccccc2)cc1</smiles>

$3 \mathbf{i}$

According to the general procedure, the reaction of 1-benzoylpiperidine-2,6-dione (0.1 mmol), $S$ phenyl 4-fluorobenzothioate $(0.1 \mathrm{mmol}), \mathrm{Pd}(\mathrm{OAc})_{2}(3 \mathrm{~mol} \%)$ and XantPhos $(6 \mathrm{~mol} \%)$ in toluene $(0.20 \mathrm{M})$ for $15 \mathrm{~h}$ at $160{ }^{\circ} \mathrm{C}$, afforded after work-up and chromatography (silica gel, hexane) the title compound in 93\% yield (17.3 mg). Yellow solid. ${ }^{1} \mathbf{H}$ NMR (400 MHz, CDCl 2 ) $\delta 7.36-7.33$ (m, 4H), 7.32 - $7.28(\mathrm{~m}, 4 \mathrm{H}), 7.25$ - 7.22 (m, 2H). ${ }^{13} \mathbf{C}$ NMR (100 MHz, CDCl$\left.\underline{3}\right) \delta$ 135.9, 131.2, $129.3,127.2$. The spectral data matched those reported in the literature. ${ }^{8}$

\section{Naphthalen-2-yl(phenyl)sulfane (3b, Figure 4A)}<smiles>O=C1C[CH+]CCC(=O)N1C(=O)c1ccc2ccccc2c1</smiles>

1b<smiles>O=C(Sc1ccccc1)c1ccc(F)cc1</smiles>

2c

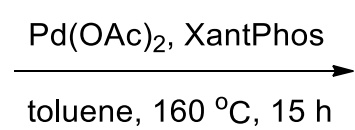
toluene, $160{ }^{\circ} \mathrm{C}, 15 \mathrm{~h}$<smiles>c1ccc(Sc2ccc3ccccc3c2)cc1</smiles>

$3 \mathbf{b}$

According to the general procedure, the reaction of 1-(2-naphthoyl)piperidine-2,6-dione (0.1 mmol), $S$-phenyl 4-fluorobenzothioate $(0.1 \mathrm{mmol}), \mathrm{Pd}(\mathrm{OAc})_{2}(3 \mathrm{~mol} \%)$ and XantPhos (6 mol\%) in toluene $(0.20 \mathrm{M})$ for $15 \mathrm{~h}$ at $160{ }^{\circ} \mathrm{C}$, afforded after work-up and chromatography (silica gel, hexane/DCM $=30 / 1)$ the title compound in $56 \%$ yield $(13.2 \mathrm{mg})$. White solid. ${ }^{1} \mathbf{H}$ NMR (400 $\underline{\left.\text { MHz, } \mathbf{C D C l}_{3}\right)} \delta 7.84(\mathrm{~s}, 1 \mathrm{H}), 7.81-7.72(\mathrm{~m}, 3 \mathrm{H}), 7.49-7.44(\mathrm{~m}, 2 \mathrm{H}), 7.42-7.36(\mathrm{~m}, 3 \mathrm{H}), 7.31$ $(\mathrm{t}, J=7.2 \mathrm{~Hz}, 2 \mathrm{H}), 7.27$ - $7.24(\mathrm{~m}, 1 \mathrm{H}) .{ }^{13} \mathbf{C} \mathbf{N M R}\left(\mathbf{1 0 0} \mathbf{M H z}, \mathbf{C D C l}_{3}\right) \delta 136.0,133.9,133.1$, $132.4,131.1,130.0,129.4,129.0,128.9,127.9,127.6,127.2,126.7,126.3$. The spectral data matched those reported in the literature. ${ }^{8}$ 


\section{Phenyl(p-tolyl)sulfane (3j, Figure 4A)}<smiles>Cc1ccc(C(=O)N2C(=O)C[CH+]CCC2=O)cc1</smiles>

1d<smiles>O=C(Sc1ccccc1)c1ccc(F)cc1</smiles>

2c

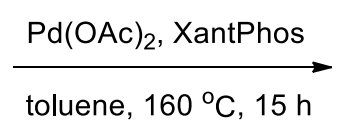

toluene, $160^{\circ} \mathrm{C}, 15 \mathrm{~h}$<smiles>Cc1ccc(Sc2ccccc2)cc1</smiles>

3j

According to the general procedure, the reaction of 1-(4-methylbenzoyl)piperidine-2,6-dione $(0.1$ mmol), $S$-phenyl 4-fluorobenzothioate $(0.1 \mathrm{mmol}), \mathrm{Pd}(\mathrm{OAc})_{2}(3 \mathrm{~mol} \%)$ and XantPhos $(6 \mathrm{~mol} \%)$ in toluene $(0.20 \mathrm{M})$ for $15 \mathrm{~h}$ at $160{ }^{\circ} \mathrm{C}$, afforded after work-up and chromatography (silica gel, hexane) the title compound in $79 \%$ yield $(15.8 \mathrm{mg})$. White solid. ${ }^{1} \mathbf{H}$ NMR (400 MHz, CDCl $\left.\underline{3}\right) \delta$ $7.31(\mathrm{~d}, J=8.1 \mathrm{~Hz}, 2 \mathrm{H}), 7.27(\mathrm{~d}, J=4.4 \mathrm{~Hz}, 4 \mathrm{H}), 7.23-7.18(\mathrm{~m}, 1 \mathrm{H}), 7.15(\mathrm{~d}, J=7.9 \mathrm{~Hz}, 2 \mathrm{H})$,

2.35 (s, 3H). ${ }^{13} \mathbf{C}$ NMR (100 MHz, CDCl 3 ) $\delta 137.7,137.3,132.4,131.4,130.2,129.9,129.2$, $126.5,21.3$. The spectral data matched those reported in the literature. ${ }^{8}$

\section{(4-Methoxyphenyl)(phenyl)sulfane (3k, Figure 4A)}<smiles>COc1ccc(C(=O)N2C(=O)C[CH+]CCC2=O)cc1</smiles>

$1 \mathrm{e}$<smiles>O=C(Sc1ccccc1)c1ccc(F)cc1</smiles>

2c

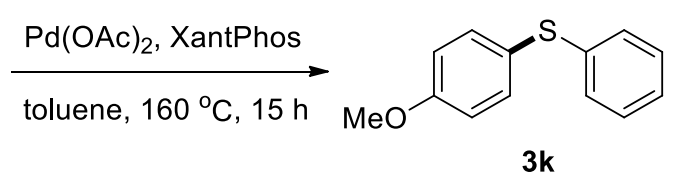

3k

According to the general procedure, the reaction of 1-(4-methoxybenzoyl)piperidine-2,6-dione (0.1 mmol), $S$-phenyl 4-fluorobenzothioate $(0.1 \mathrm{mmol}), \mathrm{Pd}(\mathrm{OAc})_{2}(3 \mathrm{~mol} \%)$ and XantPhos (6 $\mathrm{mol} \%$ ) in toluene $(0.20 \mathrm{M})$ for $15 \mathrm{~h}$ at $160{ }^{\circ} \mathrm{C}$, afforded after work-up and chromatography ( ilica gel, hexane/DCM $=30 / 1)$ the title compound in $98 \%$ yield $\left(21.2 \mathrm{mg}\right.$ ). White solid. ${ }^{1} \mathbf{H}$ NMR (400 MHz, $\left.\mathbf{C D C l}_{3}\right) \delta 7.45-7.41(\mathrm{~m}, 2 \mathrm{H}), 7.27-7.23(\mathrm{~m}, 2 \mathrm{H}), 7.19-7.13(\mathrm{~m}, 3 \mathrm{H}), 6.93$ - 6.89 (m, 2H), 3.83 (s, 3H). ${ }^{13} \mathbf{C}$ NMR (100 MHz, CDCl 3 ) $\delta 160.0,138.7,135.5,129.1,128.3$, $125.9,124.4,115.1,55.5$. The spectral data matched those reported in the literature. ${ }^{8}$ 


\section{Methyl 4-(phenylthio)benzoate (3I, Figure 4A)}<smiles>COC(=O)c1ccc(C(=O)N2C(=O)C[CH+]CC2=O)cc1</smiles><smiles>O=C(Sc1ccccc1)c1ccc(F)cc1</smiles><smiles>COC(=O)c1ccc(Sc2ccccc2)cc1</smiles>

According to the general procedure, the reaction of methyl 4-(2,6-dioxopiperidine-1carbonyl)benzoate (0.1 mmol), $S$-phenyl 4-fluorobenzothioate $(0.1 \mathrm{mmol}), \mathrm{Pd}(\mathrm{OAc})_{2}(3 \mathrm{~mol} \%)$ and XantPhos $(6 \mathrm{~mol} \%)$ in toluene $(0.20 \mathrm{M})$ for $15 \mathrm{~h}$ at $160{ }^{\circ} \mathrm{C}$, afforded after work-up and chromatography (silica gel, hexane/DCM $=20 / 1$ ) the title compound in $73 \%$ yield $(17.8 \mathrm{mg}$ ). White solid. ${ }^{1} \mathbf{H}$ NMR (400 MHz, $\left.\mathbf{C D C l}_{3}\right) \delta 7.89(\mathrm{~d}, J=8.6 \mathrm{~Hz}, 2 \mathrm{H}), 7.50-7.48$ (m, 2H), 7.39 $(\mathrm{dd}, J=5.0,2.0 \mathrm{~Hz}, 3 \mathrm{H}), 7.22-7.19(\mathrm{~m}, 2 \mathrm{H}), 3.89(\mathrm{~s}, 3 \mathrm{H}) .{ }^{13} \mathbf{C}$ NMR (100 MHz, CDCl $\left.{ }_{3}\right) \delta$ $166.8,144.5,133.8,132.5,130.2,129.8,128.8,127.7,127.6,52.2$. The spectral data matched those reported in the literature. ${ }^{8}$

\section{1-(4-(Phenylthio)phenyl)ethan-1-one (3m, Figure 4A)}<smiles>CC(=O)c1ccc(C(=O)N2C(=O)C[CH+]CCC2=O)cc1</smiles>

According to the general procedure, the reaction of methyl 1-(4-acetylbenzoyl)piperidine-2,6dione (0.1 mmol), S-phenyl 4-fluorobenzothioate (0.1 mmol), Pd(OAc) 2 (3 mol\%) and XantPhos $(6 \mathrm{~mol} \%)$ in toluene $(0.20 \mathrm{M})$ for $15 \mathrm{~h}$ at $160{ }^{\circ} \mathrm{C}$, afforded after work-up and chromatography ( ilica gel, hexane/DCM $=25 / 1)$ the title compound in $58 \%$ yield $\left(13.2 \mathrm{mg}\right.$ ). White solid. ${ }^{1} \mathbf{H}$ NMR (400 MHz, CDCl $\left.{ }_{3}\right) \delta 7.82(\mathrm{~d}, J=8.6 \mathrm{~Hz}, 2 \mathrm{H}), 7.51-7.49(\mathrm{~m}, 2 \mathrm{H}), 7.40$ (dd, $J=5.0,1.9$ $\mathrm{Hz}, 3 \mathrm{H}), 7.21(\mathrm{~d}, J=8.6 \mathrm{~Hz}, 2 \mathrm{H}), 2.55$ (s, 3H). ${ }^{13} \mathbf{C}$ NMR (100 MHz, CDCl$\left.{ }_{3}\right) \delta 197.3,145.1$, 134.6, 134.0, 132.2, 129.8, 129.0, 129.0, 127.6, 26.6. The spectral data matched those reported in the literature. ${ }^{8}$ 


\section{(4-Chlorophenyl)(phenyl)sulfane (3n, Figure 4A)}<smiles>O=C1C[CH+]CC(=O)N1C(=O)c1ccc(Cl)cc1</smiles>

$1 \mathrm{~h}$<smiles>O=C(Sc1ccccc1)c1ccc(F)cc1</smiles>

2c

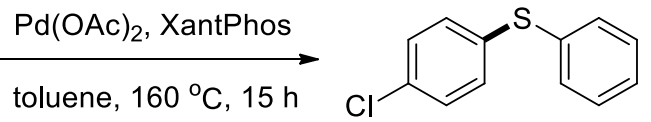

$3 n$

According to the general procedure, the reaction of 1-(4-chlorobenzoyl)piperidine-2,6-dione $(0.1$ mmol), $S$-phenyl 4-fluorobenzothioate $(0.1 \mathrm{mmol}), \mathrm{Pd}(\mathrm{OAc})_{2}(3 \mathrm{~mol} \%)$ and XantPhos $(6 \mathrm{~mol} \%)$ in toluene $(0.20 \mathrm{M})$ for $15 \mathrm{~h}$ at $160{ }^{\circ} \mathrm{C}$, afforded after work-up and chromatography (silica gel, hexane/DCM $=25 / 1)$ the title compound in $77 \%$ yield $(17.0 \mathrm{mg})$. White solid. ${ }^{1} \mathbf{H}$ NMR (400

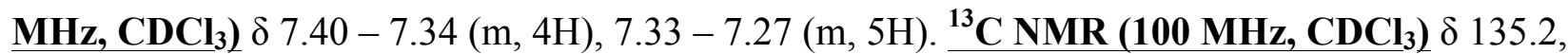
$134.8,133.1,132.2,131.5,129.5,129.4,127.6$. The spectral data matched those reported in the literature. $^{8}$

\section{(3-Chlorophenyl)(phenyl)sulfane (3o, Figure 4A)}<smiles>O=C1C[CH+]CCC(=O)N1C(=O)c1cccc(Cl)c1</smiles>

$1 \mathrm{i}$<smiles>O=C(Sc1ccccc1)c1ccc(F)cc1</smiles>

2c

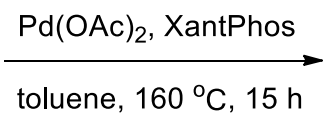

toluene, $160{ }^{\circ} \mathrm{C}, 15 \mathrm{~h}$<smiles>Clc1cccc(Sc2ccccc2)c1</smiles>

30

According to the general procedure, the reaction of 1-(3-chlorobenzoyl)piperidine-2,6-dione $(0.1$ mmol), $S$-phenyl 4-fluorobenzothioate $(0.1 \mathrm{mmol}), \mathrm{Pd}(\mathrm{OAc})_{2}(3 \mathrm{~mol} \%)$ and XantPhos $(6 \mathrm{~mol} \%)$ in toluene $(0.20 \mathrm{M})$ for $15 \mathrm{~h}$ at $160{ }^{\circ} \mathrm{C}$, afforded after work-up and chromatography (silica gel, hexane/DCM $=25 / 1)$ the title compound in 90\% yield $(19.8 \mathrm{mg})$. Colorless oil. ${ }^{1} \mathbf{H}$ NMR (400 $\underline{\left.\mathbf{M H z}, \mathbf{C D C l}_{3}\right)} \delta 7.42-7.39(\mathrm{~m}, 2 \mathrm{H}), 7.38-7.31(\mathrm{~m}, 3 \mathrm{H}), 7.25$ - $7.23(\mathrm{~m}, 1 \mathrm{H}), 7.21-7.14(\mathrm{~m}$, 3H). ${ }^{13} \mathbf{C}$ NMR (100 MHz, CDCl 3 ) $\delta 139.0,135.0,134.0,132.5,130.2,129.6,129.6,128.1$, 128.0, 126.9. The spectral data matched those reported in the literature. ${ }^{17}$ 


\section{Phenyl(o-tolyl)sulfane (3p, Figure 4A)}<smiles>Cc1ccccc1C(=O)N1C(=O)C[CH+]CCC1=O</smiles>

$1 \mathrm{j}$<smiles>O=C(Sc1ccccc1)c1ccc(F)cc1</smiles>

2c

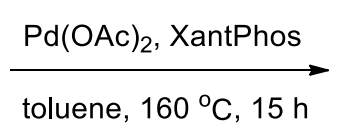

toluene, $160^{\circ} \mathrm{C}, 15 \mathrm{~h}$<smiles>Cc1ccccc1Sc1ccccc1</smiles>

$3 p$

According to the general procedure, the reaction of 1-(2-methylbenzoyl)piperidine-2,6-dione $(0.1$ mmol), $S$-phenyl 4-fluorobenzothioate (0.1 mmol), Pd(OAc) 2 (3 mol\%) and XantPhos (6 mol\%) in toluene $(0.20 \mathrm{M})$ for $15 \mathrm{~h}$ at $160{ }^{\circ} \mathrm{C}$, afforded after work-up and chromatography (silica gel, hexane) the title compound in $87 \%$ yield $(17.4 \mathrm{mg})$. White solid. ${ }^{1} \mathbf{H}$ NMR (400 MHz, CDCl $\left.\underline{\mathbf{3}}\right) \delta$ $7.30-7.14(\mathrm{~m}, 9 \mathrm{H}), 2.38$ (s, 3H). ${ }^{13} \mathbf{C}$ NMR (100 MHz, CDCl 3 ) $\delta 140.1,136.3,133.9,133.1$, $131.2,130.7,129.8,129.3,128.0,126.9,126.5,20.7$. The spectral data matched those reported in the literature. ${ }^{8}$

\section{(2-Fluorophenyl)(phenyl)sulfane (3q, Figure 4A)}<smiles>O=C1C[CH+]CCC(=O)N1C(=O)c1ccccc1F</smiles>

$1 \mathrm{k}$<smiles>O=C(Sc1ccccc1)c1ccc(F)cc1</smiles>

2c

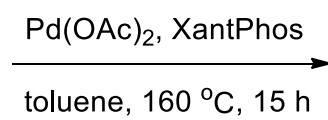

toluene, $160^{\circ} \mathrm{C}, 15 \mathrm{~h}$<smiles>Fc1ccccc1Sc1ccccc1</smiles>

$3 q$

According to the general procedure, the reaction of 1-(2-fluorobenzoyl)piperidine-2,6-dione (0.1 mmol), $S$-phenyl 4-fluorobenzothioate $(0.1 \mathrm{mmol}), \mathrm{Pd}(\mathrm{OAc})_{2}(3 \mathrm{~mol} \%)$ and XantPhos (6 mol\%) in toluene $(0.20 \mathrm{M})$ for $15 \mathrm{~h}$ at $160{ }^{\circ} \mathrm{C}$, afforded after work-up and chromatography (silica gel, hexane/DCM $=30 / 1)$ the title compound in $89 \%$ yield $(18.2 \mathrm{mg})$. White solid. ${ }^{1} \mathbf{H}$ NMR (400 $\underline{\left.\mathbf{M H z}, \mathbf{C D C l}_{3}\right)} \delta 7.36-7.23(\mathrm{~m}, 7 \mathrm{H}), 7.12-7.05(\mathrm{~m}, 2 \mathrm{H}) .{ }^{13} \mathbf{C} \mathbf{N M R}(\mathbf{1 0 0} \mathbf{M H z}, \mathbf{C D C l} \underline{3}) \delta 161.3$ $\left(\mathrm{d}, J^{1}=246.9 \mathrm{~Hz}\right), 134.3,133.6,131.1,129.5\left(\mathrm{~d}, J^{4}=7.9 \mathrm{~Hz}\right), 129.4,127.4,124.9\left(\mathrm{~d}, J^{5}=3.7\right.$ $\mathrm{Hz}), 122.9\left(\mathrm{~d}, J^{3}=17.5 \mathrm{~Hz}\right), 116.1\left(\mathrm{~d}, J^{2}=22.0 \mathrm{~Hz}\right) .{ }^{19} \mathbf{F}$ NMR (376 MHz, CDCl $\left.\underline{3}\right) \delta-108.76$. The spectral data matched those reported in the literature. ${ }^{8}$ 


\section{2-(Phenylthio)furan (3r, Figure 4A)}<smiles>O=C1C[CH+]CCC(=O)N1C(=O)c1ccco1</smiles>

11<smiles>O=C(Sc1ccccc1)c1ccc(F)cc1</smiles>

2c

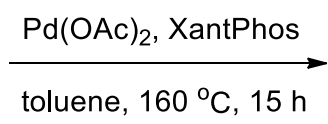

toluene, $160^{\circ} \mathrm{C}, 15 \mathrm{~h}$

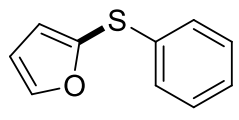

$3 r$

According to the general procedure, the reaction of 1-(furan-2-carbonyl)piperidine-2,6-dione $(0.1$ mmol), $S$-phenyl 4-fluorobenzothioate (0.1 mmol), Pd(OAc) 2 (3 mol\%) and XantPhos (6 mol\%) in toluene $(0.20 \mathrm{M})$ for $15 \mathrm{~h}$ at $160{ }^{\circ} \mathrm{C}$, afforded after work-up and chromatography (silica gel, hexane/DCM $=25 / 1)$ the title compound in $90 \%$ yield $(15.9 \mathrm{mg})$. Colorless oil. ${ }^{1} \mathbf{H}$ NMR (400 $\underline{\left.\mathbf{M H z}, \mathbf{C D C l}_{3}\right)} \delta 7.59(\mathrm{dd}, J=1.9,0.9 \mathrm{~Hz}, 1 \mathrm{H}), 7.36-7.27(\mathrm{~m}, 1 \mathrm{H}), 7.26-7.24(\mathrm{~m}, 1 \mathrm{H}), 7.18-$ $7.15(\mathrm{~m}, 3 \mathrm{H}), 6.75(\mathrm{dd}, J=3.2,0.9 \mathrm{~Hz}, 1 \mathrm{H}), 6.48(\mathrm{dd}, J=3.2,2.0 \mathrm{~Hz}, 1 \mathrm{H}) .{ }^{13} \mathbf{C}$ NMR (100

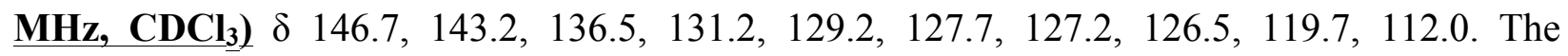
spectral data matched those reported in the literature. ${ }^{18}$

\section{2-(Phenylthio)thiophene (3s, Figure 4A)}<smiles>O=C1C[CH+]CCC(=O)N1C(=O)c1cccs1</smiles>

$1 \mathrm{~m}$<smiles>O=C(Sc1ccccc1)c1ccc(F)cc1</smiles>

2c

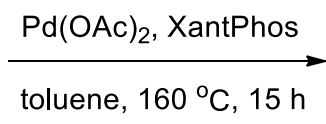

toluene, $160^{\circ} \mathrm{C}, 15 \mathrm{~h}$

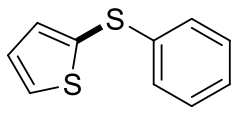

$3 s$

According to the general procedure, the reaction of 1-(thiophene-2-carbonyl)piperidine-2,6-dione (0.1 mmol), $S$-phenyl 4-fluorobenzothioate $(0.1 \mathrm{mmol}), \mathrm{Pd}(\mathrm{OAc})_{2}(3 \mathrm{~mol} \%)$ and XantPhos (6 $\mathrm{mol} \%$ ) in toluene $(0.20 \mathrm{M})$ for $15 \mathrm{~h}$ at $160{ }^{\circ} \mathrm{C}$, afforded after work-up and chromatography ( ilica gel, hexane/DCM $=25 / 1$ ) the title compound in 97\% yield $\left(18.6 \mathrm{mg}\right.$ ). White solid. ${ }^{1} \mathbf{H}$ NMR (400 MHz, $\left.\mathbf{C D C l}_{3}\right) \delta 7.48(\mathrm{dd}, J=5.4,1.2 \mathrm{~Hz}, 1 \mathrm{H}), 7.37-7.28(\mathrm{~m}, 2 \mathrm{H}), 7.24(\mathrm{~s}, 1 \mathrm{H})$, $7.21-7.14(\mathrm{~m}, 3 \mathrm{H}), 7.09(\mathrm{dd}, J=5.4,3.5 \mathrm{~Hz}, 1 \mathrm{H}) .{ }^{13} \mathbf{C}$ NMR (100 MHz, CDCl$\left.{ }_{3}\right) \delta 138.8,136.2$, $131.4,131.2,129.3,129.1,128.1,127.2,126.2$. The spectral data matched those reported in the literature. $^{8}$ 
(E)-Phenyl(styryl)sulfane (3t, Figure 4A)<smiles>O=C(/C=C/c1ccccc1)N1C(=O)C[CH+]CC1=O</smiles>

1n<smiles>O=C(Sc1ccccc1)c1ccc(F)cc1</smiles>

2c

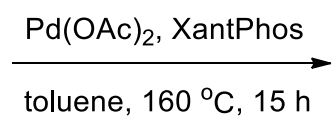

toluene, $160^{\circ} \mathrm{C}, 15 \mathrm{~h}$<smiles>C(=C/c1ccccc1)\Sc1ccccc1</smiles>

3t

According to the general procedure, the reaction of 1-cinnamoylpiperidine-2,6-dione (0.1 mmol), $S$-phenyl 4-fluorobenzothioate $(0.1 \mathrm{mmol}), \mathrm{Pd}(\mathrm{OAc})_{2}(3 \mathrm{~mol} \%)$ and XantPhos $(6 \mathrm{~mol} \%)$ in toluene $(0.20 \mathrm{M})$ for $15 \mathrm{~h}$ at $160{ }^{\circ} \mathrm{C}$, afforded after work-up and chromatography (silica gel, hexane/DCM $=30 / 1)$ the title compound in $88 \%$ yield $(18.7 \mathrm{mg})$. White solid. ${ }^{1} \mathbf{H}$ NMR (400 $\underline{\left.\mathbf{M H z}, \mathbf{C D C l}_{3}\right)} \delta 7.42(\mathrm{~d}, J=7.2 \mathrm{~Hz}, 2 \mathrm{H}), 7.36-7.23(\mathrm{~m}, 8 \mathrm{H}), 6.89(\mathrm{~d}, J=15.5 \mathrm{~Hz}, 1 \mathrm{H}), 6.74(\mathrm{~d}$, $J=15.5 \mathrm{~Hz}, 1 \mathrm{H}) .{ }^{13} \mathbf{C}$ NMR (100 MHz, $\left.\mathbf{C D C l}_{3}\right) \delta 136.7,135.4,132.0,130.0,129.3,128.8$, $127.7,127.1,126.2,123.5$. The spectral data matched those reported in the literature. ${ }^{8}$

\section{1-Benzoylpyrrolidine-2,5-dione (10, Figure 4B)}<smiles>O=C1C[CH+]CC1=O</smiles>

10<smiles>O=C(Sc1ccccc1)c1ccc(F)cc1</smiles>

2c

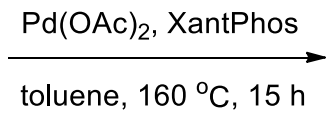

toluene, $160{ }^{\circ} \mathrm{C}, 15 \mathrm{~h}$<smiles>c1ccc(Sc2ccccc2)cc1</smiles>

$3 \mathbf{i}$

According to the general procedure, the reaction of 1-benzoylpyrrolidine-2,5-dione $(0.1 \mathrm{mmol})$, $S$-phenyl 4-fluorobenzothioate $(0.1 \mathrm{mmol}), \mathrm{Pd}(\mathrm{OAc})_{2}(3 \mathrm{~mol} \%)$ and XantPhos $(6 \mathrm{~mol} \%)$ in toluene $(0.20 \mathrm{M})$ for $15 \mathrm{~h}$ at $160{ }^{\circ} \mathrm{C}$, afforded after work-up and chromatography (silica gel, hexane) the compound (diphenylsulfane, 3i) in 60\% yield (11.2 mg). Yellow solid. ${ }^{1} \mathbf{H}$ NMR (400 MHz, CDCl $\left.\underline{3}_{2}\right) \delta 7.36-7.33(\mathrm{~m}, 4 \mathrm{H}), 7.32$ - $7.28(\mathrm{~m}, 4 \mathrm{H}), 7.25$ - $7.22(\mathrm{~m}, 2 \mathrm{H}) .{ }^{13} \mathbf{C}$ NMR $\left(\mathbf{1 0 0 ~ M H z}, \mathbf{C D C l}_{3}\right) \delta 135.9,131.2,129.3,127.2$. The spectral data matched those reported in the literature. $^{8}$ 


\section{Phenyl(1H-pyrrol-1-yl)methanone (1p, Figure 4B)}<smiles>O=C(c1ccccc1)n1cccc1</smiles>

$1 p$<smiles>O=C(Sc1ccccc1)c1ccc(F)cc1</smiles>

2c

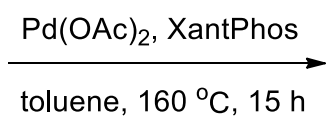

toluene, $160^{\circ} \mathrm{C}, 15 \mathrm{~h}$<smiles>c1ccc(Sc2ccccc2)cc1</smiles>

$3 \mathbf{i}$

According to the general procedure, the reaction of phenyl(1H-pyrrol-1-yl)methanone $(0.1$ mmol), S-phenyl 4-fluorobenzothioate (0.1 mmol), Pd(OAc) 2 (3 mol\%) and XantPhos (6 mol\%) in toluene $(0.20 \mathrm{M})$ for $15 \mathrm{~h}$ at $160{ }^{\circ} \mathrm{C}$, afforded after work-up and chromatography (silica gel, hexane) the compound (diphenylsulfane, $\mathbf{3 i}$ ) in $47 \%$ yield $(8.7 \mathrm{mg})$. Yellow solid. ${ }^{\mathbf{1}} \mathbf{H}$ NMR (400 $\underline{\left.\mathbf{M H z}, \mathbf{C D C l}_{3}\right)} \delta 7.36-7.33(\mathrm{~m}, 4 \mathrm{H}), 7.32-7.28(\mathrm{~m}, 4 \mathrm{H}), 7.25-7.22(\mathrm{~m}, 2 \mathrm{H}) .{ }^{13} \mathbf{C}$ NMR (100 $\underline{\left.\mathbf{M H z}, \mathbf{C D C l}_{3}\right)} \delta 135.9,131.2,129.3,127.2$. The spectral data matched those reported in the literature. $^{8}$

\section{$N$-Phenyl- $N$-tosylbenzamide (1q, Figure 4B)}

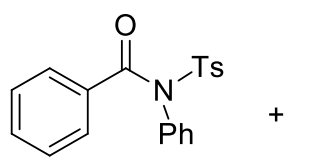

$1 \mathrm{q}$<smiles>O=C(Sc1ccccc1)c1ccc(F)cc1</smiles>

2c

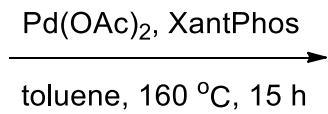

toluene, $160^{\circ} \mathrm{C}, 15 \mathrm{~h}$<smiles>c1ccc(Sc2ccccc2)cc1</smiles>

$3 \mathbf{i}$

According to the general procedure, the reaction of $N$-phenyl- $N$-tosylbenzamide $(0.1 \mathrm{mmol}), S$ phenyl 4-fluorobenzothioate $(0.1 \mathrm{mmol}), \mathrm{Pd}(\mathrm{OAc})_{2}(3 \mathrm{~mol} \%)$ and XantPhos $(6 \mathrm{~mol} \%)$ in toluene $(0.20 \mathrm{M})$ for $15 \mathrm{~h}$ at $160{ }^{\circ} \mathrm{C}$, afforded after work-up and chromatography (silica gel, hexane) the compound (diphenylsulfane, $3 \mathbf{3})$ in $48 \%$ yield $(8.9 \mathrm{mg})$. Yellow solid. ${ }^{1}$ H NMR (400 MHz, $\left.\underline{\mathbf{C D C l}}_{3}\right) \delta 7.36-7.33(\mathrm{~m}, 4 \mathrm{H}), 7.32-7.28(\mathrm{~m}, 4 \mathrm{H}), 7.25-7.22(\mathrm{~m}, 2 \mathrm{H}) .{ }^{13} \mathbf{C}$ NMR (100 MHz, $\left.\mathbf{C D C l}_{3}\right) \delta 135.9,131.2,129.3,127.2$. The spectral data matched those reported in the literature. ${ }^{8}$

\section{$N$-Acetyl- $N$-phenylbenzamide (1r, Figure 4B)}<smiles>O=C(c1ccccc1)N(C(=O)c1ccccc1)c1ccccc1</smiles>

$1 \mathrm{r}$<smiles>O=C(Sc1ccccc1)c1ccc(F)cc1</smiles><smiles>c1ccc(Sc2ccccc2)cc1</smiles>

$3 \mathbf{i}$

According to the general procedure, the reaction of $N$-acetyl- $N$-phenylbenzamide $(0.1 \mathrm{mmol}), S$ phenyl 4-fluorobenzothioate $(0.1 \mathrm{mmol}), \mathrm{Pd}(\mathrm{OAc})_{2}(3 \mathrm{~mol} \%)$ and XantPhos $(6 \mathrm{~mol} \%)$ in toluene $(0.20 \mathrm{M})$ for $15 \mathrm{~h}$ at $160{ }^{\circ} \mathrm{C}$, afforded after work-up and chromatography (silica gel, hexane) the 
compound (diphenylsulfane, $\mathbf{3 i})$ in $50 \%$ yield $(9.3 \mathrm{mg})$. Yellow solid. ${ }^{1} \mathbf{H}$ NMR (400 $\mathbf{~ M H z}$,

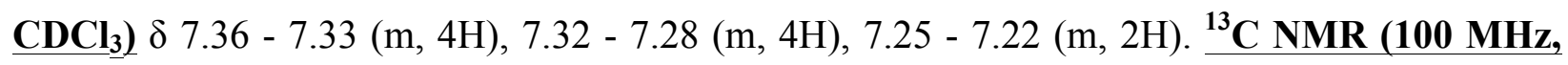
$\left.\mathbf{C D C l}_{3}\right) \delta 135.9,131.2,129.3,127.2$. The spectral data matched those reported in the literature. ${ }^{8}$

\section{$N$-Methyl- $N$-phenylbenzamide (1s, Figure 4B)}

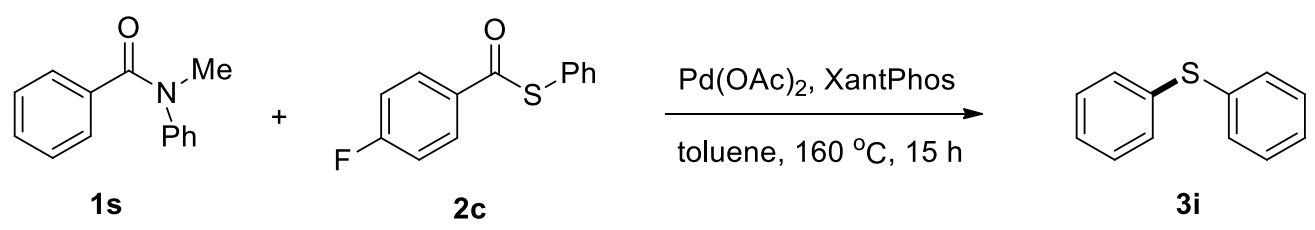

According to the general procedure, the reaction of $N$-methyl- $N$-phenylbenzamide $(0.1 \mathrm{mmol}), S$ phenyl 4-fluorobenzothioate $(0.1 \mathrm{mmol}), \mathrm{Pd}(\mathrm{OAc})_{2}(3 \mathrm{~mol} \%)$ and XantPhos $(6 \mathrm{~mol} \%)$ in toluene $(0.20 \mathrm{M})$ for $15 \mathrm{~h}$ at $160^{\circ} \mathrm{C}$, afforded after work-up and chromatography (silica gel, hexane) the compound (diphenylsulfane, $3 \mathbf{3 i}$ ) in less than $5 \%$ yield.

\section{$N$-Methoxy- $N$-methylbenzamide (1t, Figure 4B)}

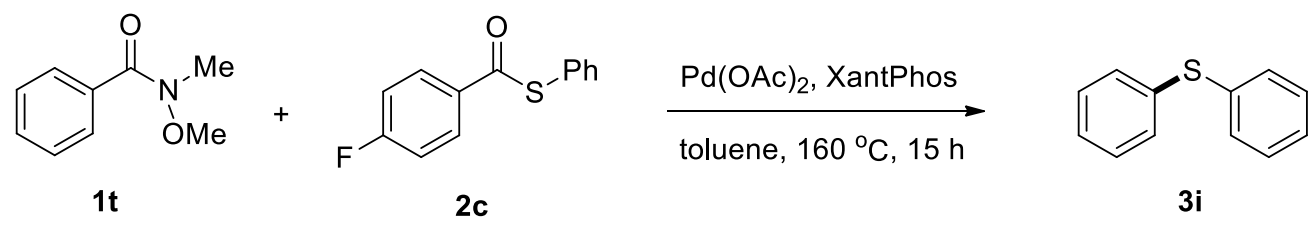

According to the general procedure, the reaction of $N$-methoxy- $N$-methylbenzamide $(0.1 \mathrm{mmol})$, $S$-phenyl 4-fluorobenzothioate $(0.1 \mathrm{mmol}), \mathrm{Pd}(\mathrm{OAc})_{2}(3 \mathrm{~mol} \%)$ and XantPhos $(6 \mathrm{~mol} \%)$ in toluene $(0.20 \mathrm{M})$ for $15 \mathrm{~h}$ at $160{ }^{\circ} \mathrm{C}$, afforded after work-up and chromatography(silica gel, hexane) the compound (diphenylsulfane, $3 \mathbf{3 i}$ ) in less than $5 \%$ yield.

\section{$N, N$-Dimethylbenzamide (1u, Figure 4B).}

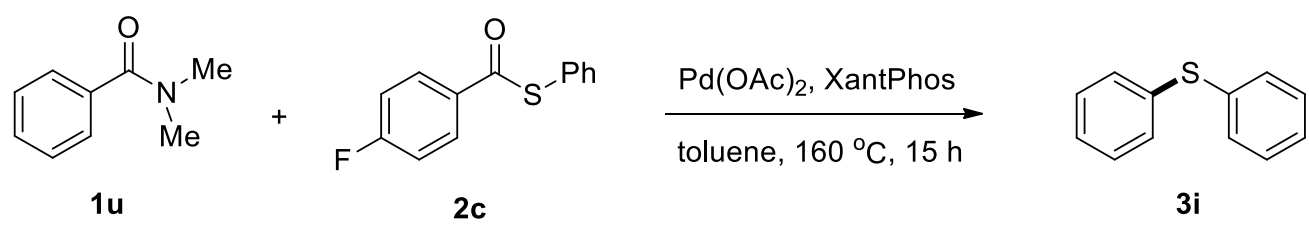

According to the general procedure, the reaction of $N, N$-dimethylbenzamide $(0.1 \mathrm{mmol}), S$ phenyl 4-fluorobenzothioate ( $0.1 \mathrm{mmol}), \mathrm{Pd}(\mathrm{OAc})_{2}(3 \mathrm{~mol} \%)$ and XantPhos (6 mol $\left.\%\right)$ in toluene $(0.20 \mathrm{M})$ for $15 \mathrm{~h}$ at $160{ }^{\circ} \mathrm{C}$, afforded after work-up and chromatography(silica gel, hexane) the compound (diphenylsulfane, $3 \mathbf{3 i}$ ) in less than $5 \%$ yield. 
(6-(3-(Adamantan-1-yl)-4-methoxyphenyl)naphthalen-2-yl)(phenyl)sulfane (3u, Figure 5)

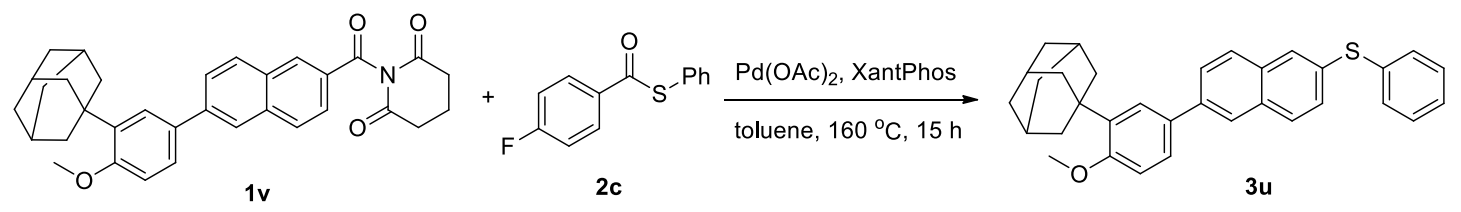

According to the general procedure, the reaction of $1 \mathbf{v}(0.2 \mathrm{mmol}), \mathbf{2 c}(0.2 \mathrm{mmol}), \mathrm{Pd}(\mathrm{OAc})_{2}(3$ mol\%) and XantPhos $(6 \mathrm{~mol} \%)$ in toluene $(0.20 \mathrm{M})$ for $15 \mathrm{~h}$ at $160{ }^{\circ} \mathrm{C}$, afforded after work-up and chromatography ( silica gel, hexane/DCM $=20 / 1)$ the title compound in $72 \%$ yield $(68.6 \mathrm{mg})$. $\left.\underline{\text { New compound. White solid. } \underline{\text { MP }}=141-142{ }^{\circ} \mathrm{C} .{ }^{1} \mathbf{H} \text { NMR (400 MHz, CDCl}} \underline{\mathbf{3}}\right) \delta 7.95(\mathrm{~s}, 1 \mathrm{H})$, $7.86(\mathrm{~s}, 1 \mathrm{H}), 7.84-7.71(\mathrm{~m}, 3 \mathrm{H}), 7.58(\mathrm{~d}, J=2.4 \mathrm{~Hz}, 1 \mathrm{H}), 7.52(\mathrm{dd}, J=8.4,2.4 \mathrm{~Hz}, 1 \mathrm{H}), 7.43$ $(\mathrm{dd}, J=8.6,1.9 \mathrm{~Hz}, 1 \mathrm{H}), 7.40-7.35(\mathrm{~m}, 2 \mathrm{H}), 7.35-7.29(\mathrm{~m}, 2 \mathrm{H}), 7.28-7.25(\mathrm{~m}, 1 \mathrm{H}), 6.99(\mathrm{~d}$, $J=8.4 \mathrm{~Hz}, 1 \mathrm{H}), 3.90(\mathrm{~s}, 3 \mathrm{H}), 2.18(\mathrm{~s}, 6 \mathrm{H}), 2.11(\mathrm{~s}, 3 \mathrm{H}), 1.81(\mathrm{~s}, 6 \mathrm{H}) .{ }^{13} \mathbf{C}$ NMR (100 MHz, $\left.\underline{\mathbf{C D C l}}_{3}\right) \delta 158.8,139.6,139.1,136.3,133.0,132.9,132.7,132.4,130.2,129.4,129.3,129.2$, $127.9,127.1,126.6,126.0,125.7,125.0,112.2,55.3,40.7,37.3,37.3,29.3$. HRMS (ESI) m/z calcd for $\mathrm{C}_{33} \mathrm{H}_{33} \mathrm{OS}[\mathrm{M}+\mathrm{H}]^{+}$477.2247, found 477.2246.

\section{2-i-Butoxy-5-(4-methyl-5-(phenylthio)thiazol-2-yl)benzonitrile (3v, Figure 5)}

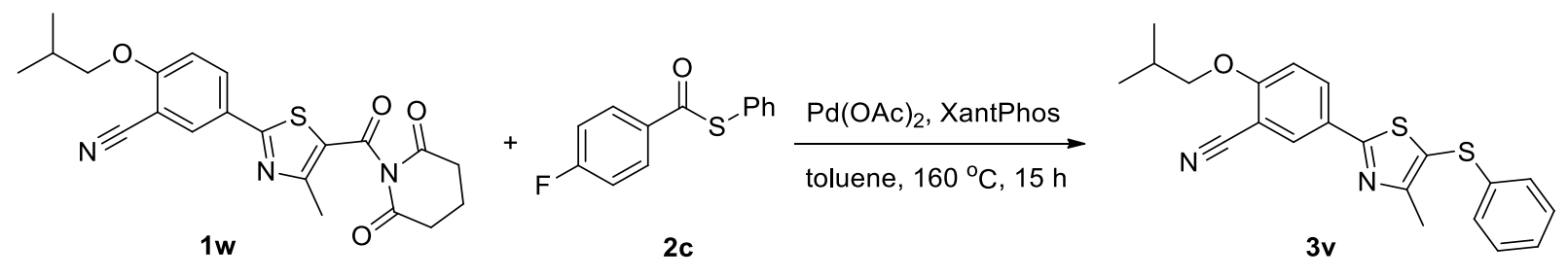

According to the general procedure, the reaction of $\mathbf{1 w}(0.2 \mathrm{mmol}), \mathbf{2 c}(0.2 \mathrm{mmol}), \mathrm{Pd}(\mathrm{OAc})_{2}(3$ $\mathrm{mol} \%$ ) and XantPhos $(6 \mathrm{~mol} \%)$ in toluene $(0.20 \mathrm{M})$ for $15 \mathrm{~h}$ at $160{ }^{\circ} \mathrm{C}$, afforded after work-up and chromatography (silica gel, hexane/DCM $=10 / 1)$ the title compound in $85 \%$ yield $(64.6 \mathrm{mg})$. New compound. Light yellow oil. ${ }^{1} \mathbf{H}$ NMR (400 MHz, $\left.\mathbf{C D C l}_{3}\right) \delta 8.05(\mathrm{~d}, J=2.3 \mathrm{~Hz}, 1 \mathrm{H}), 7.95$ $(\mathrm{dd}, J=8.8,2.3 \mathrm{~Hz}, 1 \mathrm{H}), 7.23-7.18(\mathrm{~m}, 2 \mathrm{H}), 7.14-7.08(\mathrm{~m}, 3 \mathrm{H}), 6.92(\mathrm{~d}, J=8.9 \mathrm{~Hz}, 1 \mathrm{H}), 3.81$ $(\mathrm{d}, J=6.5 \mathrm{~Hz}, 2 \mathrm{H}), 2.45(\mathrm{~s}, 3 \mathrm{H}), 2.13(\mathrm{~m}, 1 \mathrm{H}), 1.02(\mathrm{~d}, J=6.8 \mathrm{~Hz}, 6 \mathrm{H}) .{ }^{13} \mathbf{C}$ NMR (100 MHz, $\left.\underline{\mathbf{C D C l}}_{3}\right) \delta 167.4,162.2,160.1,137.1,132.3,131.8,129.4,127.2,126.6,126.5,120.9,115.7$, 112.7, 103.0, 75.8, 28.3, 19.2, 15.9. HRMS (ESI) $\mathrm{m} / \mathrm{z}$ calcd for $\mathrm{C}_{21} \mathrm{H}_{21} \mathrm{~N}_{2} \mathrm{OS}_{2}[\mathrm{M}+\mathrm{H}]^{+}$ 381.1090 , found 381.1094 . 


\section{2-i-Butoxy-5-(5-((4-methoxyphenyl)thio)-4-methylthiazol-2-yl)benzonitrile (3w, Figure 5)}

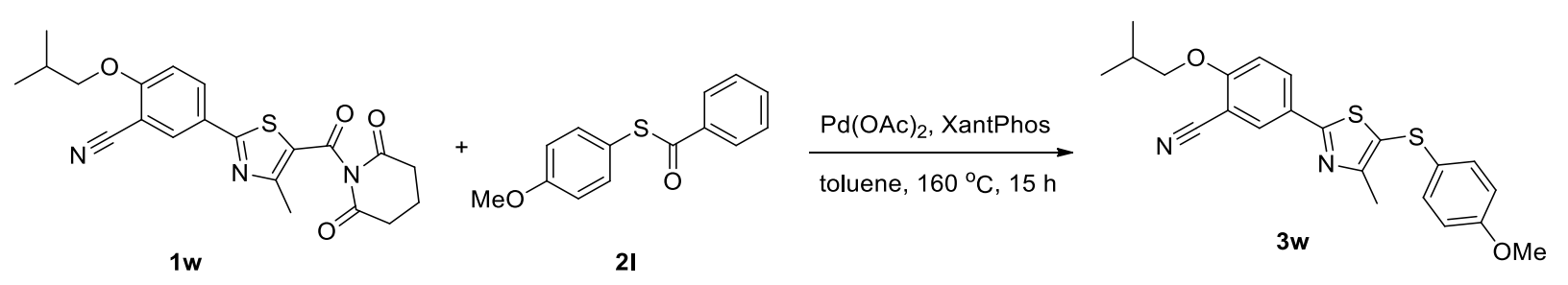

According to the general procedure, the reaction of $\mathbf{1 w}(0.2 \mathrm{mmol}), \mathbf{2 l}(0.2 \mathrm{mmol}), \mathrm{Pd}(\mathrm{OAc})_{2}(3$ $\mathrm{mol} \%$ ) and XantPhos $(6 \mathrm{~mol} \%)$ in toluene $(0.20 \mathrm{M})$ for $15 \mathrm{~h}$ at $160{ }^{\circ} \mathrm{C}$, afforded after work-up and chromatography ( silica gel, hexane/DCM $=10 / 1)$ the title compound in $60 \%$ yield $(49.3 \mathrm{mg})$. $\left.\underline{\text { New compound. Yellow solid. } \underline{\mathbf{M p}}=99-101{ }^{\circ} \mathrm{C} .{ }^{1} \mathbf{H} \text { NMR (400 MHz, CDCl}} \underline{\mathbf{3}}\right) \delta 8.08(\mathrm{~d}, J=2.3$ $\mathrm{Hz}, 1 \mathrm{H}), 7.99$ (dd, $J=8.8,2.3 \mathrm{~Hz}, 1 \mathrm{H}), 7.28-7.25$ (m, 2H), 6.97 (d, $J=8.9 \mathrm{~Hz}, 1 \mathrm{H}), 6.84(\mathrm{~d}, J$ $=8.8 \mathrm{~Hz}, 2 \mathrm{H}), 3.88(\mathrm{~d}, J=6.5 \mathrm{~Hz}, 2 \mathrm{H}), 3.78(\mathrm{~s}, 3 \mathrm{H}), 2.53(\mathrm{~s}, 3 \mathrm{H}), 2.19(\mathrm{~m}, 1 \mathrm{H}), 1.08(\mathrm{~d}, J=6.7$ $\mathrm{Hz}, 6 \mathrm{H}) .{ }^{13} \mathbf{C}$ NMR (100 MHz, $\left.\mathbf{C D C l}_{3}\right) \delta 166.5,162.1,159.3,158.2,132.2,131.7,131.2,127.2$, 126.7, 123.8, 115.7, 115.1, 112.6, 102.9, 75.7, 55.5, 28.3, 19.2, 15.9. HRMS (ESI) $\mathrm{m} / \mathrm{z}$ calcd for $\mathrm{C}_{22} \mathrm{H}_{22} \mathrm{~N}_{2} \mathrm{O}_{2} \mathrm{~S}_{2} \mathrm{Na}[\mathrm{M}+\mathrm{Na}]^{+}$433.1015, found 433.1011.

\section{4-(Phenylthio)- $N, N$-dipropylbenzenesulfonamide (3x, Figure 5)}

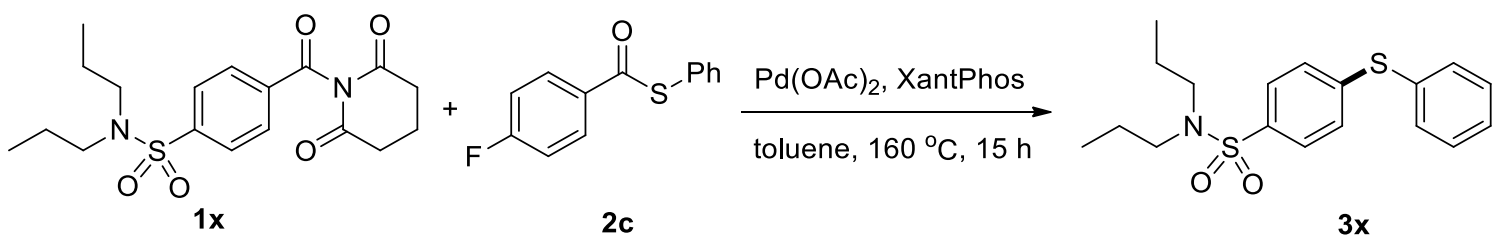

According to the general procedure, the reaction of $1 \mathbf{x}(0.2 \mathrm{mmol}), \mathbf{2 c}(0.2 \mathrm{mmol}), \mathrm{Pd}(\mathrm{OAc})_{2}(3$ $\mathrm{mol} \%$ ) and XantPhos $(6 \mathrm{~mol} \%)$ in toluene $(0.20 \mathrm{M})$ for $15 \mathrm{~h}$ at $160{ }^{\circ} \mathrm{C}$, afforded after work-up and chromatography ( silica gel, hexane/DCM $=20 / 1)$ the title compound in $58 \%$ yield $(40.5 \mathrm{mg})$. White solid. ${ }^{1} \mathbf{H}$ NMR (400 MHz, $\left.\mathbf{C D C l}_{3}\right) \delta 7.66-7.62(\mathrm{~m}, 2 \mathrm{H}), 7.51-7.47(\mathrm{~m}, 2 \mathrm{H}), 7.43-$ $7.39(\mathrm{~m}, 3 \mathrm{H}), 7.23-7.20(\mathrm{~m}, 2 \mathrm{H}), 3.04(\mathrm{t}, J=7.6 \mathrm{~Hz}, 4 \mathrm{H}), 1.57-1.50(\mathrm{~m}, 4 \mathrm{H}), 0.86(\mathrm{t}, J=7.4$ $\mathrm{Hz}, 6 \mathrm{H}) .{ }^{13} \mathbf{C}$ NMR (100 MHz, $\left.\mathbf{C D C l}_{3}\right) \delta 144.2,137.3,134.1,131.8,129.9,129.1,127.8,127.7$, $50.2,22.2,11.3$. The spectral data matched those reported in the literature. ${ }^{19}$ 


\section{3-Methyl-3-((4-(phenylthio)benzyl)oxy)-7,8,9,11,12,13,15,16-octahydro-6H- cyclopenta[a]phenanthren-17(14H)-one (3y, Figure 5)}
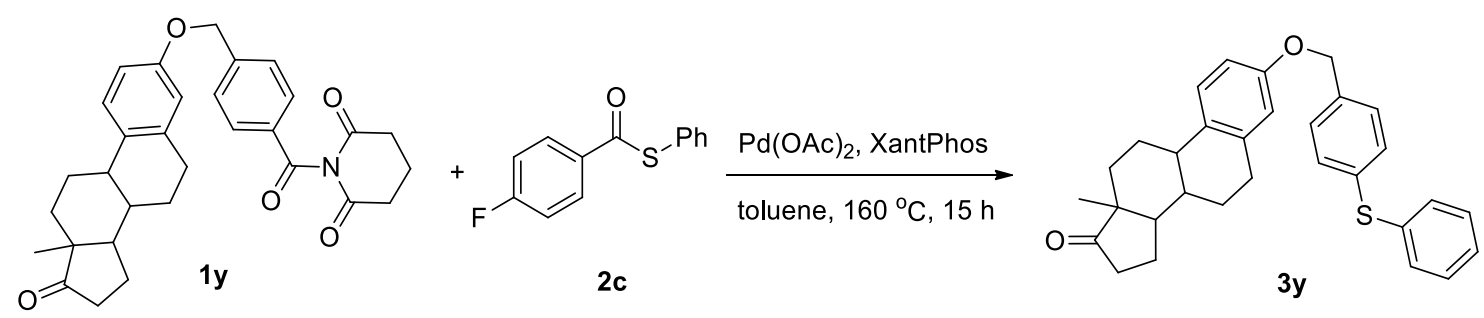

According to the general procedure, the reaction of $1 \mathbf{y}(0.2 \mathrm{mmol}), \mathbf{2 c}(0.2 \mathrm{mmol}), \mathrm{Pd}(\mathrm{OAc})_{2}(3$ $\mathrm{mol} \%$ ) and XantPhos $(6 \mathrm{~mol} \%)$ in toluene $(0.20 \mathrm{M})$ for $15 \mathrm{~h}$ at $160{ }^{\circ} \mathrm{C}$, afforded after work-up and chromatography ( $\left.\underline{\text { New compound. White solid. } \underline{\text { Mp }}=105-107{ }^{\circ} \mathrm{C} .{ }^{1} \mathbf{H} \text { NMR (400 MHz, CDCl}} \underline{\mathbf{3}}\right) \delta 7.35-7.29(\mathrm{~m}$, $8 \mathrm{H}), 7.20(\mathrm{~d}, J=8.5 \mathrm{~Hz}, 1 \mathrm{H}), 7.03(\mathrm{t}, J=8.3 \mathrm{~Hz}, 1 \mathrm{H}), 6.77(\mathrm{~d}, J=8.1 \mathrm{~Hz}, 1 \mathrm{H}), 6.71(\mathrm{~s}, 1 \mathrm{H})$, $5.00(\mathrm{~s}, 2 \mathrm{H}), 2.89(\mathrm{~s}, 2 \mathrm{H}), 2.50\left(\mathrm{dd}, J_{1}=18.6 \mathrm{~Hz}, J_{2}=8.6 \mathrm{~Hz}\right), 2.40-2.38(\mathrm{~m}, 1 \mathrm{H}), 2.25(\mathrm{~s}, 1 \mathrm{H})$, 2.19 - $1.94(\mathrm{~m}, 4 \mathrm{H}), 1.65-1.41(\mathrm{~m}, 6 \mathrm{H}), 0.91$ (s, 3H). ${ }^{13} \mathbf{C}$ NMR (100 MHz, CDCl $\left.\underline{3}\right) \delta 156.8$, 138.0, 136.3, 135.6, 134.4, 132.6, 131.3, 130.1, 129.4, 128.4, 127.3, 126.5, 116.5, 115.0, 112.5, 69.6, 50.5, 48.2, 44.1, 38.5, 36.0, 31.7, 29.8, 26.7, 26.0, 21.7, 14.0. HRMS (ESI) m/z calcd for $\mathrm{C}_{31} \mathrm{H}_{33} \mathrm{O}_{2} \mathrm{~S}[\mathrm{M}+\mathrm{H}]^{+}$469.2196, found 469.2194.

\section{2-i-Butoxy-5-(4-methyl-5-(o-tolylthio)thiazol-2-yl)benzonitrile (3z, Figure 5)}
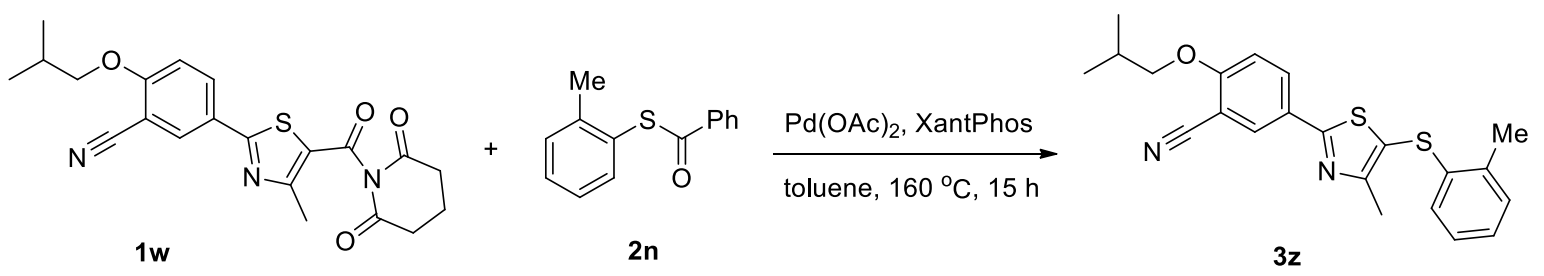

According to the general procedure, the reaction of $\mathbf{1 w}(0.2 \mathrm{mmol}), \mathbf{2 n}(0.2 \mathrm{mmol}), \mathrm{Pd}(\mathrm{OAc})_{2}(3$ mol\%) and XantPhos $(6 \mathrm{~mol} \%)$ in toluene $(0.20 \mathrm{M})$ for $15 \mathrm{~h}$ at $160^{\circ} \mathrm{C}$, afforded after work-up and chromatography (silica gel, hexane/DCM $=10 / 1)$ the title compound in $59 \%$ yield $(46.6 \mathrm{mg})$. New compound. Light yellow oil. ${ }^{1} \mathbf{H}$ NMR (400 MHz, $\left.\mathbf{C D C l}_{3}\right) \delta 8.12(\mathrm{~d}, J=2.3 \mathrm{~Hz}, 1 \mathrm{H}), 8.02$ $(\mathrm{dd}, J=8.9,2.3 \mathrm{~Hz}, 1 \mathrm{H}), 7.21-7.16(\mathrm{~m}, 1 \mathrm{H}), 7.14-7.07(\mathrm{~m}, 2 \mathrm{H}), 6.99(\mathrm{~d}, J=8.9 \mathrm{~Hz}, 1 \mathrm{H}), 6.94$ - $6.90(\mathrm{~m}, 1 \mathrm{H}), 3.89(\mathrm{~d}, J=6.5 \mathrm{~Hz}, 2 \mathrm{H}), 2.50(\mathrm{~s}, 3 \mathrm{H}), 2.44(\mathrm{~s}, 3 \mathrm{H}), 2.20(\mathrm{~m}, 1 \mathrm{H}), 1.09(\mathrm{~d}, J=6.7$ $\mathrm{Hz}, 6 \mathrm{H}) .{ }^{13} \mathbf{C}$ NMR (100 MHz, $\left.\mathbf{C D C l}_{3}\right) \delta 167.3,162.2,160.0,136.1,136.0,132.3,131.8,130.6$, 
126.9, 126.9, 126.7, 126.4, 120.6, 115.7, 112.7, 103.0, 75.7, 28.3, 20.1, 19.2, 15.8. HRMS (ESI) $\mathrm{m} / \mathrm{z}$ calcd for $\mathrm{C}_{22} \mathrm{H}_{23} \mathrm{~N}_{2} \mathrm{OS}_{2}[\mathrm{M}+\mathrm{H}]^{+}$395.1246, found 395.1244.

\section{5-(5-(Decylthio)-4-methylthiazol-2-yl)-2-i-butoxybenzonitrile (3aa, Figure 5)}

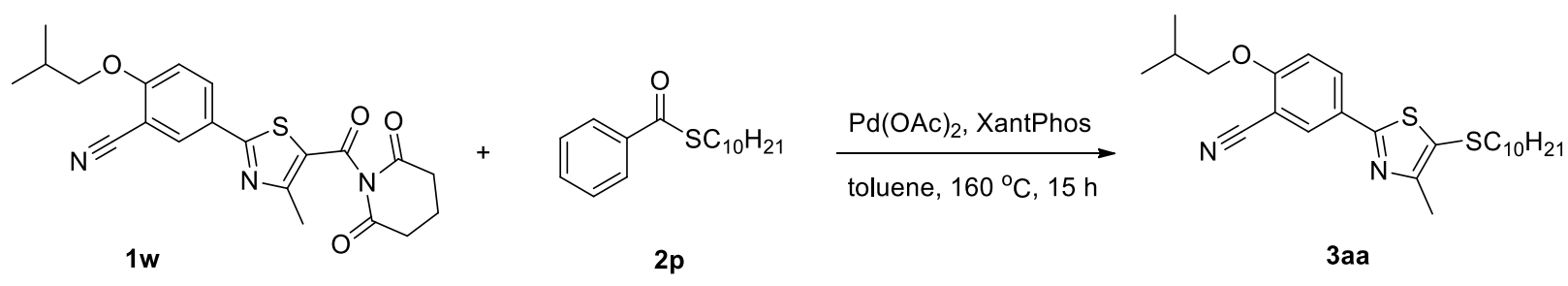

According to the general procedure, the reaction of $\mathbf{1 w}(0.2 \mathrm{mmol}), \mathbf{2} \mathbf{p}(0.2 \mathrm{mmol}), \mathrm{Pd}(\mathrm{OAc})_{2}(3$ mol\%) and XantPhos $(6 \mathrm{~mol} \%)$ in toluene $(0.20 \mathrm{M})$ for $15 \mathrm{~h}$ at $160{ }^{\circ} \mathrm{C}$, afforded after work-up and chromatography (silica gel, hexane/DCM $=10 / 1)$ the title compound in $71 \%$ yield $(63.1 \mathrm{mg}$ ). New compound. Colorless oil. ${ }^{1} \mathbf{H}$ NMR (400 MHz, CDCl $\left.{ }_{3}\right) \delta 8.08(\mathrm{~d}, J=2.3 \mathrm{~Hz}, 1 \mathrm{H}), 8.04-$ 7.99 (m, 1H), 6.98 (d, $J=8.9 \mathrm{~Hz}, 1 \mathrm{H}), 3.88$ (d, $J=6.5 \mathrm{~Hz}, 2 \mathrm{H}), 2.75$ (t, $J=7.3 \mathrm{~Hz}, 2 \mathrm{H}), 2.50$ (s, $3 \mathrm{H}), 2.20(\mathrm{~m}, 1 \mathrm{H}), 1.64-1.56(\mathrm{~m}, 2 \mathrm{H}), 1.39(\mathrm{~m}, 2 \mathrm{H}), 1.29-1.23(\mathrm{~m}, 12 \mathrm{H}), 1.09(\mathrm{~d}, J=6.7 \mathrm{~Hz}$, 6H), $0.87(\mathrm{t}, J=6.8 \mathrm{~Hz}, 3 \mathrm{H}){ }^{13} \mathbf{C}$ NMR (100 MHz, $\left.\mathbf{C D C l}_{3}\right) \delta 165.7,162.0,157.9,132.1,131.7$, 126.9, 124.4, 115.8, 112.6, 102.9, 75.7, 38.7, 32.0, 29.7, 29.6, 29.5, 29.4, 29.3, 28.6, 28.3, 22.8, 19.2, 15.9, 14.3. HRMS (ESI) $\mathrm{m} / \mathrm{z}$ calcd for $\mathrm{C}_{25} \mathrm{H}_{37} \mathrm{~N}_{2} \mathrm{OS}_{2}[\mathrm{M}+\mathrm{H}]^{+} 445.2342$, found 445.2346 . 


\section{Mechanistic Studies (Figure S1)}

Competition Experiments. General Procedure: According to the general procedure, an ovendried vial equipped with a stir bar was charged with two amide or two thioester substrates $(0.10$ $\mathrm{mmol}$, each), $\mathrm{Pd}(\mathrm{OAc})_{2}(0.03$ equiv) and XantPhos ( 0.06 equiv), thioester $(0.05 \mathrm{mmol})$ or amide (0.05 mmol), placed under a positive pressure of argon, and subjected to three evacuation/backfilling cycles under high vacuum. Toluene $(0.20 \mathrm{M})$ was added with vigorous stirring at room temperature, the reaction mixture was placed in a preheated oil bath at $160{ }^{\circ} \mathrm{C}$, and stirred for $15 \mathrm{~h}$ at $160{ }^{\circ} \mathrm{C}$. After the indicated time, the reaction mixture was cooled down to room temperature, diluted with $\mathrm{CH}_{2} \mathrm{Cl}_{2}(10 \mathrm{~mL})$, filtered, and concentrated. A sample was analyzed by ${ }^{1} \mathrm{H}$ NMR $\left(\mathrm{CDCl}_{3}, 400 \mathrm{MHz}\right)$ and/or GC-MS to obtain conversion, selectivity and yield using internal standard and comparison with authentic samples.

Control Experiments. General Procedure: According to the general procedure, an oven-dried vial equipped with a stir bar was charged with a thioester substrate $(0.10 \mathrm{mmol})$, placed under a positive pressure of argon, and subjected to three evacuation/backfilling cycles under high vacuum. Toluene $(0.20 \mathrm{M})$ was added with vigorous stirring at room temperature, the reaction mixture was placed in a preheated oil bath at $160^{\circ} \mathrm{C}$, and stirred for $15 \mathrm{~h}$ at $160^{\circ} \mathrm{C}$. After the indicated time, the reaction mixture was cooled down to room temperature, diluted with $\mathrm{CH}_{2} \mathrm{Cl}_{2}$ $(10 \mathrm{~mL})$, filtered, and concentrated. Alternatively, the same reaction was performed in the presence of $\mathrm{Pd}(\mathrm{OAc})_{2}$ (0.03 equiv) and XantPhos (0.06 equiv). A sample was analyzed by ${ }^{1} \mathrm{H}$ $\mathrm{NMR}\left(\mathrm{CDCl}_{3}, 400 \mathrm{MHz}\right)$ and/or GC-MS to obtain conversion, selectivity and yield using internal standard and comparison with authentic samples.

Overall, the results of competition experiments (Figure S1A-E) indicate that electron-rich amide substrates are inherently more reactive than electron-deficient substrates, while electron-deficient thioesters show higher reactivity than electron-donating substrates. Furthermore, the results of control experiments show that thioesters are recovered unchanged in the absence of catalyst system; however, direct decarbonylation occurs in the absence of amide exchange partner (Figure S1F-G). Further studies on the mechanism, including computational investigations, are underway in our laboratory and will be reported in a due course. 
A. Competition experiment of amides: electron-deficient vs. electron-rich<smiles>O=C1CCCC(=O)N1C(=O)c1ccc(C(F)(F)F)cc1</smiles>

1a, 1.0 equiv<smiles>COc1ccc(C(=O)N2C(=O)CCCC2C)cc1</smiles>

1e, 1.0 equiv

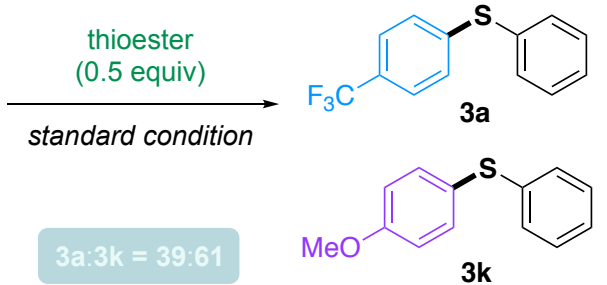

B. Competition experiment of amides: steric hindered vs. regular<smiles>COc1ccccc1C(=O)N1C(=O)CCCC1=O</smiles>

1j, 1.0 equiv<smiles></smiles>

1c, 1.0 equiv

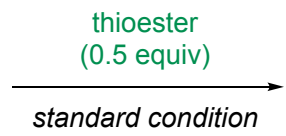

$3 p: 3 i=28: 72$<smiles>Cc1ccccc1Sc1ccccc1</smiles><smiles>c1ccc(Sc2ccccc2)cc1</smiles>

$3 \mathbf{i}$

C. Competition experiment of thioesters: electron-deficient vs. electron-rich<smiles>COc1ccc(SC(=O)c2ccccc2)cc1</smiles>

2q, 1.0 equiv $2 \mathbf{2}, 1.0$ equiv

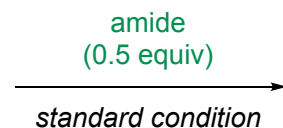

$3 a b: 3 a c=56: 4$<smiles>FC(F)(F)c1ccc(Sc2ccc3ccccc3c2)cc1</smiles><smiles>COc1ccc(Sc2ccc3ccccc3c2)cc1</smiles>

3ac

D. Competition experiment of thioesters: steric hindered vs. regular<smiles>Cc1ccccc1SC(=O)c1ccccc1</smiles><smiles>O=C(Sc1ccccc1)c1ccccc1</smiles>

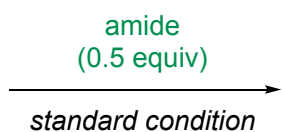<smiles>Cc1ccccc1Sc1ccc2ccccc2c1</smiles>

2n, 1.0 equiv

2a, 1.0 equiv

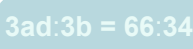

$3 b$

E. Competition experiment of thioesters: aromatic vs. alkyl<smiles>CCCCCCCCSC(=O)c1ccccc1</smiles>

2a, 1.0 equiv $2 \mathbf{p}, 1.0$ equiv

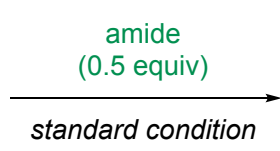

$3 b: 3 a e=97: 3$<smiles>c1ccc(Sc2ccc3ccccc3c2)cc1</smiles><smiles>CCCCCCCCCCSc1ccc2ccccc2c1</smiles>

3ae

Figure S1. Selectivity and Control Experiments in Pd-Catalyzed Aryl Exchange between Amides and Thioesters. 
F. Intramolecular decarbonylation of thioester without catalyst

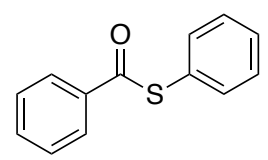

2a toluene, $160^{\circ} \mathrm{C}, 15 \mathrm{~h}$

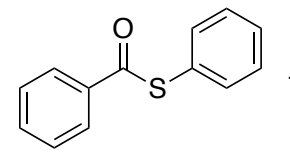

2a: $99 \%$ recovered

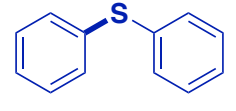

3i: $<1 \%$ yield

G. Palladium-catalyzed intramolecular decarbonylation of thioester<smiles>O=C(Sc1ccccc1)c1ccccc1</smiles>

2a

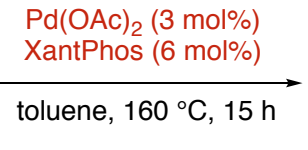

toluene, $160^{\circ} \mathrm{C}, 15 \mathrm{~h}$

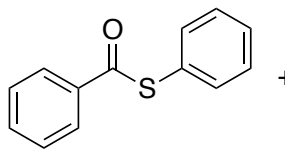

2a: $<1 \%$ yield

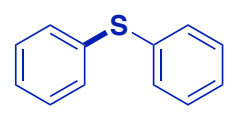

3i: $99 \%$ yield

Figure S1 cont. Selectivity and Control Experiments in Pd-Catalyzed Aryl Exchange between Amides and Thioesters. 


\section{References}

[1] Liu, C.; Szostak, M. Angew. Chem. Int. Ed., 2017, 56, 12718.

[2] Meng, G.; Szostak, M. Org. Lett., 2015, 17, 4364.

[3] Meng, G.; Szostak, M. Org. Lett., 2016, 18, 796.

[4] Idris, M.; Lee, S. Org. Lett., 2020, 22, 9190.

[5] Meng, G.; Szostak, R.; Szostak, M. Org. Lett., 2017, 19, 3596.

[6] Liu, C.; Achtenhagen, M.; Szostak, M. Org. Lett., 2016, 18, 2375.

[7] Gao, P., Szostak, M. Org. Lett., 2020, 22, 6010.

[8] Liu, C.; Szostak, M. Chem. Commun., 2018, 54, 2130.

[9] Kwon, Y.; La, M. T.; Kim, H. New J. Chem., 2018, 42, 10833.

[10] Oost, R.; Misale, A.; Maulide, N. Angew. Chem. Int. Ed., 2016, 55, 4587.

[11] Azeredo, J. B.; Godoi, M.; Schwab, R. S.; Botteselle, G. V.; Braga, A. L. Eur. J. Org. Chem., 2013, 5188.

[12] Skácel, J.; Dračínský, M.; Janeba, Z. J. Org. Chem., 2020, 85, 788.

[13] Liu, D.; Ma, H.; Fang, P.; Mei, T. Angew. Chem. Int. Ed., 2019, 58, 5033.

[14] Escobar, R. A.; Johannes, J. W. Chem. Eur. J., 2020, 26, 5168.

[15] Dayal, S. K.; Taft, R. W. J. Am. Chem. Soc., 1973, 95, 17, 5595.

[16] Timpa, S. D.; Pell, C. J.; Ozerov, O. V. J. Am. Chem. Soc., 2014, 136, 14772.

[17] Xu, H.; Zhao, Y.; Feng, T.; Feng, Y. J. Org. Chem., 2012, 77, 2878.

[18] Zhu, F.; Rodriguez, J.; O’Neill, S.; Walczak, M. A. ACS Cent. Sci., 2018, 4, 1652.

[19] Ichiishi, N.; Malapit, C. A.; Woźniak, Ł.; Sanford, M. S. Org. Lett., 2018, 20, 44. 


\section{${ }^{1} \mathrm{H},{ }^{13} \mathrm{C}$ and ${ }^{19} \mathrm{~F}$ NMR Spectra of amides}
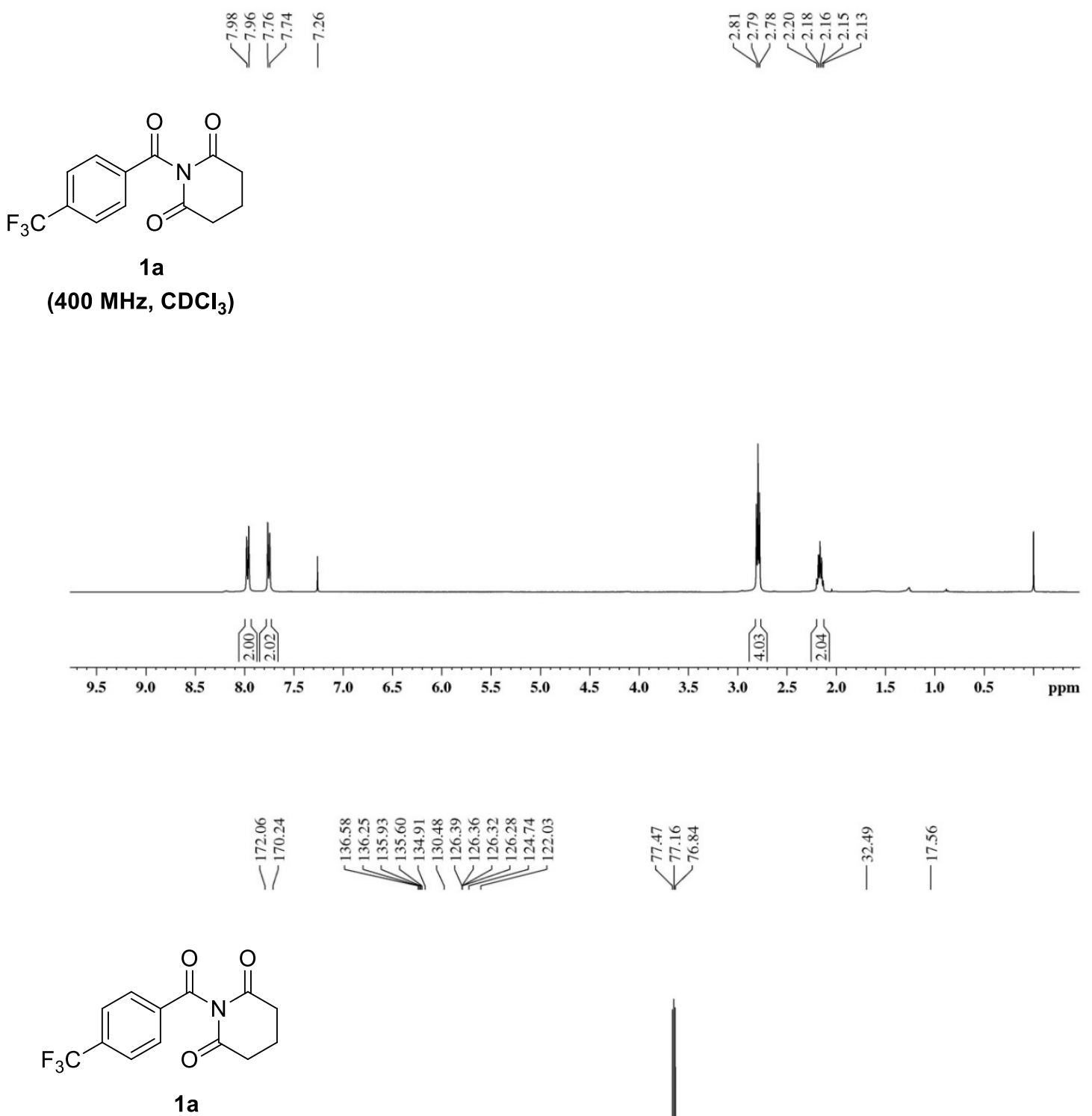

$\left(100 \mathrm{MHz}, \mathrm{CDCl}_{3}\right)$

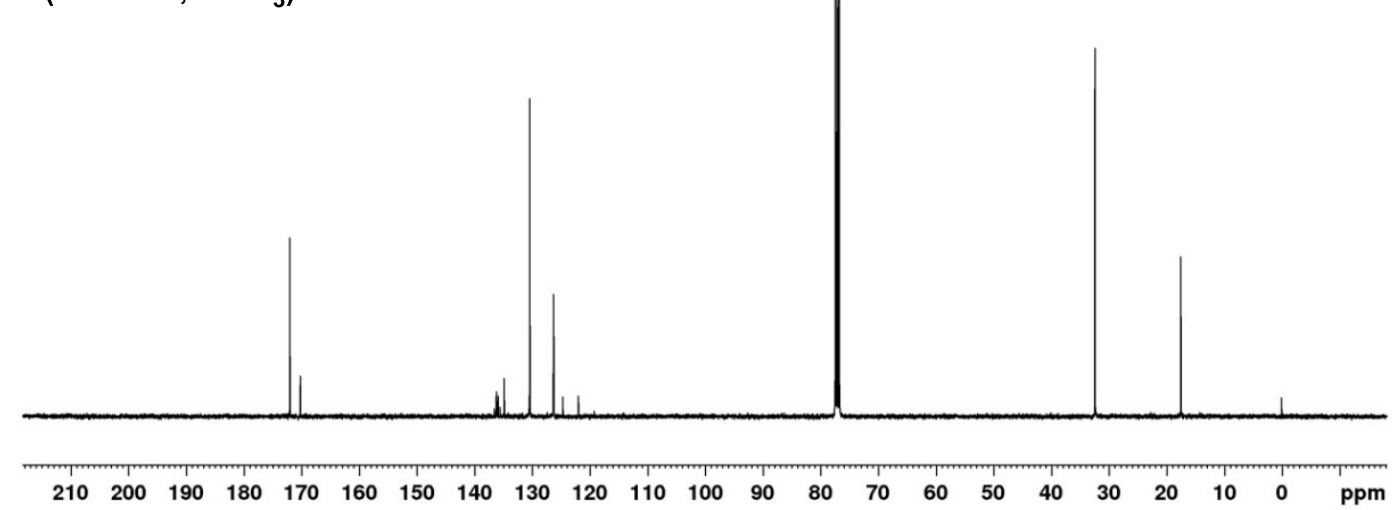




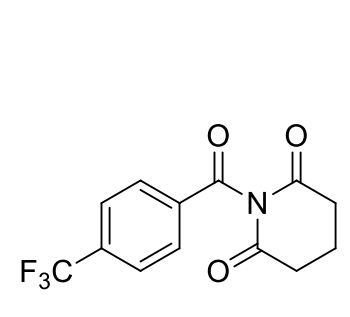

1a

(376 $\mathrm{MHz}, \mathrm{CDCl}_{3}$ )

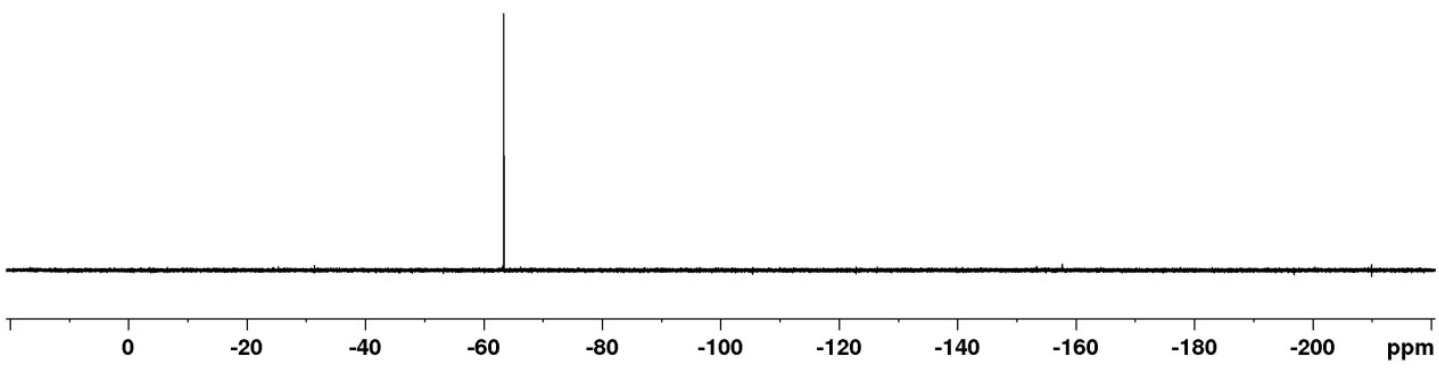



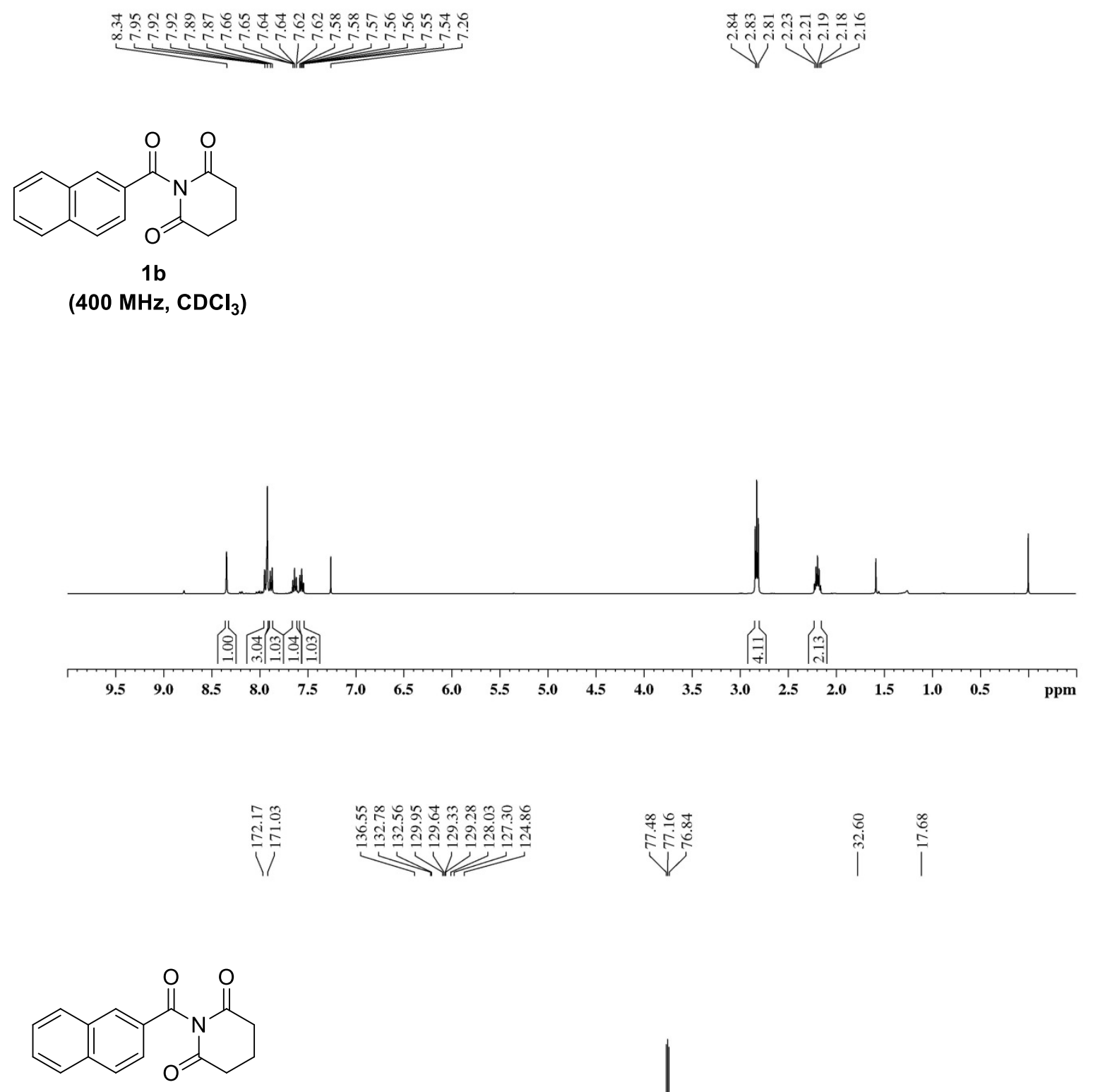

$1 \mathrm{~b}$

$\left(100 \mathrm{MHz}, \mathrm{CDCl}_{3}\right)$

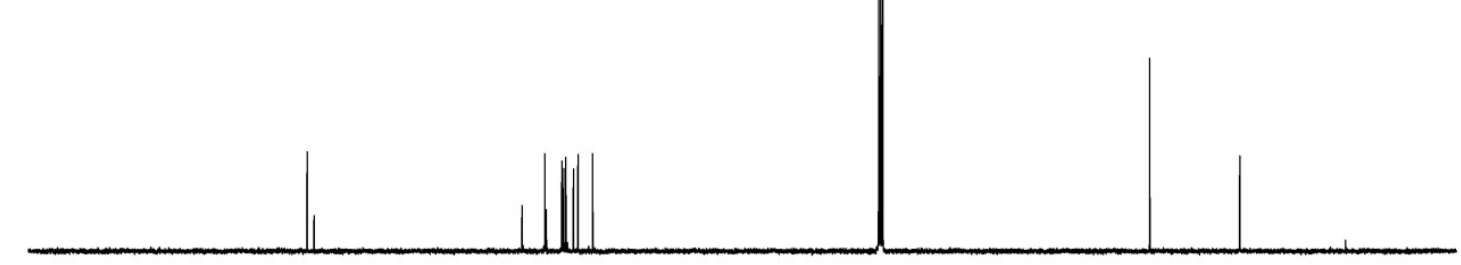

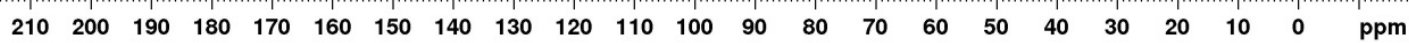



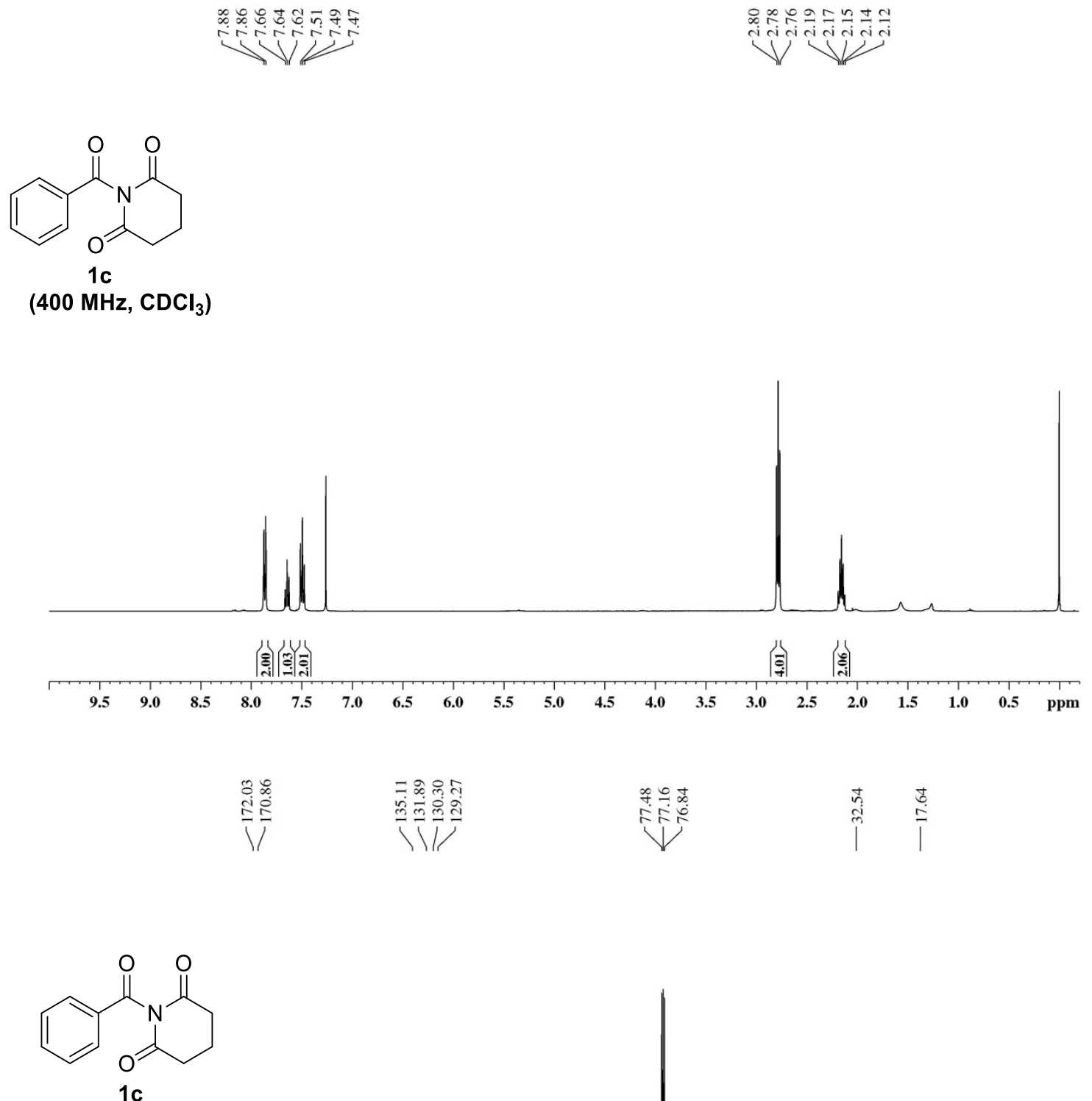

$\left(100 \mathrm{MHz}, \mathrm{CDCl}_{3}\right)$

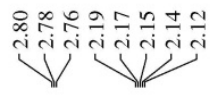

$\left(100 \mathrm{MHz}_{2} \mathrm{CDCl}_{3}\right)$

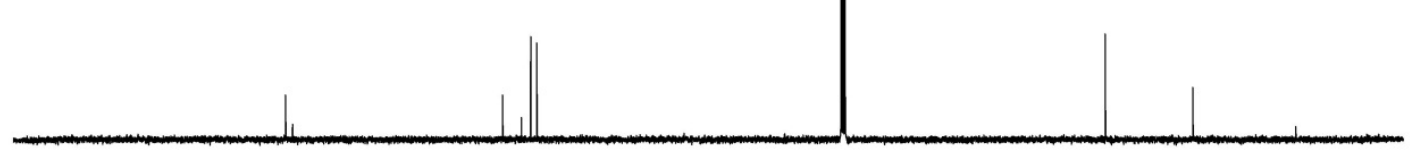

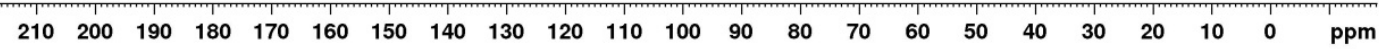




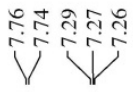

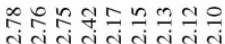

पV<smiles>Cc1ccc(C(=O)N2C(=O)CCCC2=O)cc1</smiles>

1d

(400 $\mathrm{MHz}, \mathrm{CDCl}_{3}$ )

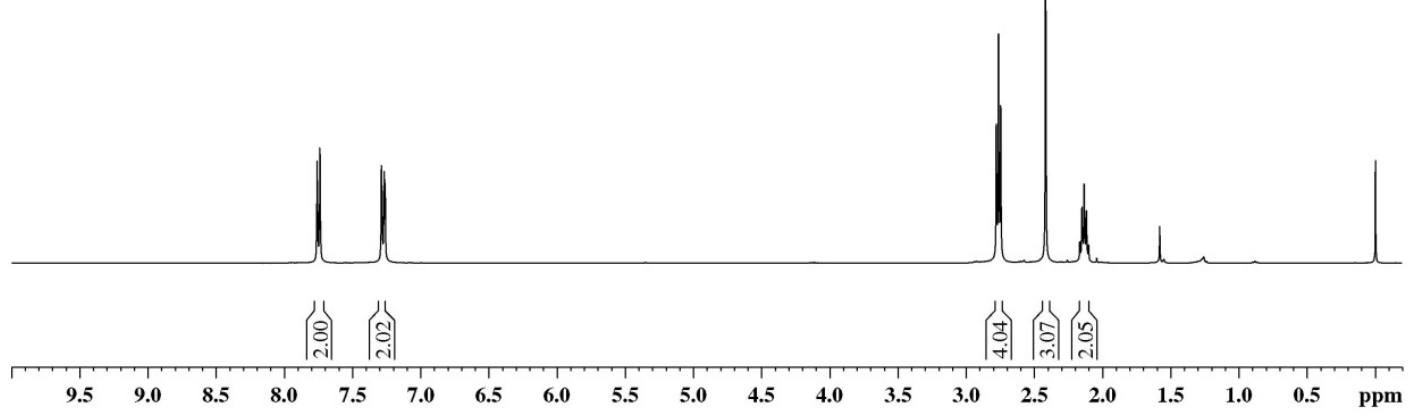

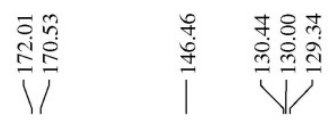
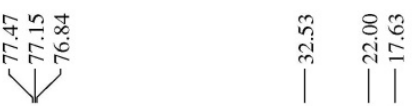<smiles>Cc1ccc(C(=O)N2C(=O)CCCC2=O)cc1</smiles>

1d

$\left(100 \mathrm{MHz} \mathrm{CDCl}_{3}\right)$

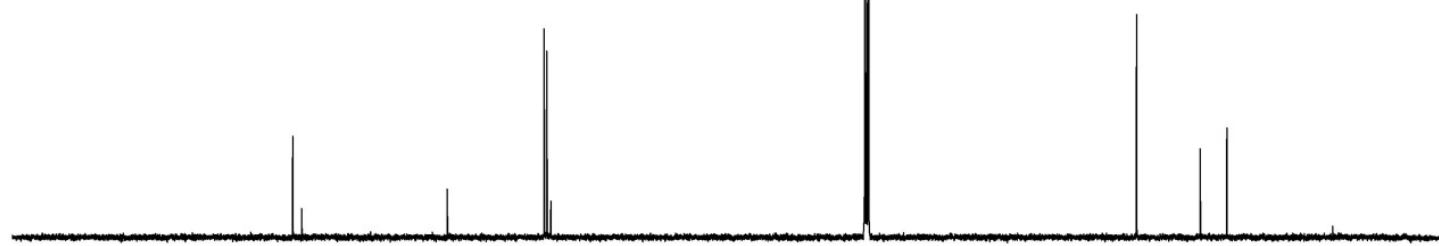

$\begin{array}{llllllllllllllllllllllll}210 & 200 & 190 & 180 & 170 & 160 & 150 & 140 & 130 & 120 & 110 & 100 & 90 & 80 & 70 & 60 & 50 & 40 & 30 & 20 & 10 & 0 & \mathrm{ppm}\end{array}$ 

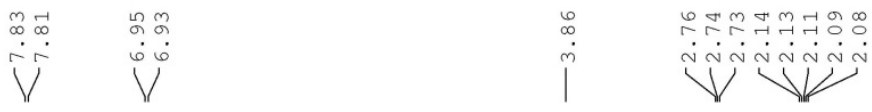<smiles>COc1ccc(C(=O)N2C(=O)CCCC2=O)cc1</smiles>

$1 e$

(400 $\mathrm{MHz}, \mathrm{CDCl}_{3}$ )

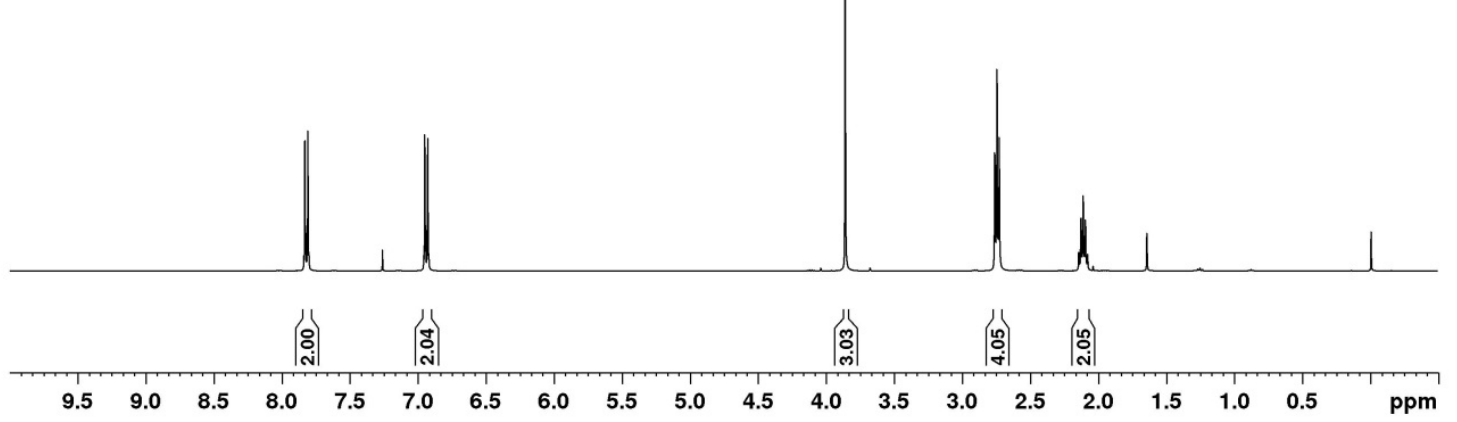

||<smiles>COc1ccc(C(=O)N2C(=O)CCCC2=O)cc1</smiles>

1e

$\left(100 \mathrm{MHz}, \mathrm{CDCl}_{3}\right)$

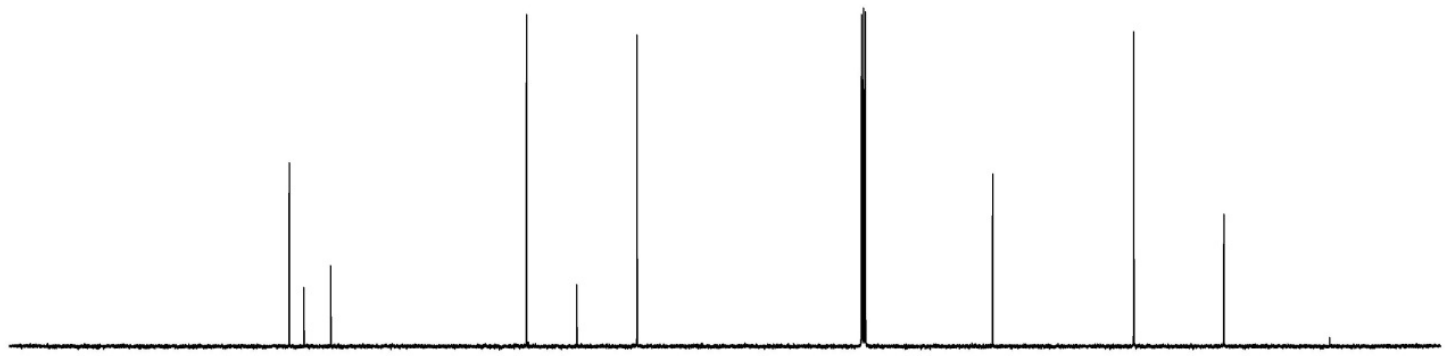

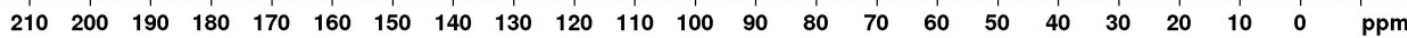



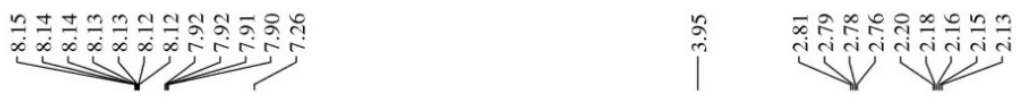

\section{i̊}<smiles>COC(=O)c1ccc(C(=O)N2C(=O)CCCC2=O)cc1</smiles>

$\left(400 \mathrm{MHz} \mathrm{CDCl}_{3}\right)$

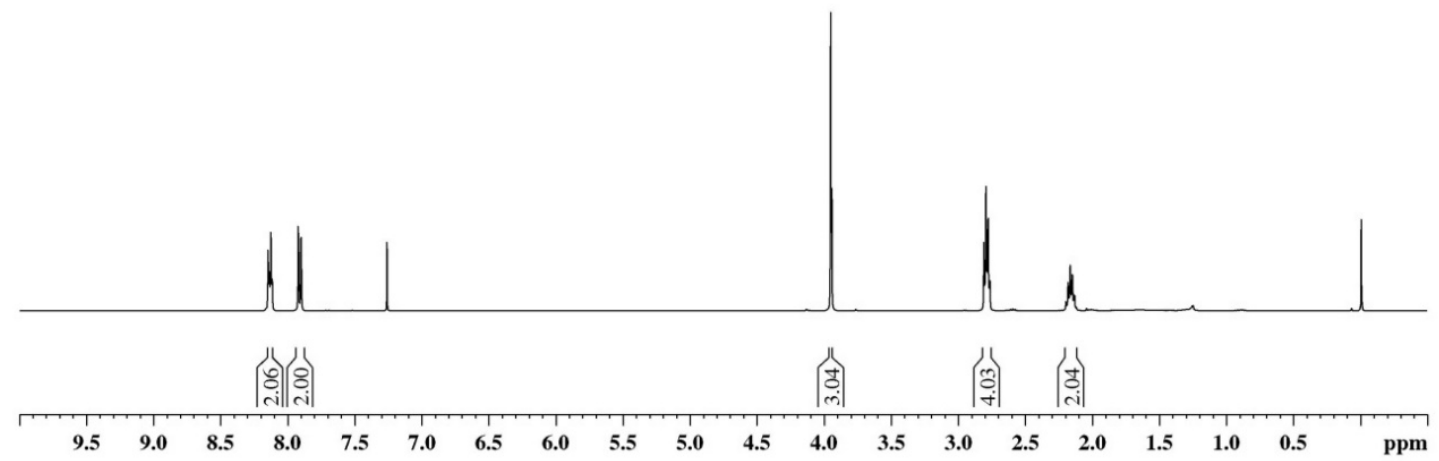<smiles>COC(=O)c1ccc(C(=O)N2C(=O)CCCC2=O)cc1</smiles>

$\left.\left(100 \mathrm{MHz}^{\mathrm{CDCl}}\right)_{3}\right)$

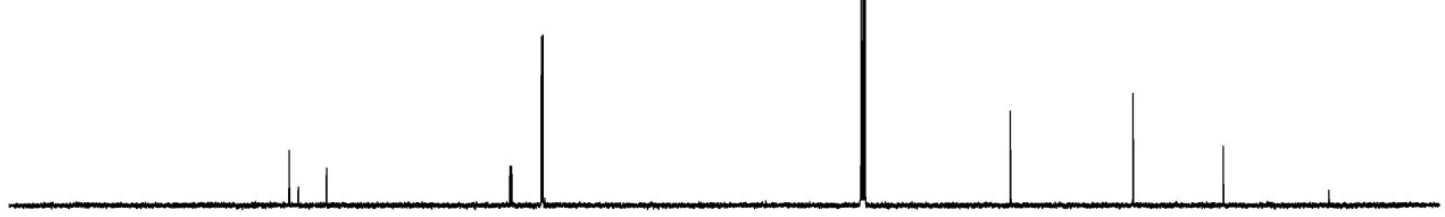

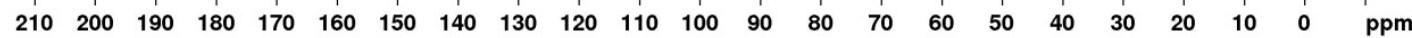




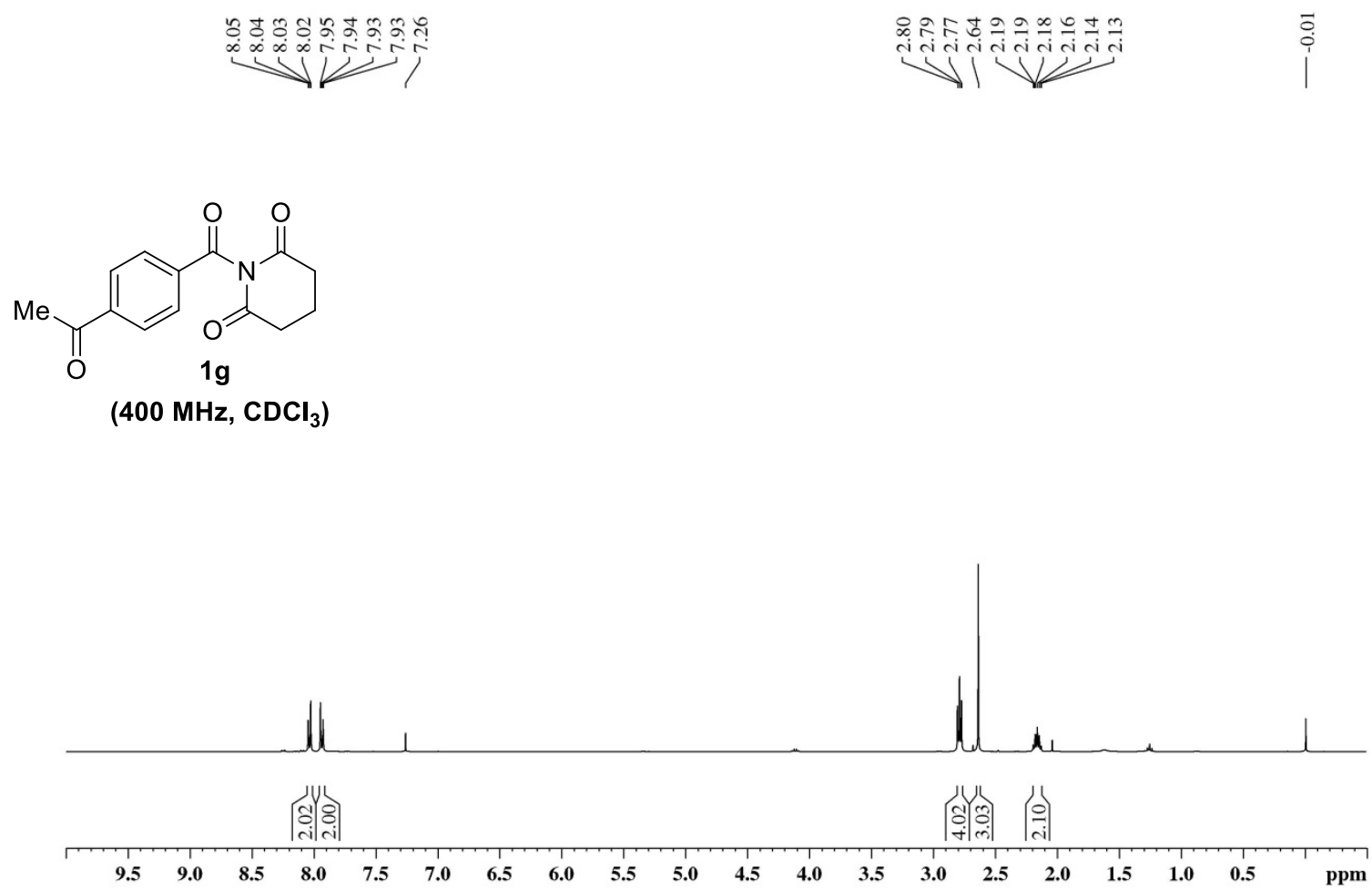

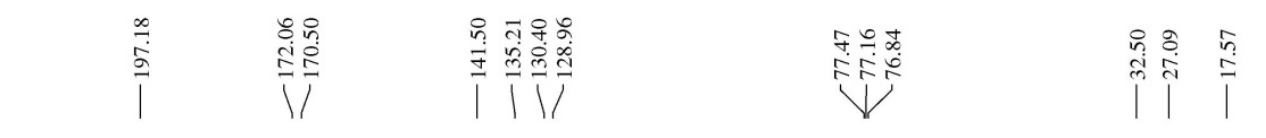<smiles>CC(=O)c1ccc(C(=O)N2C(=O)CCCC2=O)cc1</smiles>
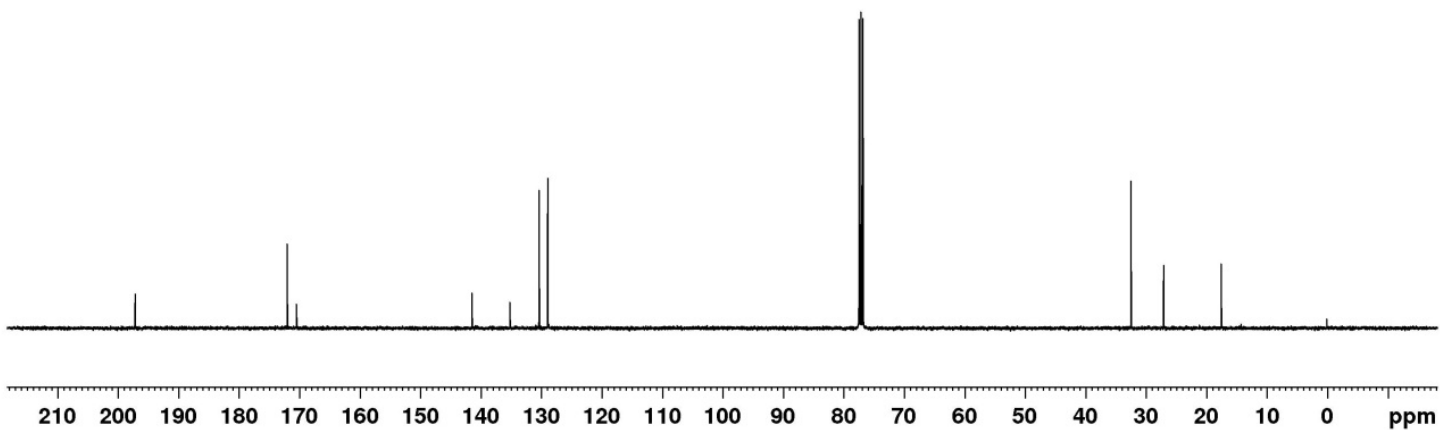

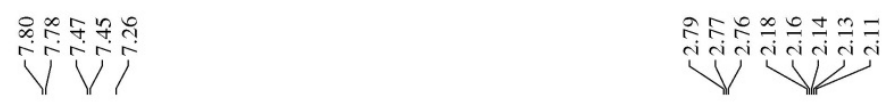<smiles>O=C1CCCC(=O)N1C(=O)c1ccc(Cl)cc1</smiles>

$1 \mathrm{~h}$

$\left(400 \mathrm{MHz} \mathrm{CDCl}_{3}\right)$

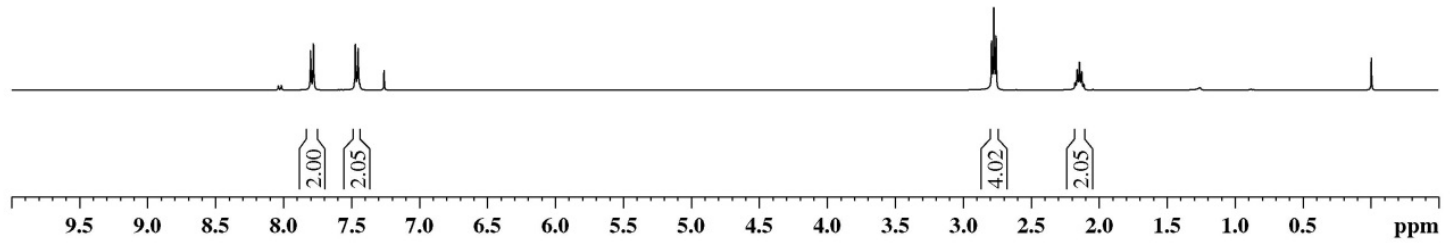

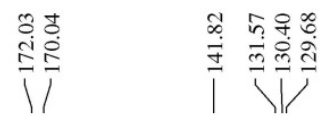

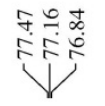<smiles>O=C1CCCC(=O)N1C(=O)c1ccc(Cl)cc1</smiles>

(100 MHz, $\mathrm{CDCl}_{3}$ )

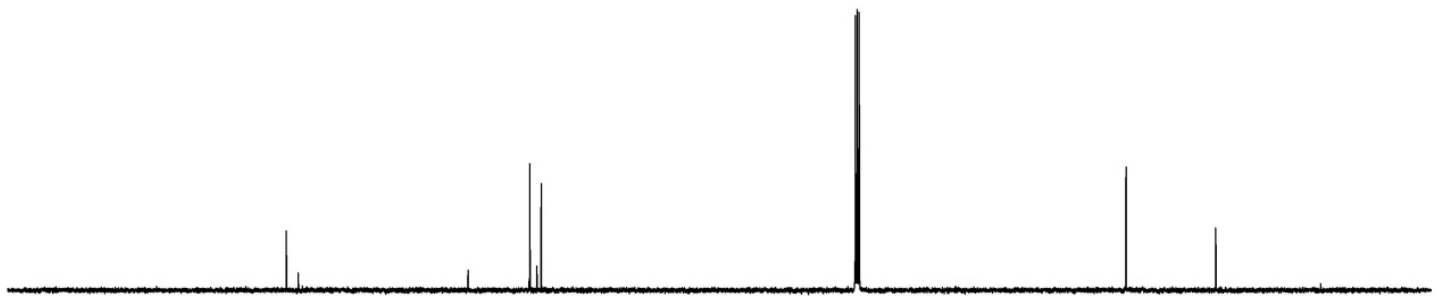

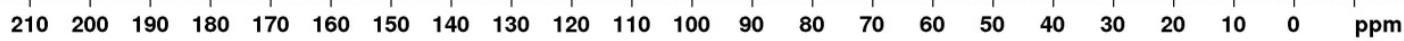



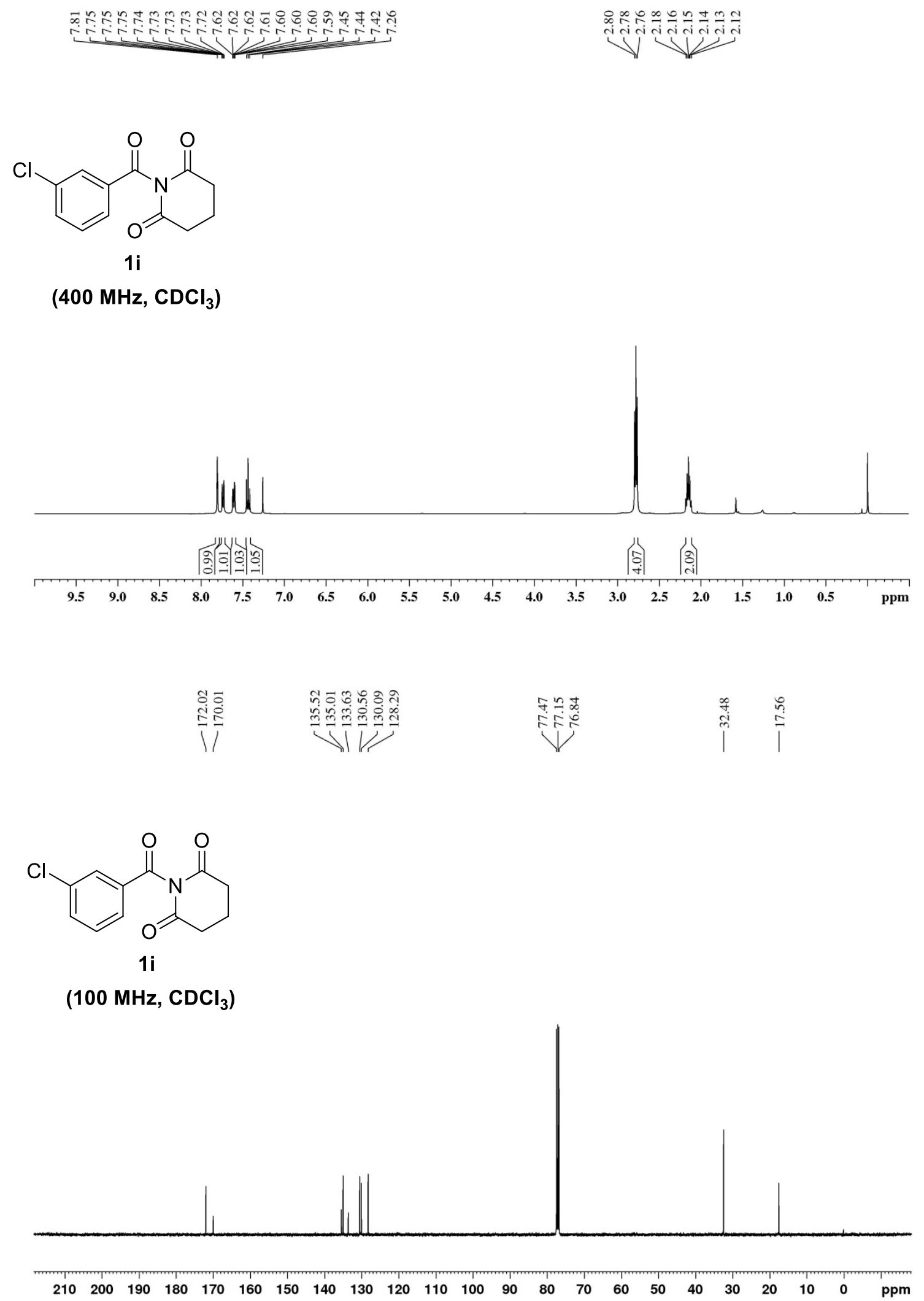

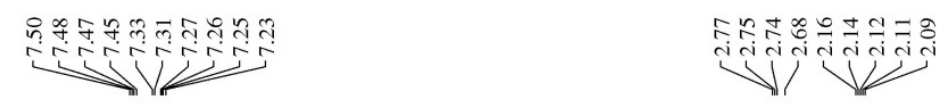

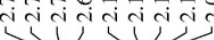<smiles>Cc1ccccc1C(=O)N1C(=O)CCCC1=O</smiles>

1j

(400 $\mathrm{MHz}, \mathrm{CDCl}_{3}$ )

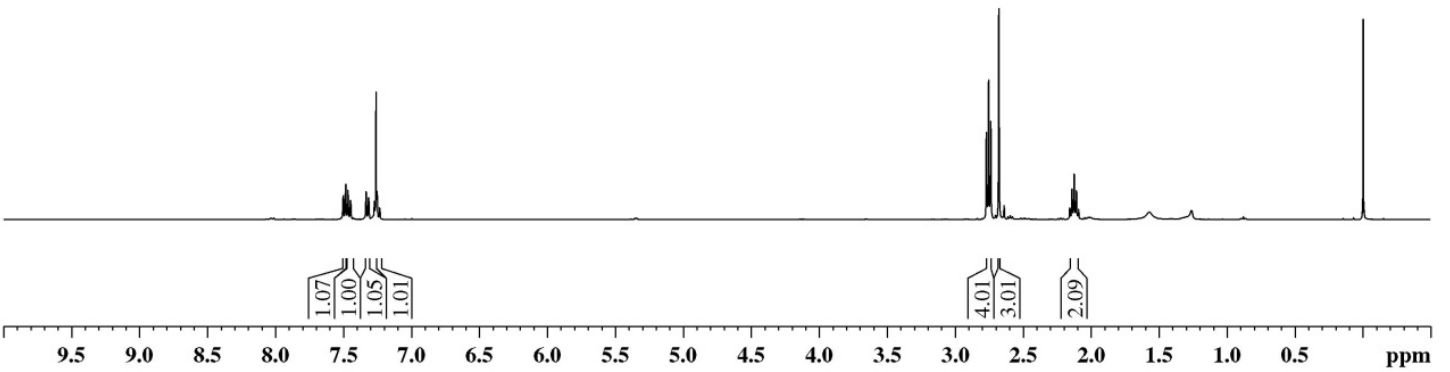

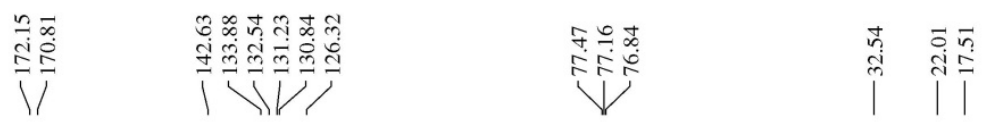<smiles>Cc1ccccc1C(=O)N1C(=O)CCCC1=O</smiles>

1j

$\left(100 \mathrm{MHz} \mathrm{CDCl}_{3}\right)$

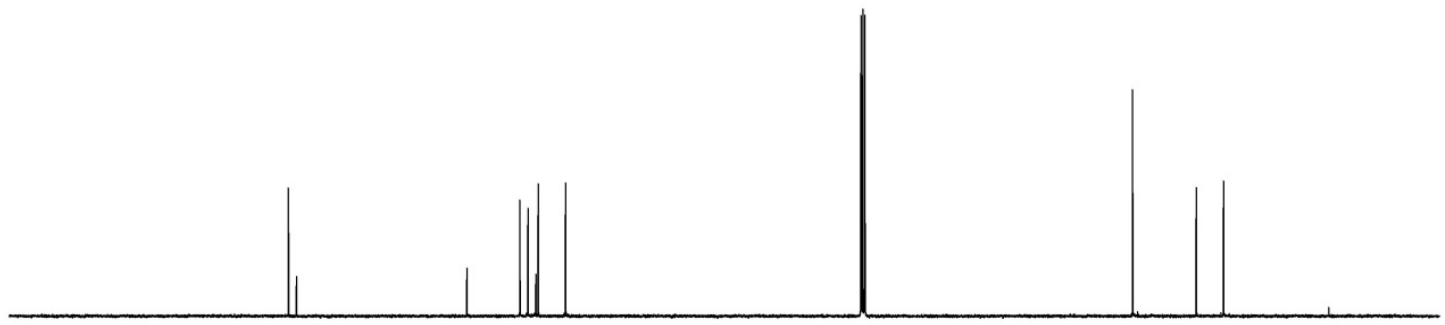

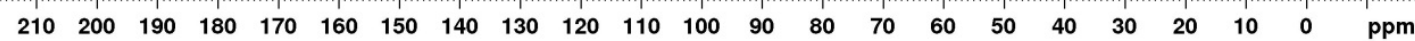



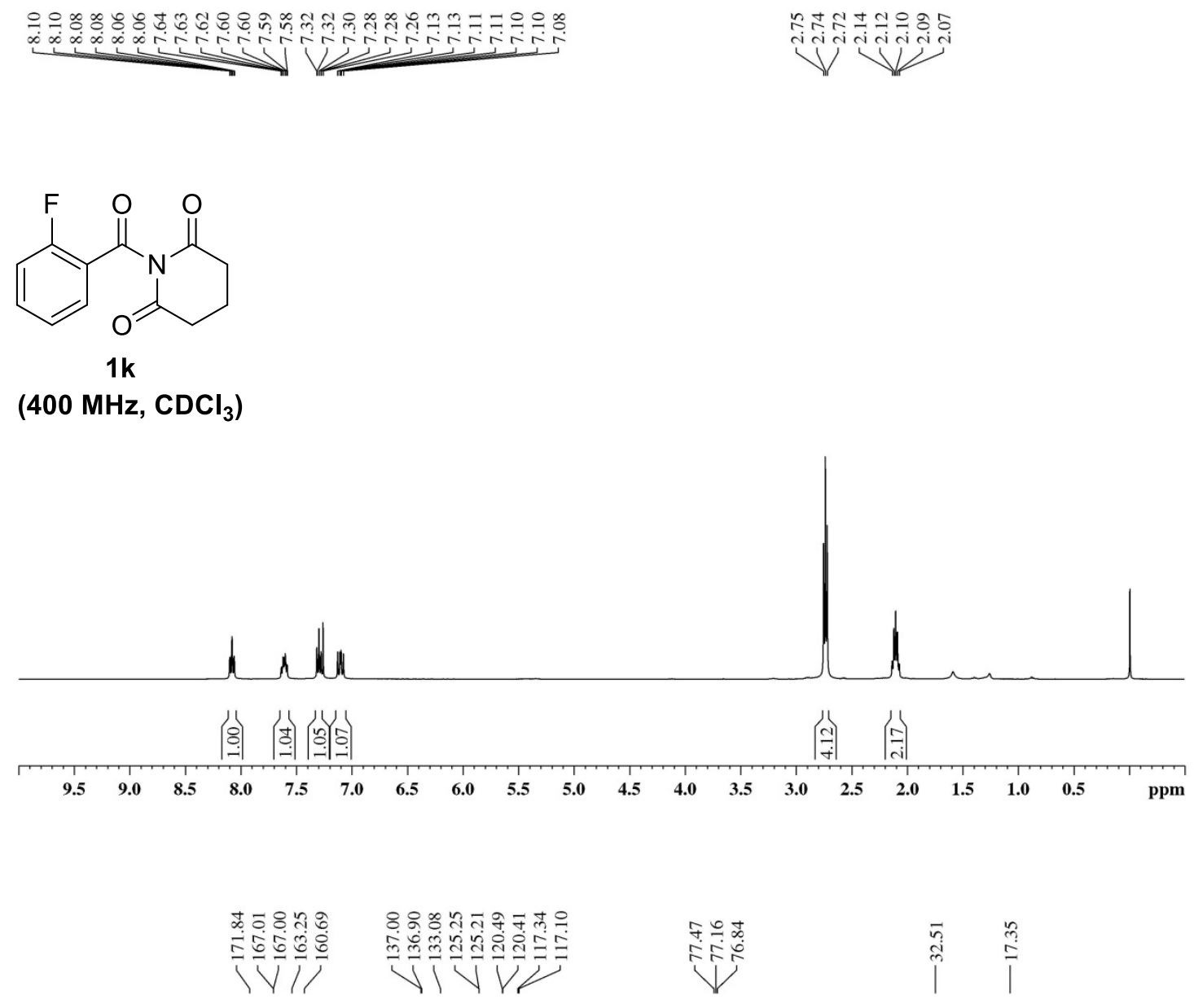<smiles>O=C1CCCC(=O)N1C(=O)c1ccccc1F</smiles>

$1 \mathrm{k}$

$\left(100 \mathrm{MHz}, \mathrm{CDCl}_{3}\right)$

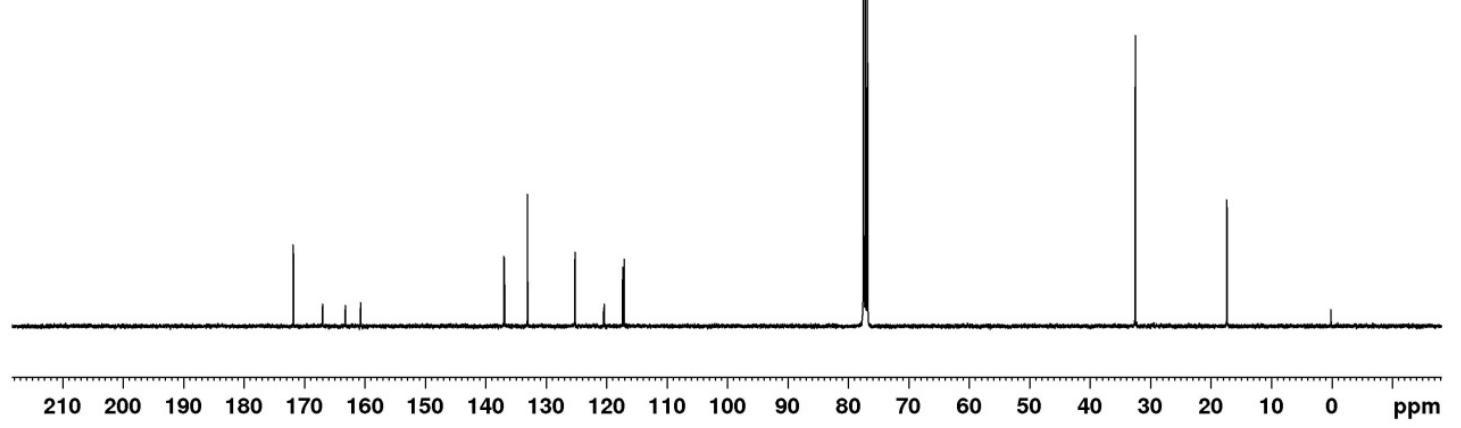




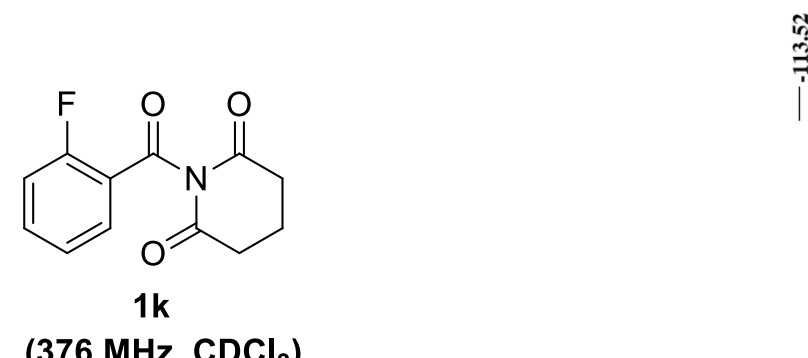

ติ

(376 MHz, $\mathrm{CDCl}_{3}$ )

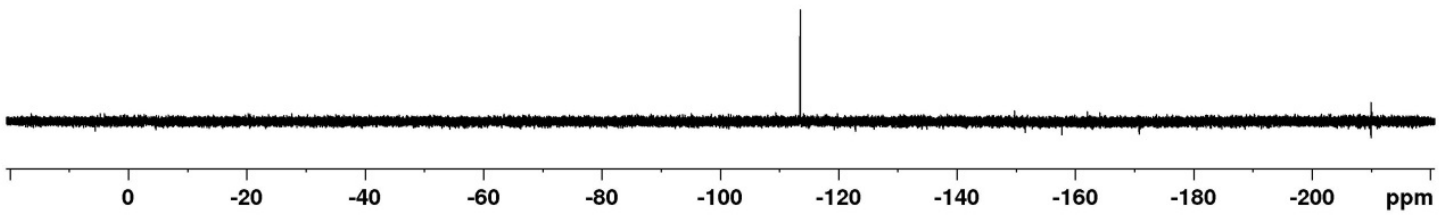



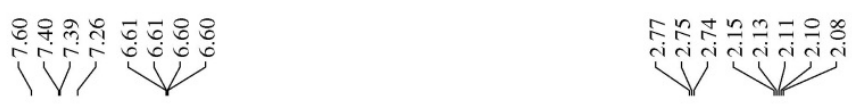<smiles>O=C1CCCC(=O)N1C(=O)c1ccco1</smiles>

11

(400 $\mathrm{MHz}, \mathrm{CDCl}_{3}$ )

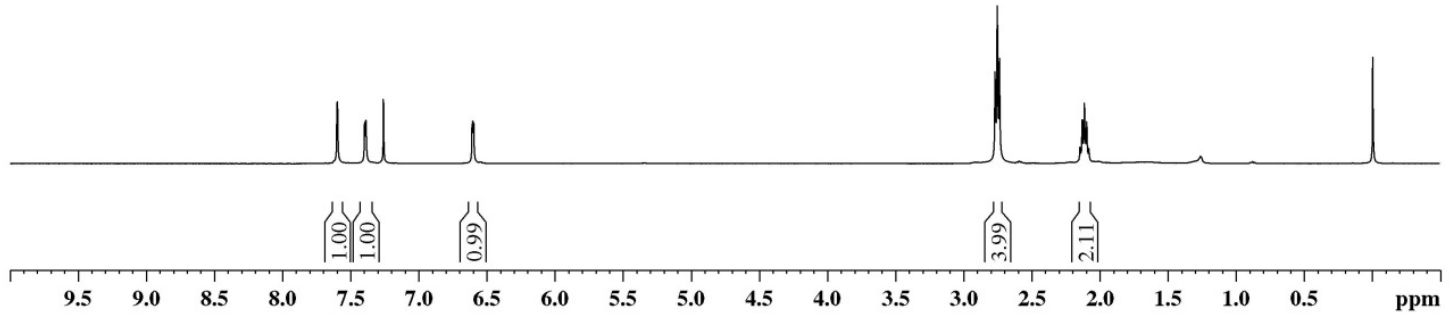

†<smiles>O=C1CCCC(=O)N1C(=O)c1ccco1</smiles>

1 I

(100 $\left.\mathrm{MHz} \mathrm{CDCl}_{3}\right)$

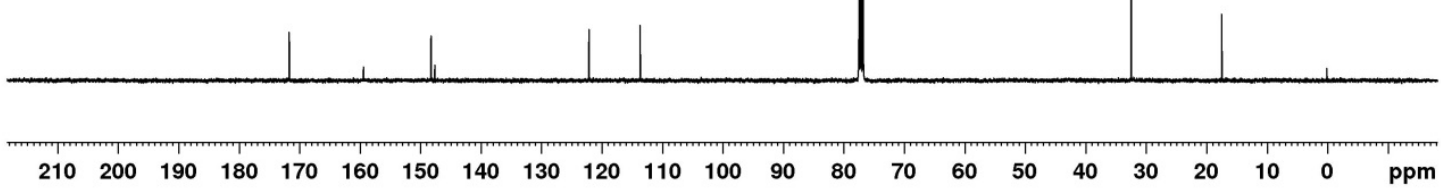



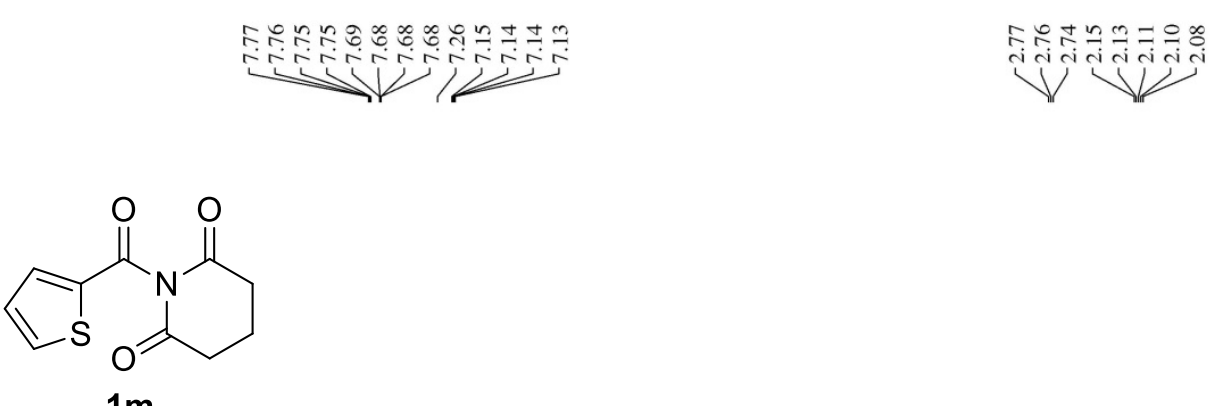

(400 MHz, $\mathrm{CDCl}_{3}$ )
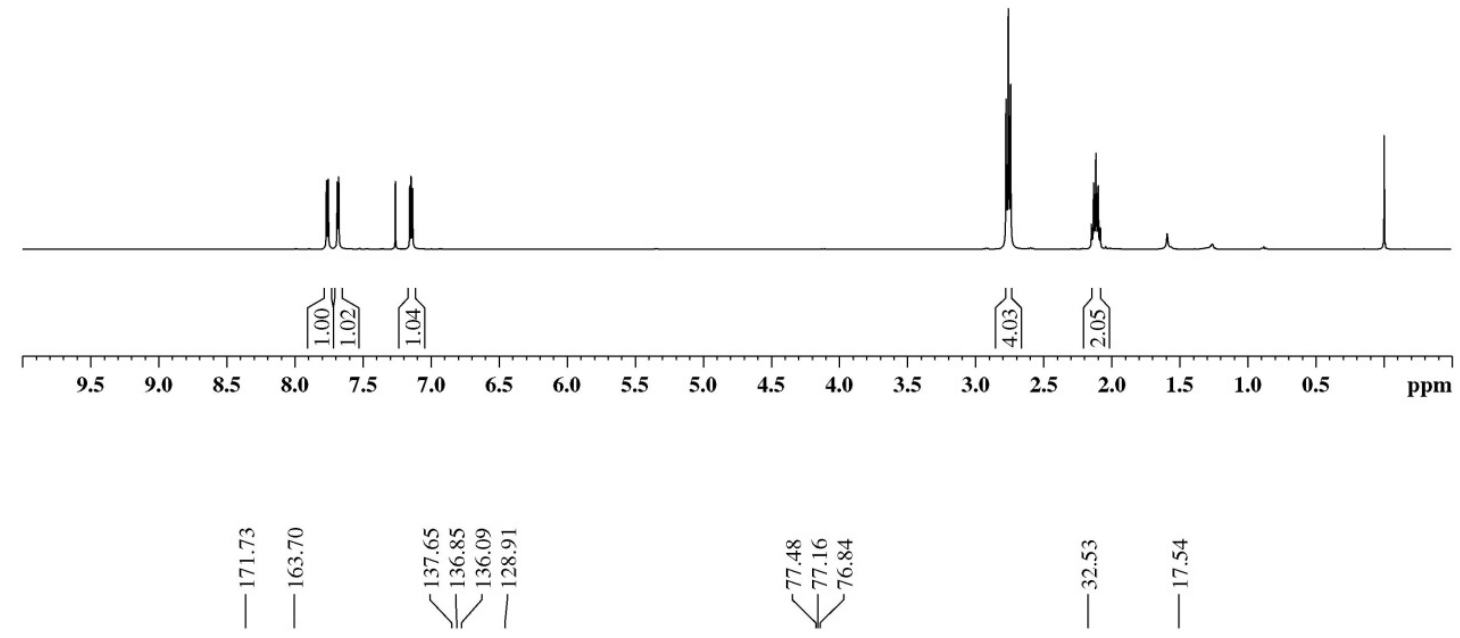<smiles>O=C1CCCC(=O)N1C(=O)c1cccs1</smiles>

$1 \mathrm{~m}$

(100 MHz, $\mathrm{CDCl}_{3}$ )

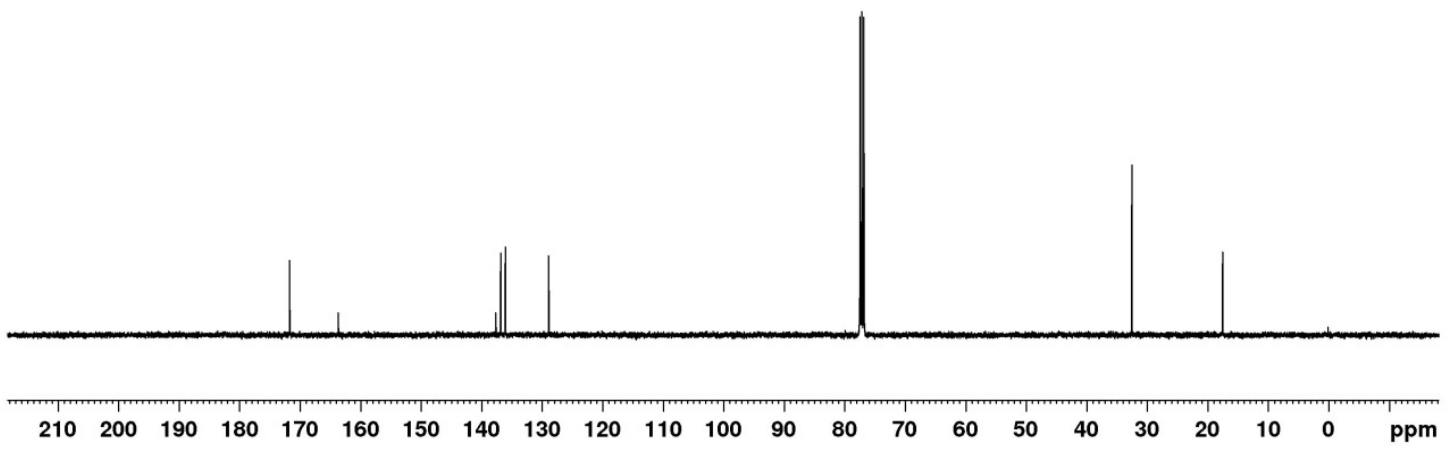




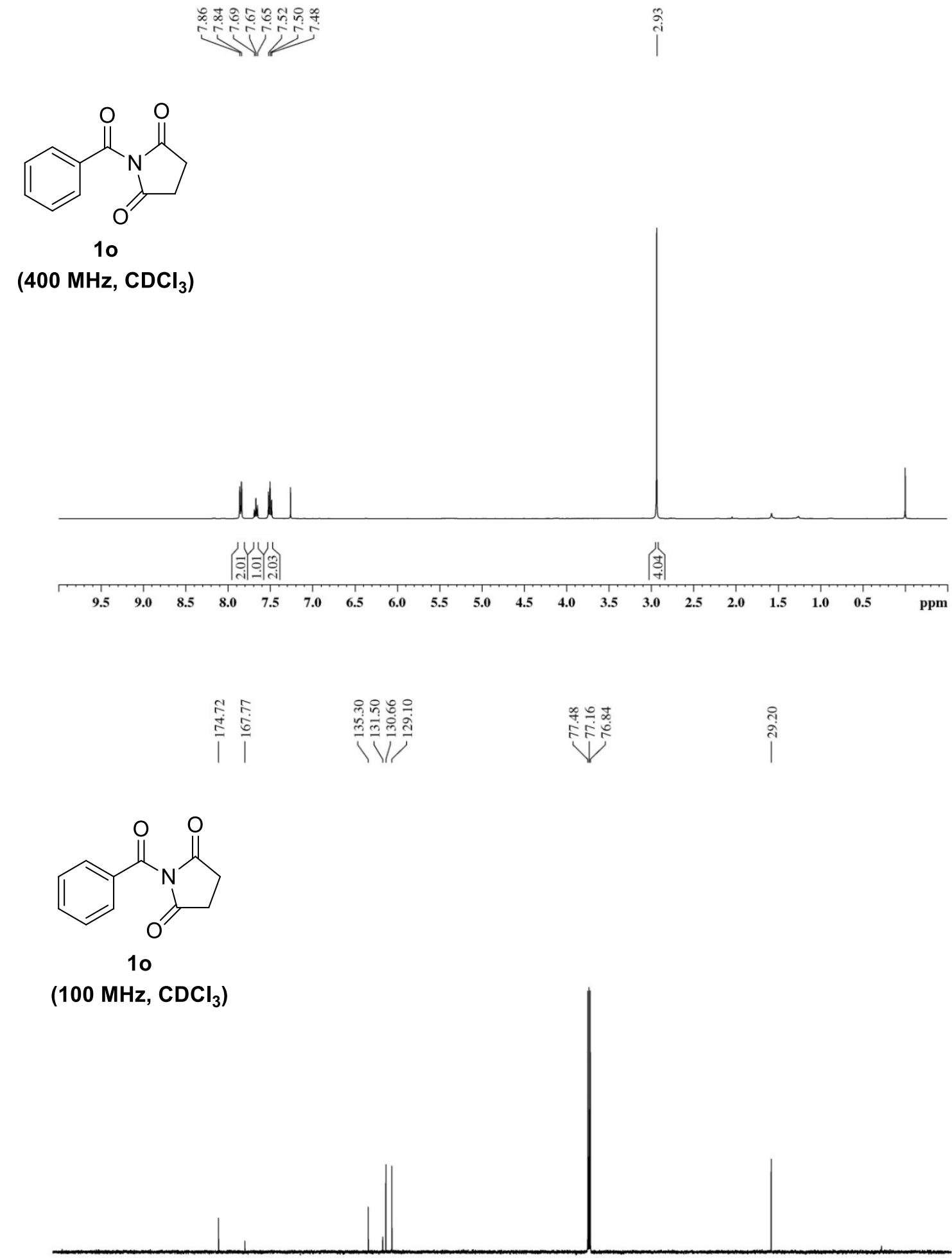

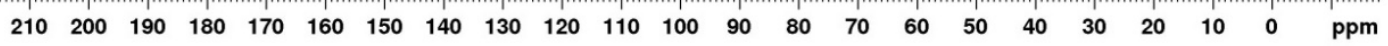




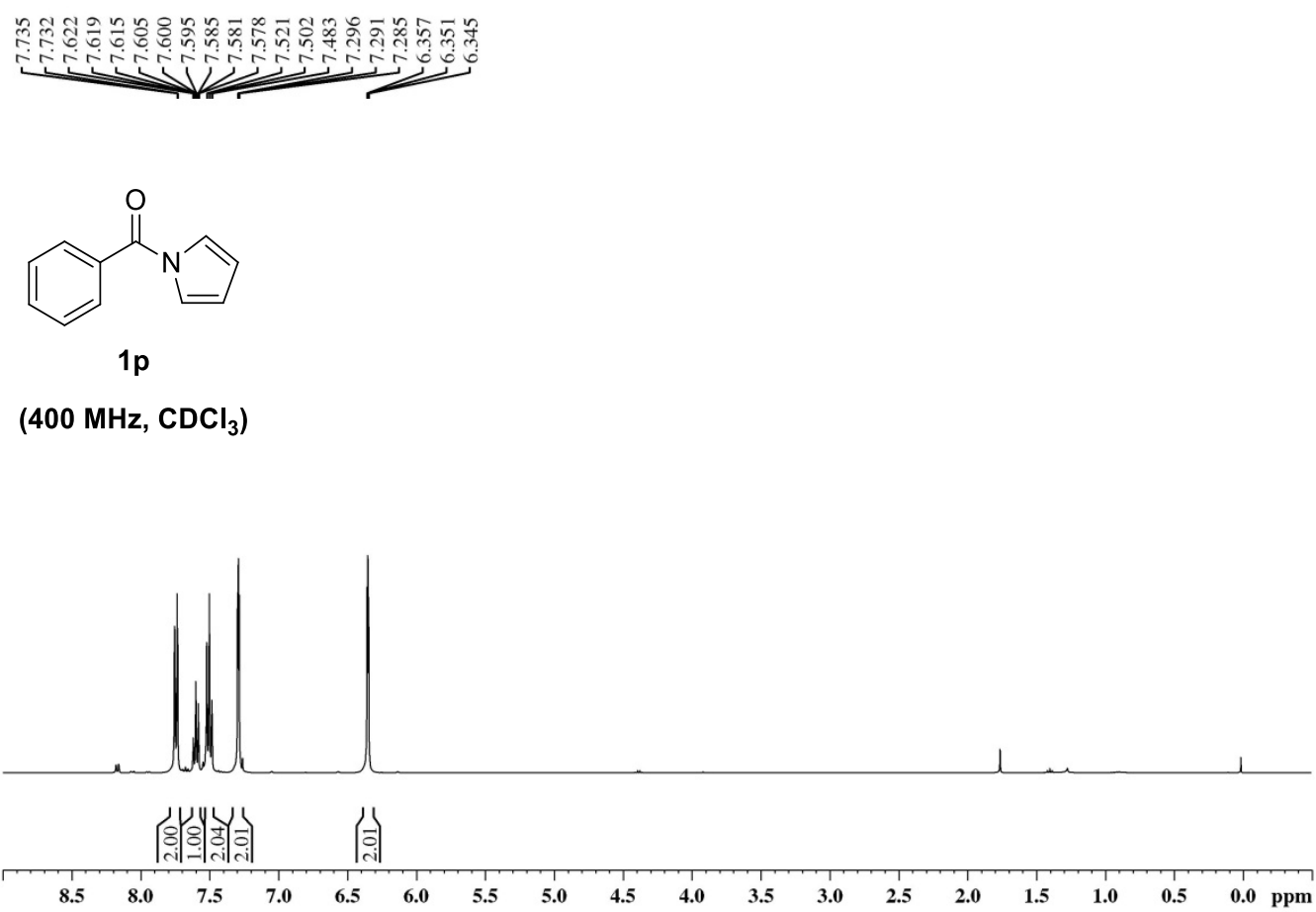

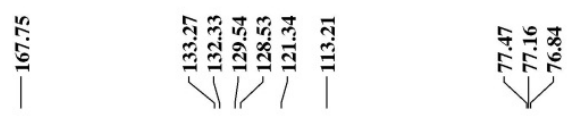<smiles>O=C(c1ccccc1)n1cccc1</smiles>

$1 \mathrm{p}$

$\left(100 \mathrm{MHz}, \mathrm{CDCl}_{3}\right)$

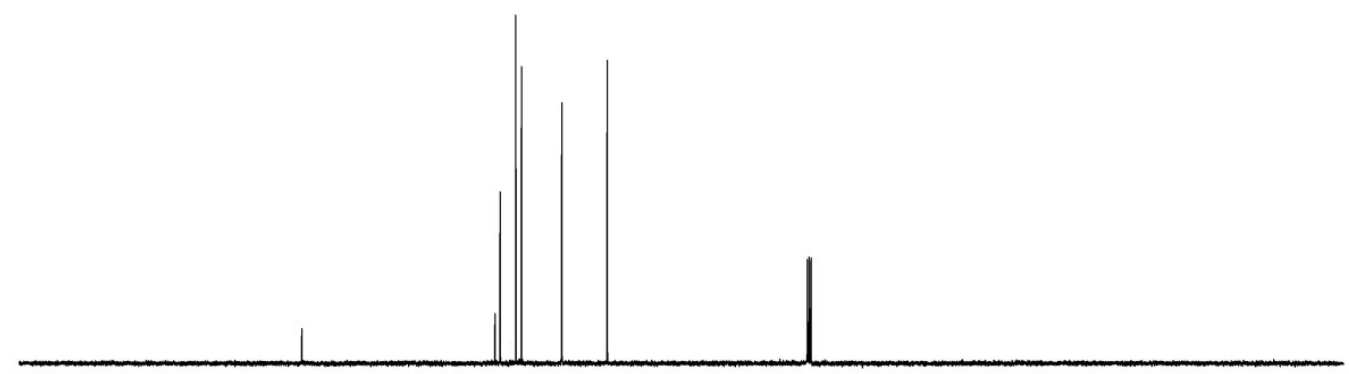

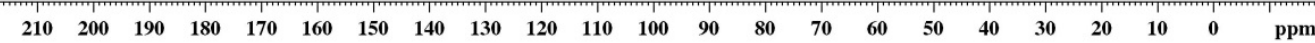




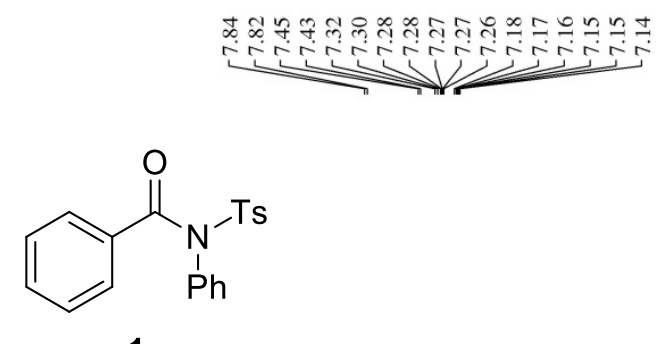

19

(400 MHz, $\mathrm{CDCl}_{3}$ )
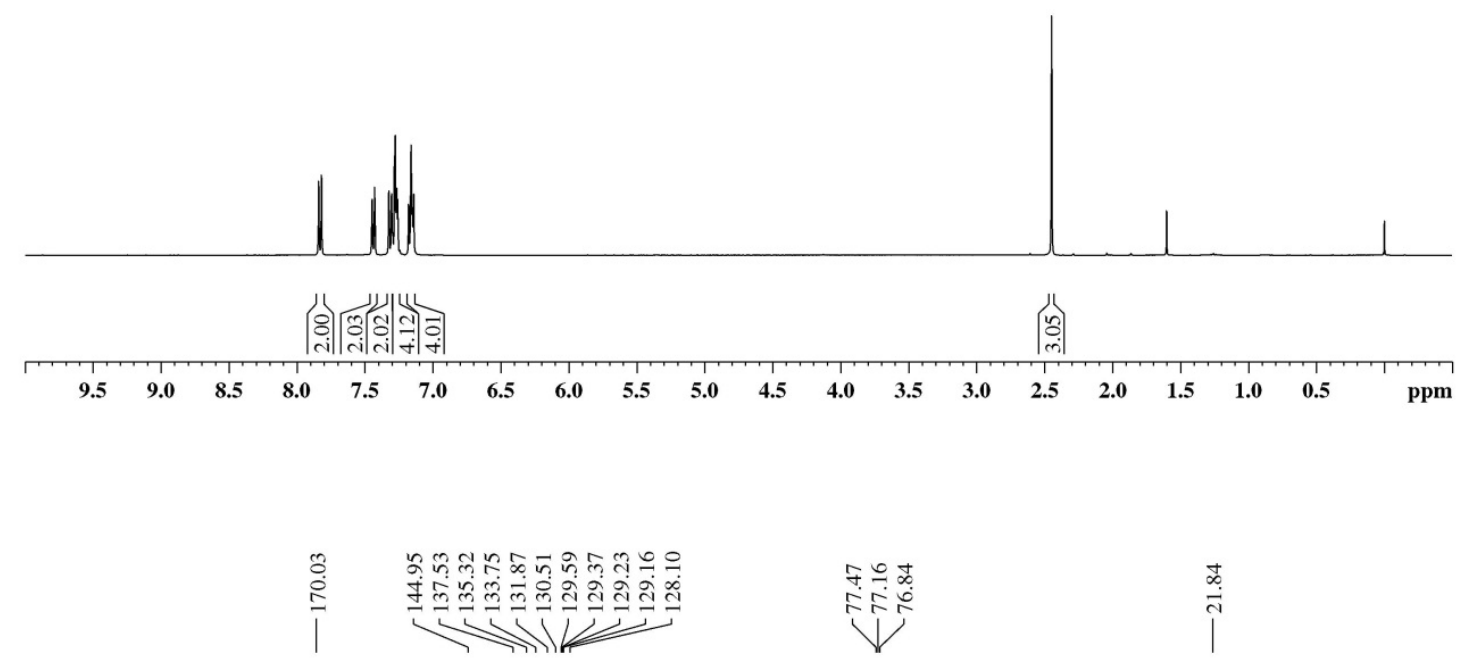<smiles>O=C(c1ccccc1)N([I-])c1ccccc1</smiles>

19

(100 MHz, $\mathrm{CDCl}_{3}$ )

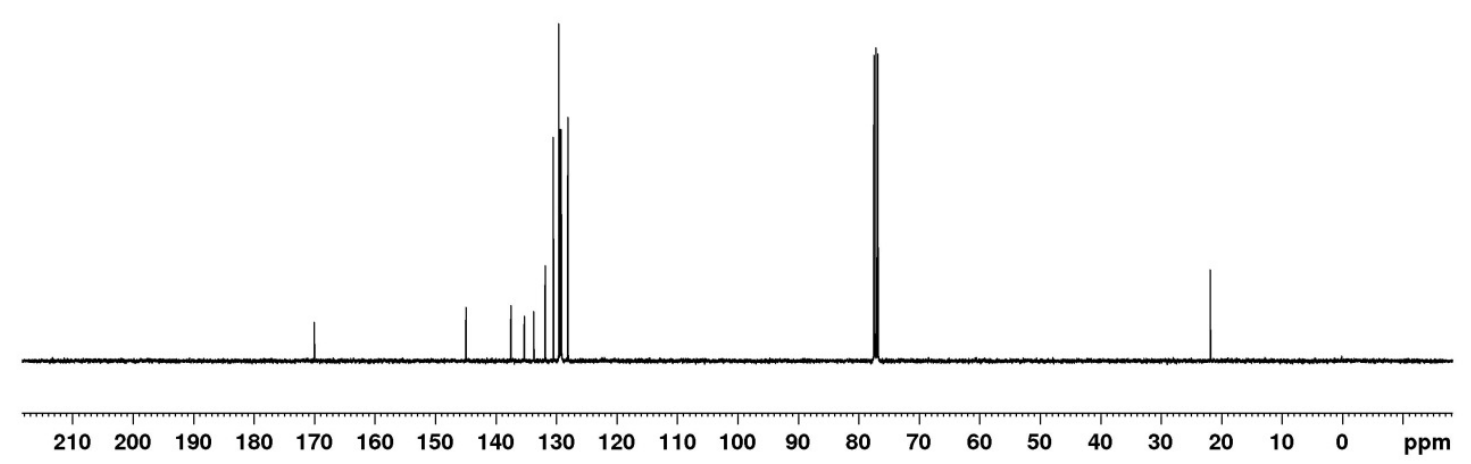


<smiles>CC(C)(C)N(C(=O)c1ccccc1)c1ccccc1</smiles>

$1 \mathrm{r}$

$\left(400 \mathrm{MHz}, \mathrm{CDCl}_{3}\right)$
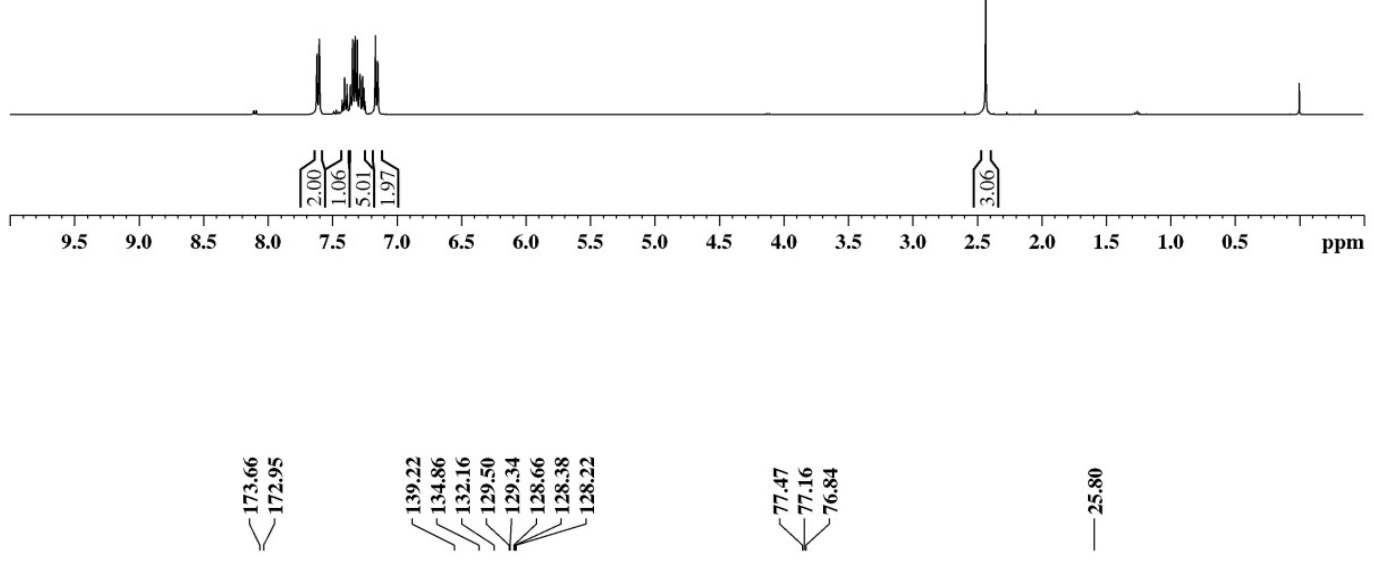<smiles>CC(C)(C)N(C(=O)c1ccccc1)c1ccccc1</smiles>

$\left(100 \mathrm{MHz} \mathrm{CDCl}_{3}\right)$

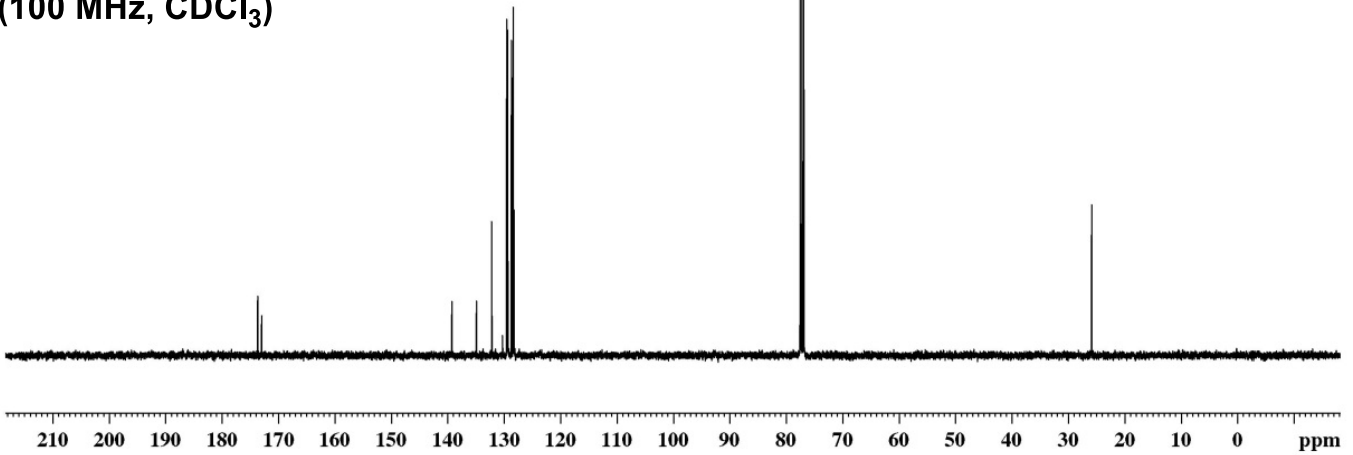



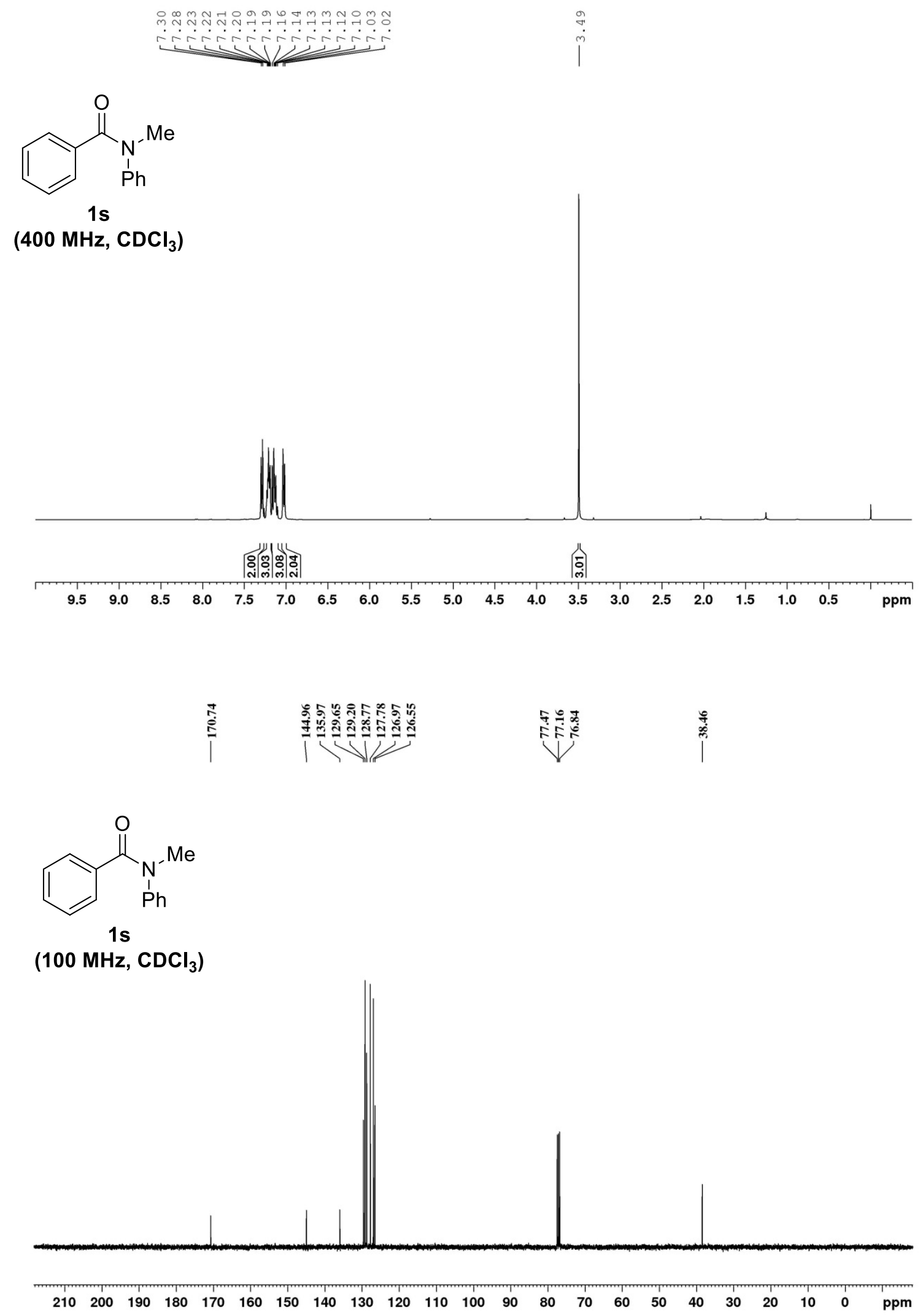

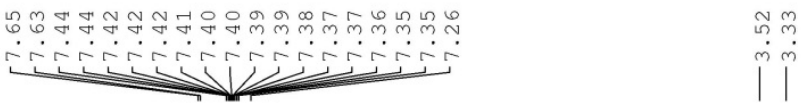

$\overbrace{\substack{1 \\ \mathrm{Me}}}^{\mathrm{O}}$

$1 \mathrm{t}$

(400 MHz, $\mathrm{CDCl}_{3}$ )
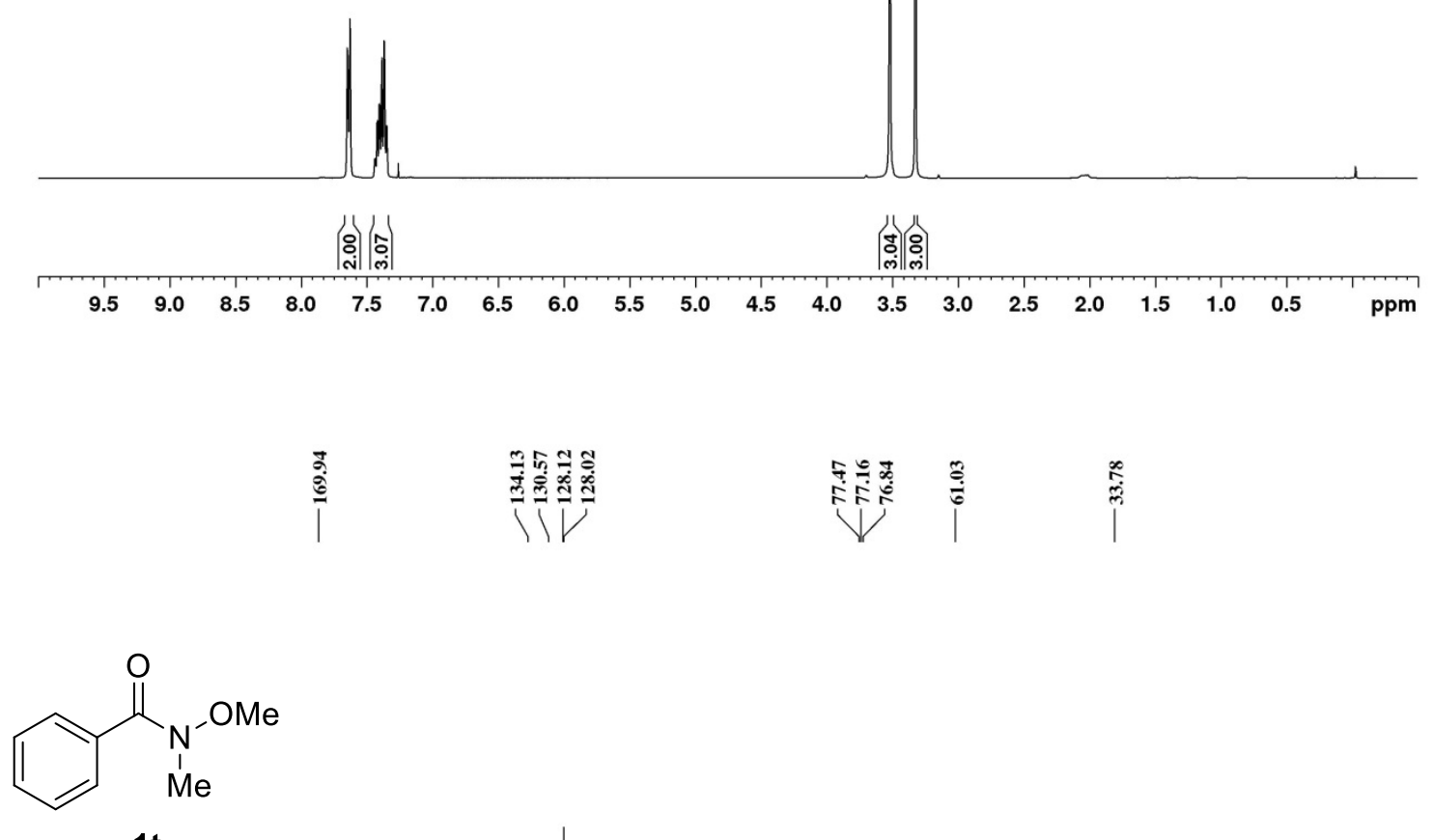

$1 \mathrm{t}$

$\left(100 \mathrm{MHz} \mathrm{CDCl}_{3}\right)$

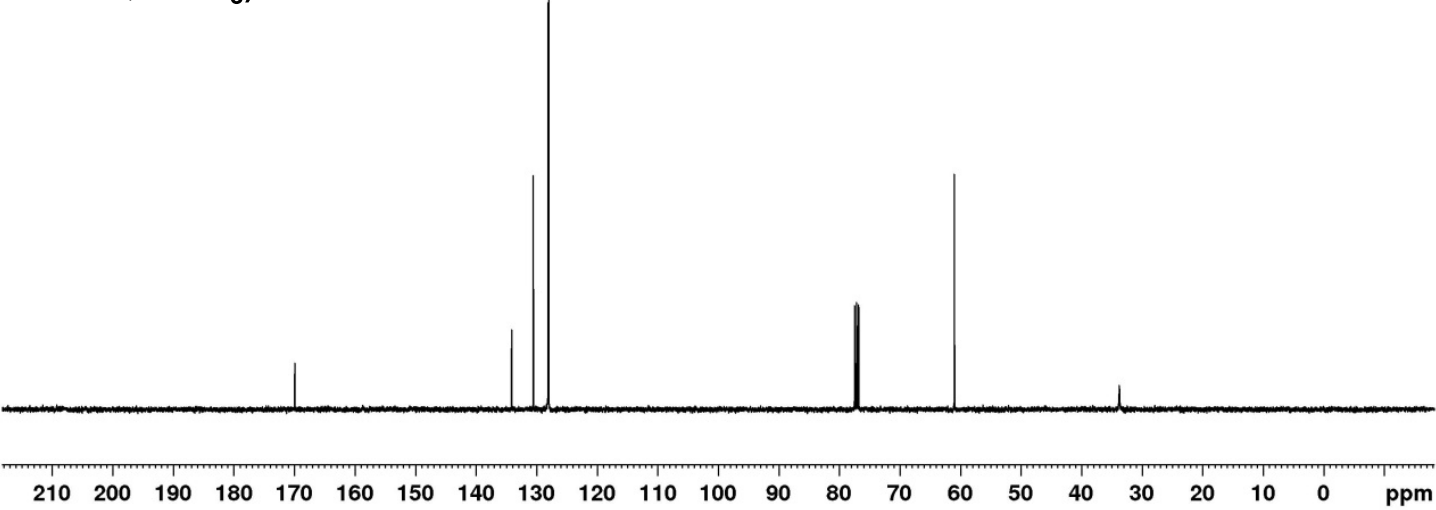




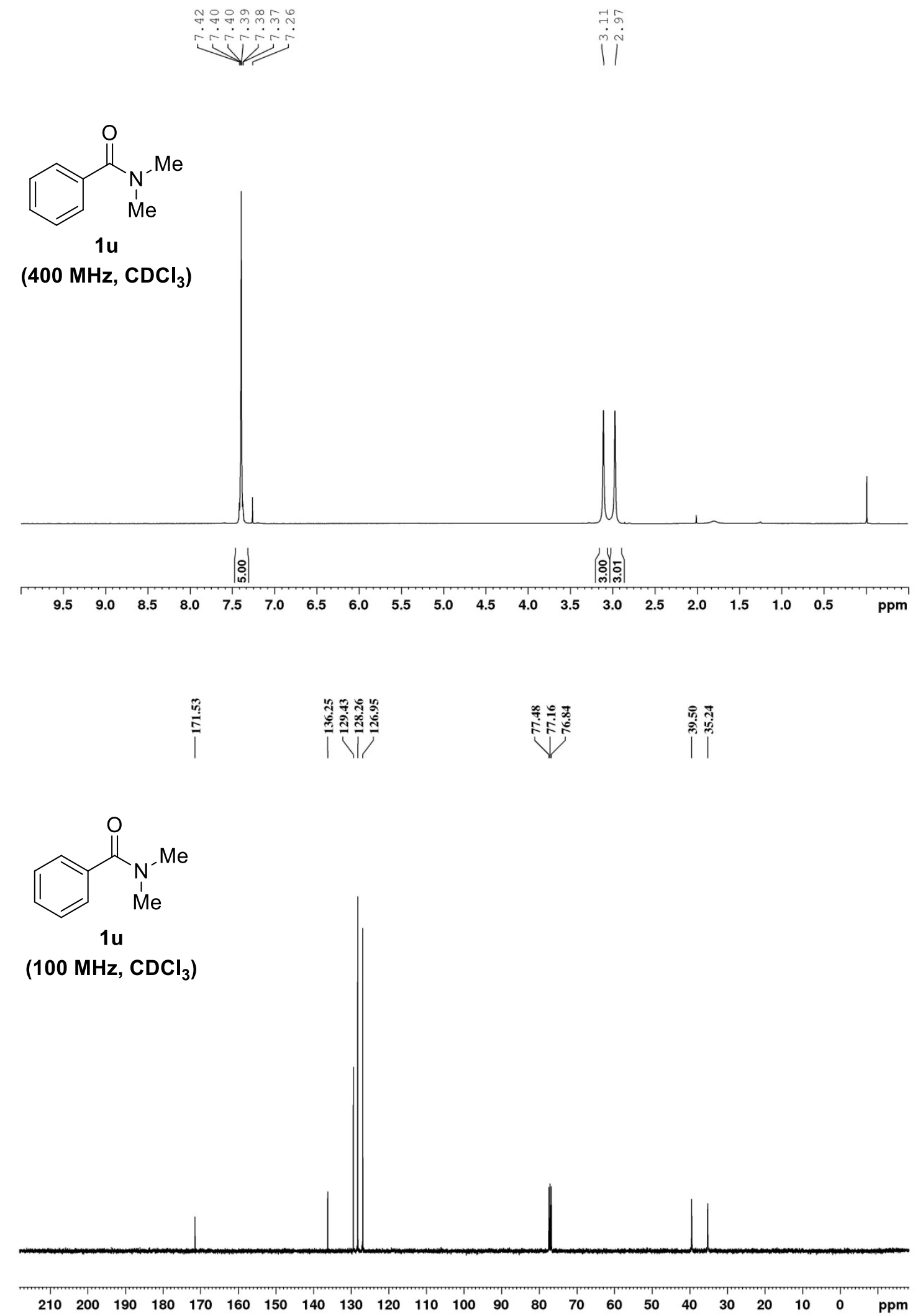



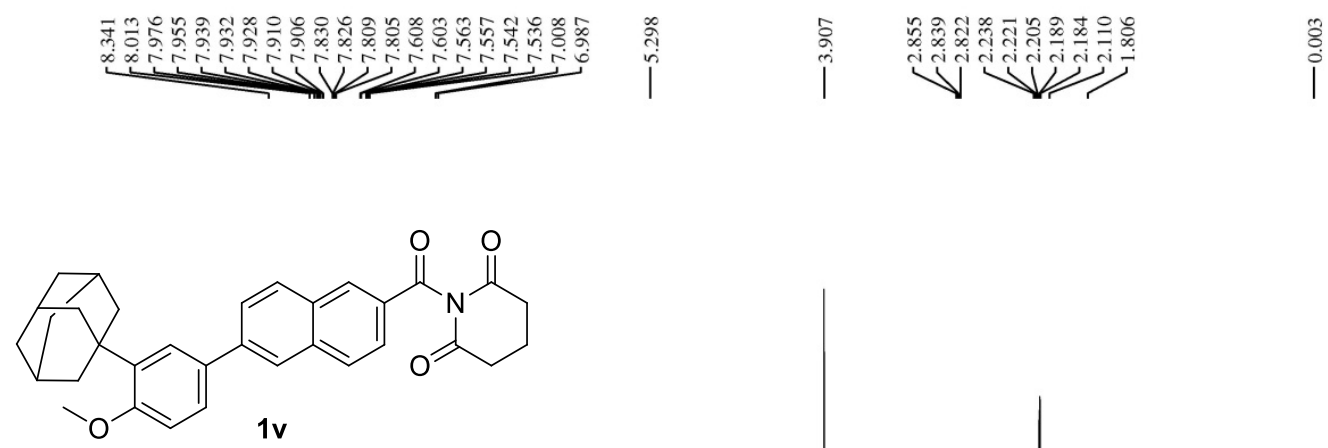

(400 MHz, $\mathrm{CDCl}_{3}$ )
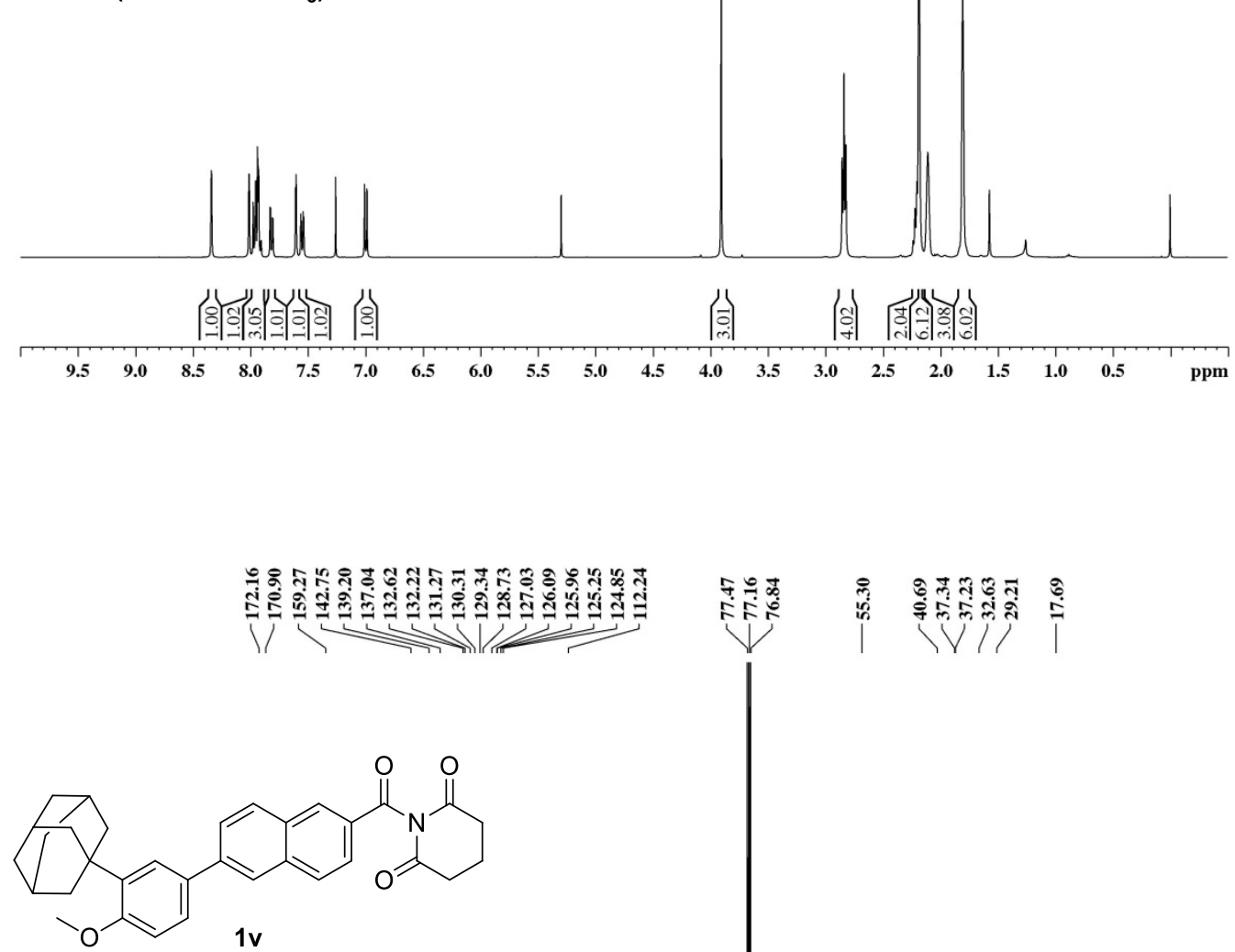

$\left(100 \mathrm{MHz}, \mathrm{CDCl}_{3}\right)$
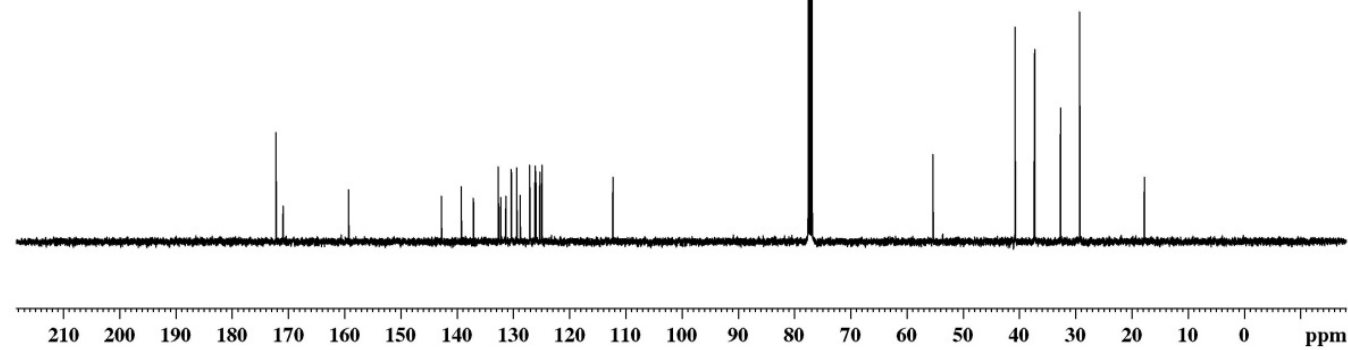

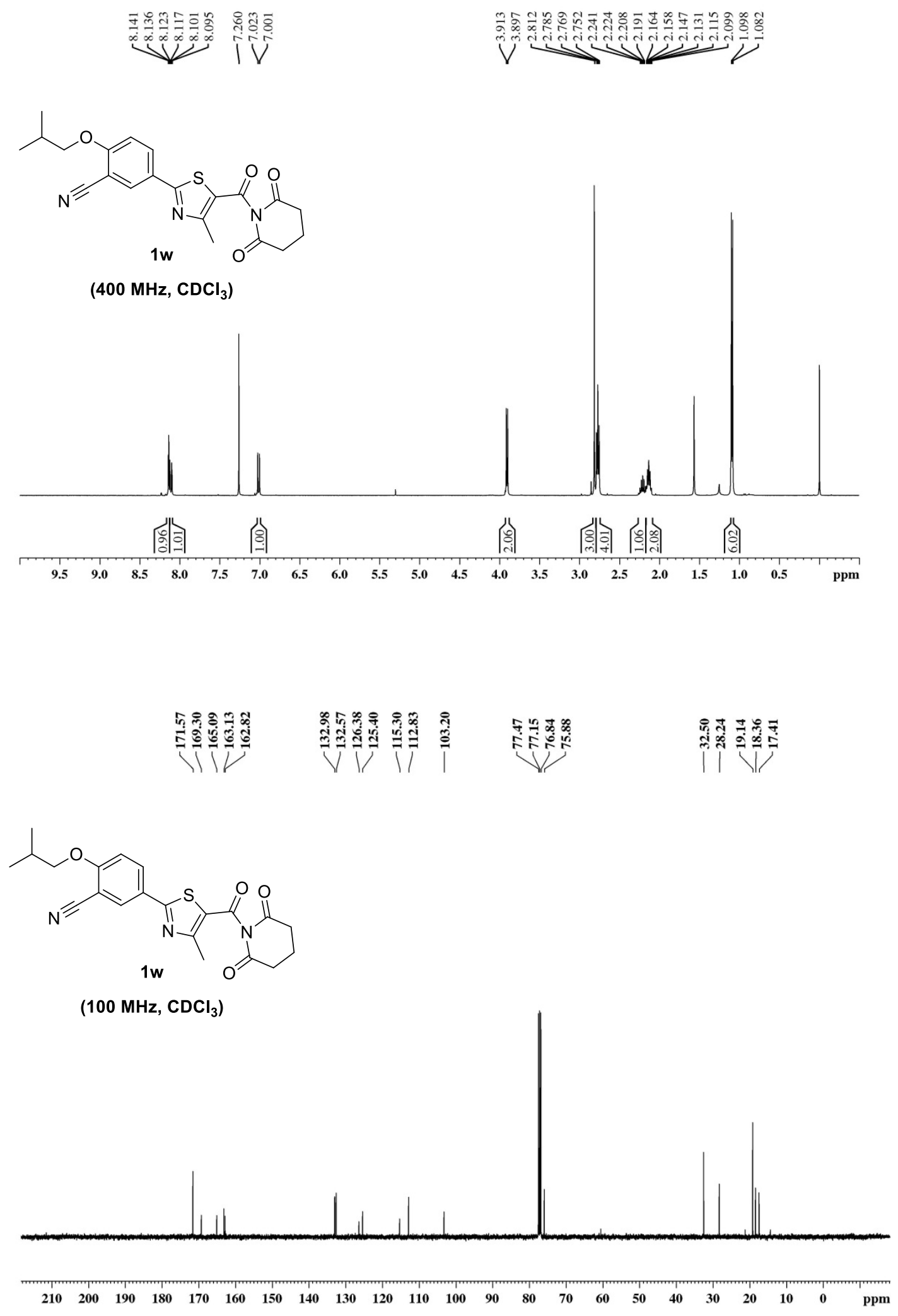


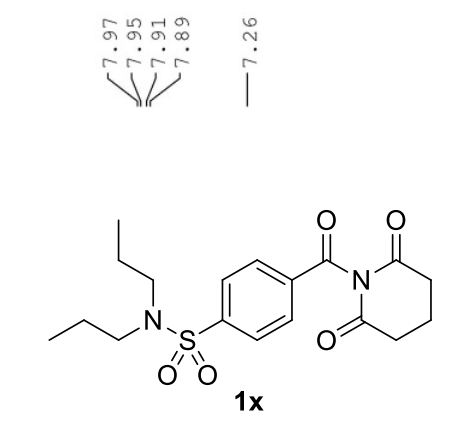

$\left(400 \mathrm{MHz}, \mathrm{CDCl}_{3}\right)$
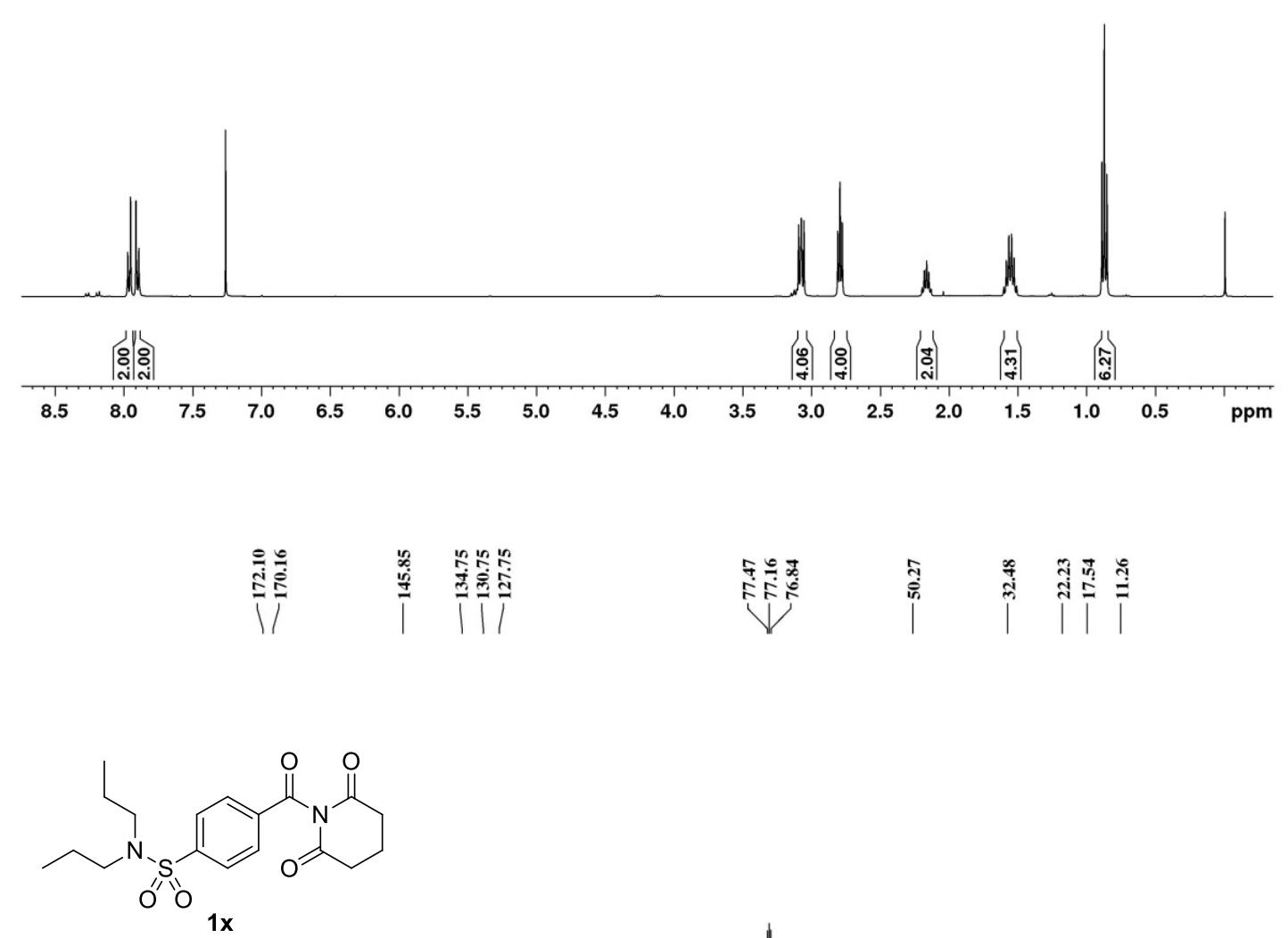

$\left(100 \mathrm{MHz}, \mathrm{CDCl}_{3}\right)$
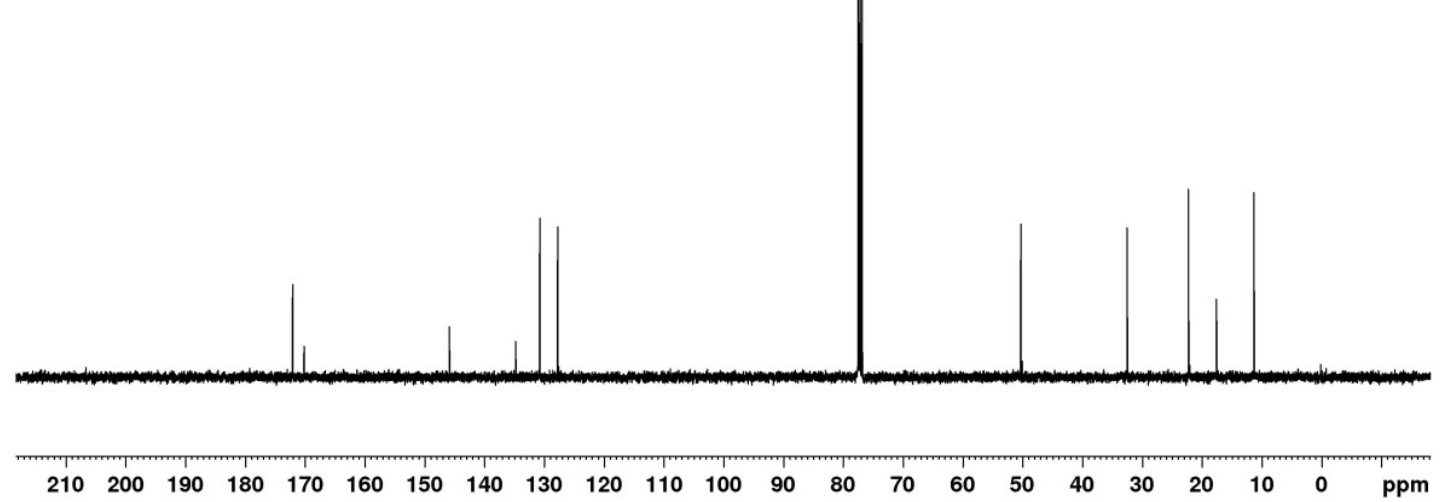

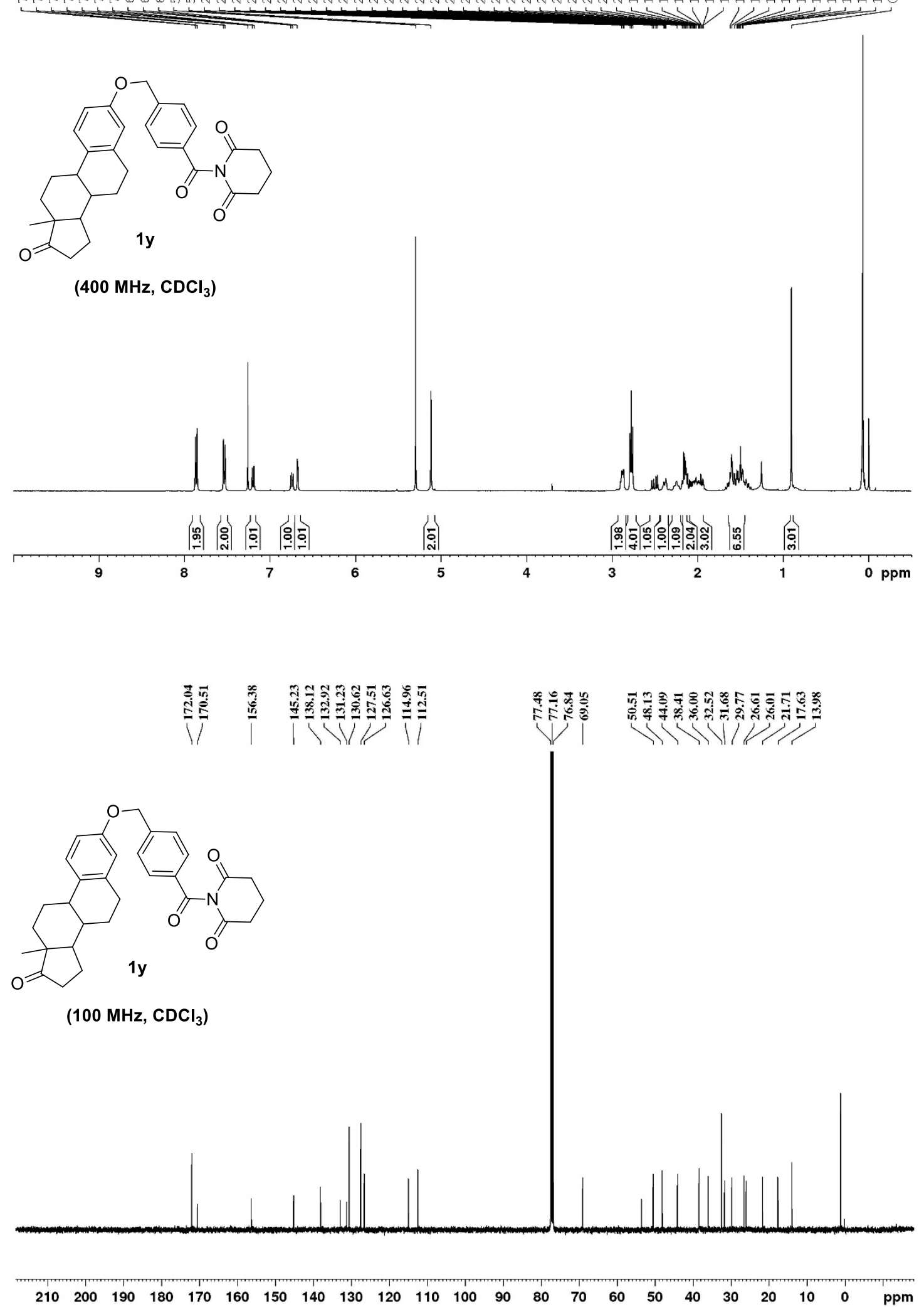


\section{${ }^{1} \mathrm{H},{ }^{13} \mathrm{C}$ and ${ }^{19} \mathrm{~F}$ NMR of Thioesters}
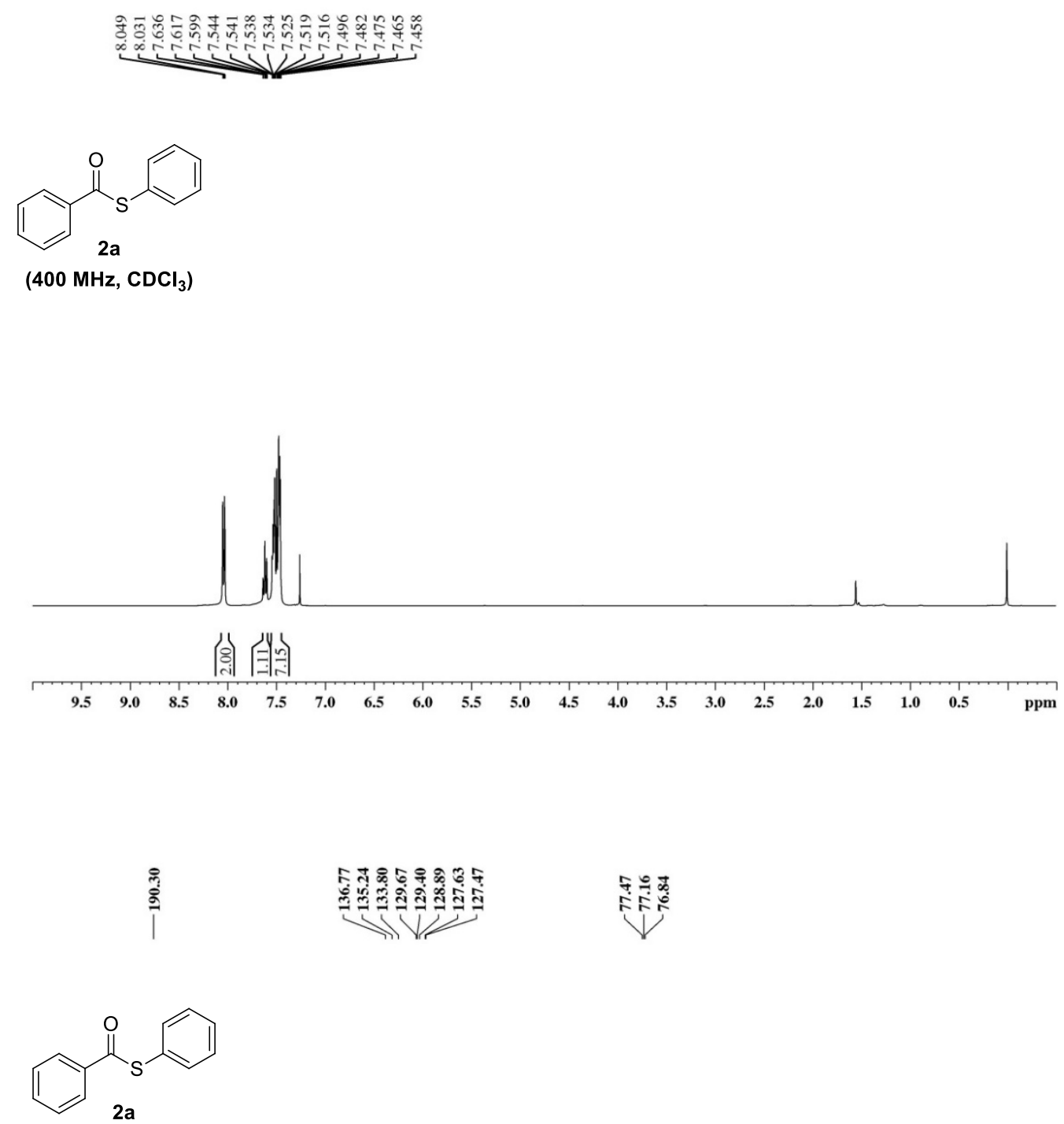

$\left(100 \mathrm{MHz} \mathrm{CDCl}_{3}\right)$
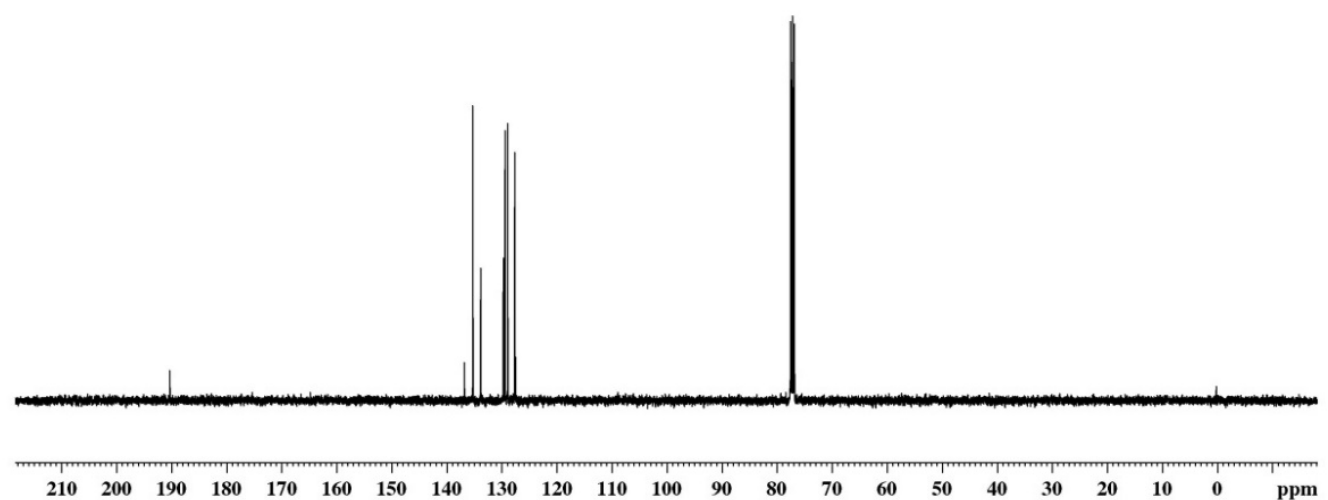

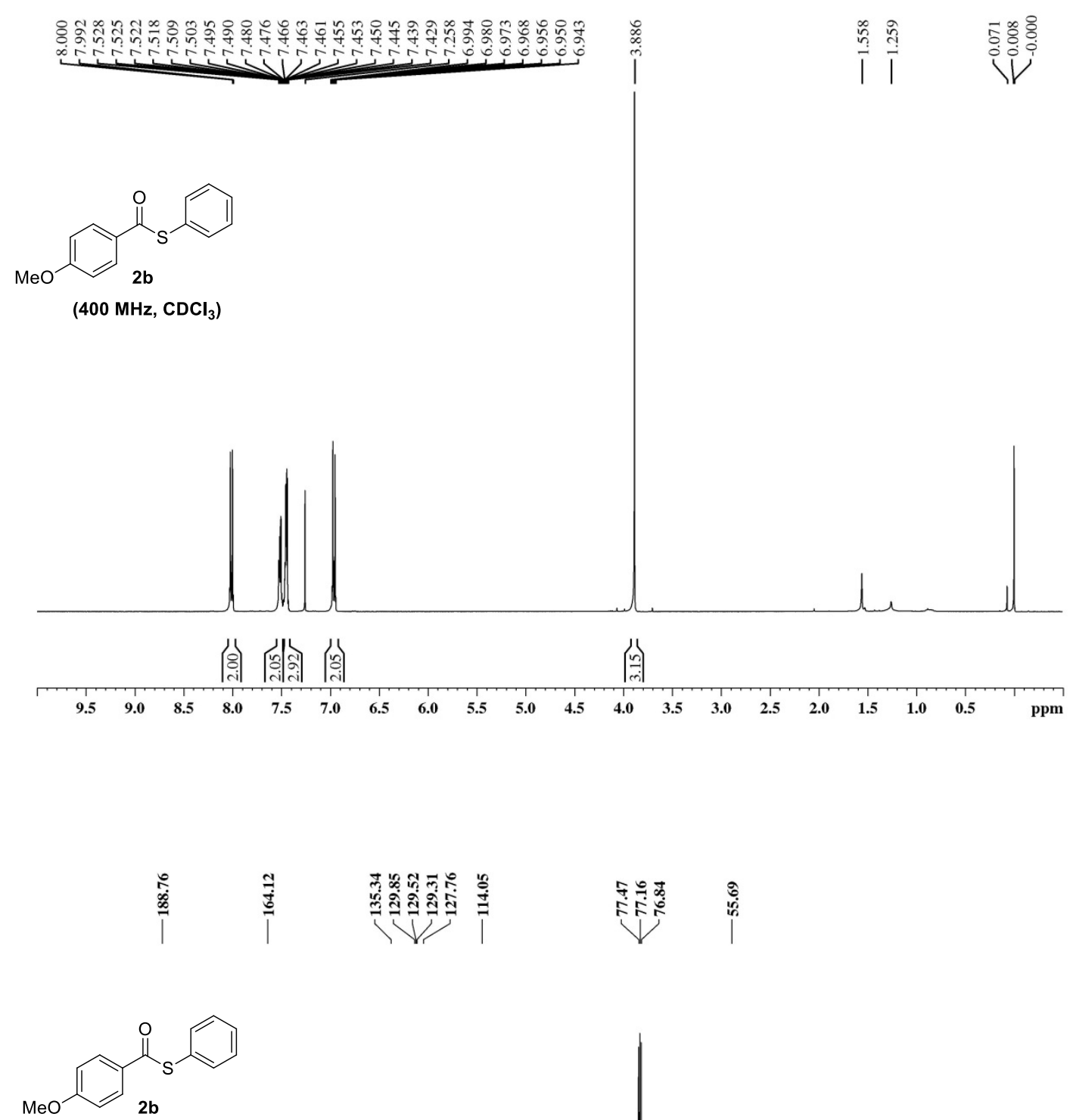

(100 MHz, $\mathrm{CDCl}_{3}$ )
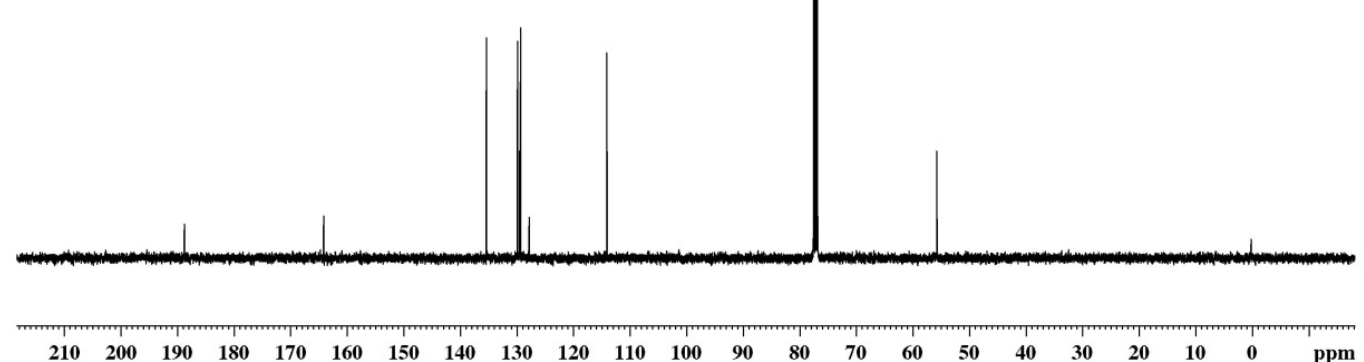
(n)<smiles>O=C(Sc1ccccc1)c1ccc(F)cc1</smiles>

$\left(400 \mathrm{MHz}, \mathrm{CDCl}_{3}\right)$
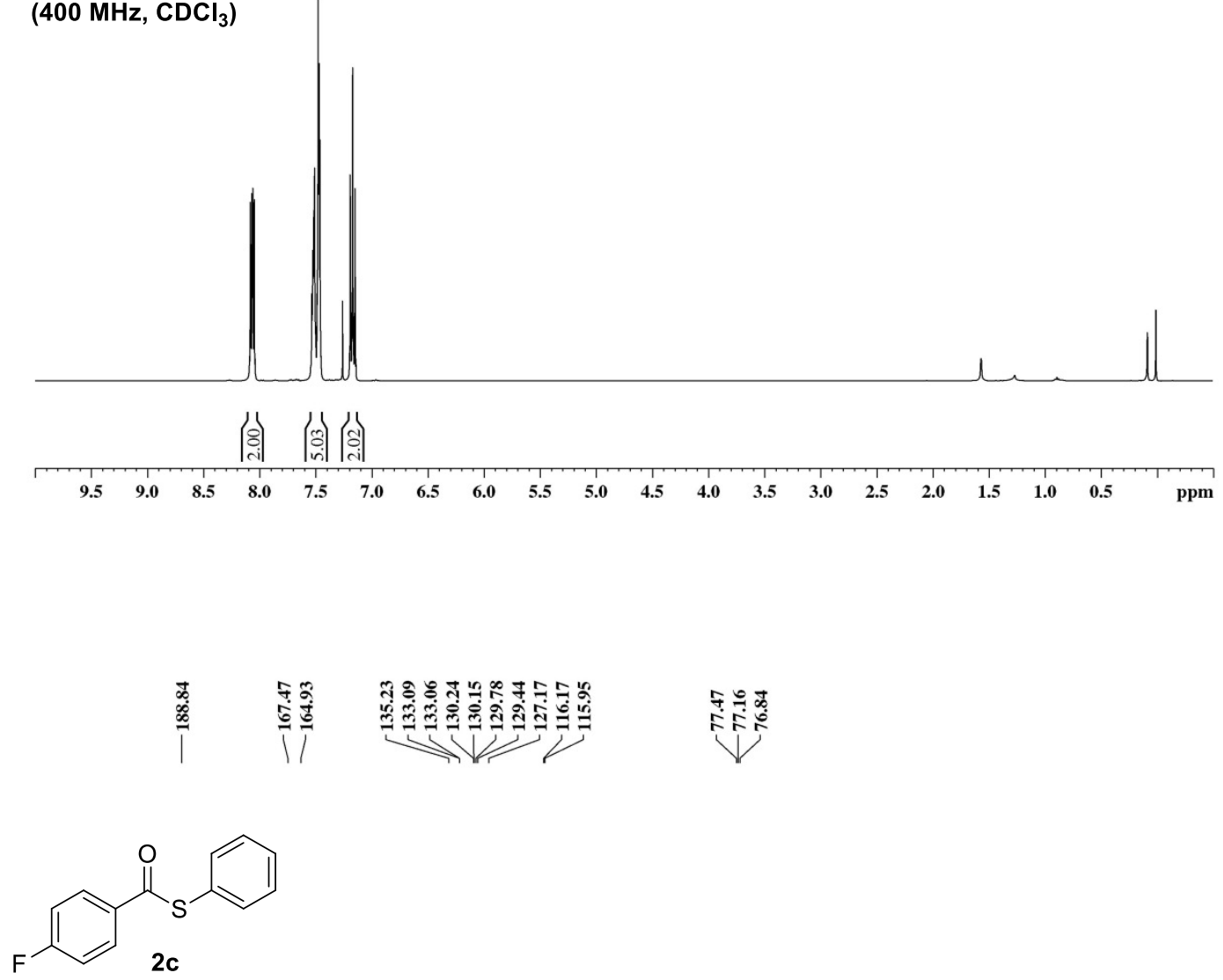

$\left(100 \mathrm{MHz}, \mathrm{CDCl}_{3}\right)$

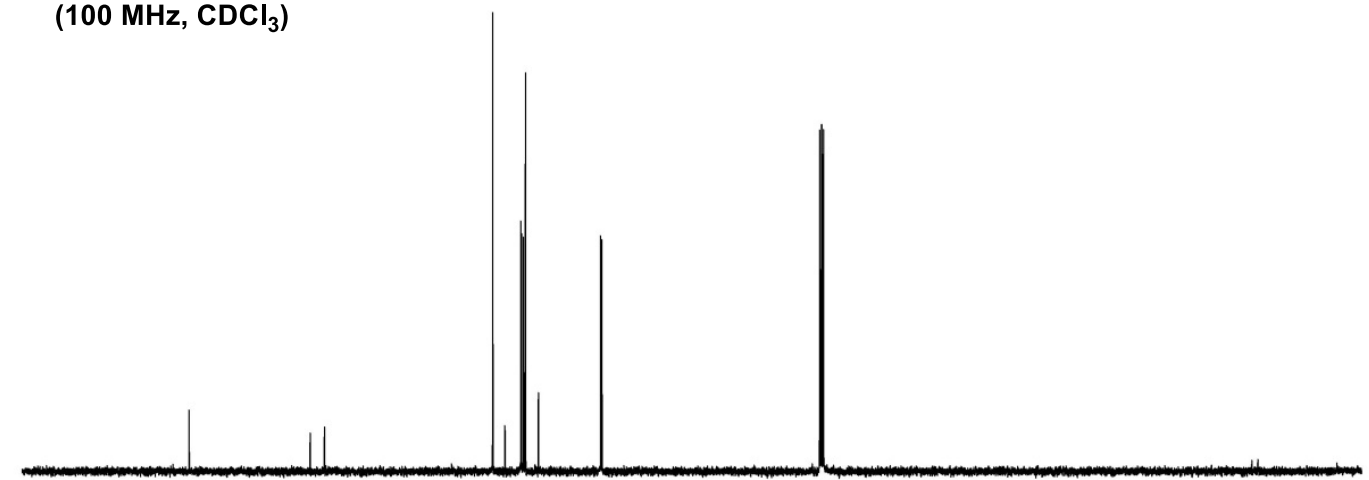

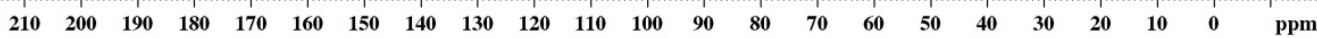




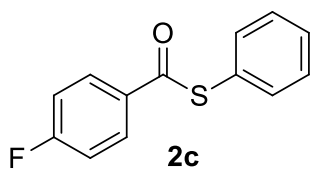

$\frac{a}{i}$

(376 $\mathrm{MHz}, \mathrm{CDCl}_{3}$ )

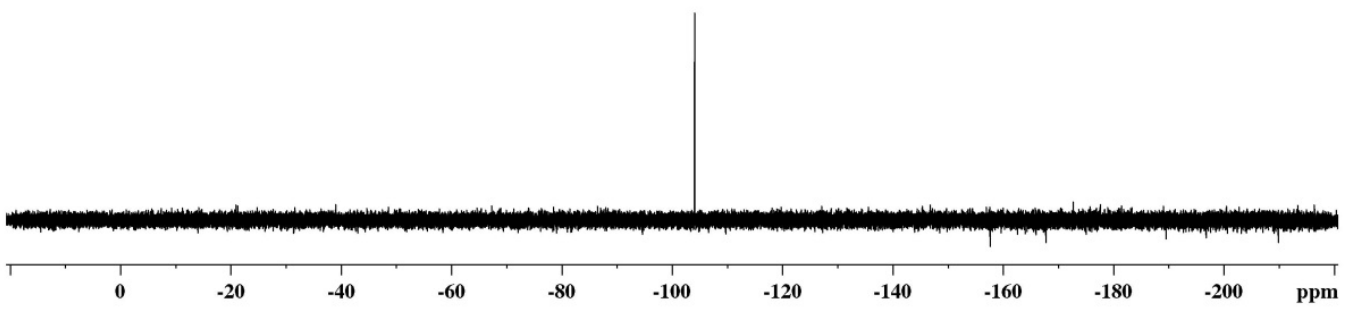


<smiles>O=C(Sc1ccccc1)c1ccc(Cl)cc1</smiles>

$\left(400 \mathrm{MHz}, \mathrm{CDCl}_{3}\right.$ )
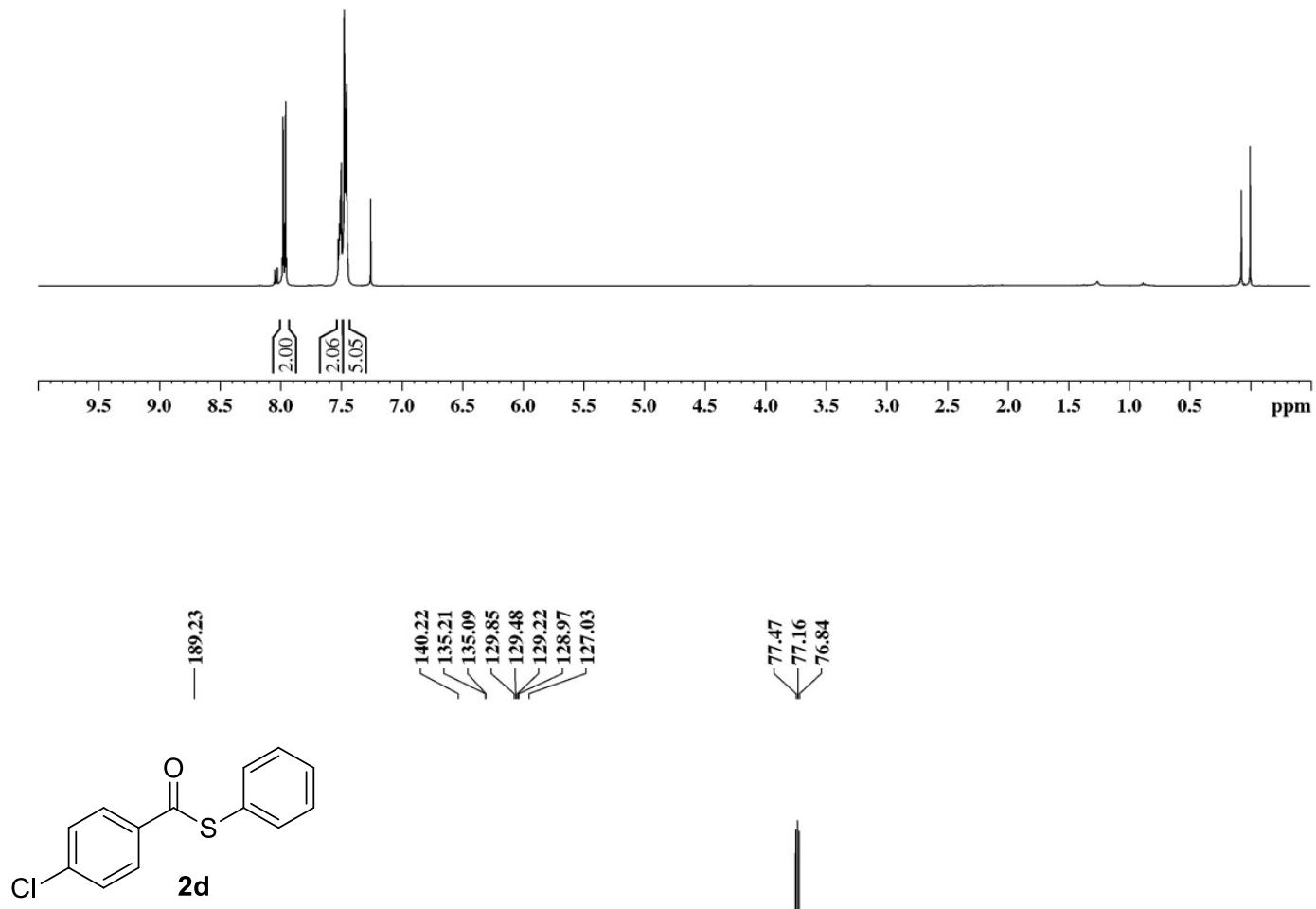

$\left(100 \mathrm{MHz}, \mathrm{CDCl}_{3}\right.$ )

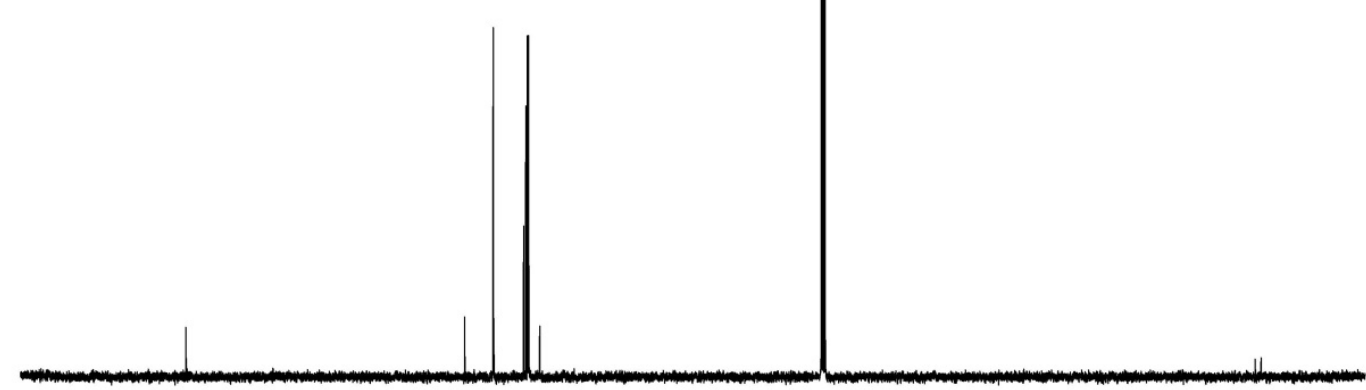




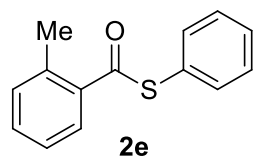

$\left(400 \mathrm{MHz}, \mathrm{CDCl}_{3}\right)$
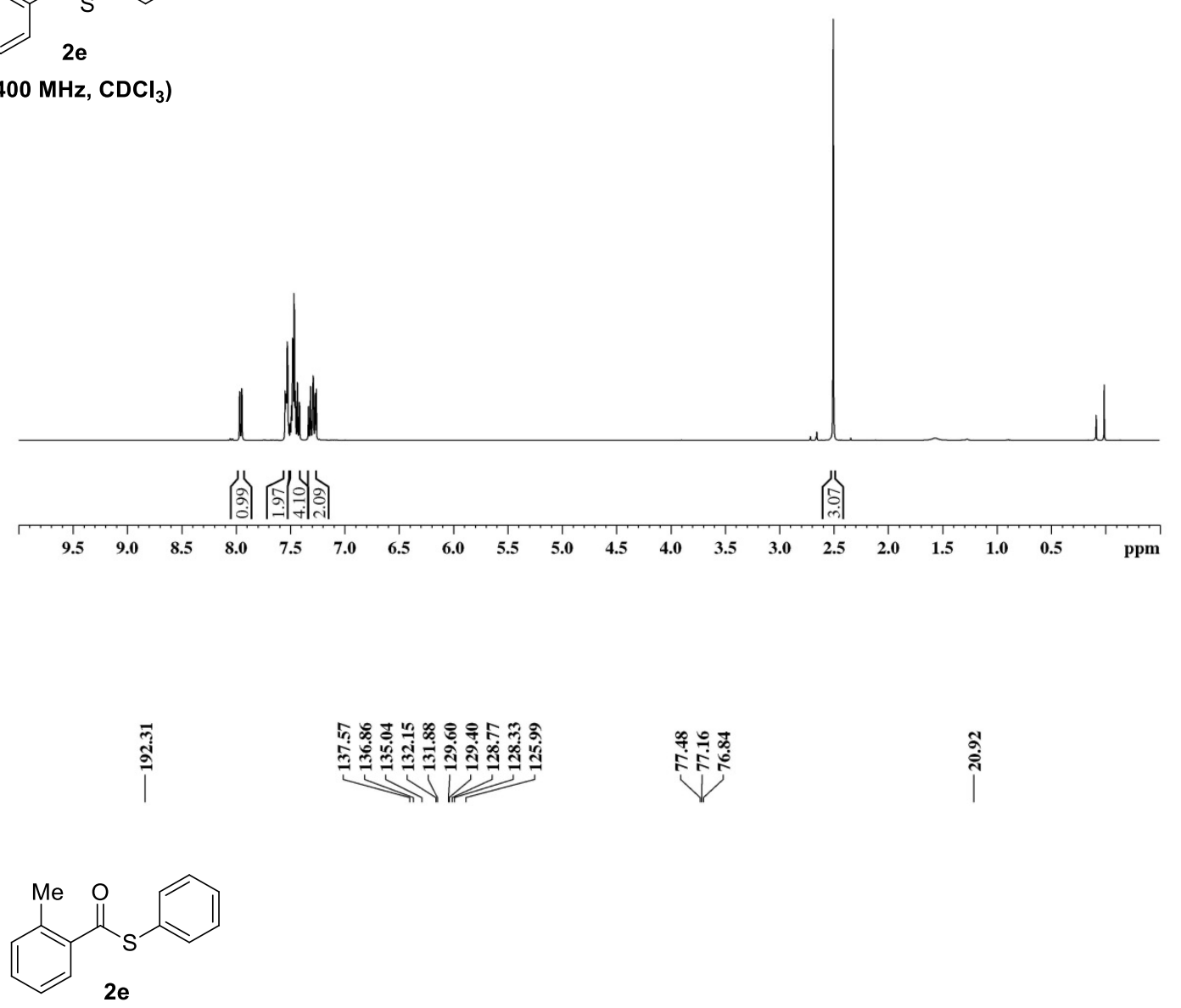

$\left(100 \mathrm{MHz}, \mathrm{CDCl}_{3}\right)$

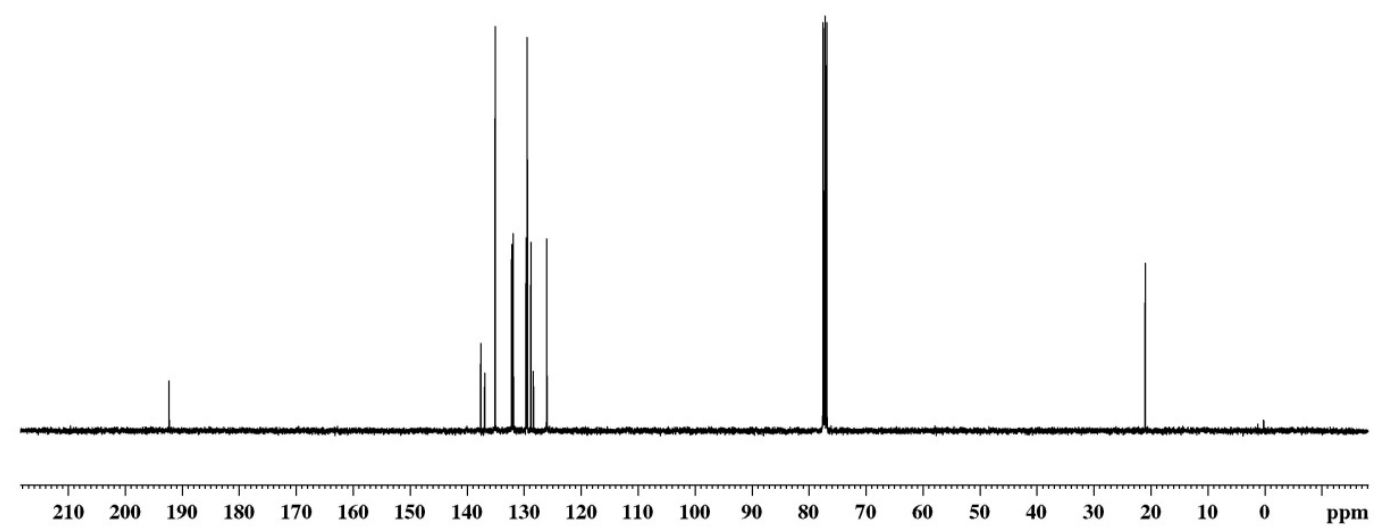




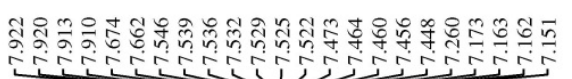

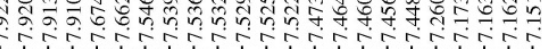

$\longrightarrow$<smiles>O=C(Sc1ccccc1)c1cccs1</smiles>

$\left(400 \mathrm{MHz}, \mathrm{CDCl}_{3}\right.$ )
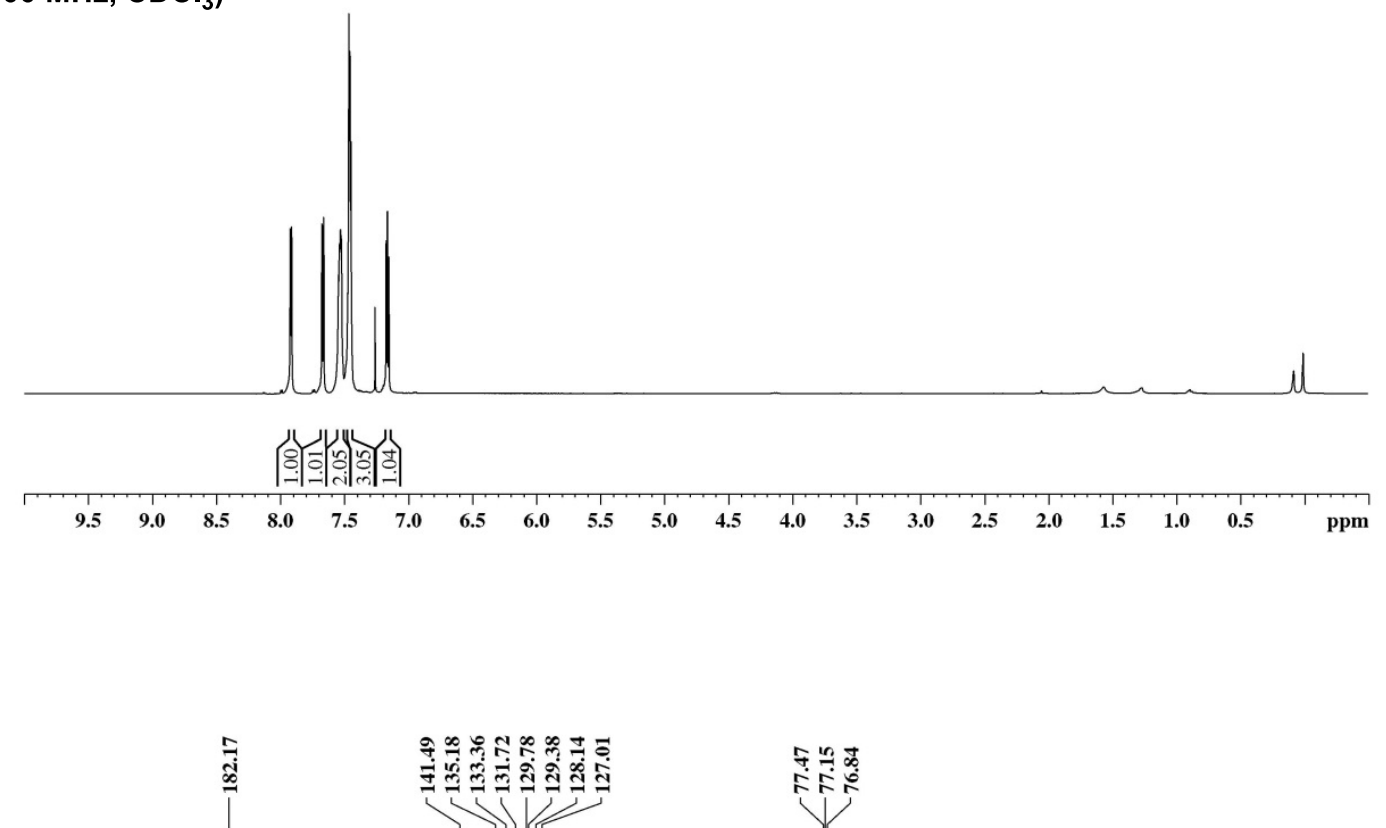

V<smiles>O=C(Sc1ccccc1)c1cccs1</smiles>

$\left(100 \mathrm{MHz}, \mathrm{CDCl}_{3}\right)$

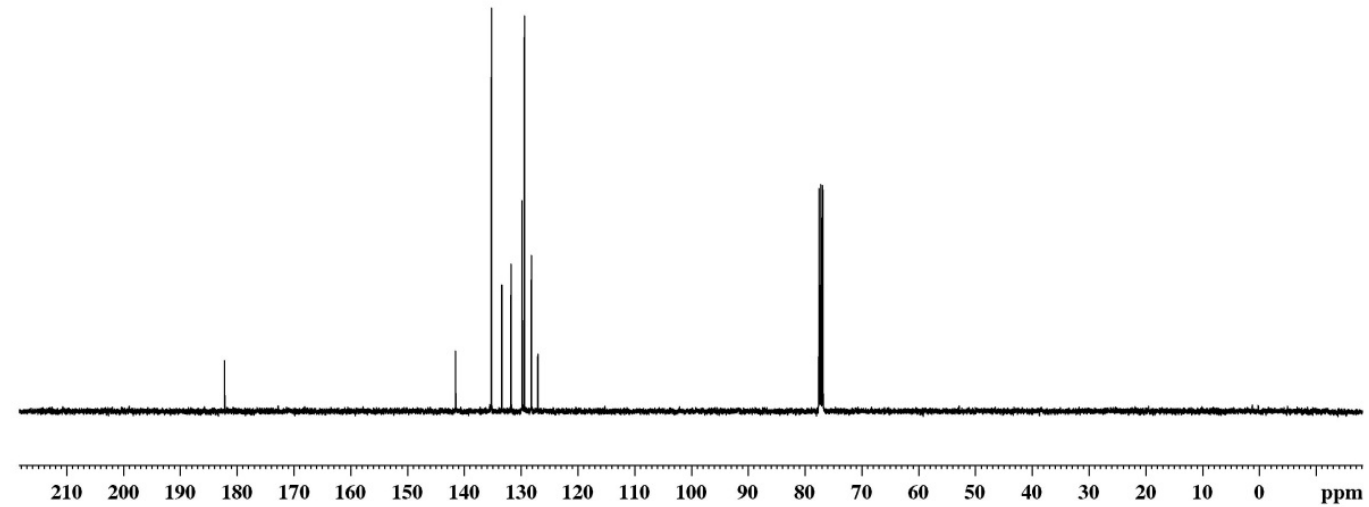




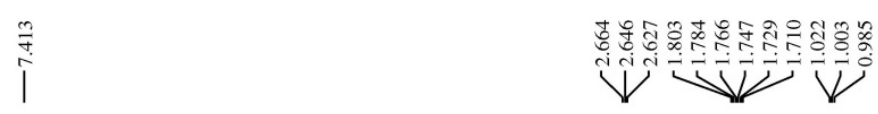<smiles>CCCC(=O)Sc1ccccc1</smiles>

$2 \mathrm{~g}$

$\left(400 \mathrm{MHz}, \mathrm{CDCl}_{3}\right)$
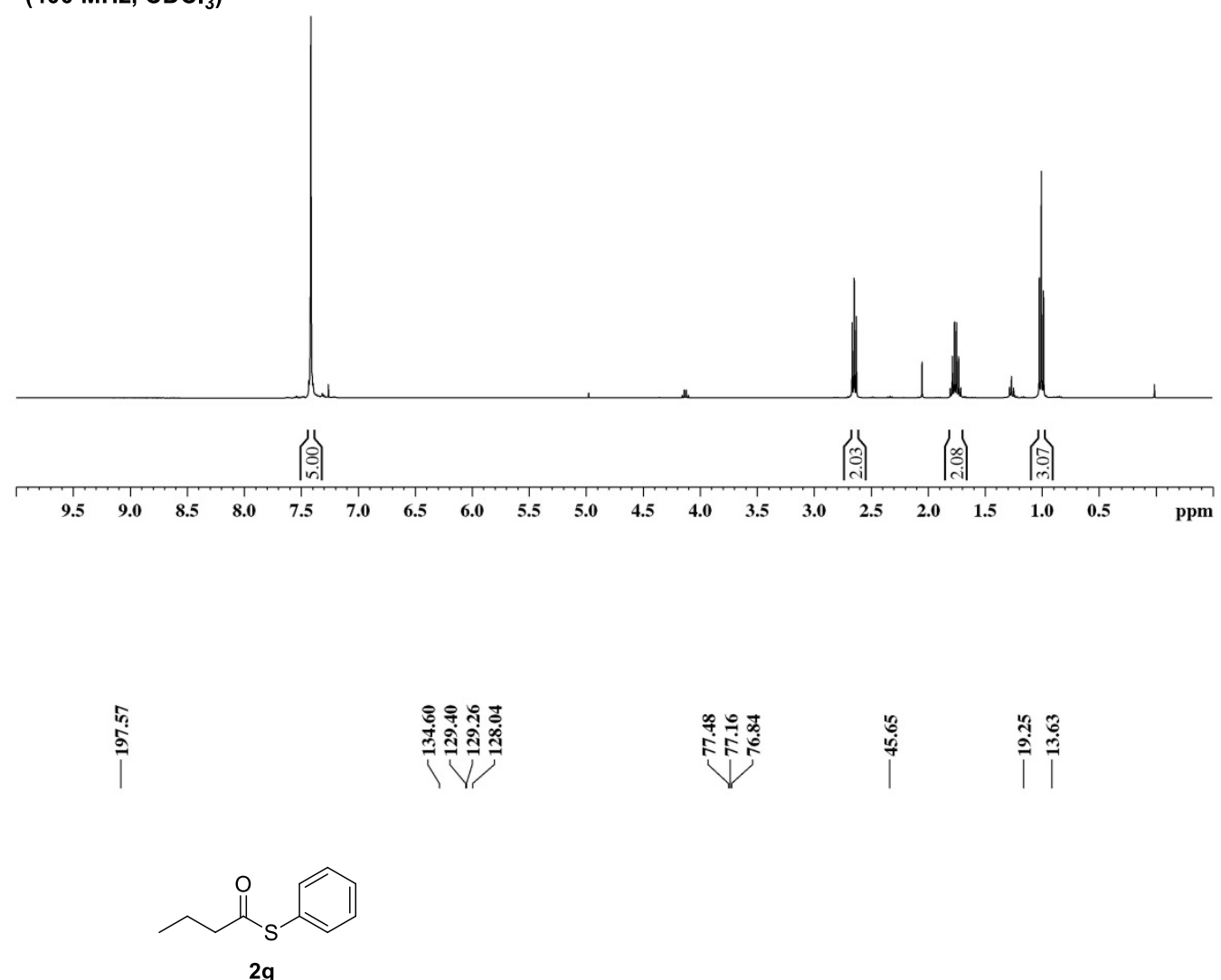

$\left(100 \mathrm{MHz}, \mathrm{CDCl}_{3}\right)$

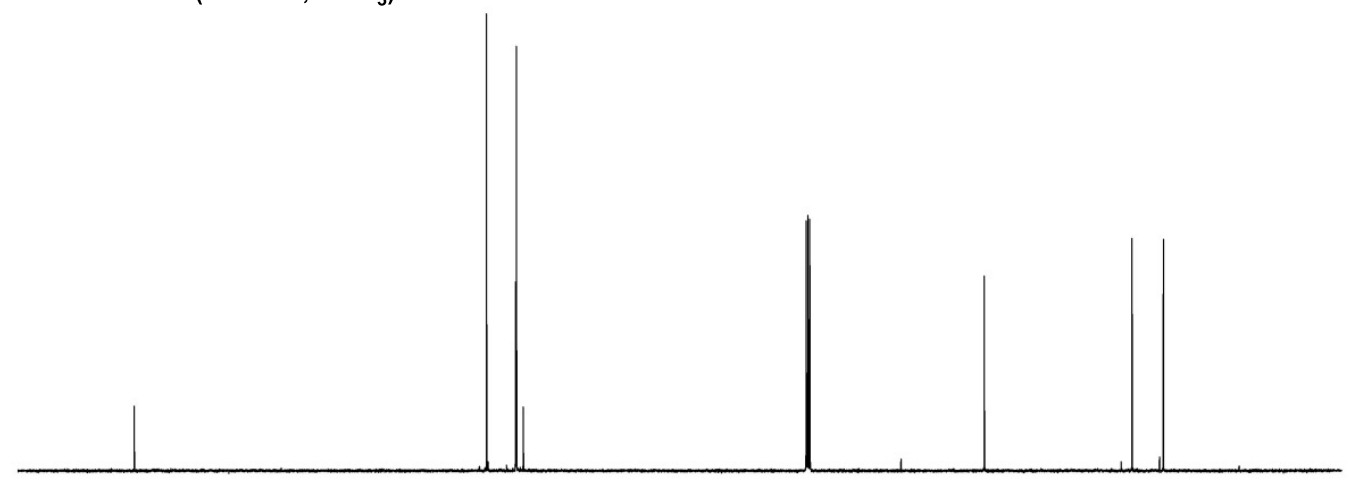

$\begin{array}{llllllllllllllllllllll}210 & 200 & 190 & 180 & 170 & 160 & 150 & 140 & 130 & 120 & 110 & 100 & 90 & 80 & 70 & 60 & 50 & 40 & 30 & 20 & 10 & 0\end{array}$ 

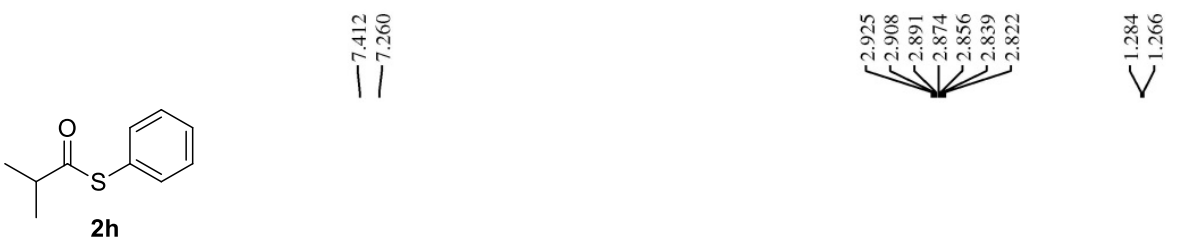

(400 MHz, $\mathrm{CDCl}_{3}$ )
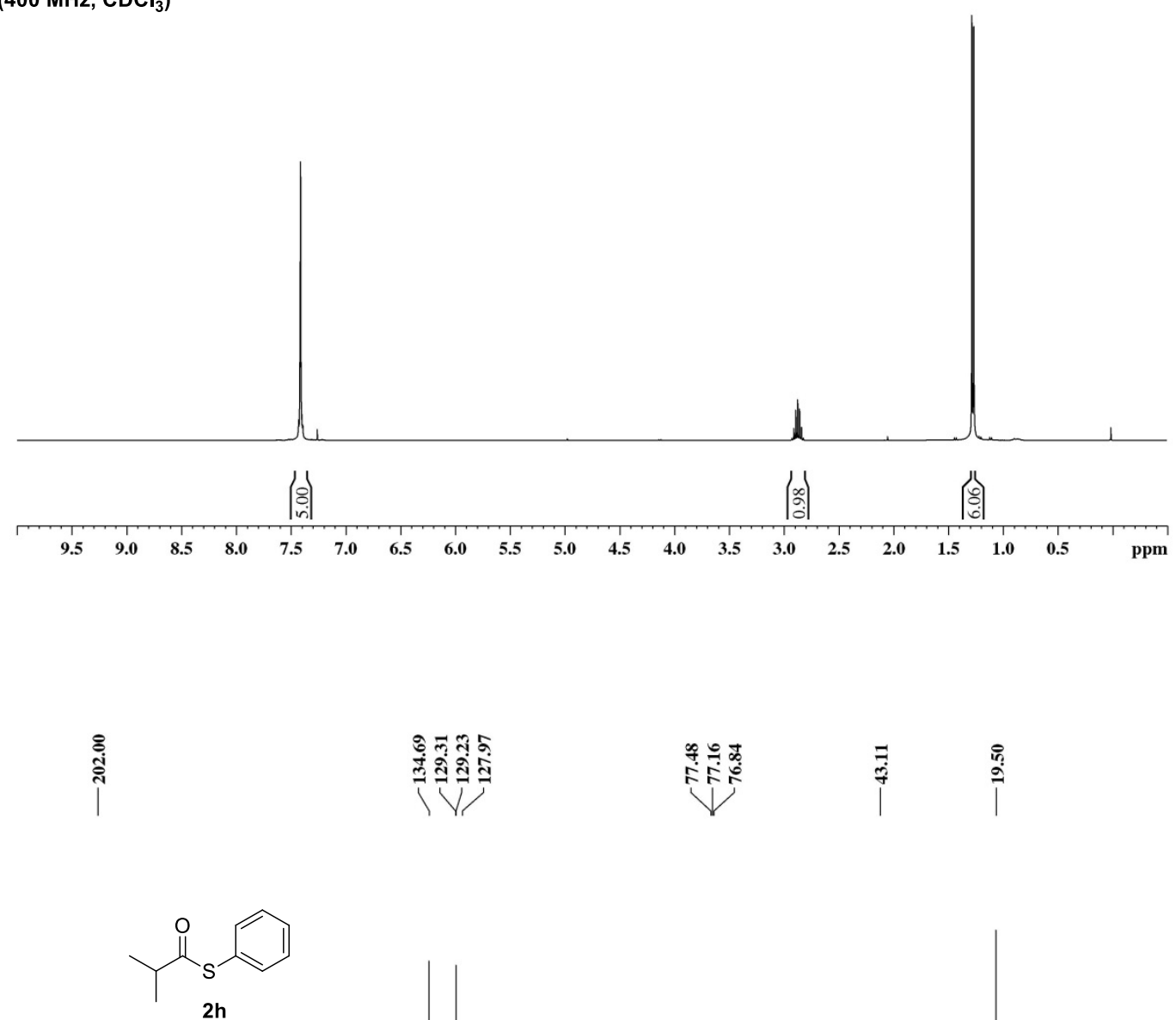

(100 MHz, $\mathrm{CDCl}_{3}$ )

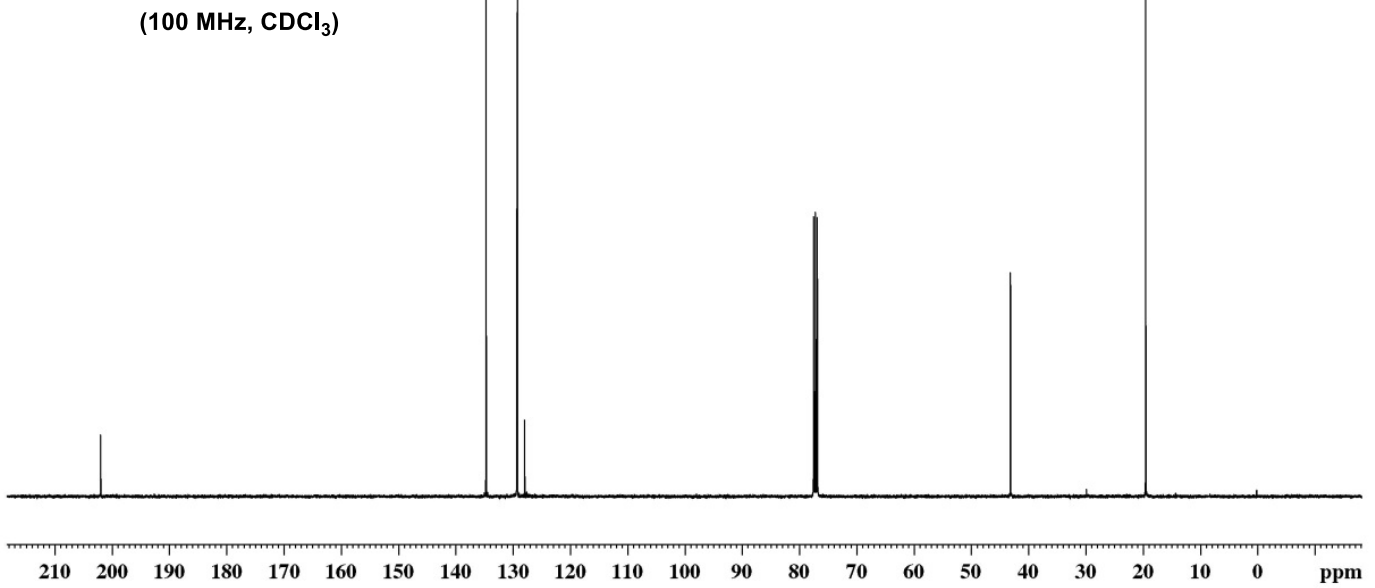




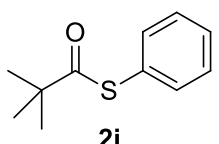

$\left(400 \mathrm{MHz}, \mathrm{CDCl}_{3}\right)$

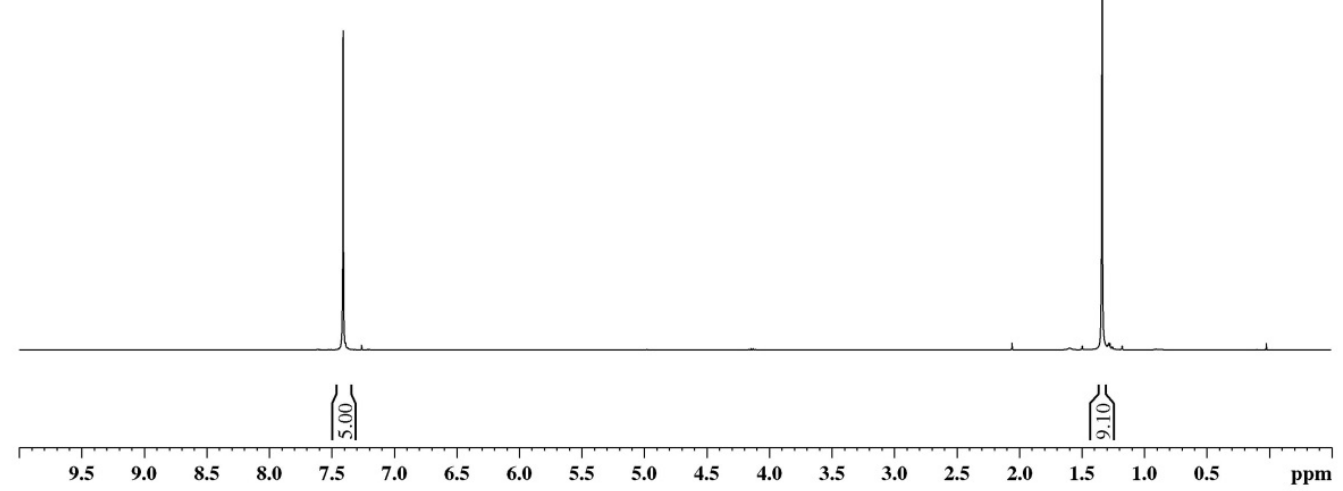

|

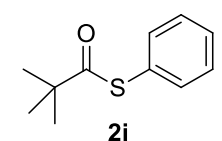

$\left(100 \mathrm{MHz}, \mathrm{CDCl}_{3}\right)$

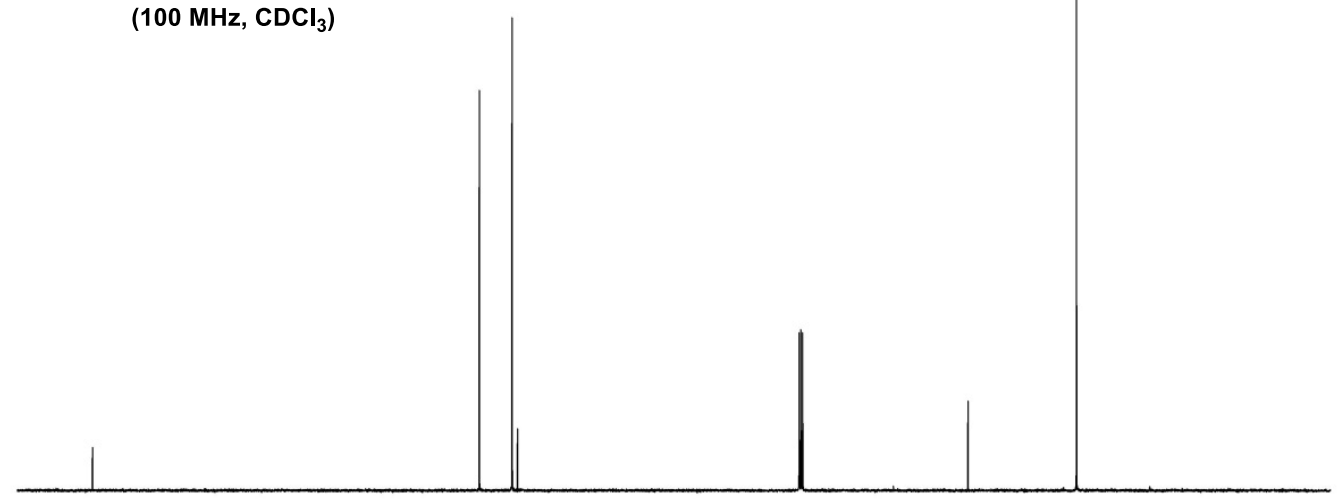

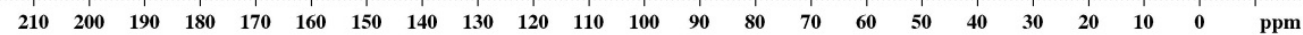



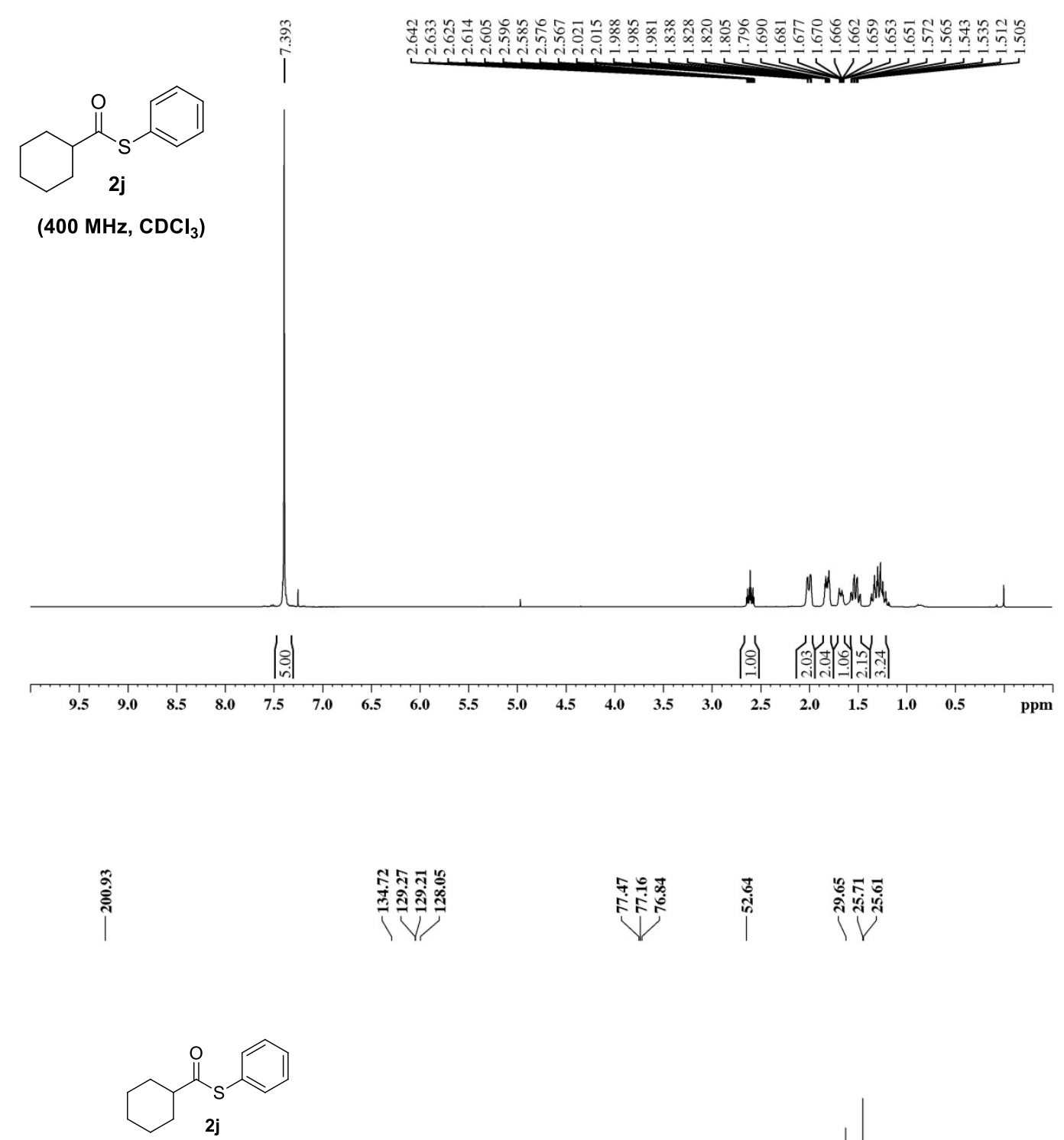

$\left(100 \mathrm{MHz}, \mathrm{CDCl}_{3}\right)$

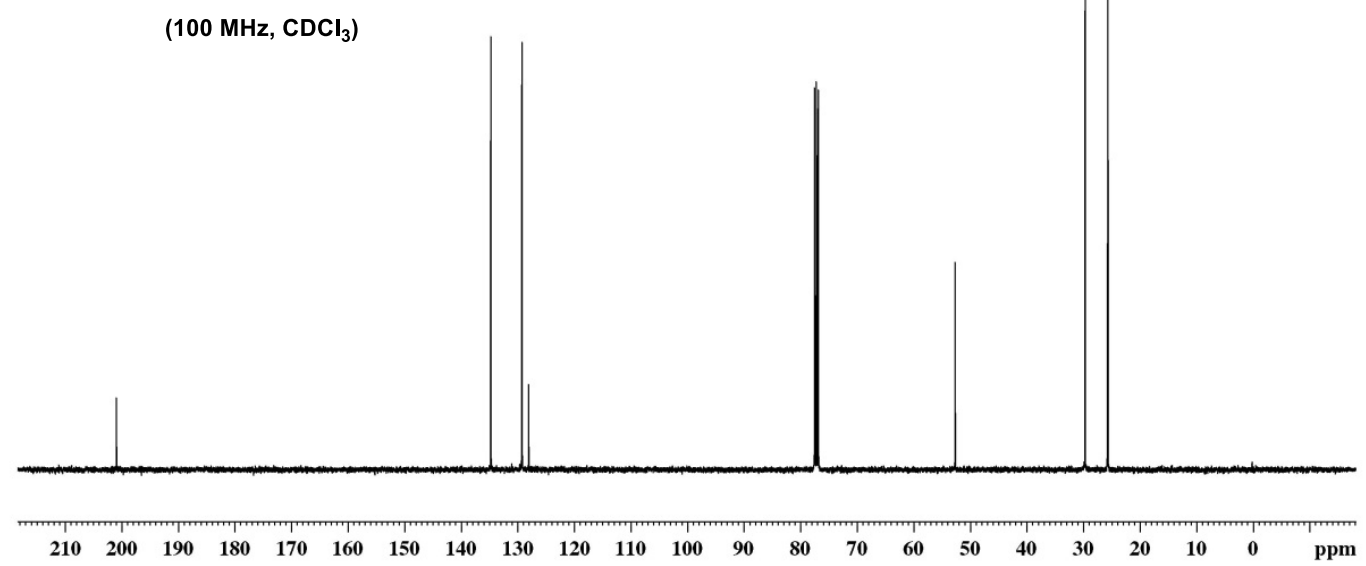



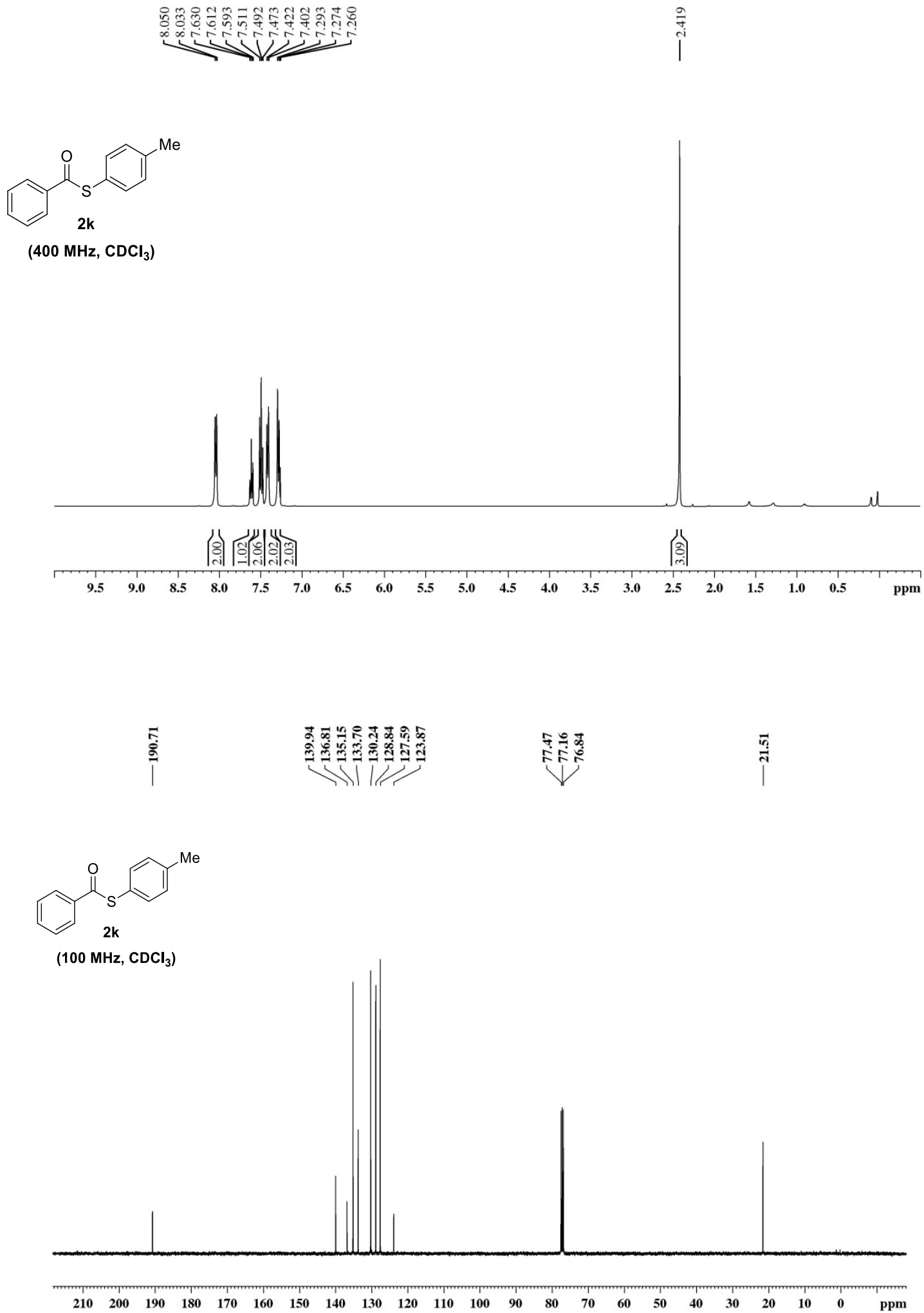

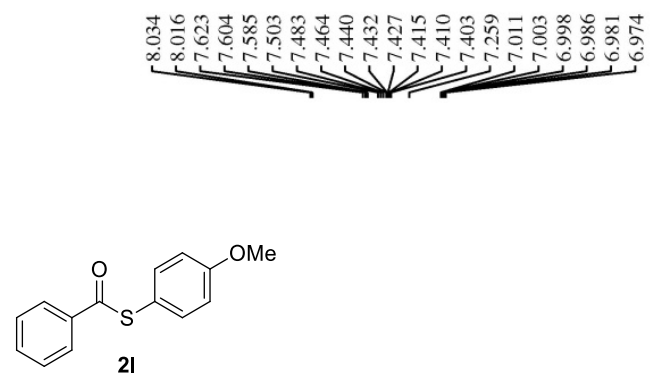

$\left(400 \mathrm{MHz}, \mathrm{CDCl}_{3}\right)$
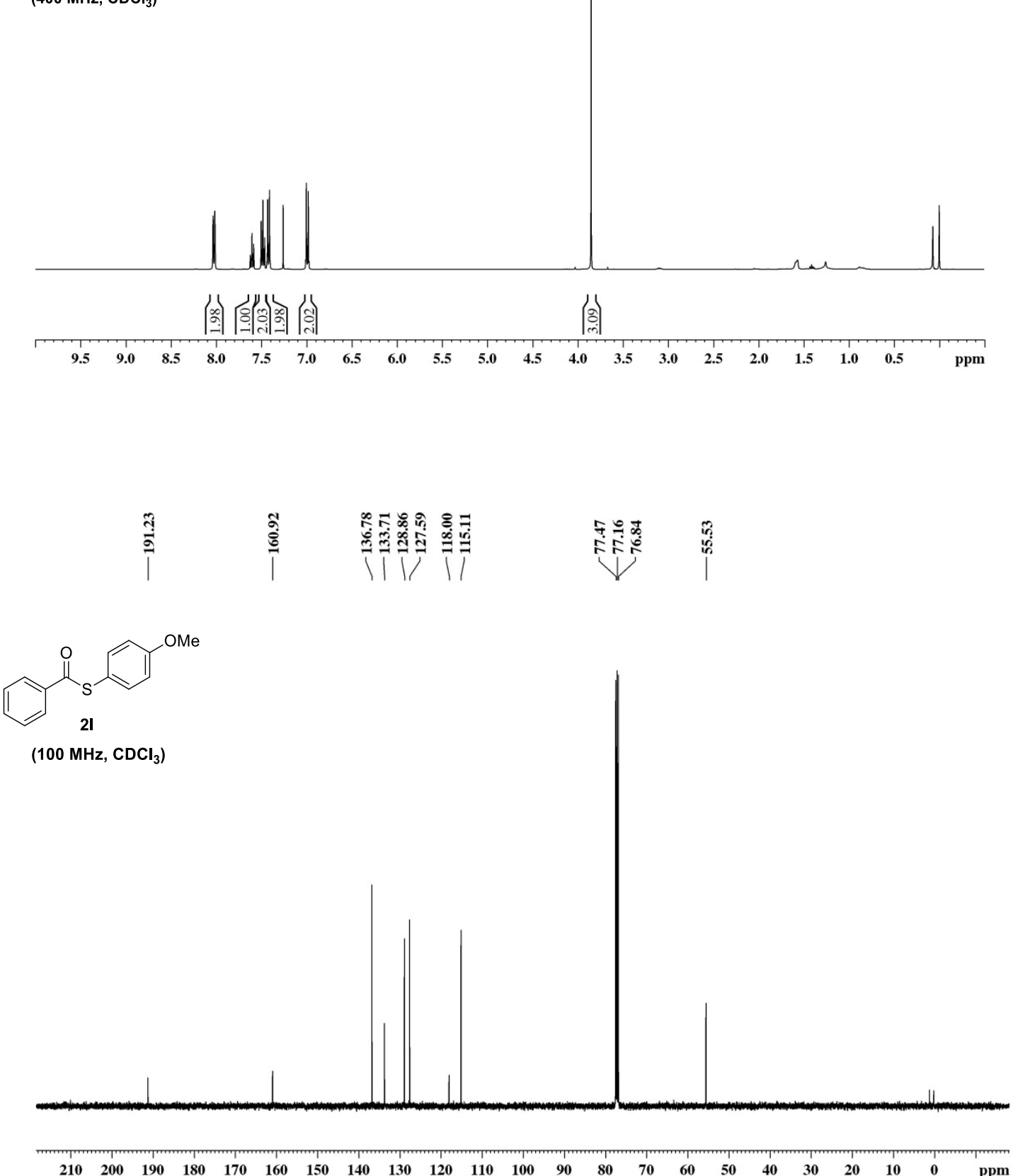


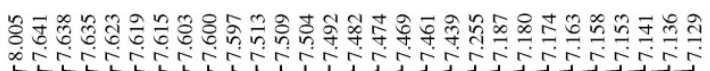

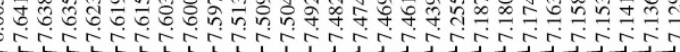

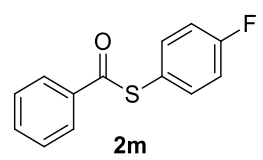

$\left(400 \mathrm{MHz}, \mathrm{CDCl}_{3}\right.$ )
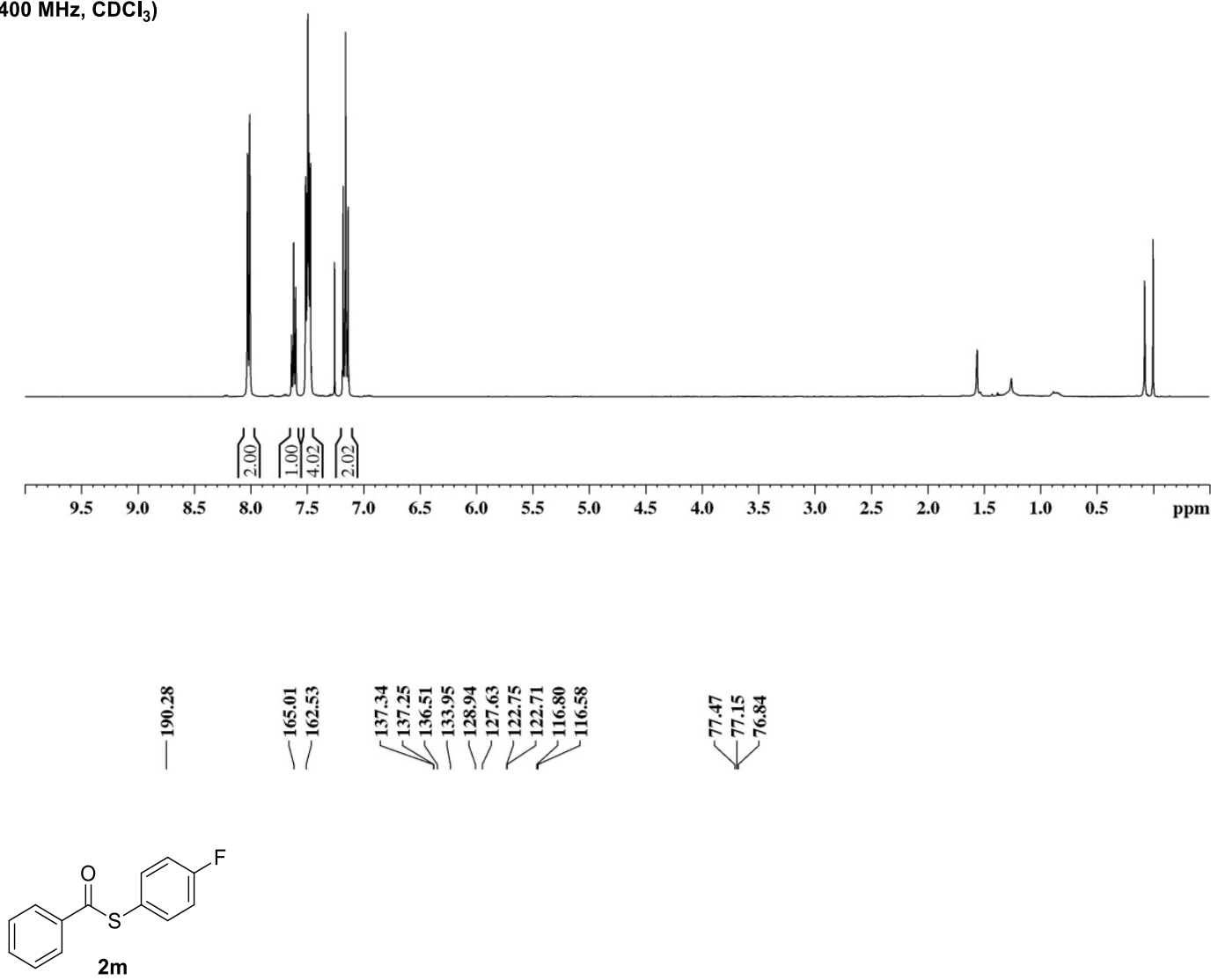

$\left(100 \mathrm{MHz}^{\mathrm{CDCl}_{3}}\right.$ )
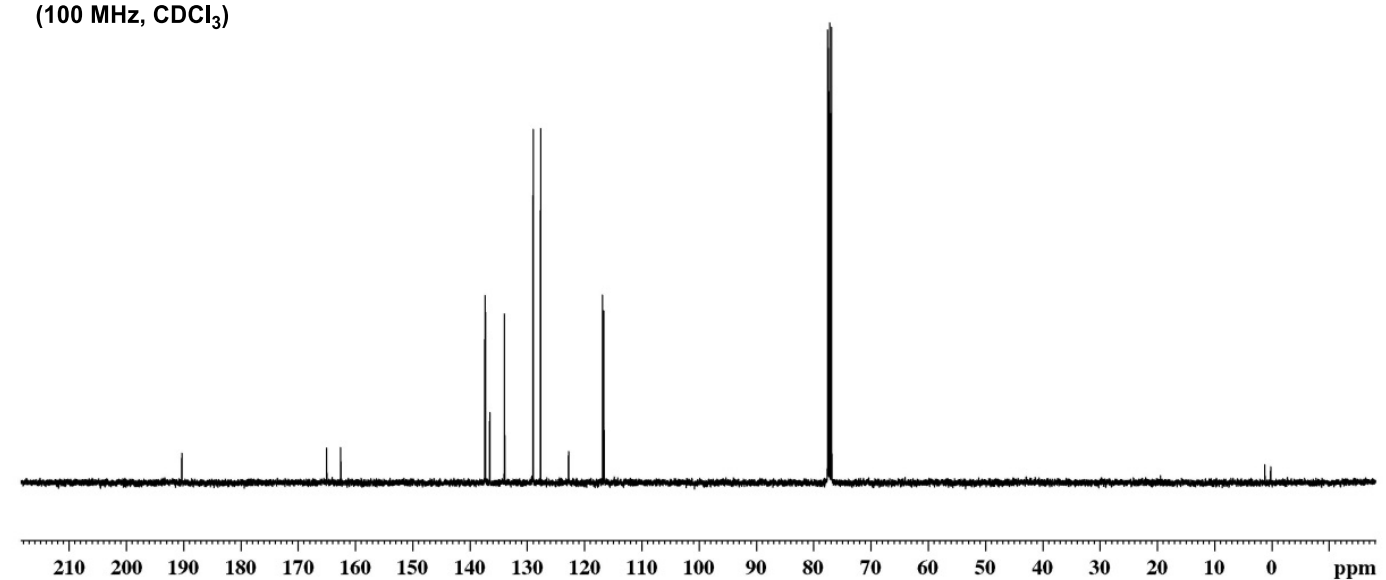


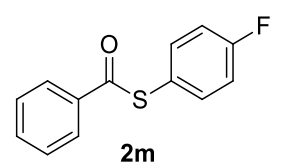

$\left(376 \mathrm{MHz}, \mathrm{CDCl}_{3}\right)$

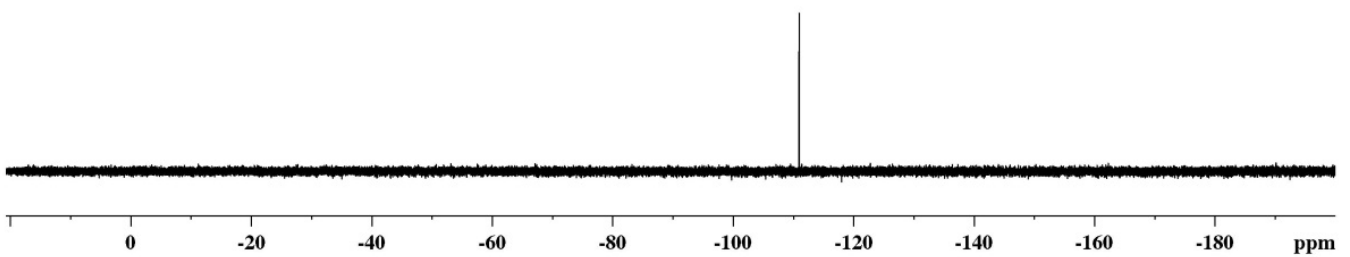




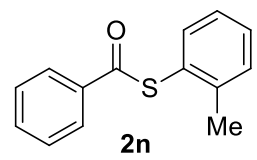

(400 $\mathrm{MHz} \mathrm{CDCl}_{3}$ )

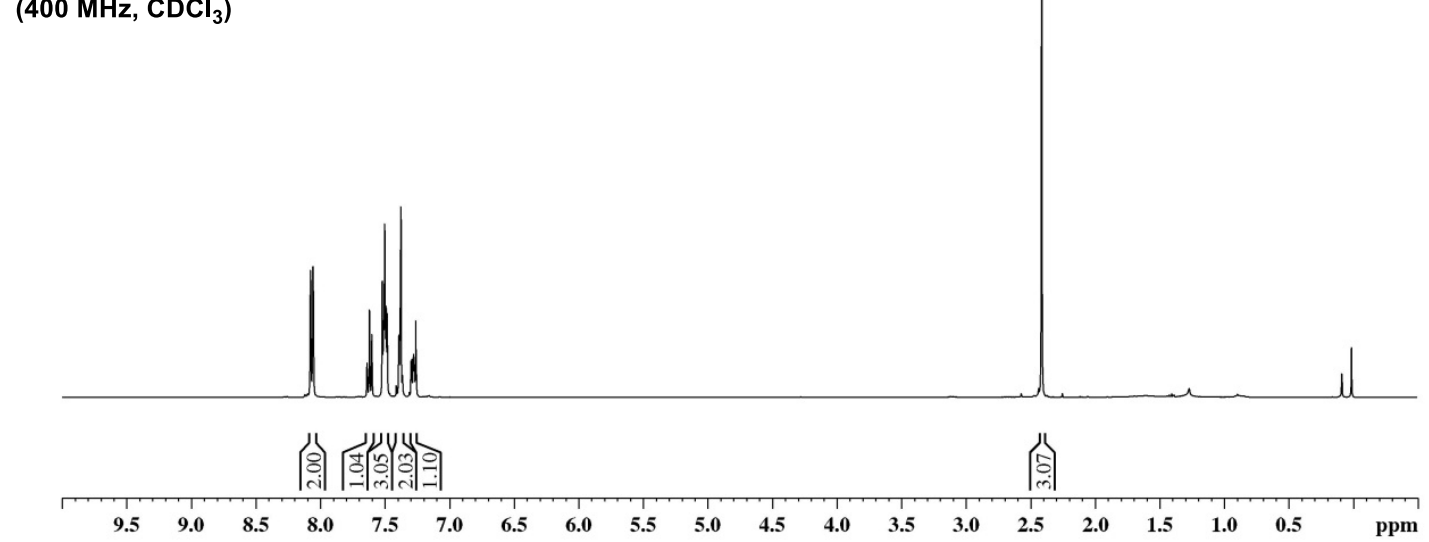

|
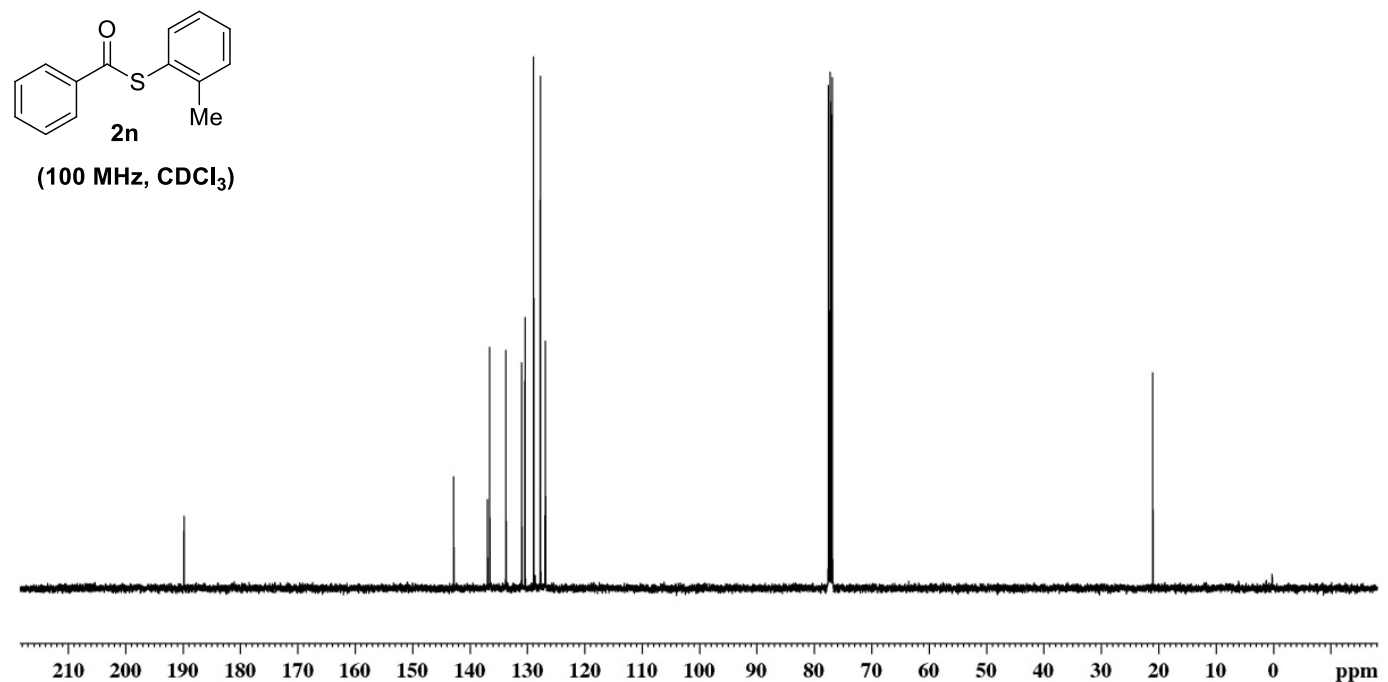

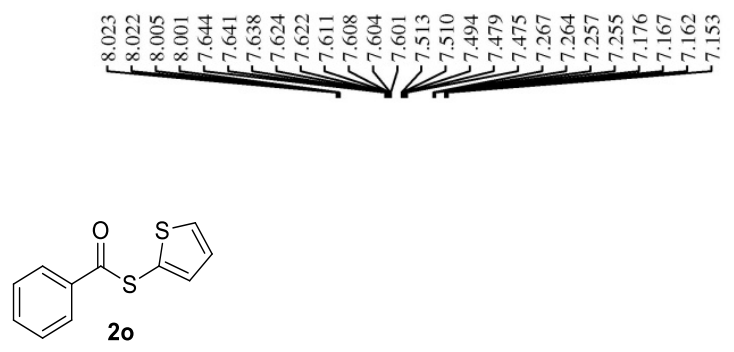

$\left(400 \mathrm{MHz}, \mathrm{CDCl}_{3}\right)$
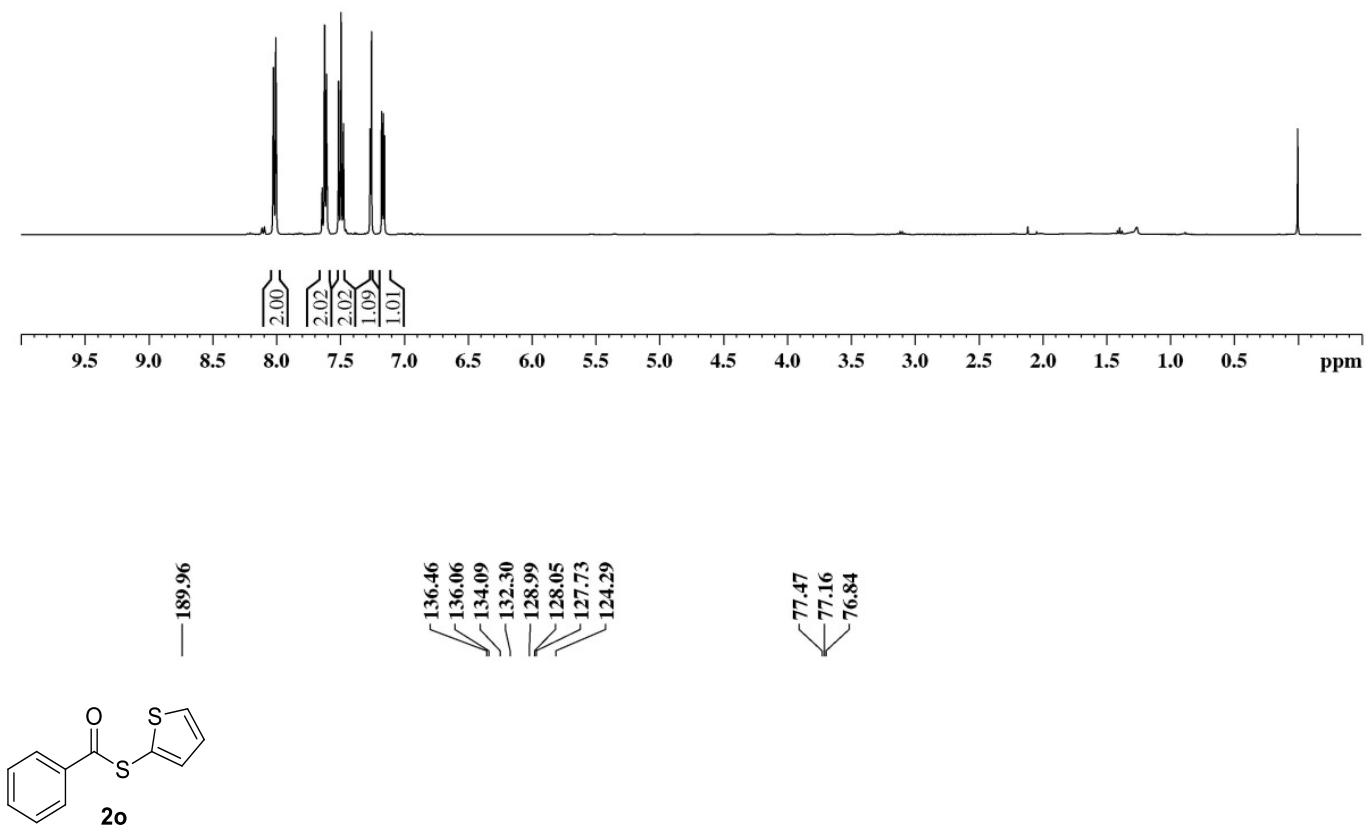

$\left(100 \mathrm{MHz}, \mathrm{CDCl}_{3}\right)$

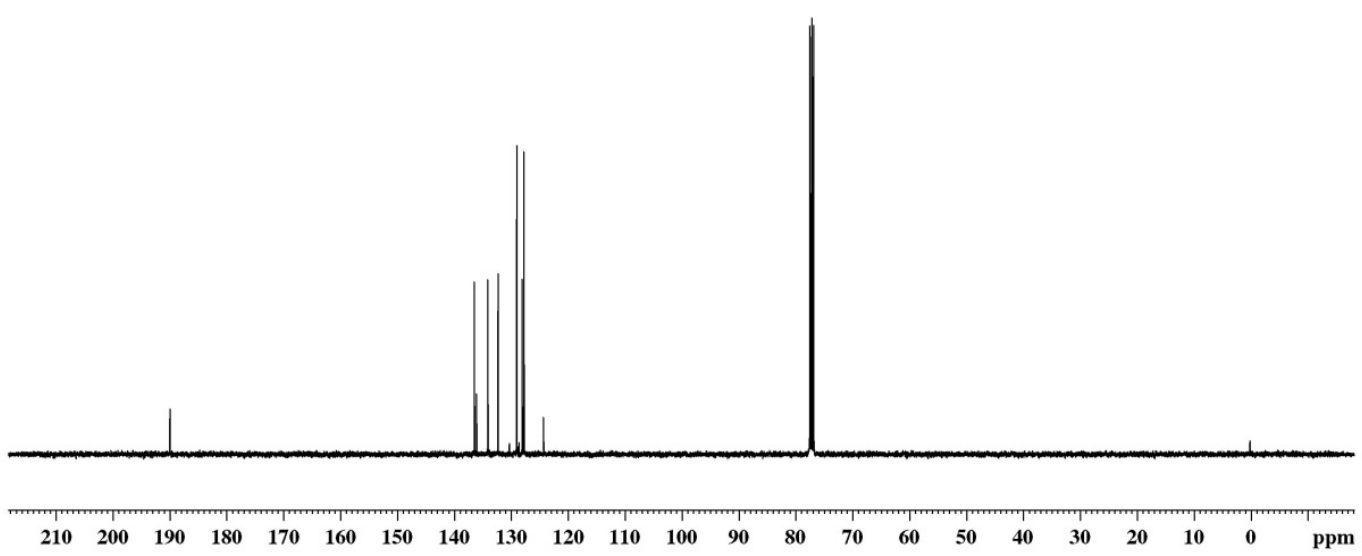



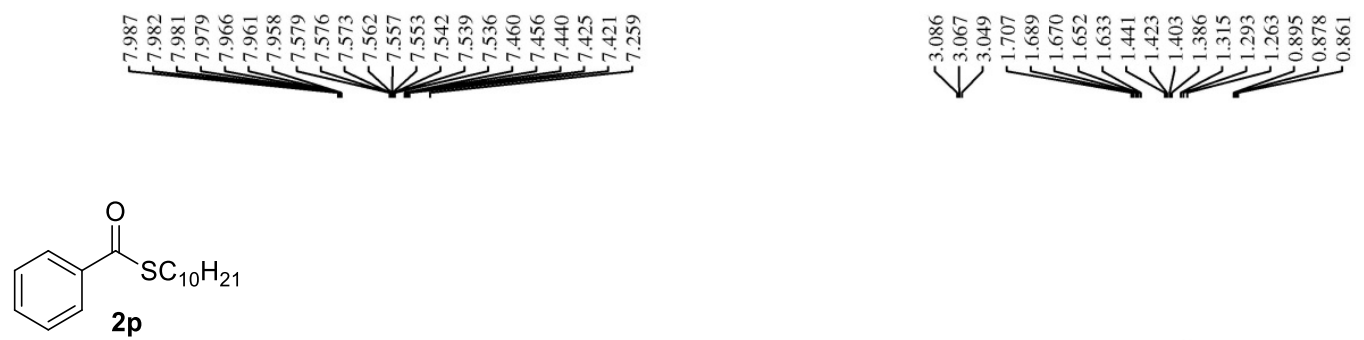

$\left(400 \mathrm{MHz}, \mathrm{CDCl}_{3}\right.$ )
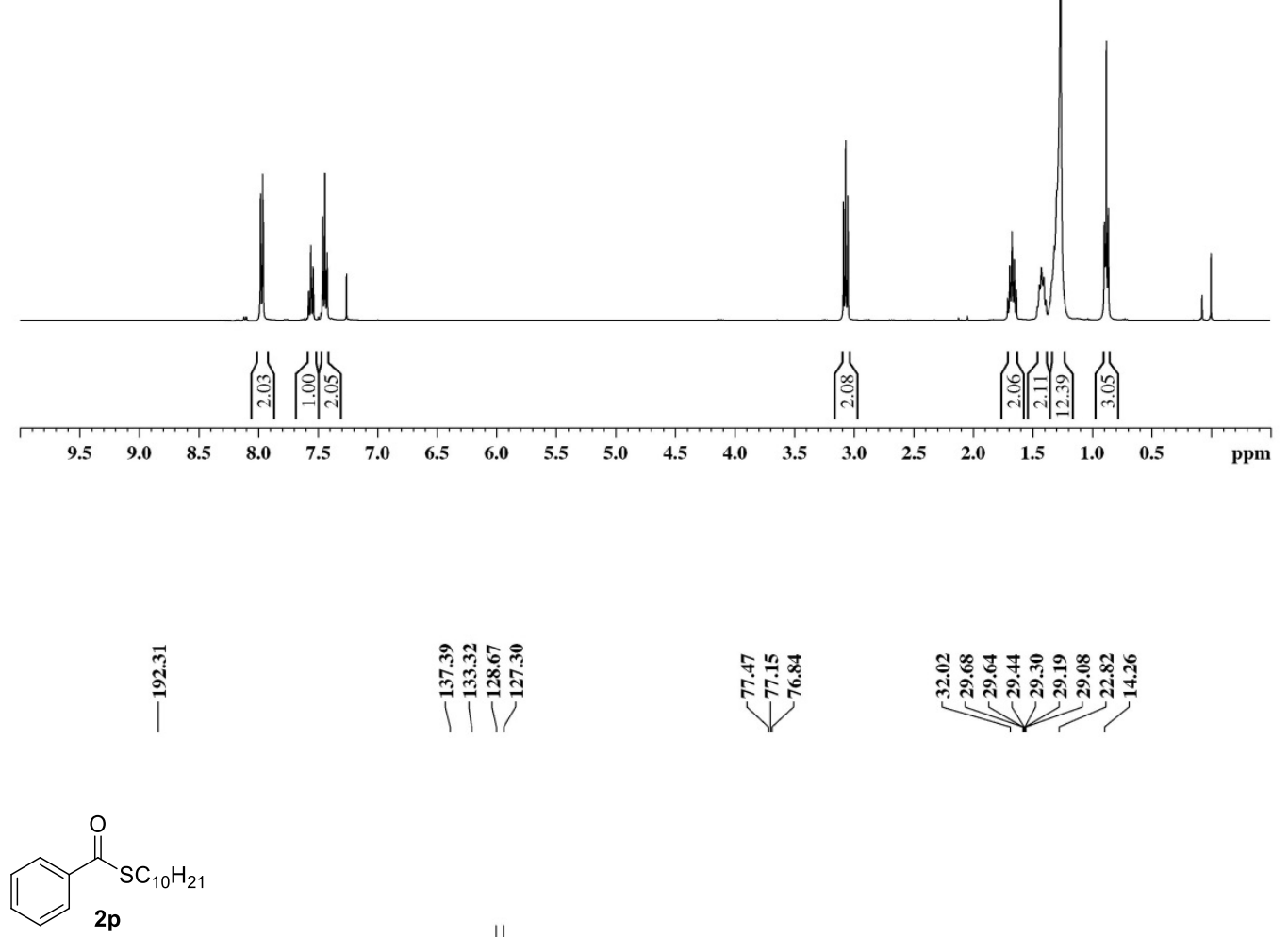

(100 MHz, $\mathrm{CDCl}_{3}$ )

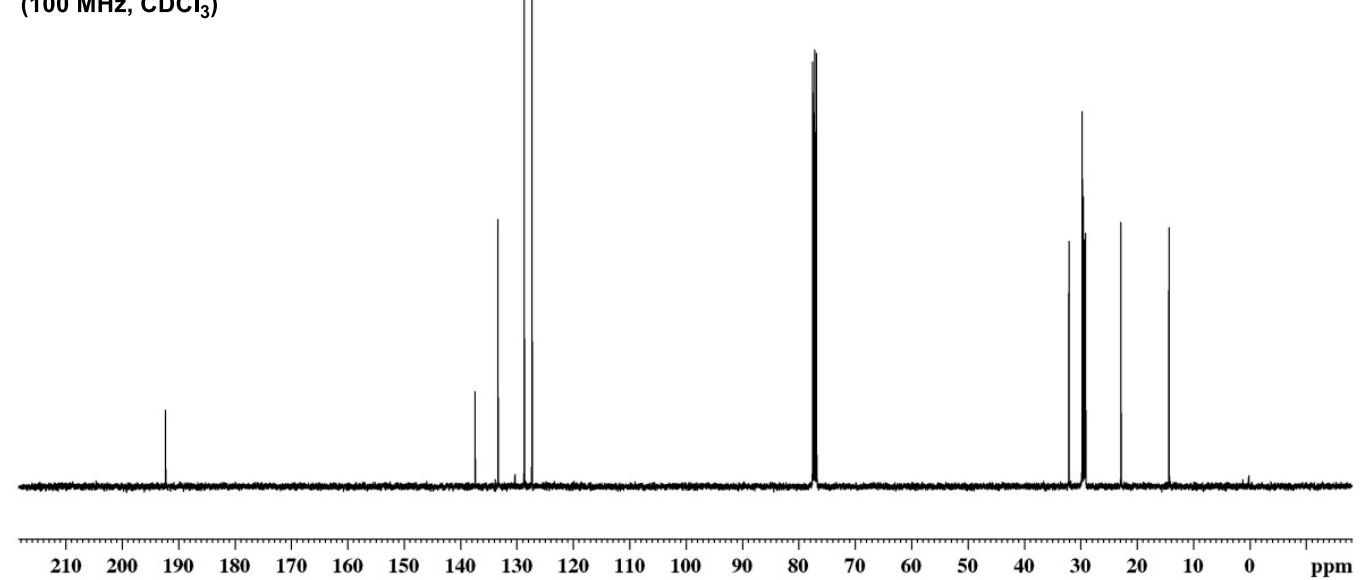




\section{${ }^{1} \mathrm{H},{ }^{13} \mathrm{C}$ and ${ }^{19} \mathrm{~F}$ NMR Spectra of Thioethers}

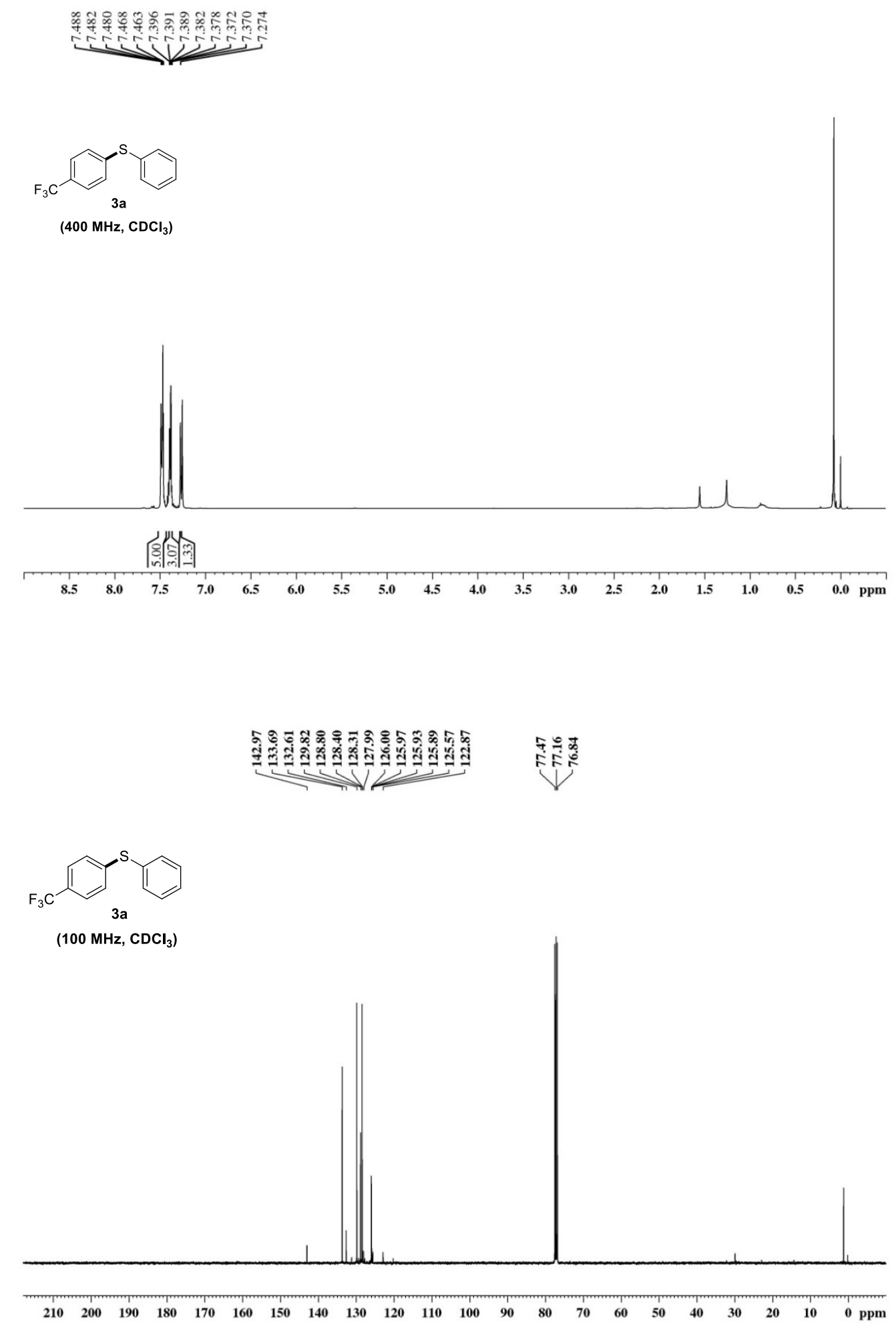




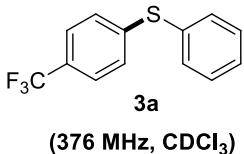

$\left(376 \mathrm{MHz}, \mathrm{CDCl}_{3}\right)$

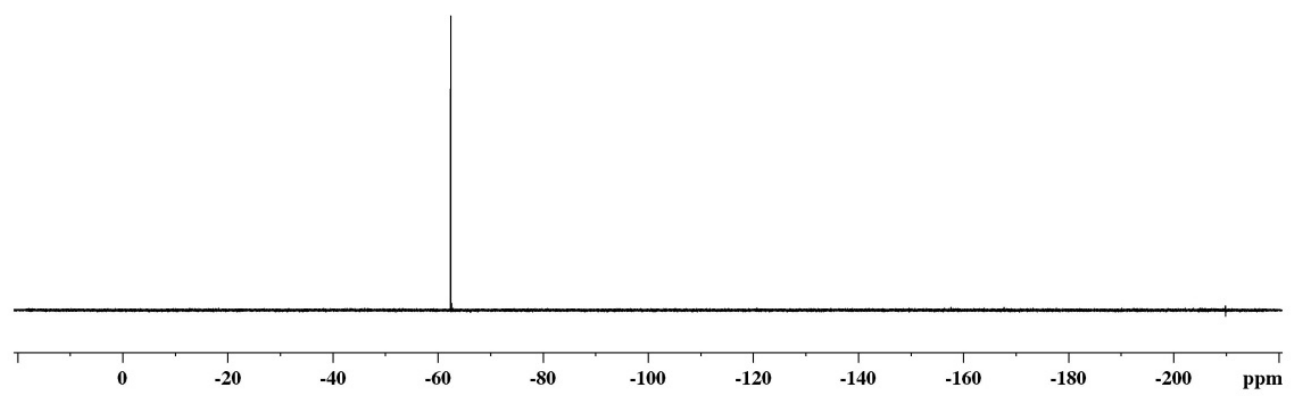




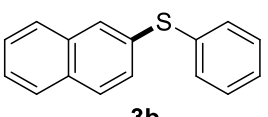

3b

(400 $\mathrm{MHz} \mathrm{CDCl}_{3}$ )
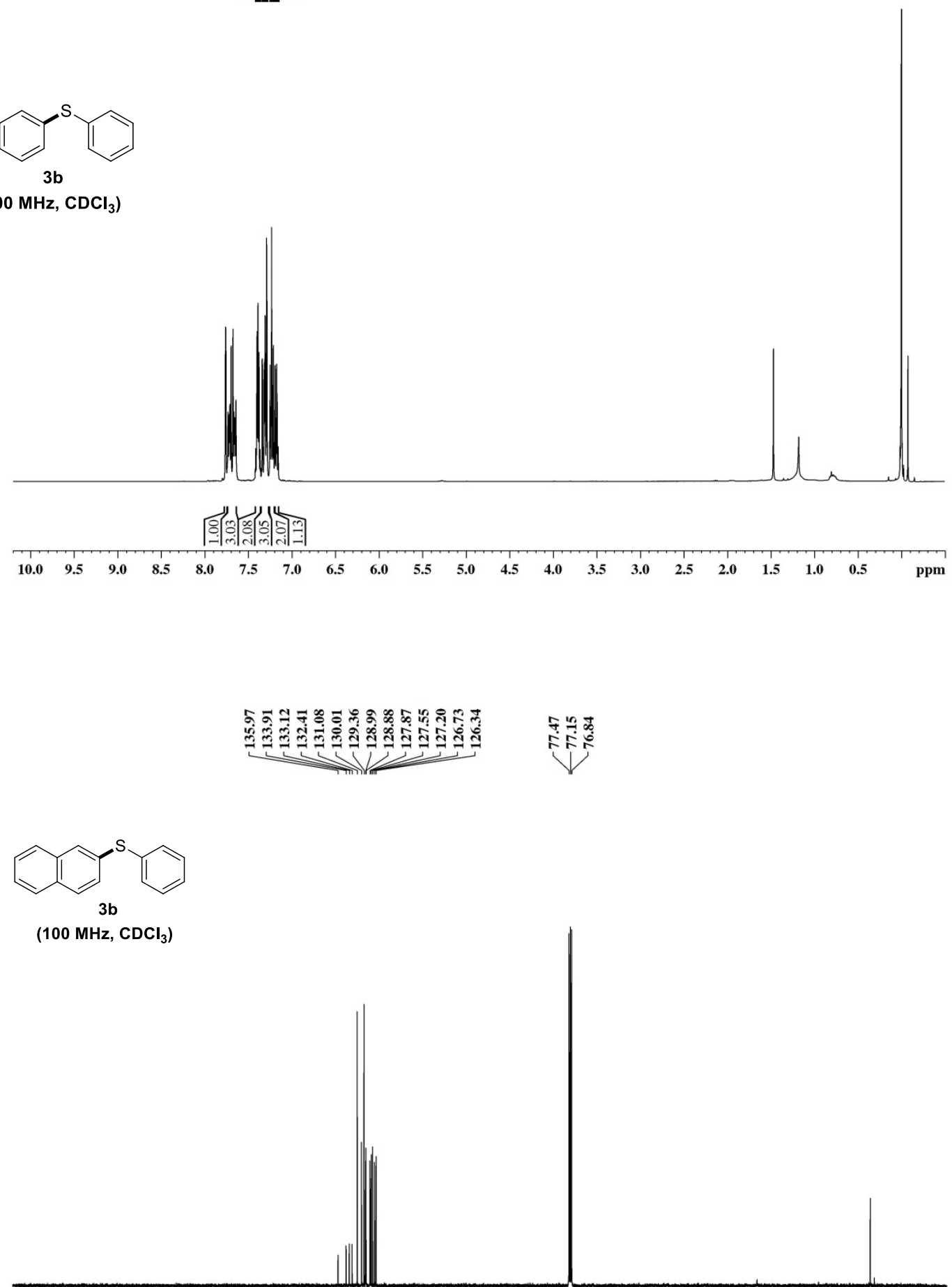

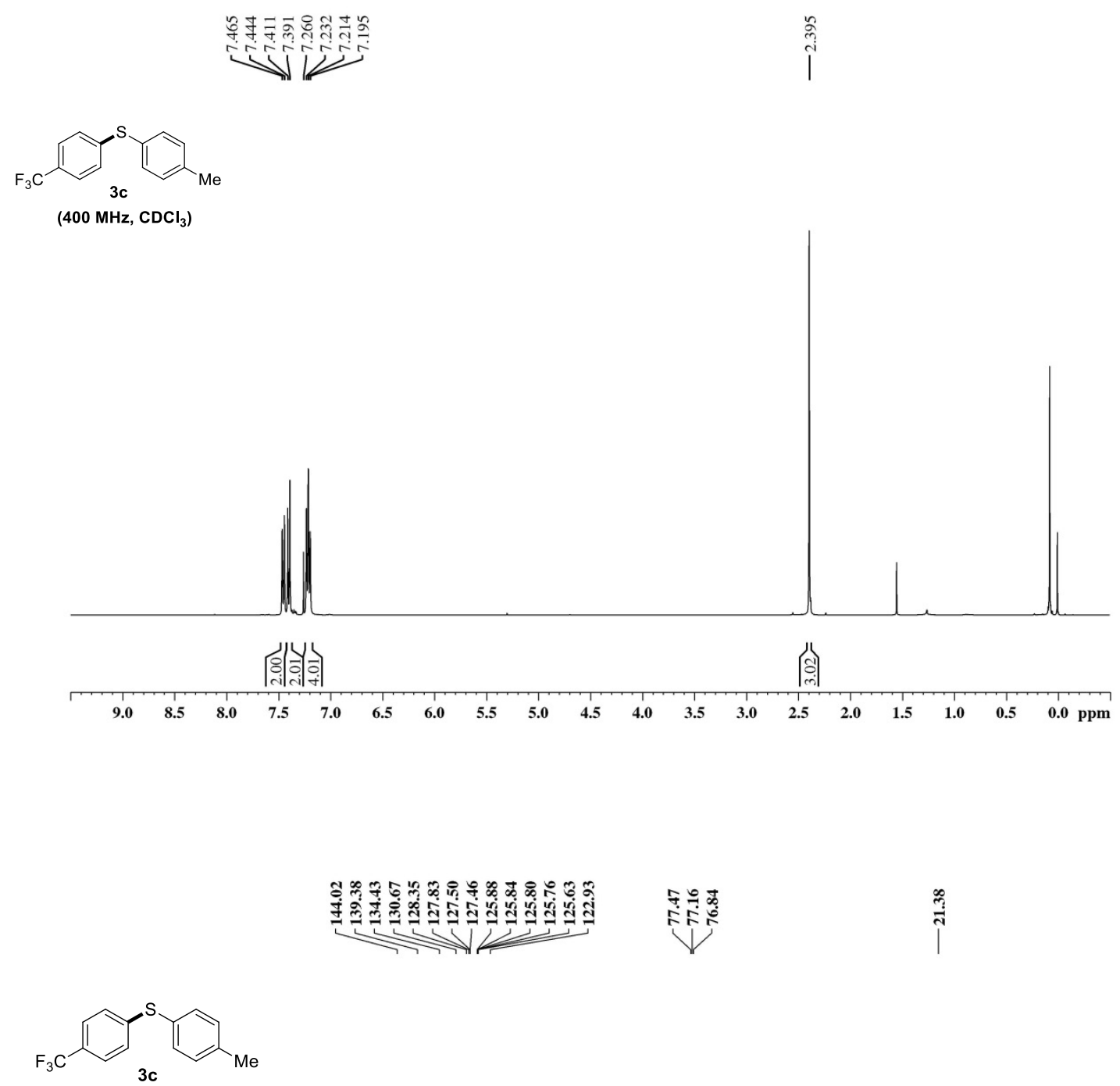

(100 MHz, $\mathrm{CDCl}_{3}$ )

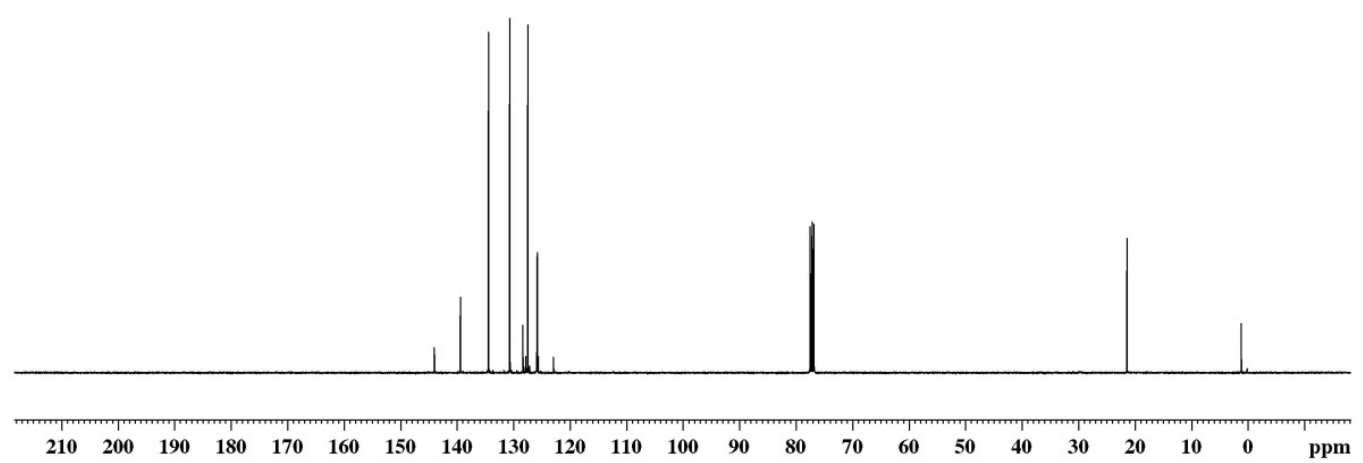




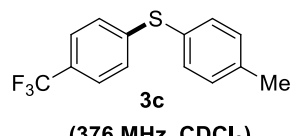

(376 MHz, $\mathrm{CDCl}_{3}$ )

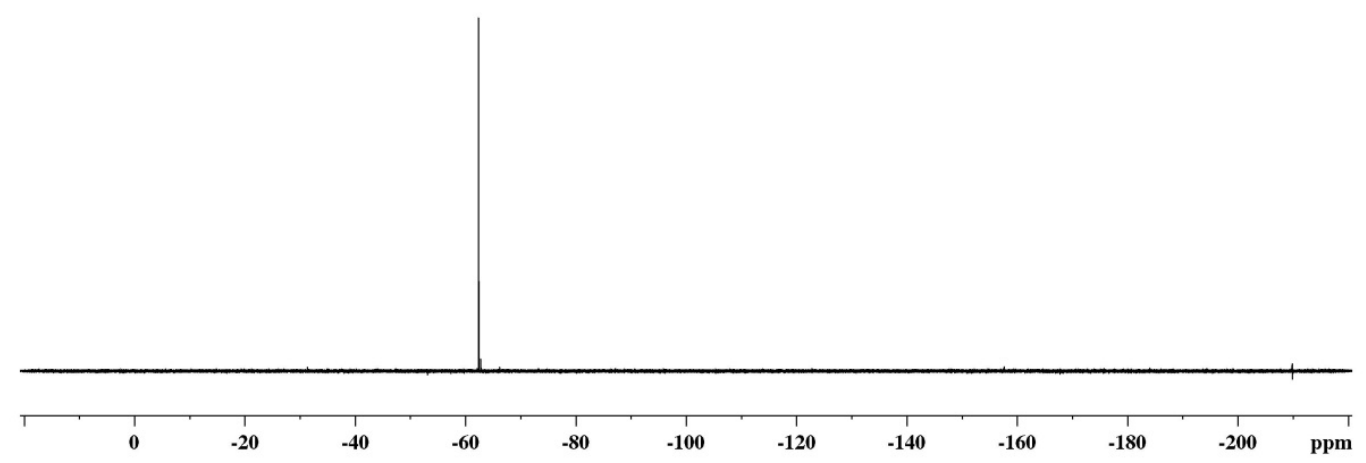



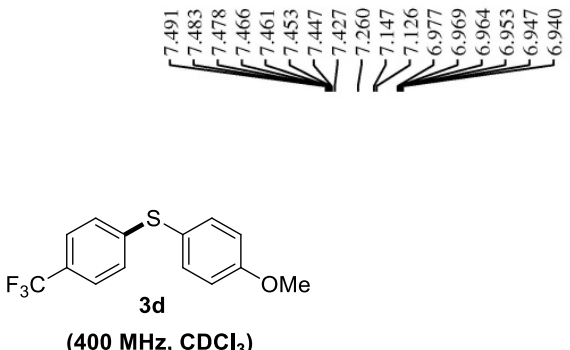

$\left(400 \mathrm{MHz}, \mathrm{CDCl}_{3}\right)$

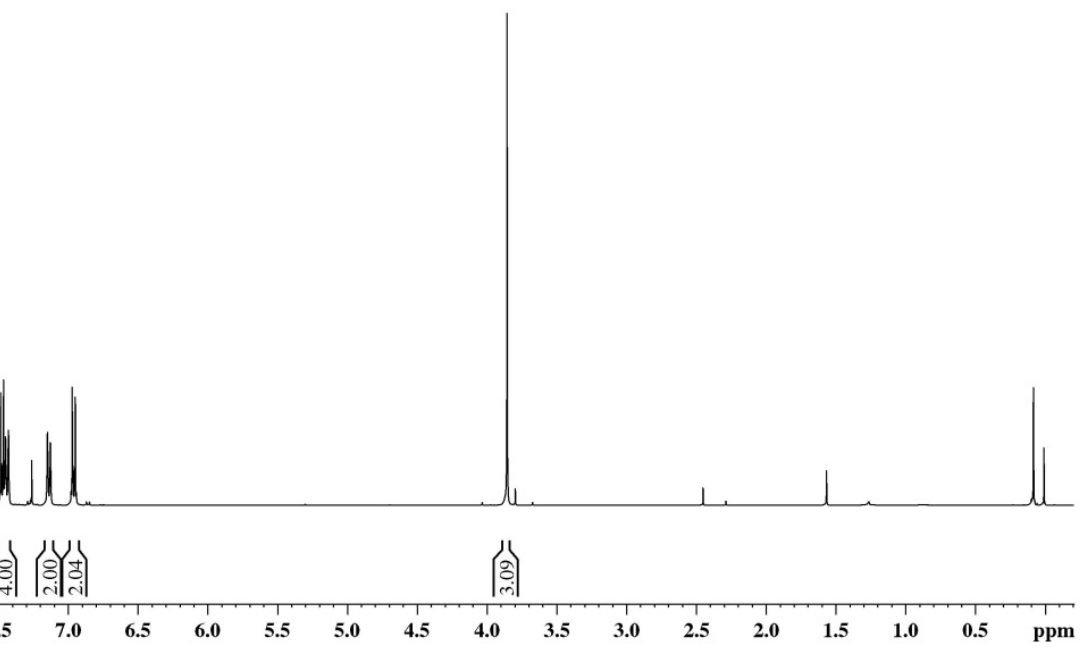

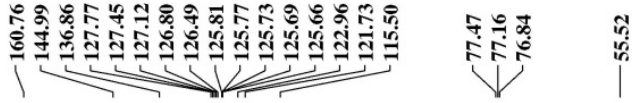

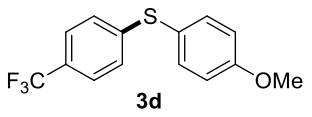

$\left(100 \mathrm{MHz}, \mathrm{CDCl}_{3}\right)$

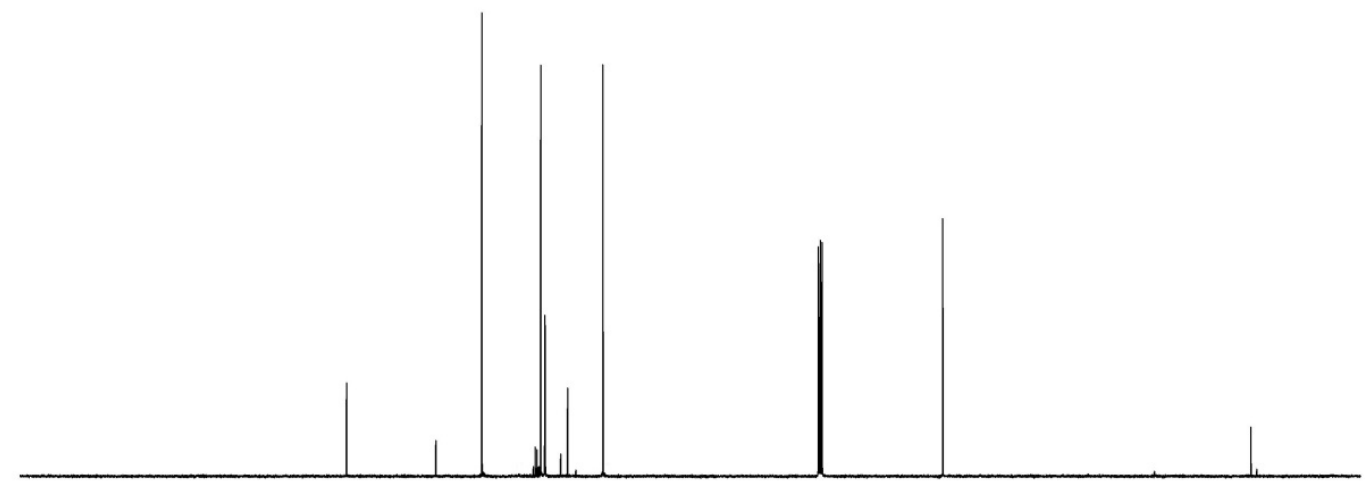

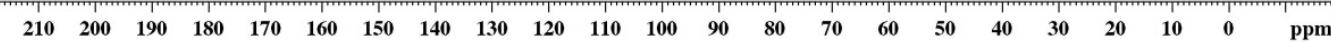




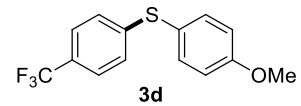

(376 MHz, $\mathrm{CDCl}_{3}$ )

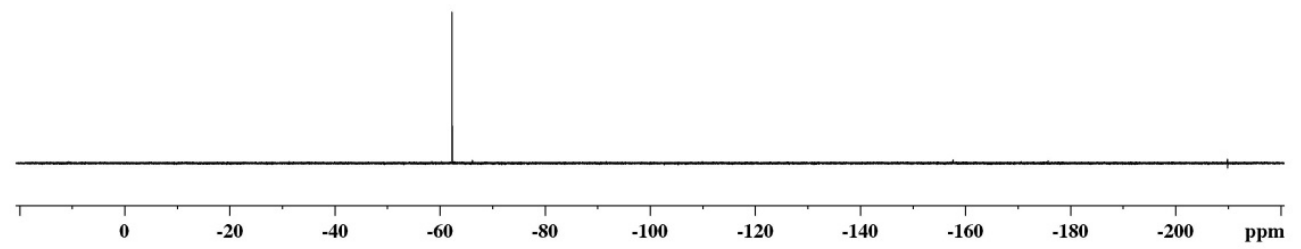



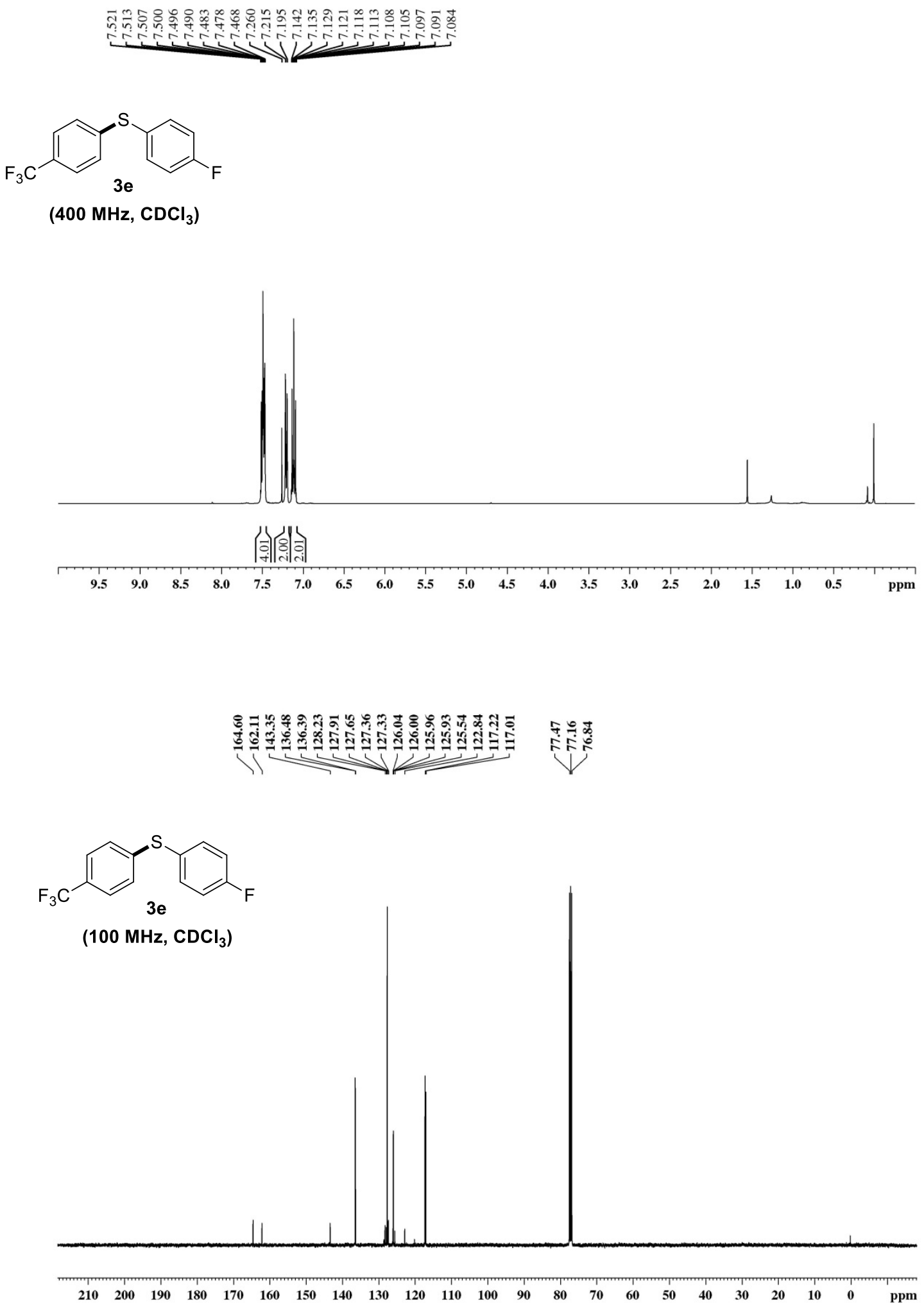

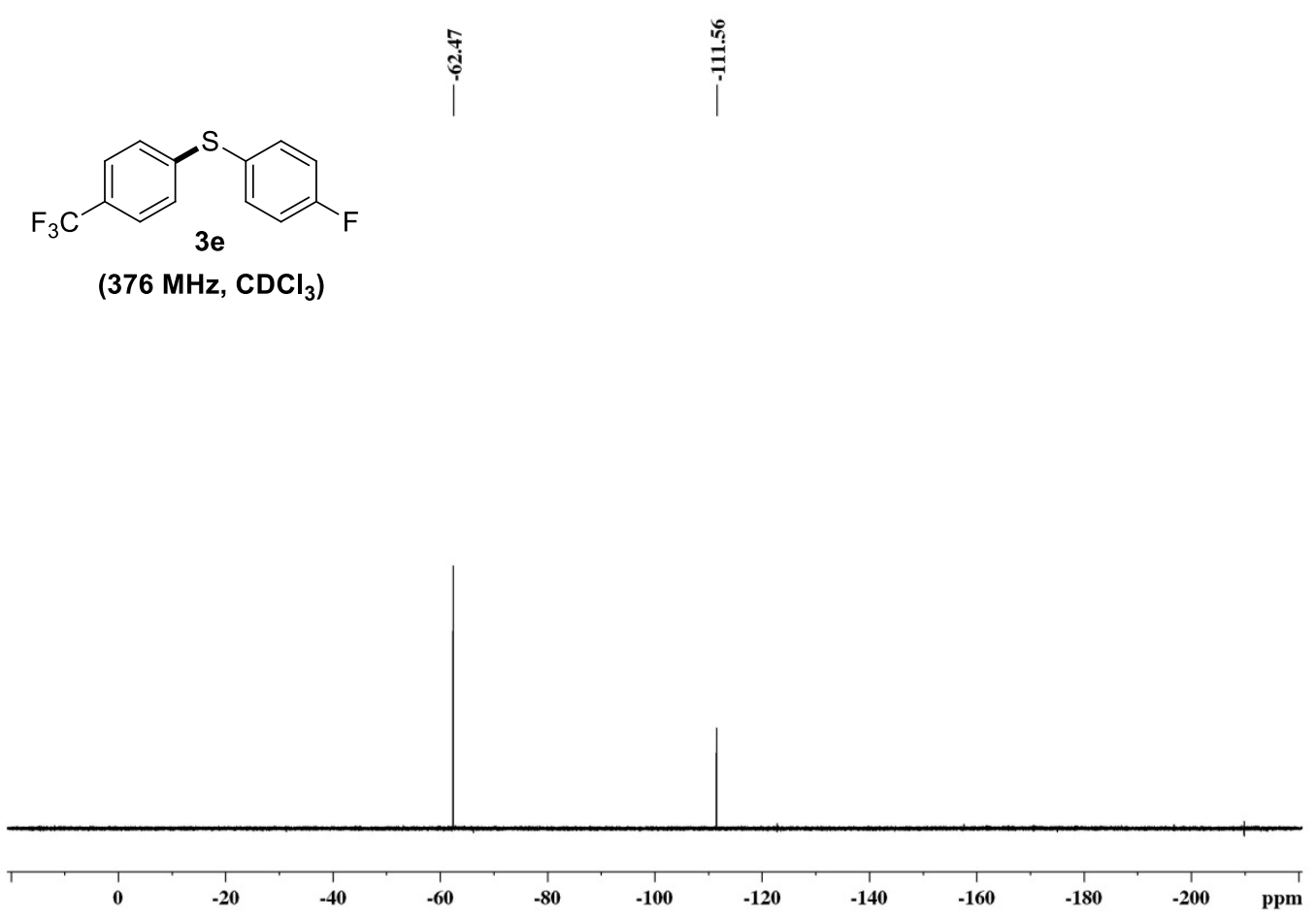

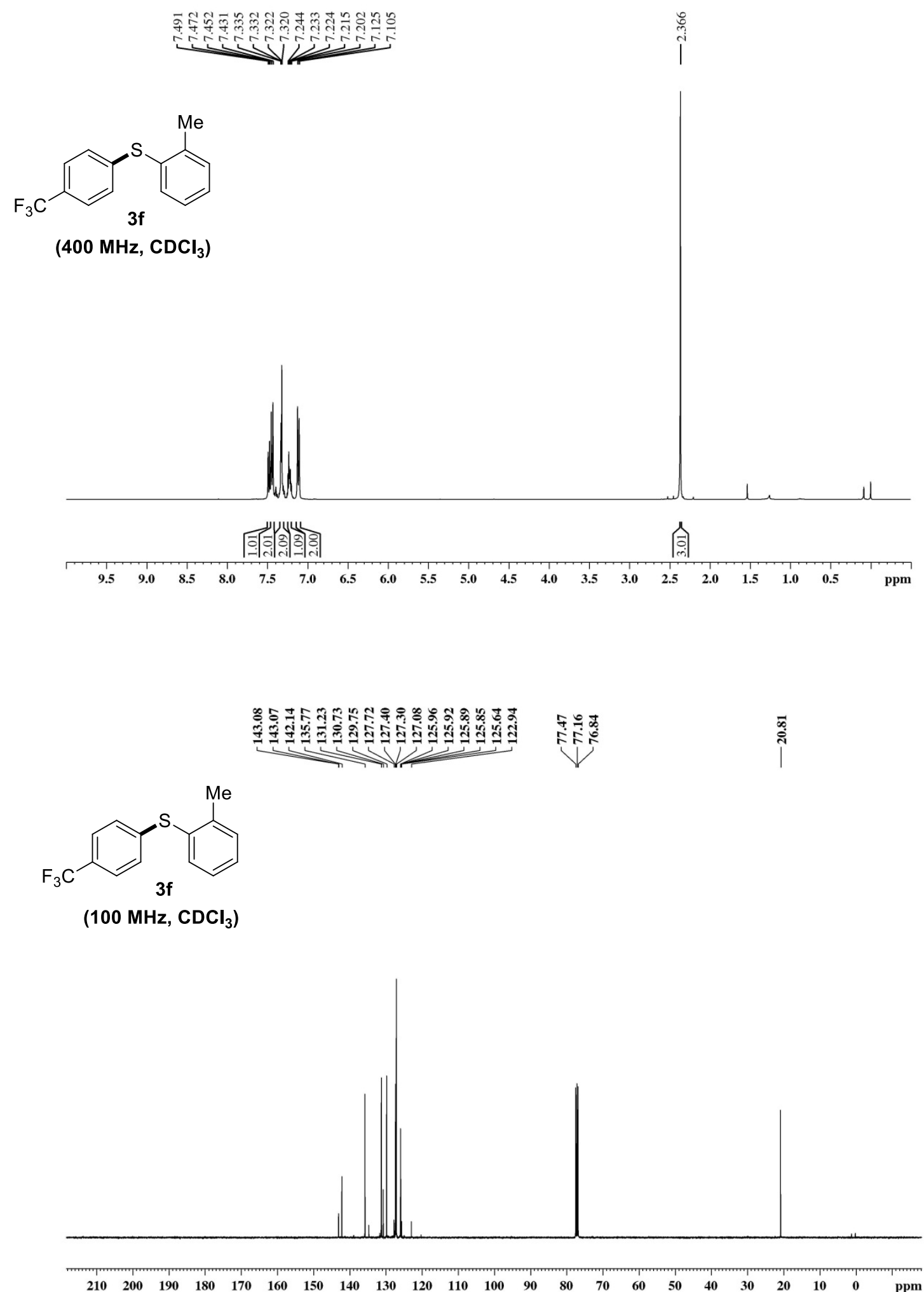

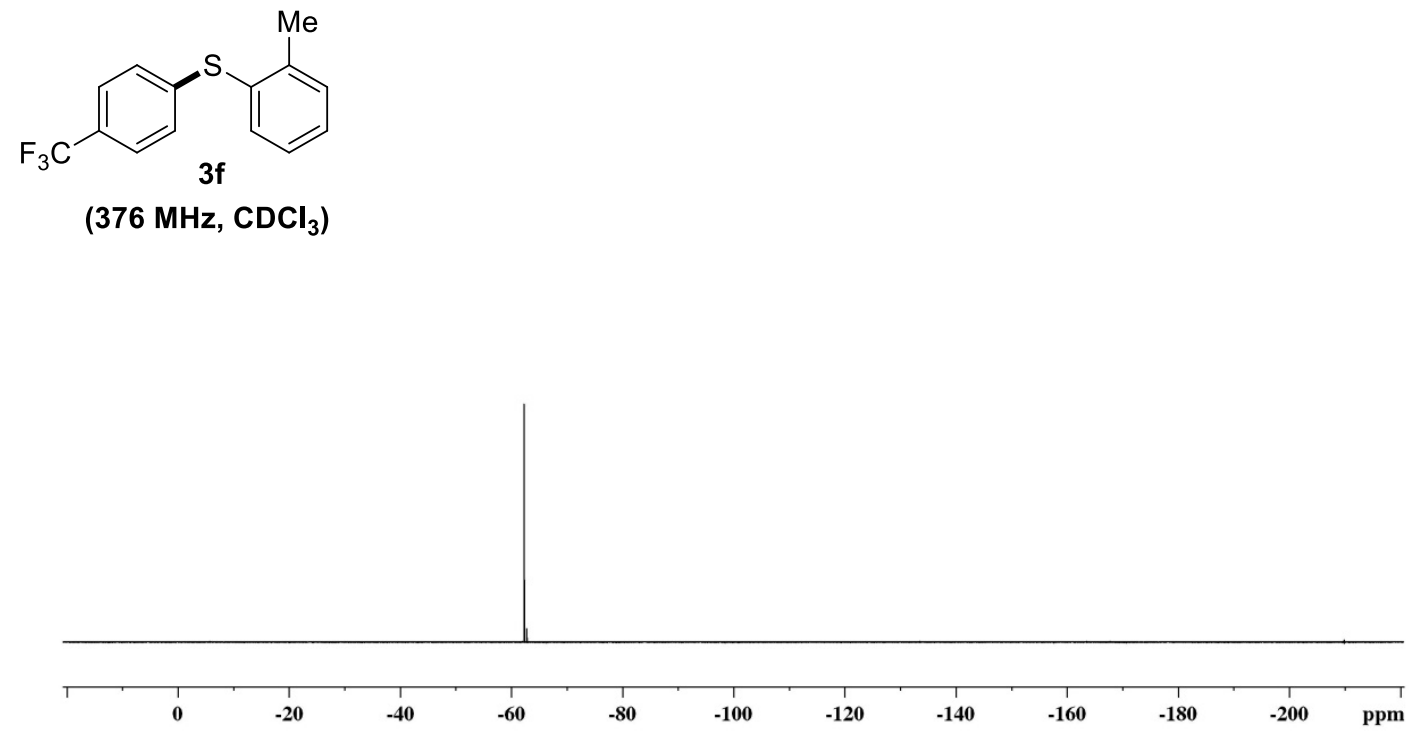
(1)initin

C.11

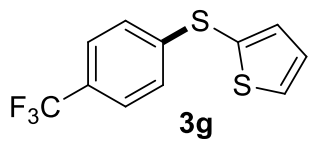

(400 $\mathrm{MHz}, \mathrm{CDCl}_{3}$ )
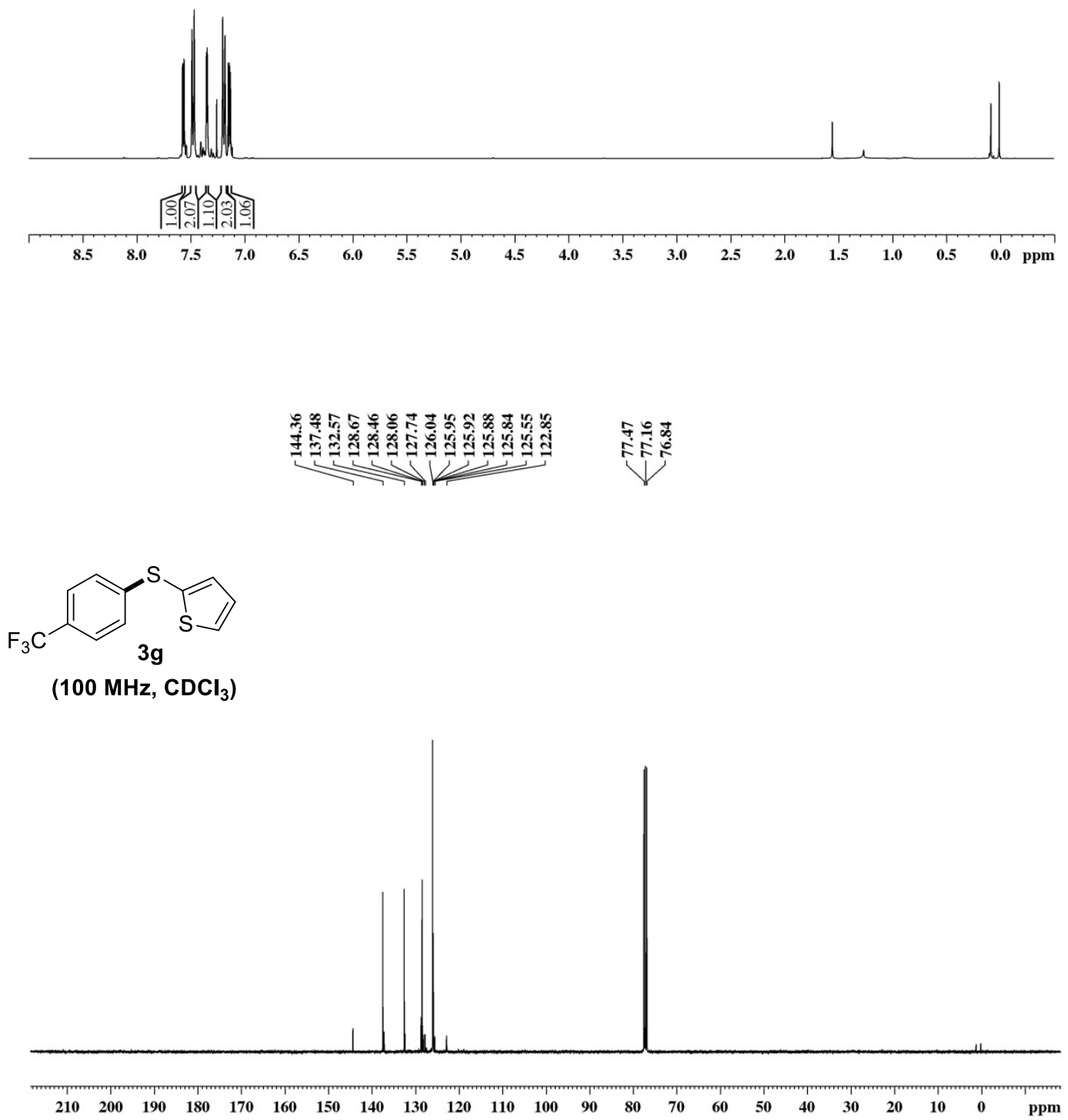

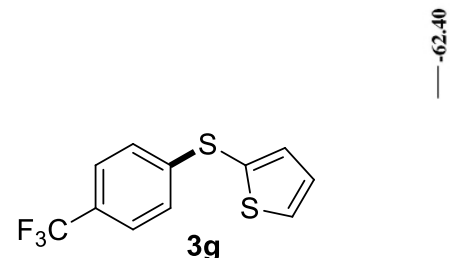

(376 $\mathrm{MHz}, \mathrm{CDCl}_{3}$ )

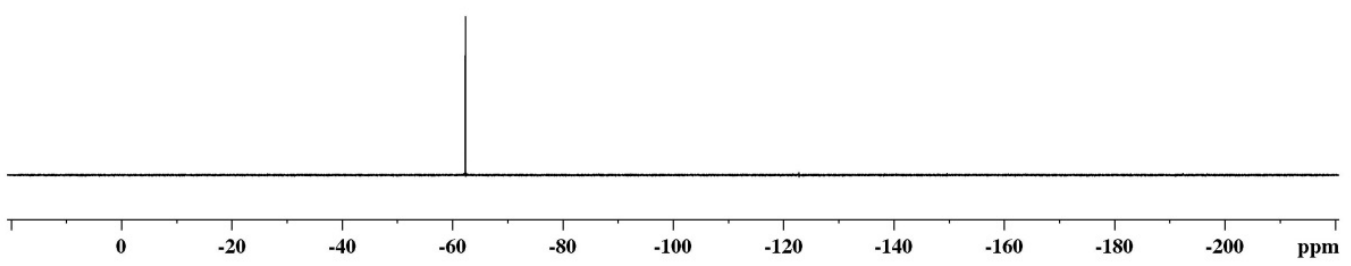



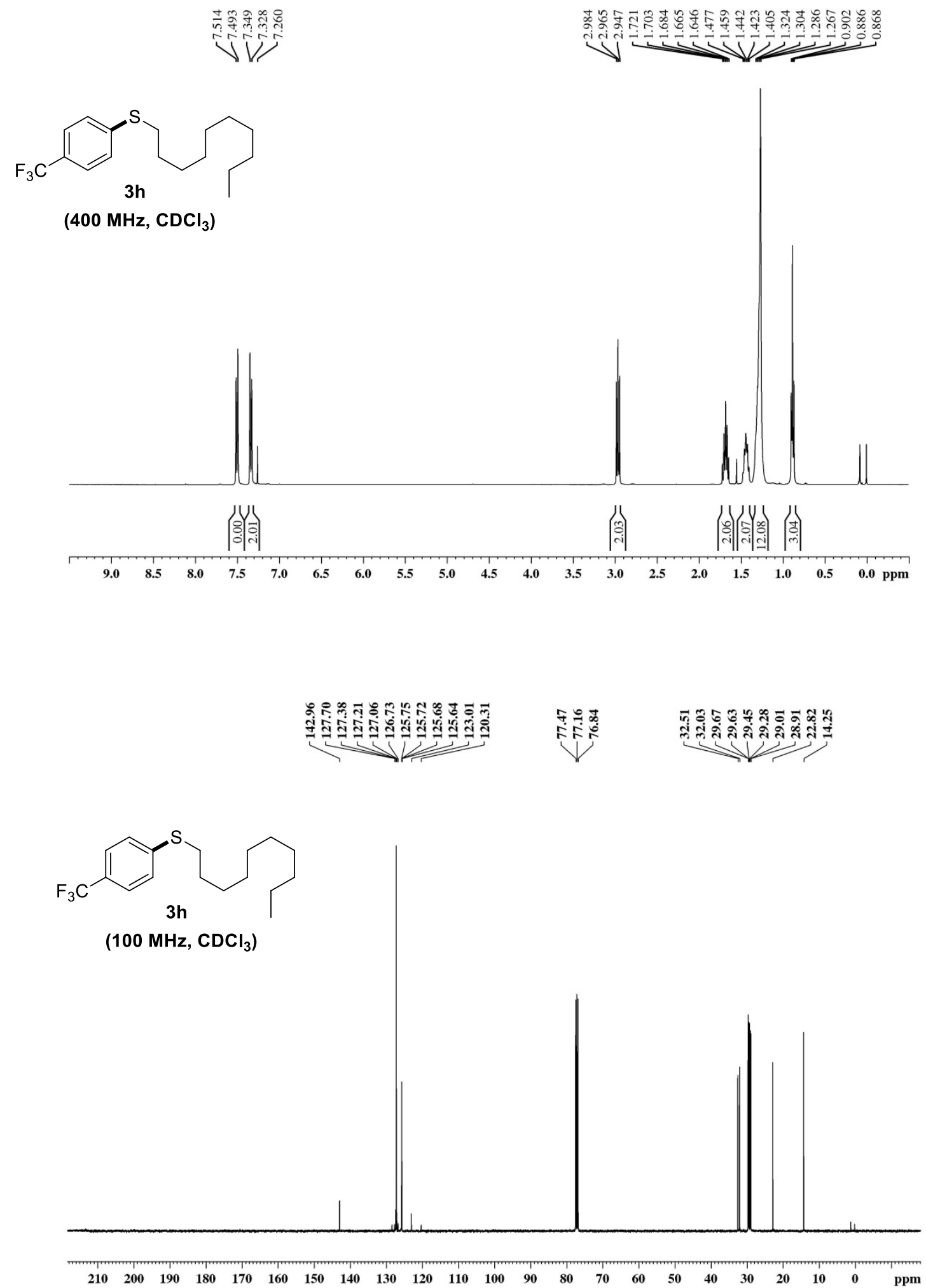


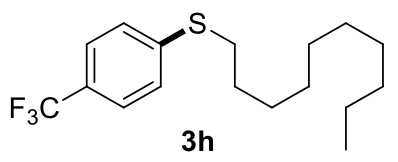

(376 $\mathrm{MHz} \mathrm{CDCl}_{3}$ )

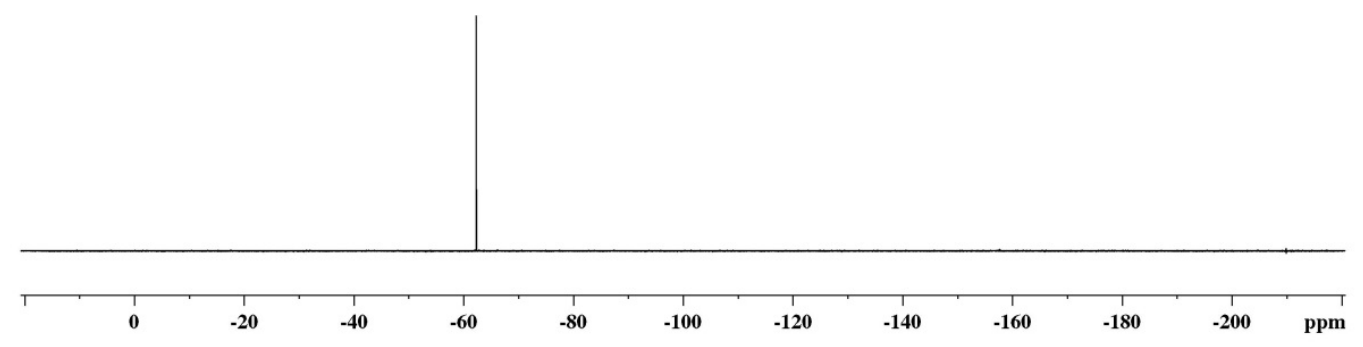




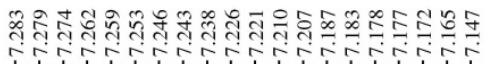

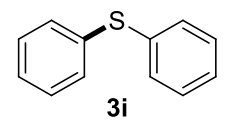

(400 $\mathrm{MHz}, \mathrm{CDCl}_{3}$ )

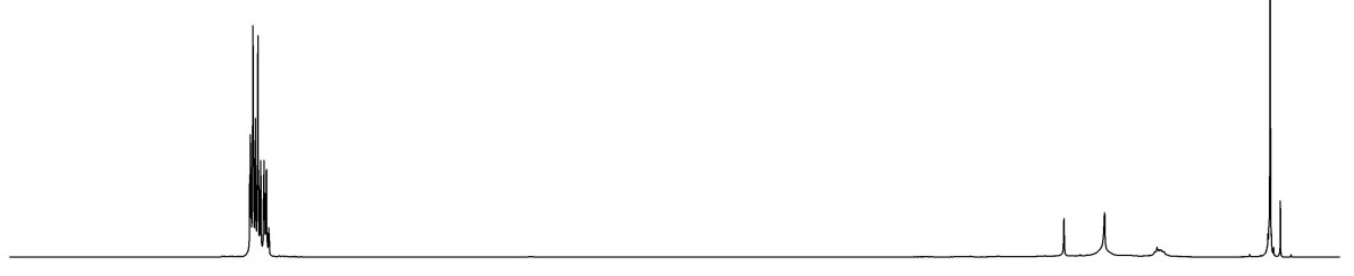

8 을

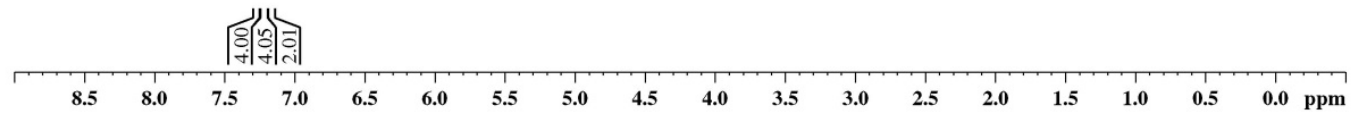

けำ

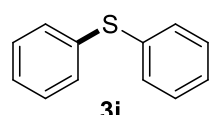

$3 \mathbf{i}$

(100 $\mathrm{MHz}, \mathrm{CDCl}_{3}$ )

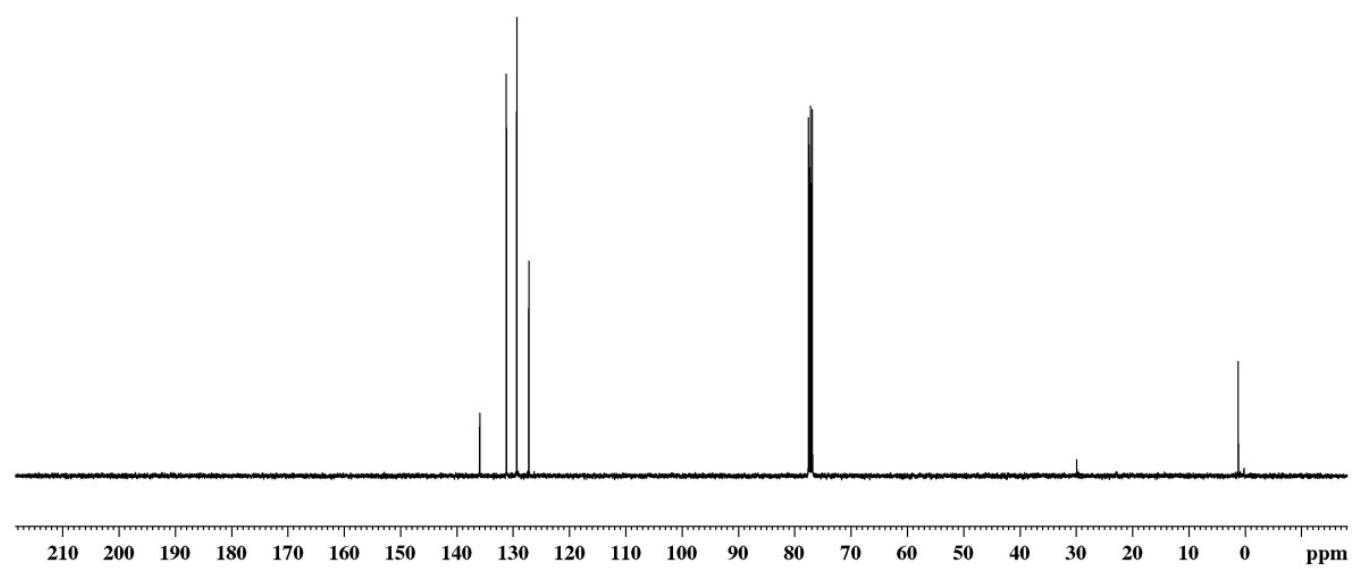



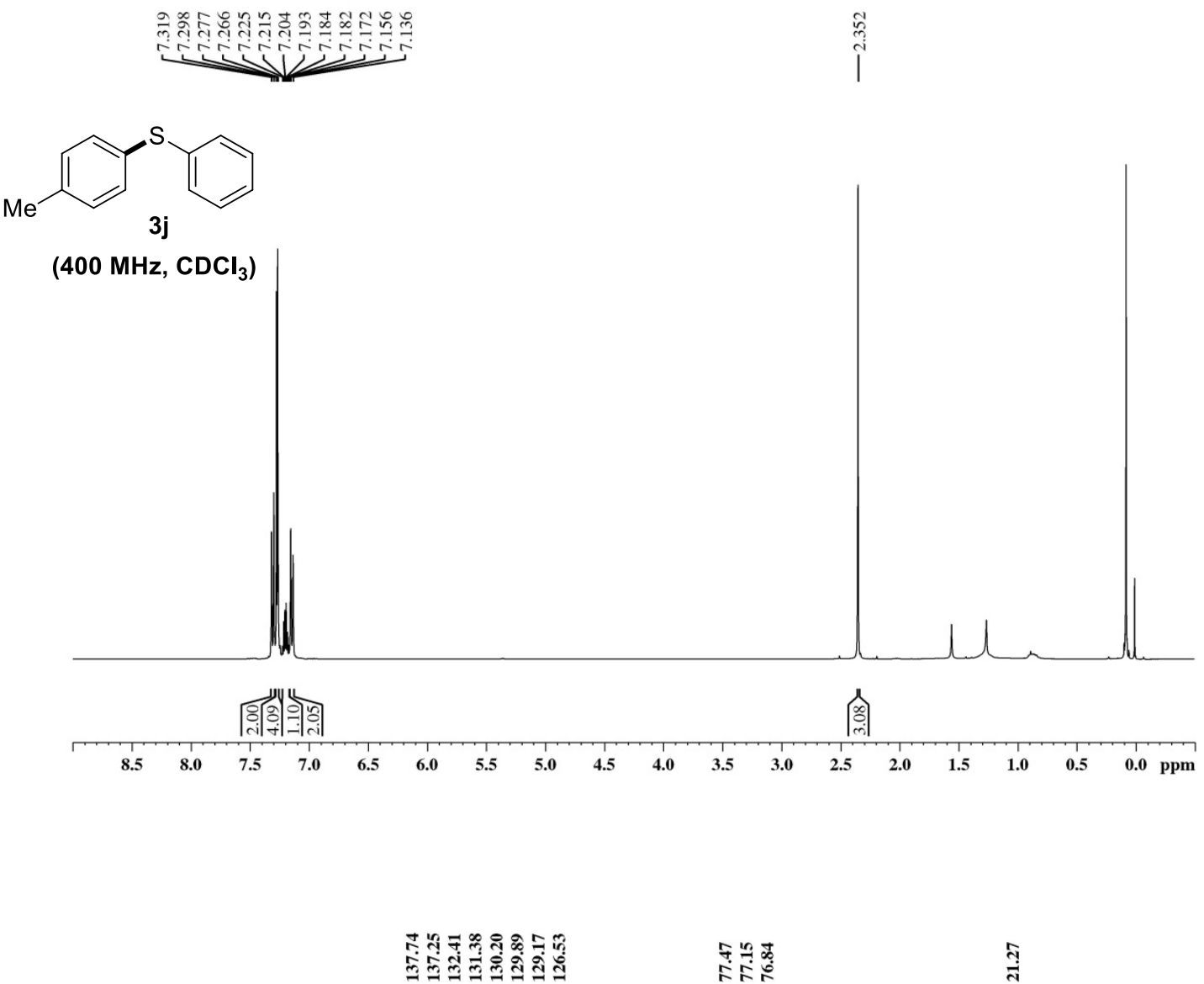

กิ

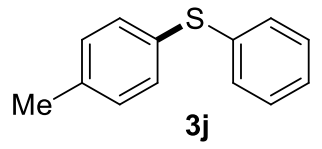

$\left.\left(100 \mathrm{MHz}^{\mathrm{CDCl}}\right)_{3}\right)$

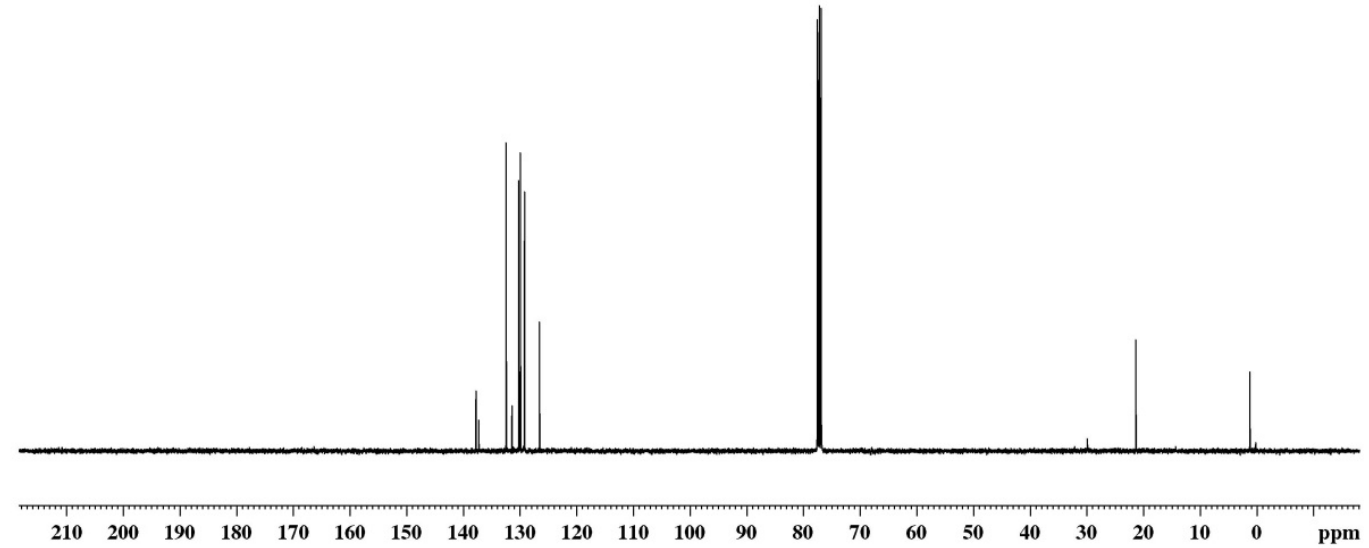




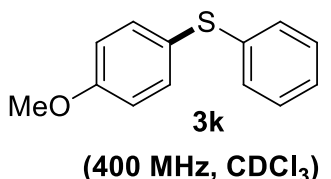

(400 MHz, $\mathrm{CDCl}_{3}$ )
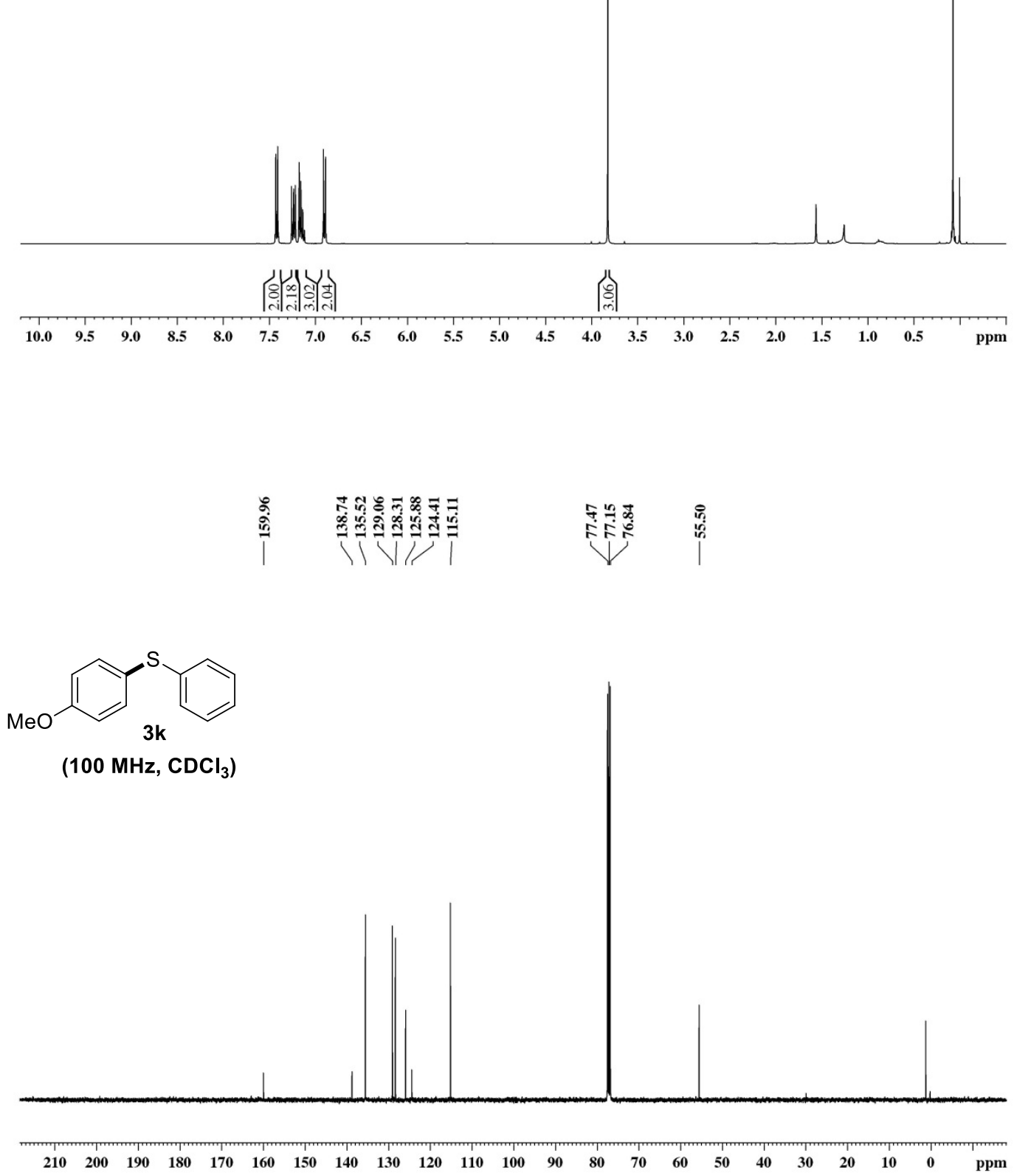

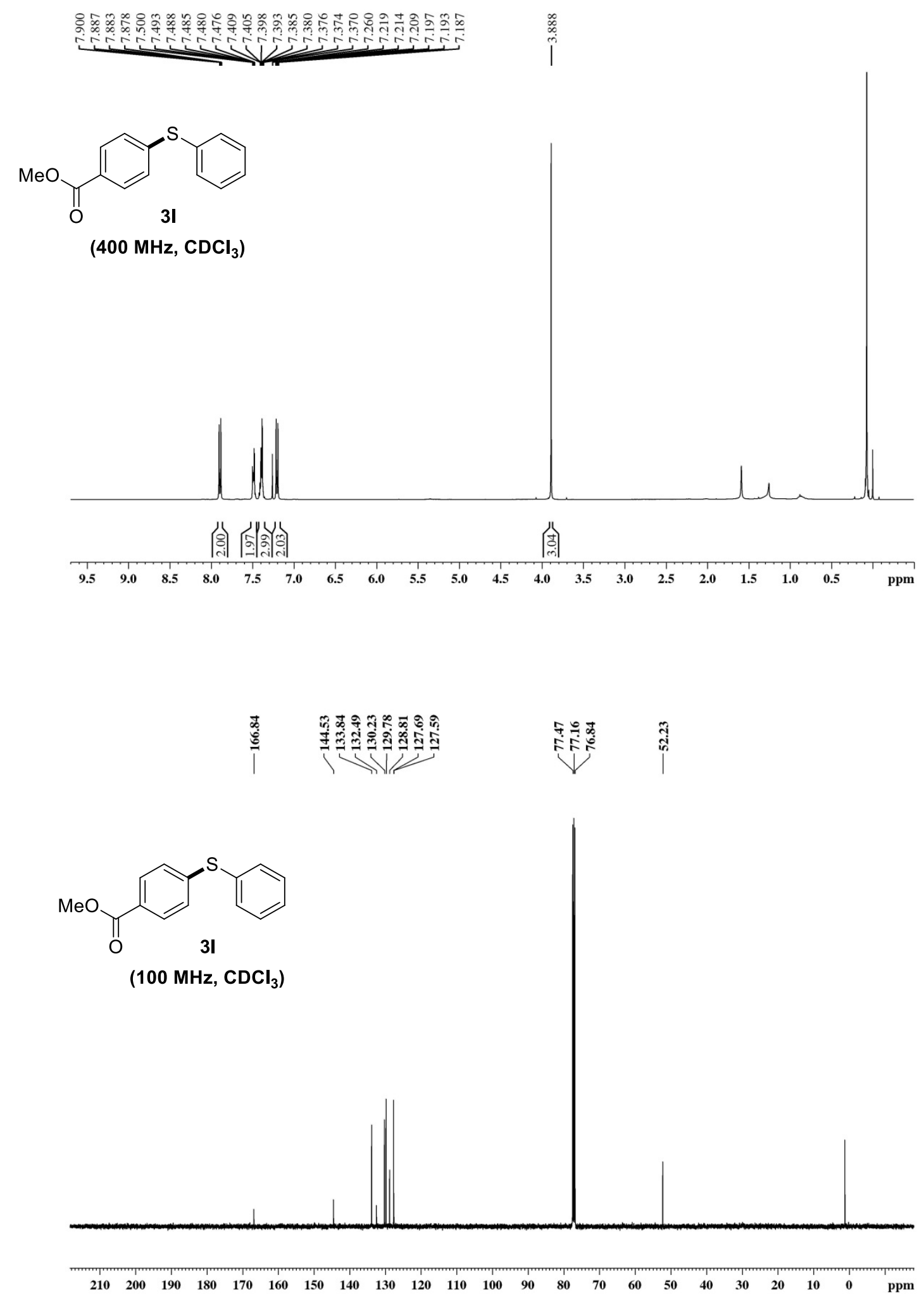

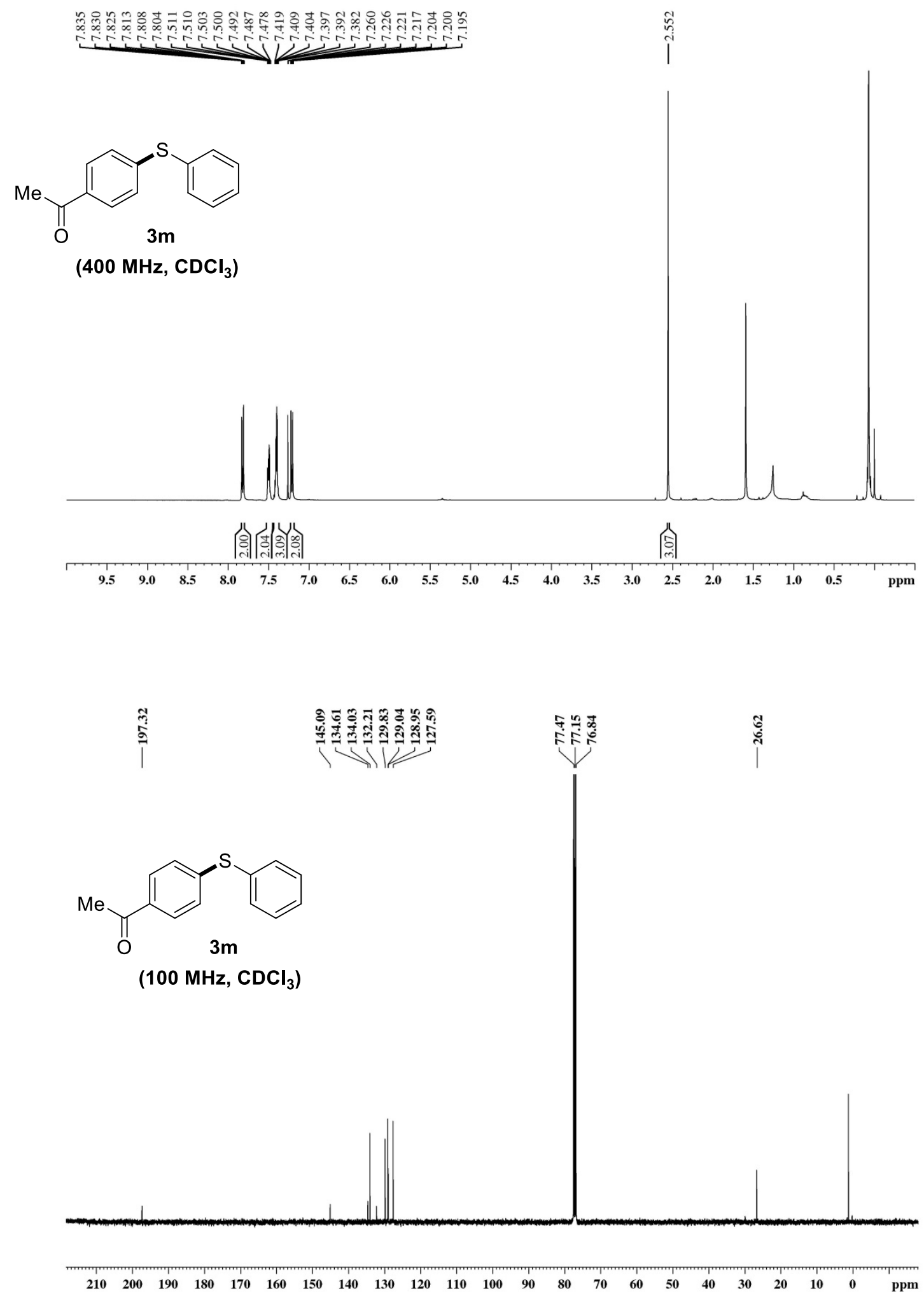


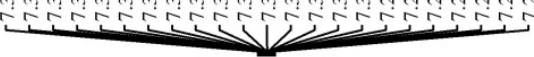
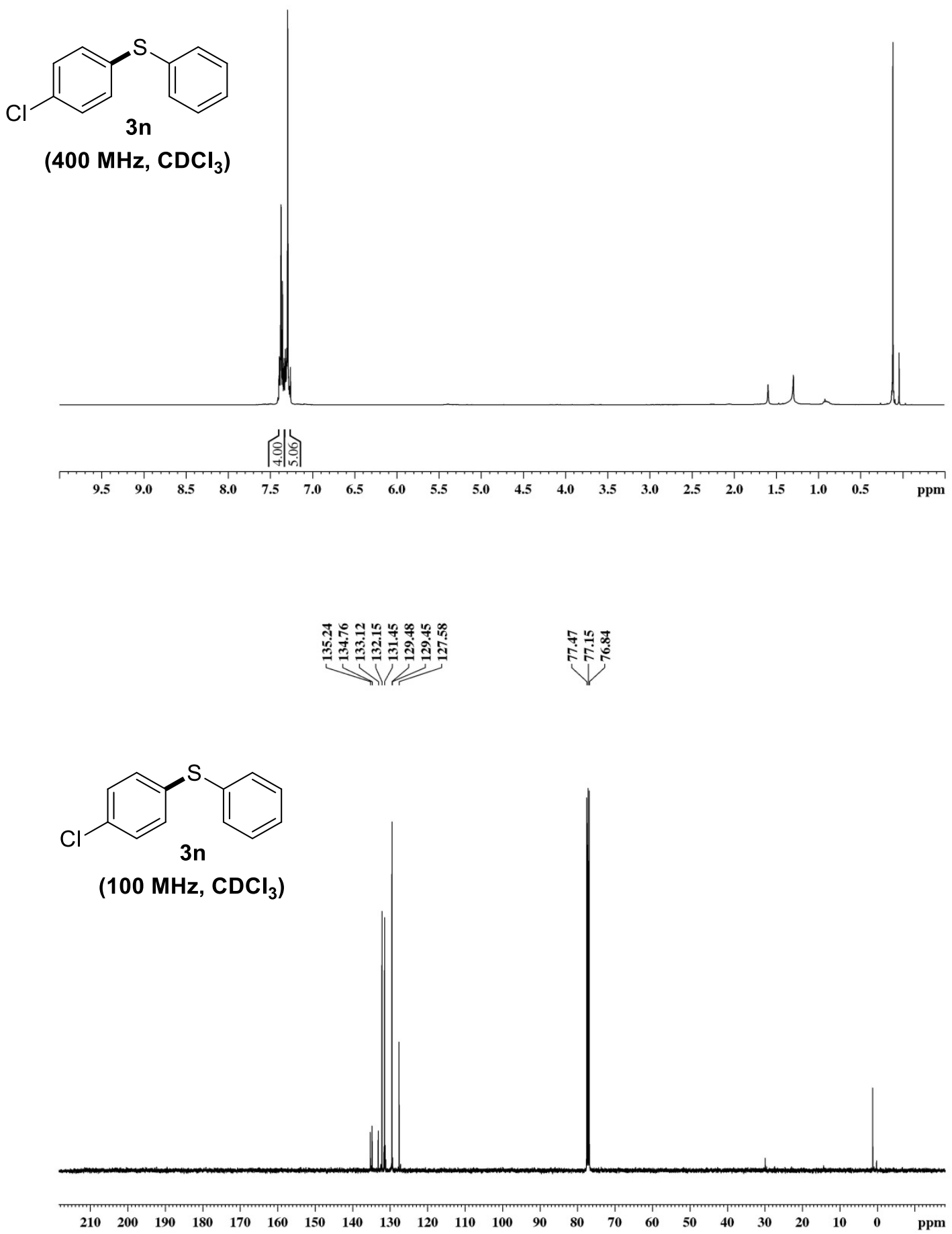

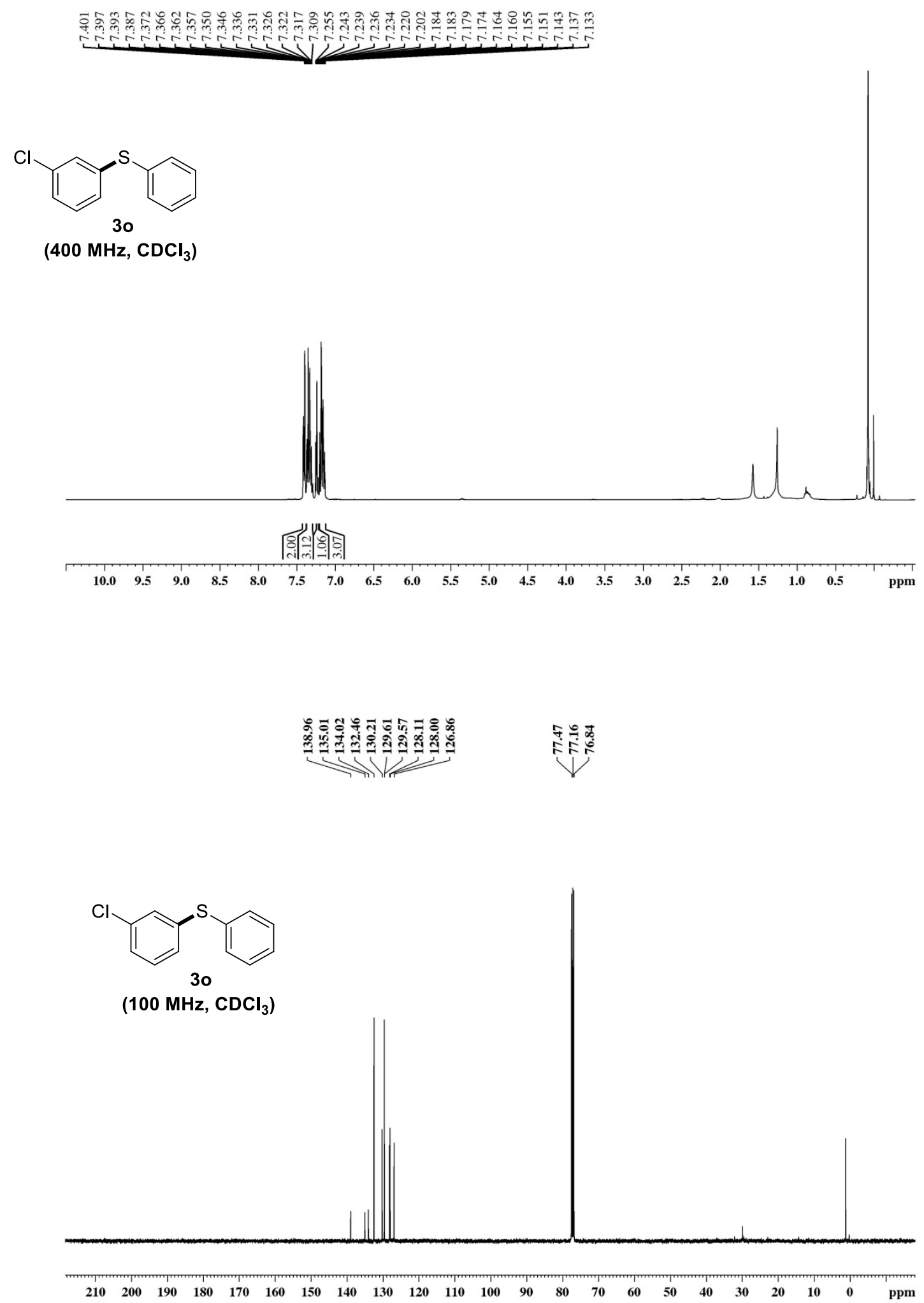
<smiles>Cc1ccccc1Sc1ccccc1</smiles>

(400 $\mathrm{MHz}, \mathrm{CDCl}_{3}$ )

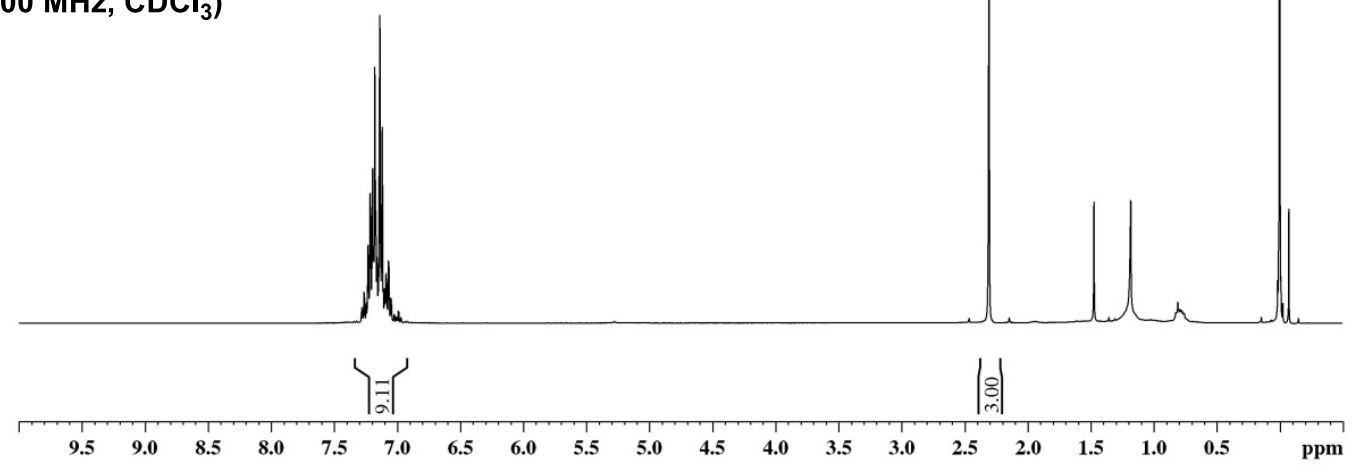<smiles>Cc1ccccc1Sc1ccccc1</smiles>

(100 MHz, $\mathrm{CDCl}_{3}$ )
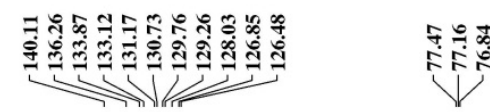

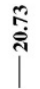
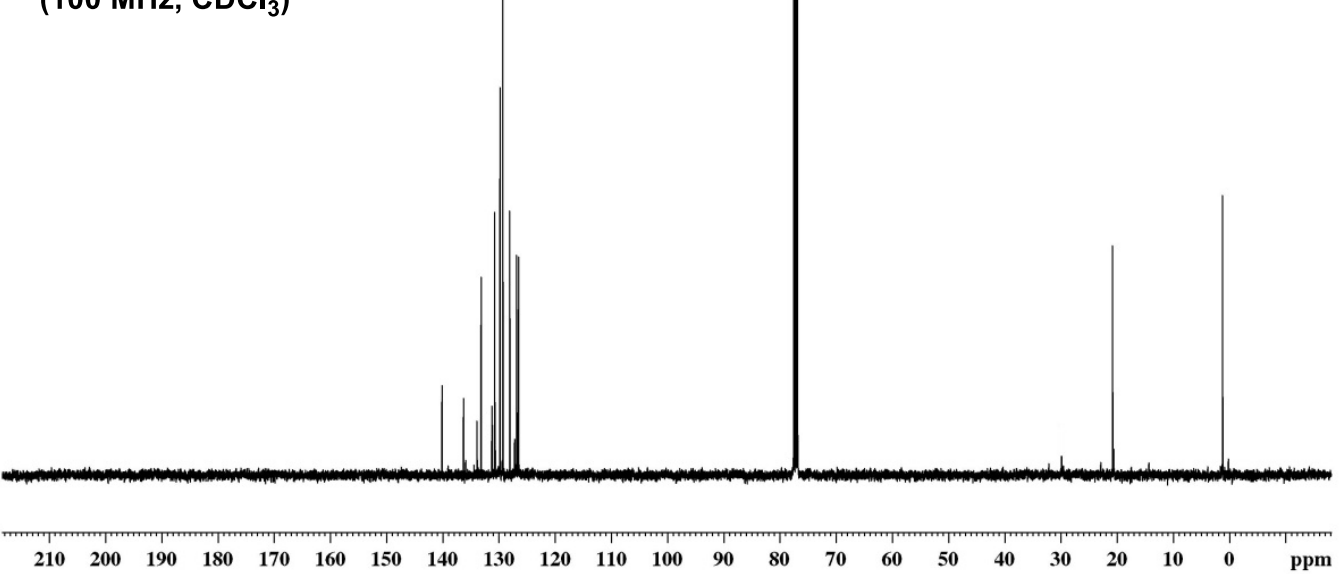

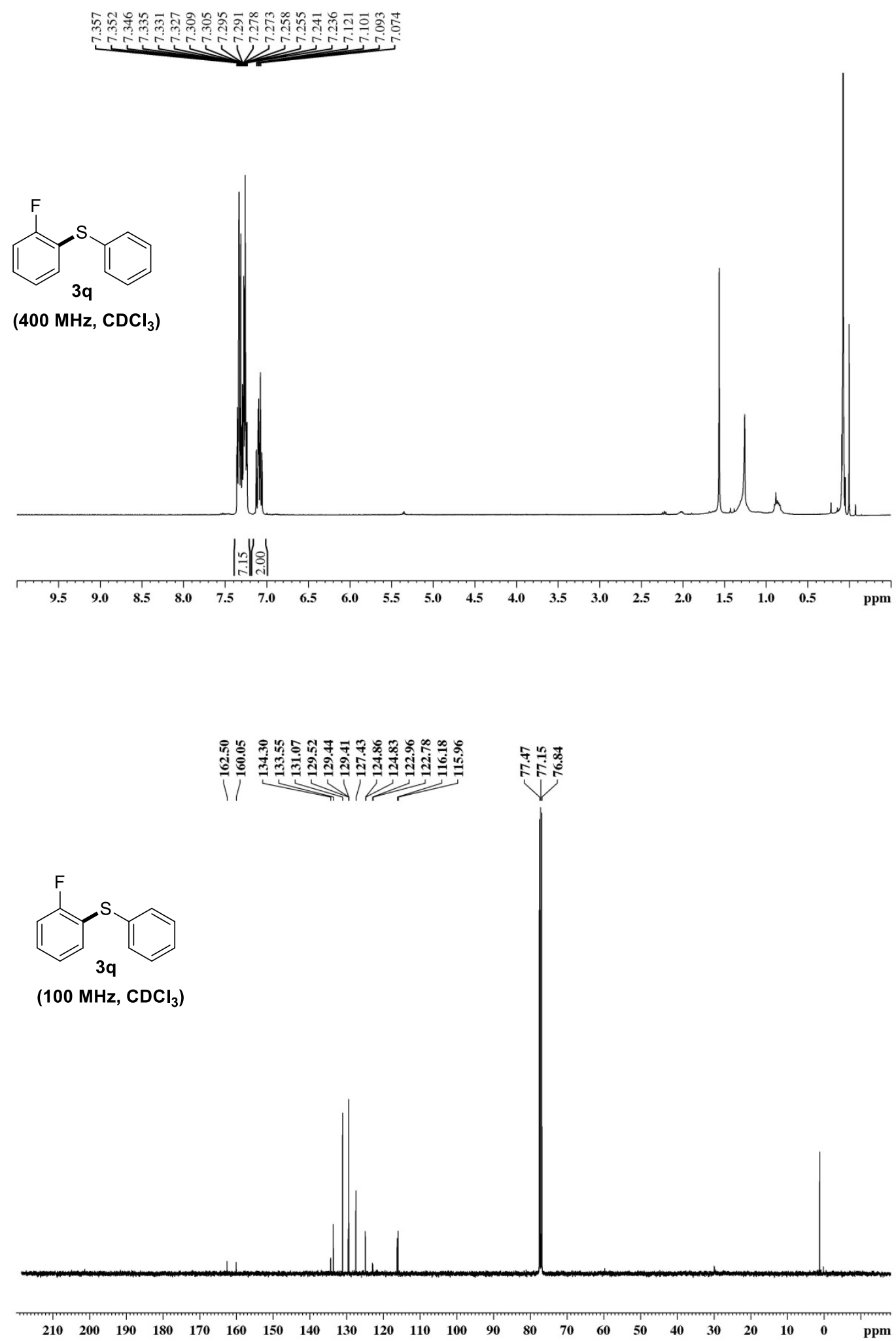


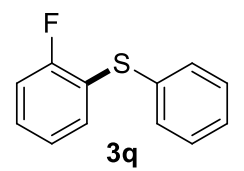

(376 $\mathrm{MHz}, \mathrm{CDCl}_{3}$ )

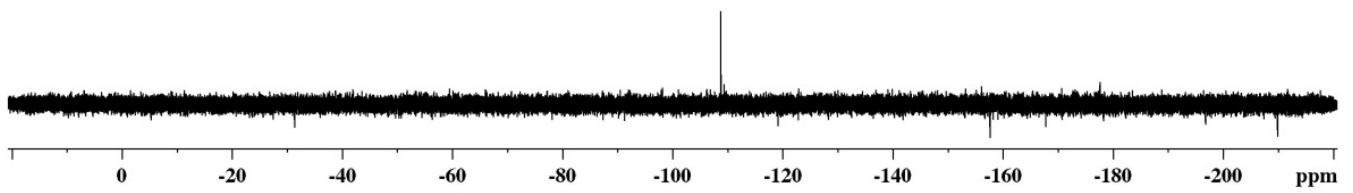



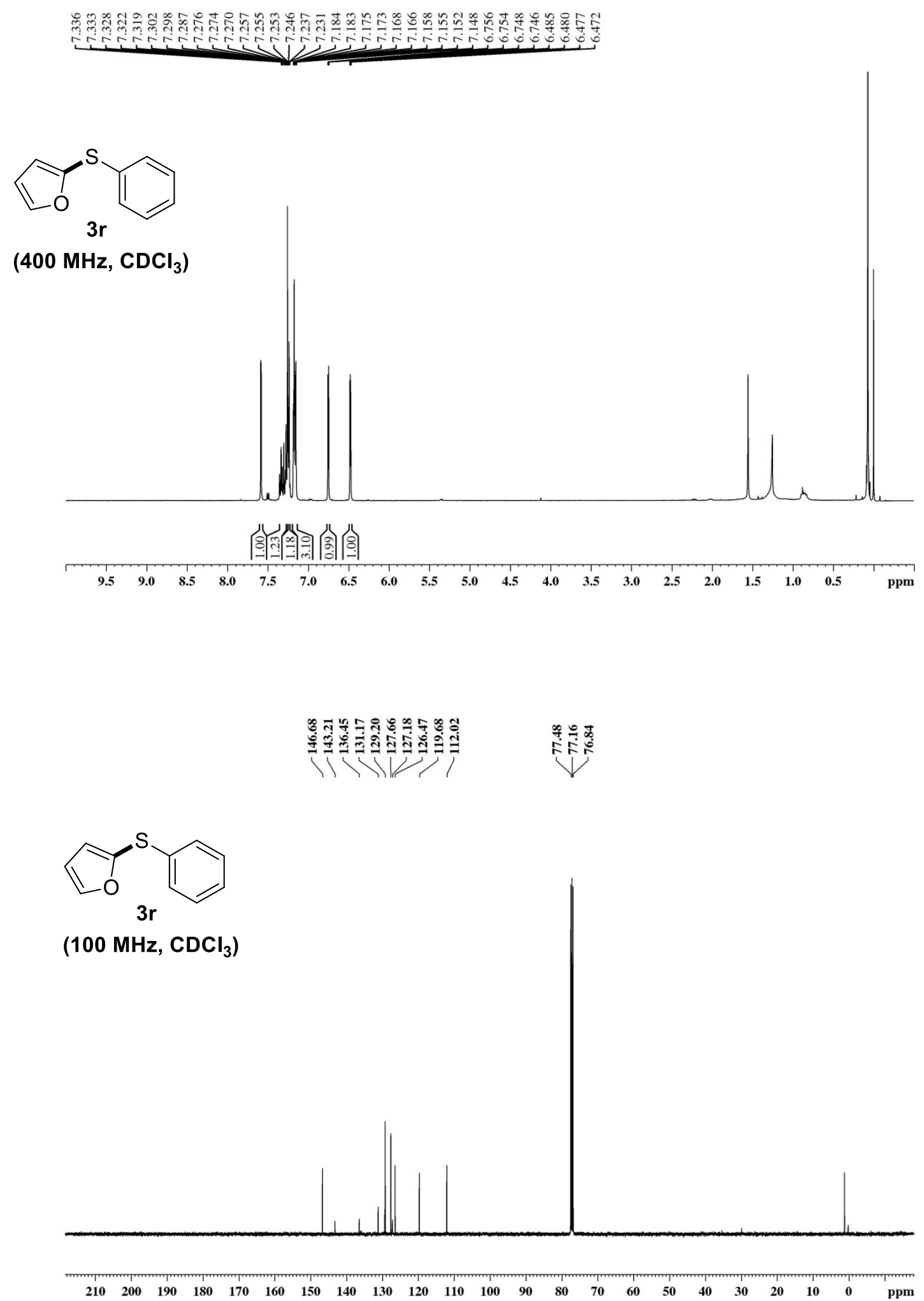

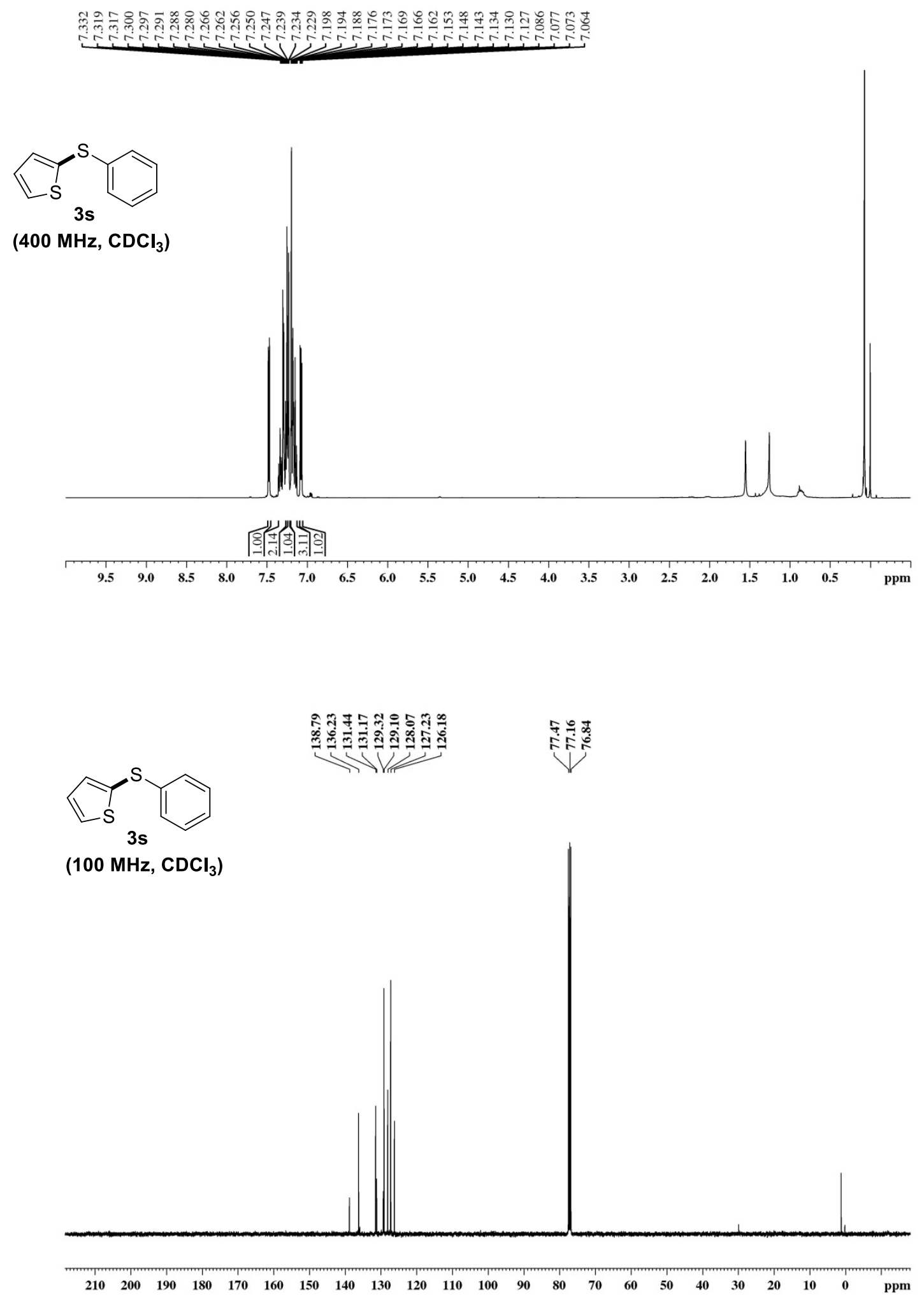

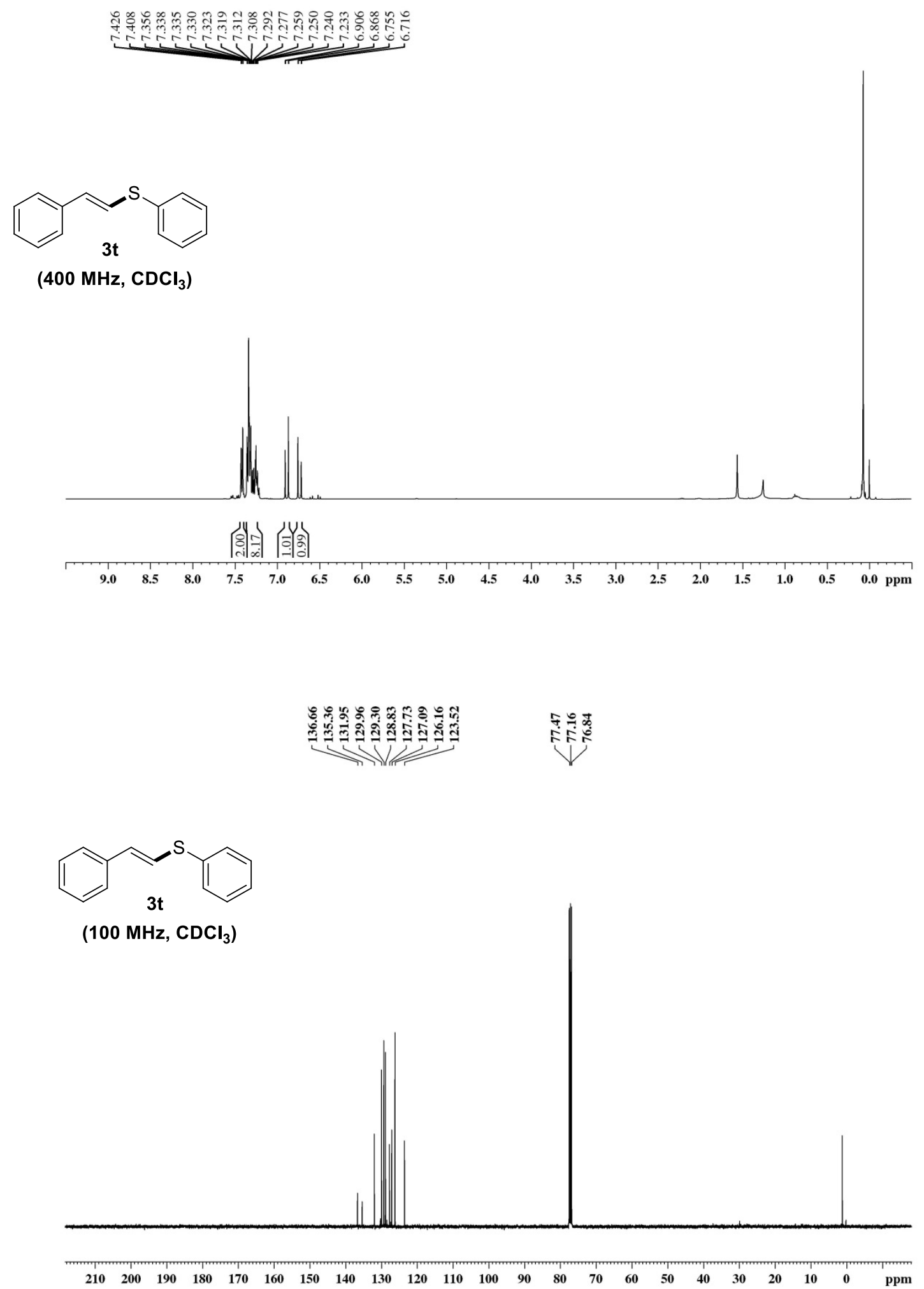

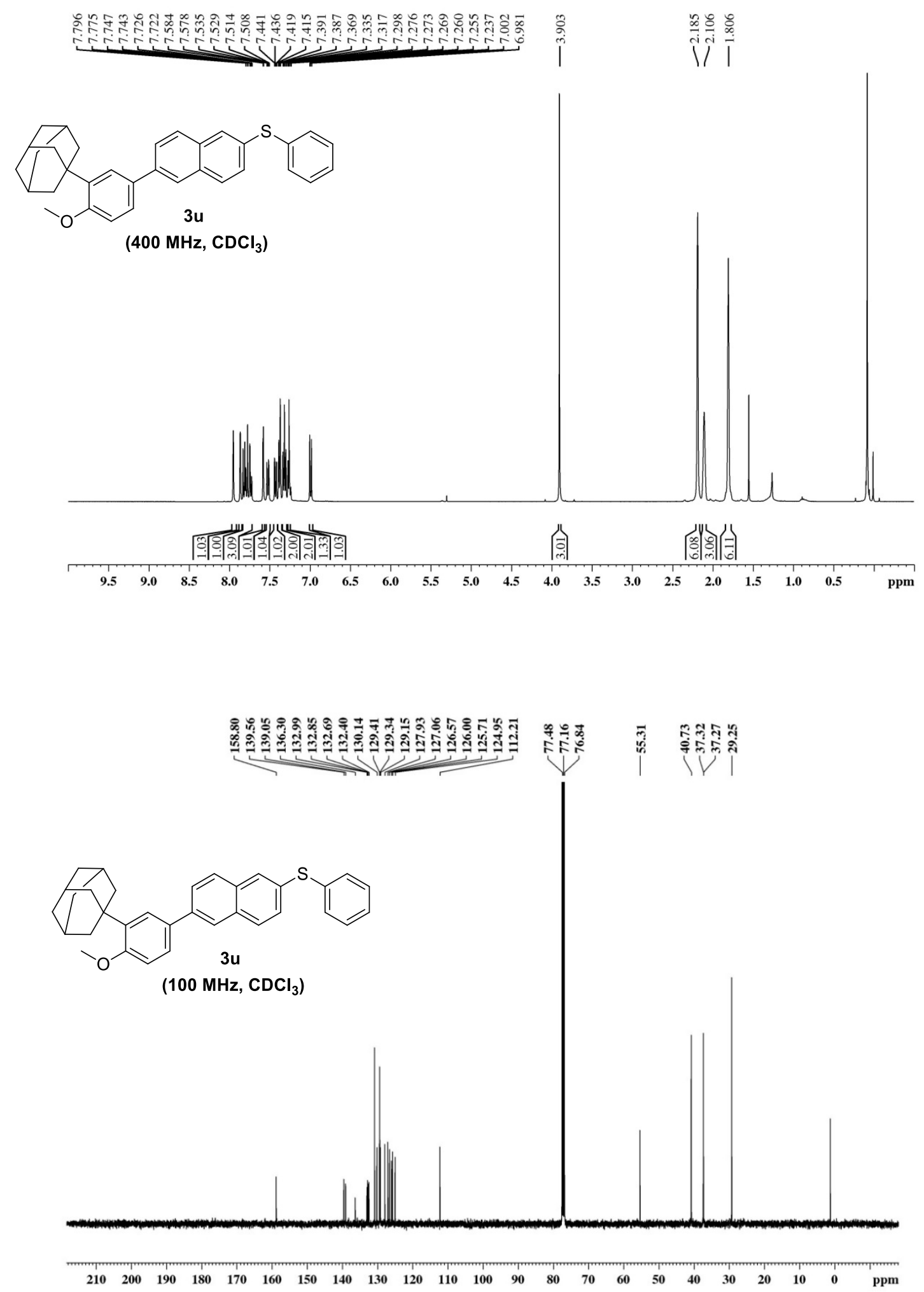

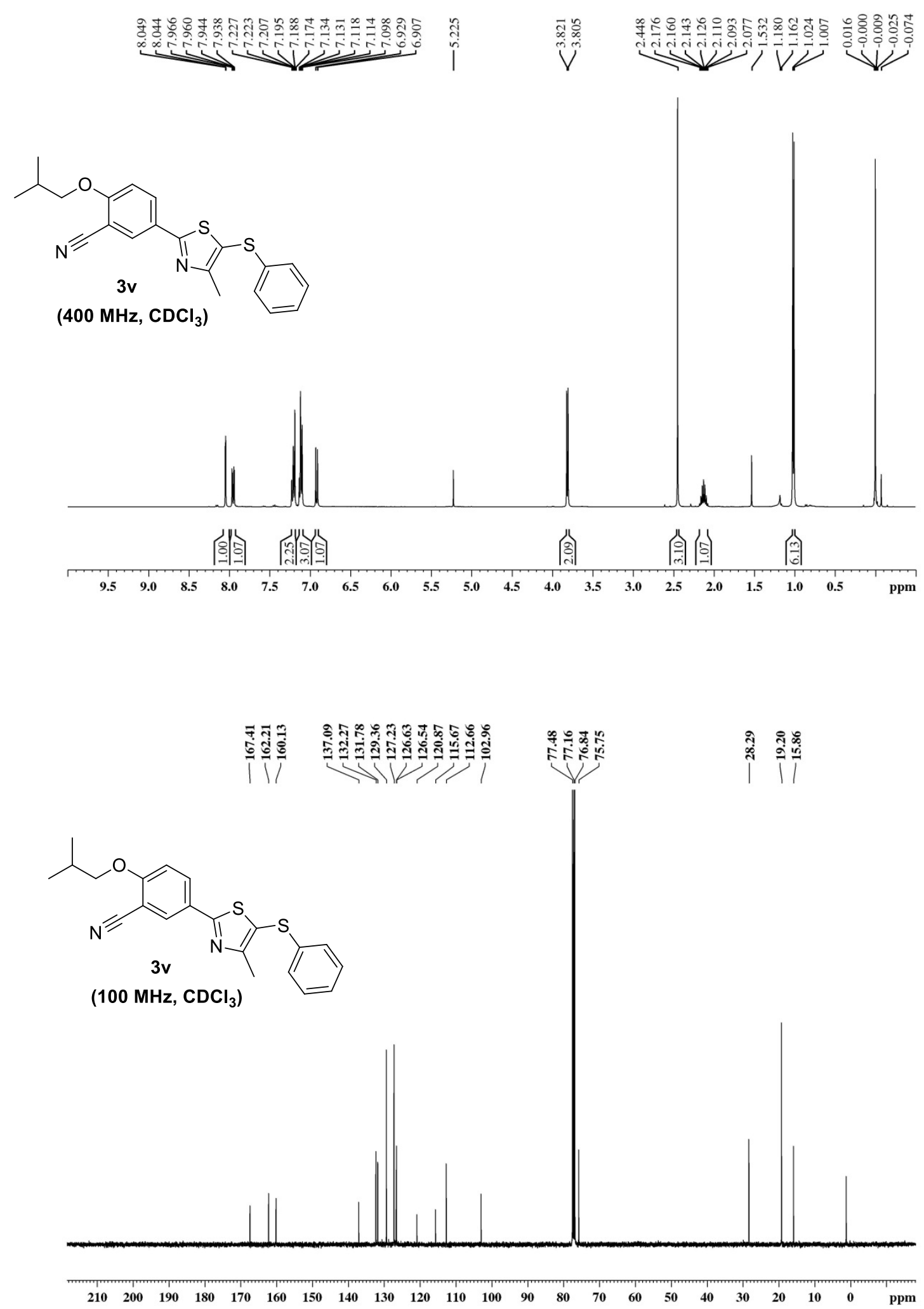

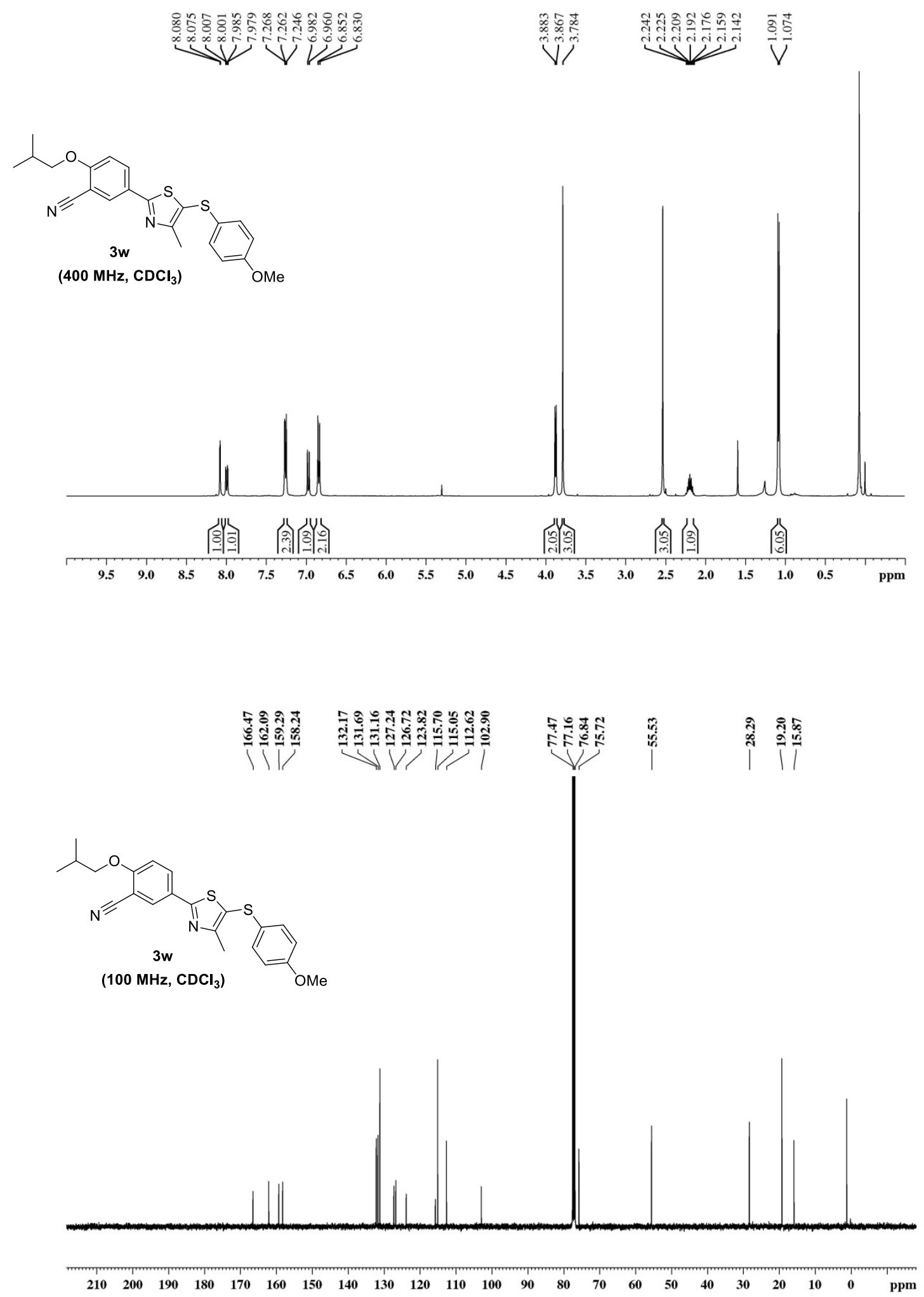

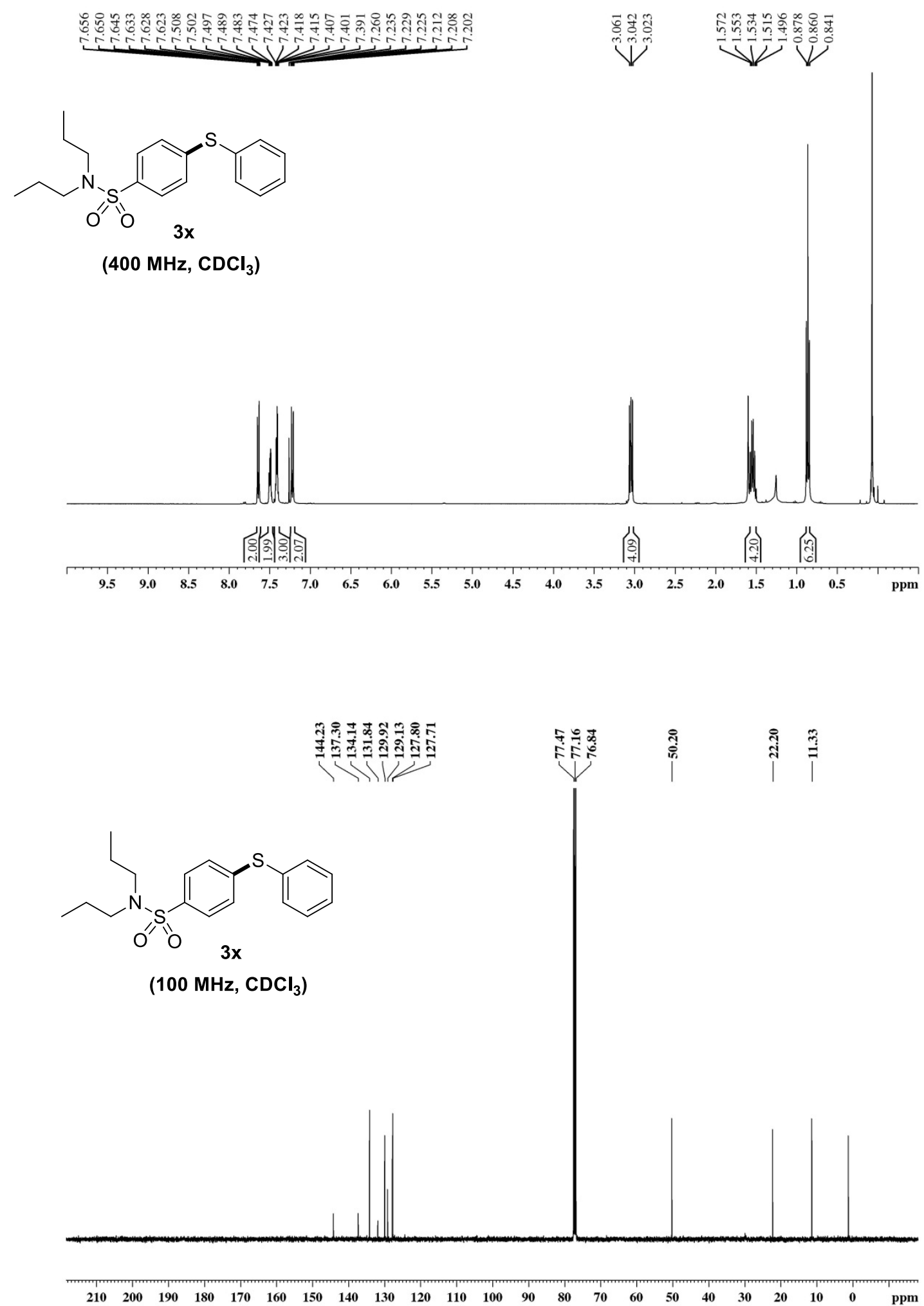

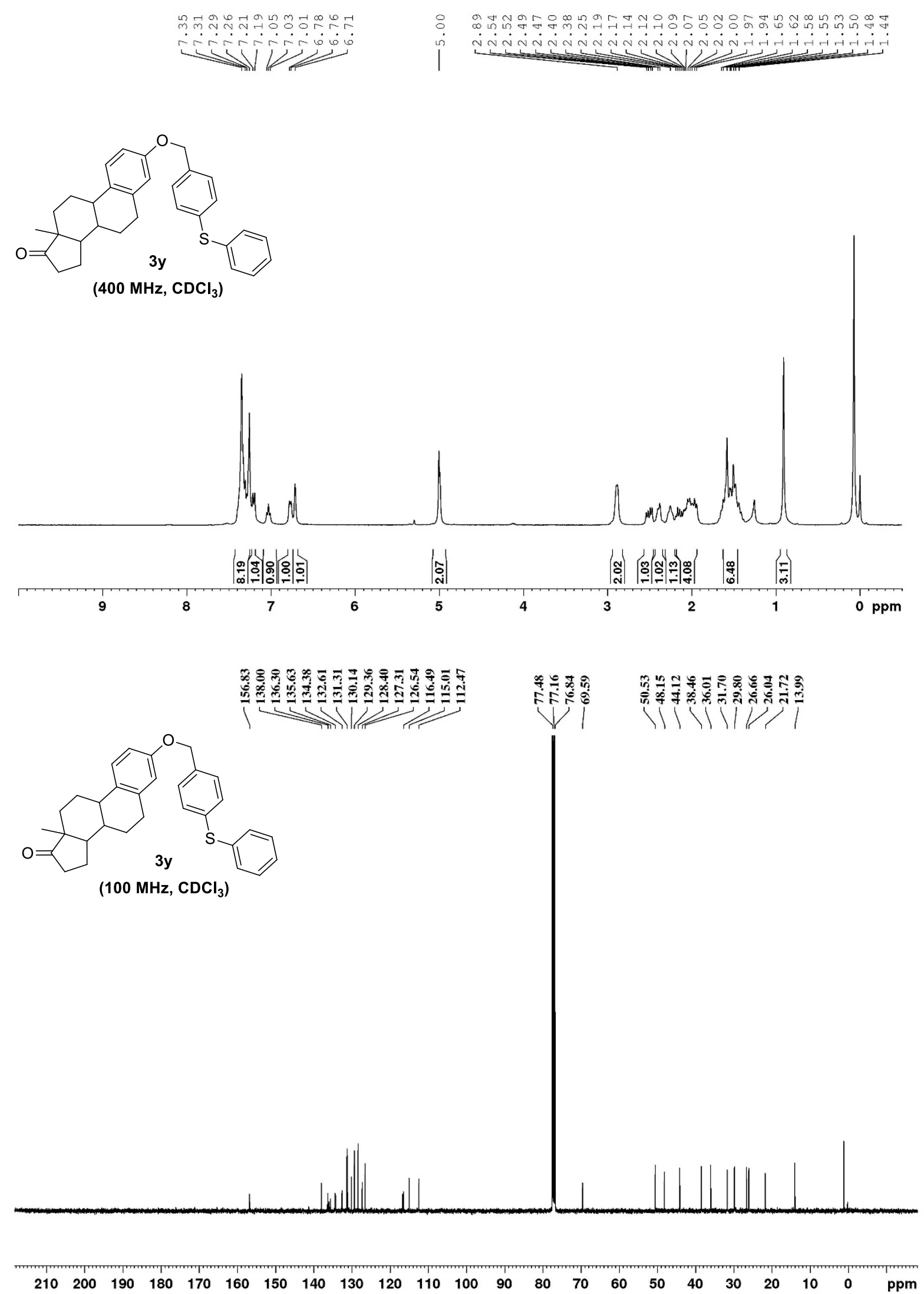

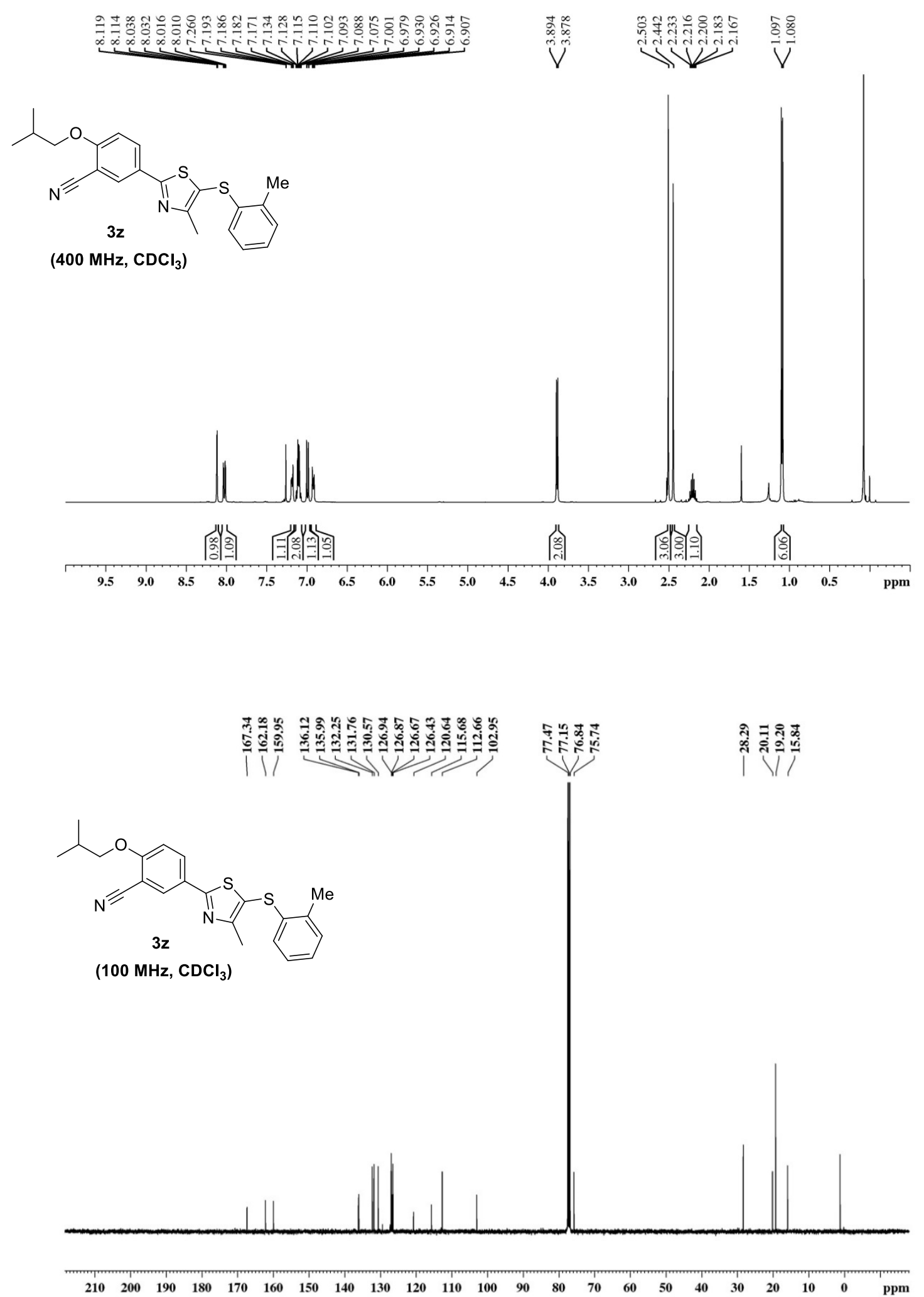

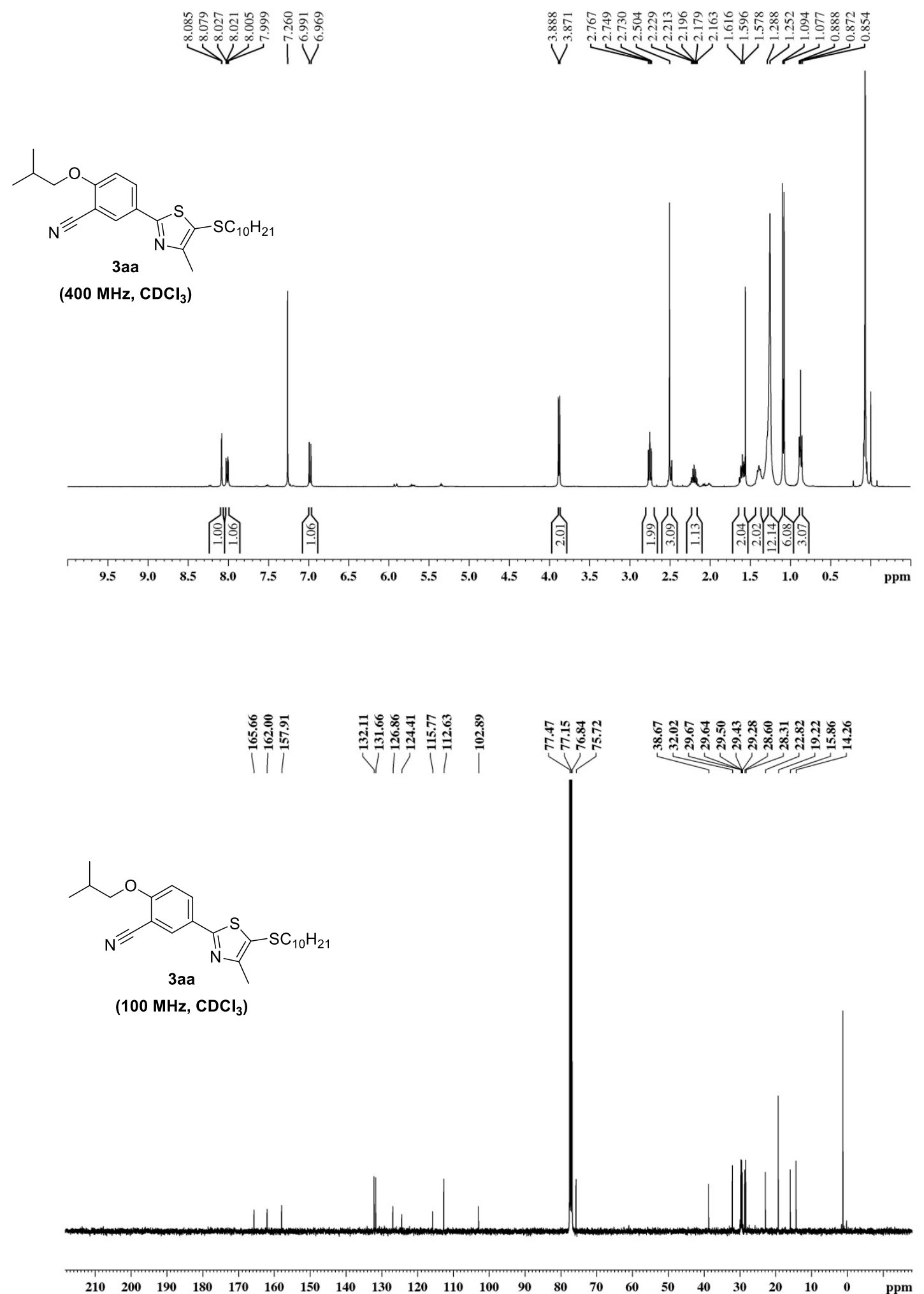